\title{
Prediction of unprecedented biological shifts in the global ocean
}

G. Beaugrand ${ }^{1,2^{*}}$, A. Conversi ${ }^{3}$, A. Atkinson ${ }^{4}$, J. Cloern ${ }^{5}$, S. Chiba ${ }^{6}$, S. Fonda-Umani ${ }^{7}$, R.R. Kirby $^{8}$, C.H. Greene ${ }^{9}$, E. Goberville ${ }^{10}$, S.A. Otto ${ }^{11}$, P.C. Reid ${ }^{2,4}$, L. Stemmann ${ }^{12}$, M. Edwards ${ }^{2,4}$

* Corresponding author: gregory.beaugrand@cnrs.fr

${ }^{1}$ Centre National de la Recherche Scientifique, Laboratoire d'Océanologie et de Géosciences, UMR 8187 LOG, Station Marine de Wimereux, Université de Lille, Université du Littoral Côte d'Opale, Wimereux France

2 Marine Biological Association, the Continuous Plankton Recorder (CPR) survey, The Laboratory, Citadel Hill, The Hoe, Plymouth PL1 2PB, UK

${ }^{3}$ ISMAR-Marine Sciences Institute in La Spezia, CNR-National Research Council of Italy, Forte Santa Teresa, Loc. Pozzuolo, 19032, Lerici, SP, Italy

${ }^{4}$ Plymouth Marine Laboratory, Prospect Place, The Hoe, Plymouth PL13DH, UK

${ }^{5}$ USGS MS496 345 Middlefield Rd., Menlo Park, CA, 94025, USA

${ }^{6}$ RCGC, JAMSTEC, 3173-25 Showa-machi, Kanazawa-ku, Yokohama, 236-0001 Japan

${ }^{7}$ Department of Life Sciences, University of Trieste, v. Giorgieri, 10, 34127 Trieste, Italy

${ }^{8}$ The Secchi Disk Foundation, Kiln Cottage, Gnaton, Yealmpton, PI8 2HU, UK

${ }^{9}$ Ocean Resources and Ecosystems Program, Cornell University, Ithaca, NY 14853-1504, USA

${ }^{10}$ BOREA, MNHN, Sorbonne Université, Université de Caen Normandie, Université des Antilles, CNRS, IRD, CP53, Paris, France.

${ }^{11}$ Institute of Marine Ecosystem and Fishery Science (IMF), Center for Earth System Research and Sustainability, University of Hamburg, 22767 Hamburg, Germany

${ }^{12}$ Sorbonne Université, Institut de la Mer de Villefranche, CNRS, UMR 7093, Laboratoire d'Océanographie de Villefranche, F-06230, Villefranche/mer, France 


\begin{abstract}
Impermanence is an ecological principle ${ }^{1}$ involving changes that can sometimes occur non-linearly as Abrupt Community Shifts (ACSs) to transform ecosystem states and the goods and services they provide $^{2}$. Here, we present a model based on niche theory ${ }^{3}$ to explain and predict ACSs at the global scale. We test our model using 14 multi-decadal time series of marine metazoans from zooplankton to fish, spanning all latitudes and the shelf to the open ocean. Predicted and observed fluctuations correspond, with both identifying ACSs at the end of the $1980 \mathrm{~s}^{4-7}$ and $1990 \mathrm{~s}^{5,8}$. We show that these ACSs coincide with changes in climate that alter local thermal regimes, which in turn interact with the thermal niche of species to trigger long-term and sometimes abrupt shifts at the community level. A large-scale ACS is predicted after 2014 - unprecedented in magnitude and extent - coinciding with a strong El Niño event and major shifts in Northern Hemisphere climate. Our results underline the sensitivity of the Arctic Ocean, where unprecedented melting may reorganize biological communities ${ }^{5,9}$ and suggest an increase in the size and consequences of ACS events in a warming world.
\end{abstract}

\title{
Main text
}

The processes that cause long-term changes and Abrupt Community Shifts (ACSs) in ecosystems are poorly understood despite decades of research ${ }^{2,4,10-12}$. We define an ACS as a stepwise shift in community structure ${ }^{12}$, a definition that does not necessarily imply the existence of stable states ${ }^{2,10}$, which are rarely observed in pelagic ecosystems ${ }^{10,12-14}$. Such ACSs correspond to rapid and major alterations in species composition ${ }^{15}$, which alter biodiversity with consequences for ecosystem services. Here, we apply a framework based on the MacroEcological Theory on the Arrangement of Life (METAL; Methods and Supplementary Table 1 for a list of acronyms) ${ }^{12,16-18}$. METAL integrates key ecological concepts (e.g. the ecological niche sensu Hutchinson) into a unique and coherent scheme that unifies space and time patterns at both species and community levels and enables quantifiable predictions. METAL has been used to explain responses of species and communities to climate change and the large-scale arrangement of biodiversity ${ }^{12,16-18}$.

We applied the METAL-based model to investigate long-term community changes and ACSs in pelagic marine metazoans in the global ocean from 1960 to 2015. In each geographical cell of a gridded ocean, we built pseudo-communities from a pool of pseudo-species, i.e. simulated species characterised by unique thermal niches ranging from stenotherms (species with narrow temperature tolerance) to eurytherms (adapted to extreme temperature variations) and from psychrophiles (adapted to cold temperatures) to thermophiles (adapted to warm temperatures) ${ }^{12}$. Pseudo-communities were built 
from pseudo-species adapted to temperature fluctuations in a given region. We therefore focused on climate-induced changes that originate from fluctuations in the thermal regime and not from shifts induced by other environmental parameters ${ }^{19}$ (e.g. nutrients, salinity, oxygen) or anthropogenic pressures (e.g. fishing, eutrophication and pollution) ${ }^{2}$. It is now a key principle in ecology that thermal tolerance is species-specific ${ }^{3}$ and the strong influence of temperature on species through their thermal niche has been observed for a variety of ecosystems and taxonomic groups ${ }^{17,20,21}$. The novelty in this present analysis is our conceptual incorporation of the niche into a model that allows us to explore the influence of temperature at the community level in the context of climate-induced changes, including ACSs.

We first tested if our model's predictions of long-term changes in pseudo-communities agreed with independent observations from 14 multi-decadal series (Supplementary Figure 1; Supplementary Tables 2-3). These records included measurements of zooplankton (11 ecosystems), decapods (1 ecosystem), and/or fish (4 ecosystems) spanning tropical (Hawaii) to polar regions (Southern Ocean), coastal (e.g. Adriatic and San Francisco Bay) to deep/open oceans (Pacific, Atlantic and Southern Oceans) and seas (Adriatic, Ligurian, North and Baltic Seas). For each observed community we performed a standardised Principal Components Analysis (PCA) on a matrix of years by biological variables (e.g. species abundance, biomass, or size fraction) and retained the first two Principal Components to investigate their long-term changes (Obs-PC1-PC2s). In each system, 10,000 PCAs were also performed on 10,000 pseudo-communities, each resulting from different simulated pseudospecies associations. From these, we retained 10,000 pairs of predicted changes (Pred-PC1-PC2s) ${ }^{12}$. We provide an illustrative case-example of the skill of our model for the North Sea (Figure 1). Here, the first obs-PC shows a pronounced change at the end of the 1980s for $43 \%$ of taxa, and our simulated first PC also exhibits the same pattern for $71 \%$ of the pseudo-species; both PCs were highly correlated (Figure 1a-b,e-f; $r=0.83, p=0.01, n=50$ ).

When all 14 ecoregions are considered (Fig. 2a-n), 89\% (25) of all first two observed eigenvalues are significant (Supplementary Table 4). The observed and predicted community changes are highly correlated for every ecoregion, except for the highly dynamic and heterogeneous ${ }^{22}$ Western Pacific Transition Zone where only the two late-1980s and -1990s substantial shifts were predicted (Figure 2; Supplementary Note 1 and Table 5). Note that long-term changes shown in the figure are the examples showing the highest correlations between an observed and a predicted PC. As it might be expected, some observed PCs were weakly correlated with predicted PCs (Supplementary Table 5), which could be because i) some species may not react to temperature when their thermal optimum coincides with the mean local thermal regime ${ }^{12,23}$, or ii) some species may be more sensitive to other forcing (e.g. 
anthropogenic pressure, other ecological factors, biotic interactions or local complex circulation patterns) $)^{5,19,22,24}$. Such differences in response may explain why the climatic signal identified by the PCAs was sometimes associated with PC1 and at other times with PC2. Therefore, to consider the full complexity of the signal, we used the first 2 predicted PCs (mean Pred-PC1-2, averaged over 10,000 simulated PCs; Figure 2a-n) and applied a regression (Methods) that explained $50.4 \%$ $(r=0.71, p<0.01, n=567$,Fig. 2o) of the total variance of all observed long-term changes in the 14 ecoregions (red Obs-PCs; Figure 2a-n). Since predicted communities are independent of the observed communities, correlations of observed and predicted PCs are higher than we expected. This suggests that our framework captures the main drivers of changing communities.

To verify that these results were not due to chance, we also examined correlations between observations and null models generated for each system and composed of random time series both with and without autocorrelation (Methods). Simulated Pred-PC1-PC2s from these null models exhibited a pronounced variability in each system, far exceeding that originating from METAL (Supplementary Figure 2 versus Figure 2a-n). All mean correlations were smaller than those expected from METAL, with the exception of the HOT time series. When all PCs were combined together (Fig. 2o-p), the correlations based on METAL were substantially higher than could be explained by chance (Figure 2p). The small range in METAL/observation correlations (Fig. 2p, red bar) in comparison to null models (Fig. 2p, green and blue) indicates that results are stable, regardless of the selected pseudospecies. This suggests that inclusion of all species might not be necessary to assess a community's state. This is fortuitous because most monitoring programmes sample only a small part of a community (Supplementary Table 3).

Next, we tested the capability of our framework to reveal large-scale community changes, including ACSs, by combining results from all 14 ecoregions (Figure 3). To extract the overall biological variability, we performed 'global' PCAs on the first two Pred-PCs extracted from (i) simulated (METAL and the null model based on autocorrelated time series) and (ii) observed communities for the 14 systems (14 systems $\times 2$ PCs $=28$ variables for each PCA). Therefore, we used all of the first two observed and predicted PCs for this analysis, which represents the full set of interactions within the observed and predicted PCs (Supplementary Table 6). We chose the period 1960-2007 because it had less than 50\% missing data per year in each time series (Supplementary Figure 3). We performed this procedure in two ways: (i) a single 'global' PCA based on the average of 10,000 PC1-PC2s for each system to calculate the correlation between observations and predictions from METAL, and (ii) 10,000 'global' PCAs to compare the variability of long-term changes from METAL and the null model. The first global PC originating from observed communities was highly correlated $(r=0.87)$ with the first global PC derived 
from the mean of 10,000 theoretical communities (Figure 3a). Predicted and observed PC2s and PC3s were also significantly correlated, although at a lower level (Figure 3b-c). METAL predictions had smaller variability than predictions based on the null model (Figure 3a-c, green versus blue curves).

We verified the representativity of these time series with respect to the global ocean. While our 14 initial sites or ecoregions span a range of water depths from 31 to $5492 \mathrm{~m}$, many are close to the coastline and so our results could be biased towards shallow marine environments (Supplementary Table 3). To address this possibility, we analysed observations in 5 additional regions of the North Atlantic where we had spatially and temporally consistent data from nearshore and offshore and from shallow to deep waters (Supplementary Note 1). This analysis confirmed the validity of our framework in open oceanic domains (Supplementary Note 1) even if no data were available to us from the centre of oceanic basins nor from the South Pacific, South Atlantic or Indian Oceans (Supplementary Note 1).

We then tested METAL predictions of abrupt shifts. Using pooled data from the 14 initial sites, we identified ACSs using an Abrupt Shift Detection (ASD) algorithm on 10,000 global PC1-PC3s (Methods). The algorithm detected a significant shift circa 1987 in our North Sea example for both predicted and observed first PCs (Figure 1c-d). For global PCs, the first two observed and predicted PCs showed significant ACSs at the end of the 1980s and 1990s respectively (Figure 3d-e). Predicted ACSs (blue curves) occurred one year before observed ACSs (red curves), possibly reflecting inertia related to species' life cycles ${ }^{16}$. This analysis shows that the most frequent (but not necessarily the most intense) shift in the ecoregions coincided with the well-documented events of the late 1980s (Figure 3a,d) ${ }^{4,6,7}$. Although they were not significant, we detected acceleration phases on both third predicted and observed PCs (Figure 3c,f). The well documented 1976/77 ACS ${ }^{15}$ was not significant when all systems were considered (Fig. 3), probably because our observations did not include many areas where this shift occurred (Figure 2k, Supplementary Note 1). No significant trends were observed in global PCs based on autocorrelated time series (Figure $3 d-f$, green curves). These results suggest that our model can predict a substantial part of long-term community change, including ACSs.

We then used our ASD algorithm to predict ACSs in space and time by applying it to the whole ocean for the period 1960-2015 and covering areas and years not monitored. For this analysis, we did not use any PCA but applied our algorithm to pseudo-species and retained only ACSs when they involved half or more pseudo-species for a given location and year. Our analysis suggests that ACSs may occur every year, but only in a limited part of the ocean ( $2.8 \%)$, involving on average an area of $\sim 10$ million $\mathrm{km}^{2}$ of ocean per year (Figure 4a and Supplementary Figure 4). Some periods had geographically limited ACSs (e.g. 0.89 million $\mathrm{km}^{2}$ for 1984-1987) whereas others showed more extensive shifts (e.g. 50.5 
million $\mathrm{km}^{2}$ for 2012-2015). Widespread predicted ACSs were always observed after El Niño events (e.g. weak El Niño episode of 1977-1978 and very strong episodes of 1997-1998 and 2015-2016) but not all El Niño events led to widespread ACS predictions (e.g. very strong episodes of 1982-1983; Figure 4a). The late-1980s ACS, so frequently found in areas where monitoring took place $e^{4-6,8,11,12}$, was not predicted on a global scale. Despite similar strength in the two strong El Niño events 1997-1998 and 2015-2016, the spatial extent of the predicted ACSs was very different, the recent one being more widespread (50 million $\mathrm{km}^{2}$ circa 2014 versus 29 million $\mathrm{km}^{2}$ circa 1999). The mean magnitude of ACSs increased substantially after the mid-2000s with a peak circa 2012 (Figure 4b). When both spatial extent (number of geographical cells) and magnitude were combined, an unprecedented shift (5 and 3 times the average extent and magnitude, respectively) occurred after 2010 with a maximum 2014 (Figure 4c). Our null model (Figure 3d-f) showed that such an ACS at the end of the time series is unlikely to be an artifact as is sometimes reported with other techniques ${ }^{4}$ (Methods).

The next question was whether the predicted ACSs could be associated to climate. We investigated these relationships using six climate parameters measured at a global scale: annual Sea Level Pressure (SLP), meridional and zonal winds, wind intensity, cloudiness, and Sea Surface Temperature (SST) (Methods). Using maps of ACSs and climatic shifts calculated for each year (Supplementary Figures 45), we found significant correlations (Supplementary Table 7) between the spatial extent of both predicted and observed ACSs from 1960 to 2015 with annual SLP ( $\left.r=0.69, p_{A C F}<0.01, n=53\right)$, atmospheric circulation (wind intensity and direction) variables $\left(r=0.54-0.57, p_{A C F}<0.01, n=53\right)$ and, as expected, with annual SST $\left(r=0.97, p_{A C F}<0.01, n=53\right)$. No significant correlation was found with cloudiness. These results identify a strong link between the spatial extent of predicted ACSs and shifts in atmospheric circulation and SST.

We subsequently calculated predicted shifts on a global scale, focusing on 5 time periods: 1975-1979, 1985-1989 and 1995-1999 because these include previously documented ACS ${ }^{4-8,11,15}, 2005-2009$ as an example of a relatively stable period; and 2010-2014 because of its exceptional nature (Figure 5). The predicted 2014-2015 ACS, clearly evident by visual inspection (Supplementary Figure 4), is much more intense and widespread than previous shifts (Figures 4-5), encompassing meteo-oceanic warm anomalies in the Northwest Atlantic, Northeast Pacific Ocean and many areas of the Arctic Ocean and the central North-Atlantic cold blob ${ }^{9,25-27}$ (Figure 5). Although changes in the North Atlantic and Pacific Oceans resulted in part from changes in atmospheric and oceanic circulation and its influence on regional thermal regimes, changes in the Arctic resulted mainly from abrupt shifts in annual SST (Figure $5 e, j$, Supplementary Figure 6). 
Our framework provides a theoretical explanation for long-term biological changes and ACSs. Each species responds individually, depending upon the interaction between its thermal niche and fluctuations in the thermal environment ${ }^{12}$, with cumulative responses leading to ecosystem shifts. The close correspondence between shifts in predicted and observed communities supports our framework and provides a useful basis for predicting climate/temperature-induced ACS at the community scale. However, large unexpected events such as the collapse or explosive growth of some populations (black-swan events ${ }^{28}$ ) may not be predicted by our approach.

Our ability to resolve the spatial extent of oceanic community shifts is severely constrained by a paucity and unrepresentative coverage of observations. Most marine communities, in particular marine metazoans, are hidden from earth observation tools and adequate monitoring coverage for the entire ocean is logistically unlikely. Our framework could therefore be meshed with existing monitoring programmes to provide a macroscopic tool for identifying regions likely to develop ACSs and to help anticipate biological perturbations that could affect production of ecosystem goods and services ${ }^{27}$. For example, our framework has predicted an ACS of unprecedented scale in 2014-2015 that may have substantial ecological consequences ${ }^{25,27}$ across the Northern Hemisphere, including in the Arctic where current changes such as sea-ice melting are accelerating ${ }^{9}$. Finally, our study alerts us to the potential for a growing size and consequence of future ACS events as the world warms in response to rapidly increasing concentrations of atmospheric greenhouse gases. Even though it will remain difficult to predict ACSs, both because of model uncertainties and the fact that some event types will remain unpredictable $\mathrm{e}^{29,30}$, the ability to forecast putative ACS events is an important development in our understanding of climate change biology.

\section{Acknowledgements}

This work was supported by the 'Centre National de la Recherche Scientifique' (CNRS), the Research Programme CPER CLIMIBIO (Nord-Pas de Calais), the regional programme INDICOP (Pas-de-Calais) and the ANR project TROPHIK. The authors also thank the French Ministère de l'Enseignement Supérieur et de la Recherche, the Hauts de France Region and the European Funds for Regional Economical Development for their financial support to this project. We are indebted to Philippe Notez for his help in computer engineering. AA and the Antarctic dataset was supported by the World Wildlife Fund and NERC and Department for Environment, Food and Rural affairs (DEFRA) grant NE/L 003279/1 (Marine Ecosystems Research Programme). PCR was also funded by NERC. We thank the anonymous reviewers for their competent and accurate comments that improved the original version of the manuscript. 


\section{Author contributions}

G.B. conceived the study; G.B., A.C., A.A., E.G., J.C., S.C. and all co-authors compiled the data and G.B. analysed the data. G.B. wrote the (initial draft) paper. G.B., A.C., A.A., P.C.R., E.G., J.C., R.R.K., S.O., S.C., M.E. and all other co-authors discussed the results and contributed to the paper writing.

\section{Additional information}

The authors declare no competing financial interests. Supplementary information accompanies this paper at www.nature.com. Reprints and permissions information are available online at www.nature.com/reprints.

To whom correspondence should be addressed: gregory.beaugrand@cnrs.fr

\section{Data availability}

The authors declare that data supporting the findings of this study are available from the corresponding author upon request.

\section{Literature cited}

1 Boero, F. et al. From biodiversity and ecosystem functioning to the roots of ecological complexity. Ecological Complexity 1, 101-109 (2004).

2 Scheffer, M. Critical transitions in nature and society. (Princeton University Press, 2009).

3 Hutchinson, G. E. An introduction to population ecology. (Yale University Press, 1978).

4 Reid, P. C. et al. Global impacts of the 1980s regime shift. Global Change Biology 22, 682-703, doi:10.1111/gcb.13106 (2016).

5 Greene, C. H., Pershing, A. J., Cronin, T. M. \& Ceci, N. Arctic climate change and its impacts on the ecology of the North Atlantic. Ecology 89, S24-S38 (2008).

6 Conversi, A. et al. The Mediterranean Sea regime shift at the end of the 1980s, and intriguing parallelisms with other European basins. PLOS one 5, 1-15 (2010).

7 Beaugrand, G. et al. Synchronous marine pelagic regime shifts in the Northern Hemisphere. Philosophical Tansactions of the Royal Society B: Biological Sciences 370, 20130272, doi:10.1098/rstb.2013.0272 (2015).

8 Luczak, C., Beaugrand, G., Jaffré, M. \& Lenoir, S. Climate change impact on Balearic Shearwater through a trophic cascade. Biology Letters 7, 702-705 (2011). 
9 Arctic Council. Arctic Resilience Report., (Stockholm Environment Institute and Stockholm Resilience Centre, Stockholm, 2016).

10 Conversi, A. et al. A holistic view of marine regime shifts. Philosophical Transations of the Royal Society B 370, 20130279, doi:10.1098/rstb.2013.0279 (2015).

11 Möllmann, C. \& Diekmann, R. Marine ecosystem regime shifts induced by climate and overfishing: A review for the Northern hemisphere. Advances in Ecological Research 47, 303-347 (2012).

12 Beaugrand, G. Theoretical basis for predicting climate-induced abrupt shifts in the oceans. Philosophical Tansactions of the Royal Society B: Biological Sciences 37020130264 , doi:10.1098/rstb.2013.0264 (2015).

13 Mac Nally, R., Albano, C. \& Fleishman, E. A scrutiny of the evidence for pressure-induced state shifts in estuarine and nearshore ecosystems. Austral Ecology 39, 898-906 (2014).

14 Di Lorenzo, E. \& Ohman, M. D. A double-integration hypothesis to explain ocean ecosystem response to climate forcing. Proceedings of the National Academy of Sciences of the United States of America 110, 2496-2499, doi:10.1073/pnas.1218022110 (2013).

15 Hare, S. R. \& Mantua, N. J. Empirical evidence for North Pacific regime shifts in 1977 and 1989. Progress in Oceanography 47, 103-145 (2000).

16 Beaugrand, G., Goberville, E., Luczak, C. \& Kirby, R. R. Marine biological shifts and climate. Proceedings of the Royal Society B: Biological Sciences 281, 20133350, doi:10.1098/rspb.2013.3350 (2014).

17 Beaugrand, G., Edwards, M., Raybaud, V., Goberville, E. \& Kirby, R. R. Future vulnerability of marine biodiversity compared with contemporary and past changes. Nature Climate Change 5, 695701, doi:10.1038/NCLIMATE2650 (2015).

18 Beaugrand, G. Marine biodiversity, climatic variability and global change., (Routledge, 2015).

19 Cloern, J. E. \& Jassby, A. D. Drivers of change in estuarine-coastal ecosystems: discoveries from four decades of study in San Francisco Bay. Reviews of Geophysics 50, rg4001, doi:10.1029/2012RG000397 (2012).

20 Sunday, J. M., Bates, A. E. \& Dulvy, N. K. Global analysis of thermal tolerance and latitude in ecthoterms. Proceedings of the Royal Society B 278, 1823-1830 (2011).

21 Stuart-Smith, R. D., Edgar, G. J., Barrett, N. S., Kininmonth, S. J. \& Bates, A. E. Thermal biases and vulnerability to warming in the world's marine fauna. Nature 528, 88-92 (2015).

22 Di Lorenzo, E. et al. Synthesis of Pacific Ocean climate and ecosystems dynamics. Oceanography 26, 68-81 (2014).

23 Beaugrand, G. \& Kirby, R. R. Quasi-deterministic responses of marine species to climate change. Climate Research 69, 117-128, doi:10.3354/cr01398 (2016). 
24 Mollmann, C. et al. Reorganization of a large marine ecosystem due to atmospheric and anthropogenic pressure: a discontinuous regime shift in the Central Baltic Sea. . Global Change Biology 15, 1377-1393 (2009).

25 Bond, N. A., Cronin, M. F., Freeland, H. \& Mantua, N. Causes and impacts of the 2014 warm anomaly in the NE Pacific. Geophysical Research Letters 42, 3414-3420, doi:10.1002/2015/GL063306 (2015).

26 Duchez, A. et al. Drivers of exceptionally cold North Atlantic Ocean temperatures and their link to the 2015 European heat wave. Environmental Research Letters 11, 074004, doi:10.1088/17489326/11/7/074004 (2016).

27 Greene, C. H. North America's iconic marine species at risk due to unprecedented ocean warming. Oceanography 29, 14-17 (2016).

28 Aarssen, L. W. High productivity in grassland ecosystems: effected by species diversity or productive species? Oikos 80, 183-184 (1997).

29 Boettiger, C. \& Hastings, A. Early warning signals and the prosecutor's fallacy. Proceedings of the Royal Society B 279, 4734-4739 (2012).

30 Schindler, D. \& Hillborn, R. Prediction, precaution and policy under climate change. Science 347, 953-954 (2015).

\section{Figure legends}

Figure 1 | Long-term biological changes and abrupt community shifts (ACSs) for both the observed community and a simulated pseudo-community in the North Sea. a-b. Long-term changes of the first principal components (PCs) and in biological variables related to them (i.e. absolute values of the normalized eigenvector $\geq 0.6$; blue): (a) observed species and (b) simulated pseudo-species. The first $\mathrm{PC}$, reflecting major changes in community structure, is in black for observed taxa $(\mathbf{a}, \mathbf{e})$ and red $(\mathbf{b}, \mathbf{e})$ for simulated pseudo-species. Taxa related to the observed first PC included Calanus finmarchicus (negative relationship, -), C. helgolandicus (positive relationship, +), Candacia armata (+), Centropages typicus (+), Corycaeus spp. (+), and Oithona spp. (-). Ten pseudo-species were related to the simulated first PC. The grey band shows the timing of the ACS revealed in panels c-d. c-d. Detection of ACSs for the first PC based on (c) the observed community and (d) the simulated pseudo-community. The dashed red horizontal line indicates the threshold of 3 used throughout this study. e-f. Measured (black) and simulated (red) first PCs (e) and their relationships (f). This ecosystem, and most biological systems considered in this study (except HOT, Southern Ocean and San Francisco Bay; see Methods) were also analysed in detail by Beaugrand ${ }^{12}$ and Beaugrand and colleagues ${ }^{7}$, respectively. 
Figure 2 | Predicted (grey) and observed (red) long-term community changes for 14 systems. Principal components were standardised between -1 and 1. Pred-PC: 10,000 Principal Components (grey) based on 10,000 simulated communities. Obs-PC: Principal Component based on observed communities (red). a. North Sea (Pred-PC1s and Obs-PC1), b. Baltic Sea (Pred-PC1s and Obs-PC1), c. Adriatic Sea (Pred-PC1s and Obs-PC1), d. Ligurian Sea (Pred-PC2s and Obs-PC1), e. Northwest Atlantic, southern area (Pred-PC1s and Obs-PC2), f. Northwest Atlantic, northern area (Pred-PC1s and Obs-PC1), g. West Pacific Transition zone (Pred-PC1s and Obs-PC1), h. Oyashio (Pred-PC1s and Obs-PC1), i. CALCOFI (Pred-PC1s and Obs-PC1), j. San Francisco Bay (Pred-PC1s and Obs-PC1), k. East Pacific region (Pred-PC1s and Obs-PC1), I. West Pacific region (Pred-PC1s and Obs-PC2), m. HOT (Pred-PC1s and ObsPC2), n. Antarctic Peninsula area (Pred-PC1s and Obs-PC2). a-n. $r_{m}$ are the mean linear correlations between the mean of 10,000 predicted and the observed community PC. Biological variables considered at each site are indicated by $\mathbf{D}$ (benthic decapods), $\mathbf{Z}$ (zooplankton), and $\mathbf{F}$ (Fish). Biological variables are indicated in the Methods. $\mathrm{x} \mid \mathrm{y}: \mathrm{x}$ is the number of pseudo-species used in METAL and $\mathrm{y}$ is the number of time periods ( 1 indicates the annual value). The total number of variables used in METAL model is the product of $x$ and $y$. For California Current (total zooplankton biomass) and Hawaii (zooplankton size fractions), we chose arbitrarily pseudo-communities composed of 30 pseudospecies. o. Long-term community shifts predicted from a regression on principal components, using the first two Pred-PCs from the 14 systems (averaged from the 10,000 first 2 PCs) and observed community shifts as in panels a-n. p. Histograms of the 10,000 correlations between selected Obs-PCs and predictions based on the first 2 Pred-PCs from (i) the null model based on random time series (grey), (ii) randomly generated time series with an order-1 autocorrelation $\geq 0.5$ (blue), and (iii) the METAL theory (red).

Figure 3 | Comparisons of observed (red) and predicted (blue and green; 10,000 simulations) community shifts, all ecoregions combined. Model predictions are in blue and predictions from a null model with autocorrelation in green. (a) First PC (30.72\% of the total variance): predicted and observed community changes. (b) Second PC (21.46\%): predicted and measured community changes. (c) Third PC (15.47\%): predicted and observed community changes. Index of abruptness of predicted and observed community changes: (d) First PC. (e) Second PC. (f) Third PC. Correlation (r), probability of significance without $(p)$ and with ( $p_{A C F}$ ) correction for temporal autocorrelation, and degree of freedom ( $n$ ) are indicated in panels $\mathbf{a}, \mathbf{b}$ and $\mathbf{c}$ and correspond to the correlation calculated between observations and METAL predictions when all local PCs are averaged. In d-f, the wide blue (METAL) and green (null model) curves correspond to the medians of 10,000 simulations and the lower and upper part, are the $5^{\text {th }}$ and $95^{\text {th }}$ percentiles, respectively. The first three axes of the PCA performed on observed data were significant using a statistical test based on a broken-stick distribution (Methods). 
Figure 4 | Predicted long-term variation of Abrupt Community Shifts (ACSs) in the global ocean. (a) spatial extent, (b) magnitude and (c) spatial extent and magnitude of ACS. Curves in red are order-1 moving average of predicted values (blue bars) (Methods). Thin-dashed, thin-solid and thick red arrows identify weak, moderate, and strong El Niño events, ' $E$ ' = super El-Niño events. Thin and thick blue arrows identify moderate and strong La Niña events.

Figure 5 | Predicted Abrupt Community Shifts (ACSs; a-e) and climatic shifts (f-j) during the period 1960-2015 with a focus on the years 1975-1979 ( $\mathrm{a}$ and f), 1985-1989 (b and g), 1995-1999 (c and h), 2005-2009 ( $d$ and i) and 2010-2014 (e and j). Colour bars show the percentage of individual time series having a significant shift (threshold $>3$ ). For ACSs, 50\% means that half the pseudo-species exhibited a significant shift for a given pseudo-community. For climatic shifts, $50 \%$ means that half the climate parameters ( 3 of 6 parameters) had a significant shift. White areas are regions with no shift. When the percentage of shifts is $>0$, the percentage is indicated by a colour: yellow and red for low and high percentage, respectively. The six climatic parameters are: annual Sea Level Pressure (SLP), meridional wind, zonal wind, wind intensity, cloudiness and annual sea surface temperature (SST). The spatial extent of ACSs increases when the climatic shifts are more widespread. Individual maps of all predicted ACSs and observed climatic shifts are displayed in Supplementary Figures 4-5. Black arrow: direction and intensity of mean annual wind (1960-2015). Black line: isobar based on mean annual SLP (19602015). 


\section{Methods section}

\section{Materials}

\section{Sea surface Temperature}

Annual SSTs originated from the dataset ERSST_v3 (1960-2015). The dataset is derived from a reanalysis based on the most recently available International Comprehensive OceanAtmosphere Data Set (ICOADS). Improved statistical methods have been applied to produce a stable monthly reconstruction, on a $2^{\circ} \times 2^{\circ}$ spatial grid, based on sparse data ${ }^{31}$. Data were interpolated on a global grid of $1^{\circ}$ latitude $\times 1^{\circ}$ longitude.

\section{Sea Level Pressure, wind and cloudiness}

Sea Level Pressure (SLP), cloudiness and both the meridional (V) and the zonal (U) components of the wind were extracted from the National Center for Environmental Prediction/National Center for Atmospheric Research (NCEP/NCAR) Reanalysis project ${ }^{32}$. NCEP uses a climate model that is initialised with observations originating from a variety of sources (e.g. ships, planes and satellite observations). The spatial grid $\left(2.5^{\circ}\right.$ latitude $\times 2.5^{\circ}$ longitude) of annual average SLP and $U$ and $V$ wind data were constructed for the period 1960-2015. Wind intensity was calculated from $U$ and $V$ wind. Data were interpolated on a global grid of $1^{\circ}$ latitude $\times 1^{\circ}$ longitude for the period 1960-2015.

\section{Observed biological data}

We used a summary of long-term community shifts (i.e. the first 2 principal components after applying a Principal Components Analysis, PCA) in 14 regions located in three oceans (the Atlantic, the Pacific and the Southern Oceans) and four Longhurst biomes ${ }^{33}$ (Polar, Westerlies, Trade-Winds, and Coastal biomes). The first 11 regions have been analysed by standardised PCA in Beaugrand and colleagues ${ }^{7}$, the $12^{\text {th }}$ has been analysed in Cloern and colleagues using the same technique ${ }^{34}$ and both the $13^{\text {th }}$ and $14^{\text {th }}$ time series were added to the present study, following the same procedure. Supplementary Note 2 summarized the main characteristics of the 14 selected regions (see also Supplementary Figure 1 and Supplementary Table 2 for more details). 


\section{Models and numerical procedures}

\section{Overview of the METAL theory}

We applied a framework based on the MacroEcological Theory on the Arrangement of Life (METAL) $^{12,16-18,35,36}$, a theory that explains how marine pelagic metazoans are arranged in the sea and how changing environmental conditions alter biological arrangements in space and time at different organisational levels (e.g. species, community, ecosystem), allowing precise predictions to be tested. METAL proposes that biodiversity is to a large extent influenced by climate and the environment. This influence mainly takes place through the interactions between the species ecological niche (sensu Hutchinson ${ }^{37}$ ) and both climatic and environmental changes. This interaction determines in large part the arrangement of life in the oceans at different organisational levels from the species to the ecosystem level and from small to large ecosystems ${ }^{18}$. More information on the METAL theory is available in Supplementary Note 3.

\section{Generation of pseudo-species and pseudo-communities}

To construct long-term changes in pseudo-community in each geographical cell of the global ocean, we used here the model described in Beaugrand ${ }^{12}$ and Beaugrand and colleagues ${ }^{17}$; this model only uses one environmental parameter: temperature. We create pseudo-species, each having a unique Gaussian thermal niche with distinct degrees of eurythermy and thermophily ${ }^{16,17,38}$ (Supplementary Figure 7, step 1 ). The response curve of the abundance $E$ of a pseudo-species $s$ in a given site $i$ and time $j$ to change in SSTs was modelled by the following function ${ }^{17,39}$ :

$$
E_{i, j, s}=c_{s} e^{-\left(\frac{\left(x_{i, j}-u_{s}\right)^{2}}{2 t_{s}^{2}}\right)}
$$

With $E_{i, j, s}$ the expected abundance of a pseudo-species $s$ at location $i$ and time $j$; $c_{s}$ the maximum value of abundance for species $s$ fixed to one; $x_{i, j}$ the value of SST at location $i$ and time $j ; u_{s}$ the thermal optimum and $t_{s}$ the thermal amplitude for species $s$. The thermal tolerance is an estimation of the breadth (or thermal amplitude) of the species thermal niche ${ }^{39}$.

A large number of pseudo-species was created with $u_{s}$ varying between $-1.8^{\circ} \mathrm{C}$ and $40^{\circ} \mathrm{C}$ by $0.1^{\circ} \mathrm{C}$ increments and $t_{s}$ varying between $1.1^{\circ} \mathrm{C}$ and $10^{\circ} \mathrm{C}$ by increments of $0.05^{\circ} \mathrm{C}$. This represented a total of 39,218 potential species. However, to consider niche vacancy ${ }^{38}$, we randomly selected half of this number ${ }^{17}$. At the end of the procedure, the global pool of pseudo-species was equal to 19,609; pseudo-species were randomly chosen to create local pseudo-communities (Supplementary Figure 7, step 1) so long as they could withstand the local temperature (annual SST) regime (Supplementary Figure 7, steps 1-3). 
In each geographical cell, a pseudo-community was composed of a given number of pseudospecies (see the map in Supplementary Figure 7). A similar biodiversity map generated by the procedure can also be seen in Beaugrand and colleagues (their figure 1a) ${ }^{17}$. Each pseudospecies has an index of abundance varying between 0 and 1 (Supplementary Figure 7, step 4). The expected abundance of such pseudo-species was determined by linear interpolation from the pseudo-species' thermal niche and monthly SSTs in a given geographical cell from 1960 to 2015 (Supplementary Figure 7, step 4). The procedure has been evaluated in detail for the North Sea by Beaugrand ${ }^{12}$.

A summary of the state of the community is subsequently made by Principal Components Analysis (PCA), using the first two principal components (Supplementary Figure 7, step 5). More details on this analysis are presented in subsequent sections.

\section{Detection of Abrupt Community Shifts}

Many methods have been proposed ${ }^{40,41}$. Shift detection can be accomplished by the use of the coefficient of variation ${ }^{41}$, the measure of the autocorrelation ${ }^{42}$, or the quantification of the multi-scale variance along time series ${ }^{43}$. Here, we calculated an index of abruptness to identify in a simple way Abrupt Community Shifts (ACSs). Our abrupt shift detection algorithm was a simplified version of the methodology developed in Beaugrand and colleagues ${ }^{43}$. This index was developed because (i) it does not require stable states in contrast to other algorithms, (ii) it identifies a shift by evaluating the variability of the time series and (iii) it can be applied in the intensive research of ACSs on a global scale. For a given time series, we first calculated the order-1-5 difference of a time series where observations were standardised between 0 and 1 . Be $\mathbf{Z}$ a time series, $\mathbf{Z}=\left[z_{i}\right]$ was standardised as follows:

$$
x_{i}=\frac{z_{i}-\min (z)}{\max (z)-\min (z)}
$$

The amplitude vector $A^{p}=\left[a^{p_{i}}\right]$ was subsequently calculated as the first $p$ difference between a value of $X=\left[x_{i}\right]$ at year $t+p$ and year $t$, with $1 \leq p \leq 5$ :

$$
a^{p}=\left|x_{t+p}-x_{t}\right|
$$

After transformation, the time series has a length of $n-p$. This transformation enables the time series to become stationary (i.e. constant mean, variance and autocorrelation structure), an important assumption for many statistical tests. The magnitude vector $\mathbf{M}^{\mathbf{p}}=\left[\mathrm{m}^{\mathbf{p}_{i}}\right]$ was subsequently calculated by making the ratio of the amplitude of change a on the average amplitude of the time series:

$$
m_{i}^{p}=\frac{a_{i}^{p}}{\frac{1}{n-p-1} \sum_{j=1, i \neq j}^{n-p} a_{j}^{p}}
$$

The denominator can reach values up to 1 ; therefore, for a constant numerator, the smaller the denominator the higher the values of $\mathbf{M}$. On 100 simulated time series of 10,001 points, 
$95 \%$ of the 10,000 first differences ranged between 2.29 and 2.37 . To be conservative, we selected a detection threshold of 3 . Any values above 3 were considered to be indicative of an abrupt shift. We also used an order-1 symmetrical moving average prior to the application of the abrupt shift detection algorithm to diminish the influence of white noise in biological time series. Test of the procedure can be found in Supplementary Note 4 (see also Supplementary Figures 8-13).

\section{Relationships between observed and predicted long-term community shifts}

To test whether the METAL model was able to reproduce well long-term community shifts (including Abrupt Community Shifts or ACSs), we compared METAL predictions of long-term pseudo-community shifts with observed community shifts in 14 oceanic regions (Supplementary Figure 14). We applied a standardised PCA on a table years $\mathrm{x}$ biological variables and used the first 2 principal components to characterise biological changes in each of the 14 regions for which we had data. For 12 of those regions, the standardised Principal Components Analyses (standardised PCAs) were already performed ${ }^{7}$ and we applied a similar procedure for the two others: (i) HOT station and (ii) Antarctic Peninsula area. Note that the standardised PCA for San Francisco Bay was updated to 2013 using the same procedure ${ }^{34}$. We tested the significance of the first two axes (eigenvalues) by using a broken-stick distribution ${ }^{44}$ (Supplementary Table 7).

We calculated long-term pseudo-community shifts in each of the 14 regions by applying the procedure described in Beaugrand ${ }^{12}$; we produced a pool of pseudo-species that were able to colonise each of the 14 regions so long as they could withstand annual changes in SSTs (Supplementary Figure 7; see the section "Generation of pseudo-species and pseudocommunities"). The generation of the pseudo-species was made using Equation (1) for the period 1960-2015. Many pseudo-species were produced. However, only pseudo-species with an annual relative (i.e. expressed as percentage) abundance $>0.005$ and a presence $>6 \%$ for all years of the time period were kept $^{45}$. As more pseudo-species were generated than observed biological variables involved in the calculations of the summary of each observed community shifts, we chose randomly, for each region, a number of pseudo-species that corresponded to the number of biological variables (Supplementary Table 3 and Supplementary Figure 14). For example in the Pacific Rim, we selected a number of pseudospecies that corresponded to the number of biological variables used to perform the PCA. However, when total zooplankton biomass (CalCOFI) or size fractions (HOT) were used, we arbitrarily chose 30 pseudo-species and also chose two 2-month periods instead of a single 4month period for the Southern Ocean. For CaICOFI and HOT, the selection of this number of pseudo-species did not affect significantly the results above 5 pseudo-species. For the Southern Ocean, the selection of two 2-month periods instead of one 4-month period reduced the variability of the different trajectories but did not strongly affect our conclusions. We repeated the selection of the pseudo-species 10,000 times and recalculated each time the first two principal components on pseudo-species (Supplementary Figure 14). Therefore, 10,000 PCAs were performed for each of the 14 regions. When the number of pseudo-species was high in a given system, variability in the METAL predictions was low.

We subsequently compared the first two observed and predicted Principal Components (PCS) of the 14 regions; Obs-PC for PCs from the PCA based on observed community and Pred-PC 
for PCs from the PCAs based on pseudo-communities (PC1 and PC2 for first and second principal components, respectively). This comparison was made in 3 different ways (Supplementary Figure 14).

First, we calculated the linear correlation coefficients between each Obs-PC and the average of the 10000 Pred-PCs (Supplementary Table 4); when the average was calculated, probabilities were calculated with and without adjusting the degree of freedom to correct for temporal autocorrelation ${ }^{46}$. Based on the correlation coefficients, we represented the best relationships between one of the two Obs-PCs and Pred-PCs for the 14 systems (Figure 2).

Second, we performed a regression on Principal Components ${ }^{47}$ between the Obs-PC selected in Figure 2a-n and the first two corresponding Pred-PCs (averaged for the 10000 simulations) for the 14 systems. Those analyses were performed because they better integrate the complexity of the temporal signal of predictive PCs and removes any bias related to the selection of a given Pred-PC. A scatterplot was then performed between observed and modelled Obs-PCs of all systems and a linear correlation coefficient was calculated (Figure 20). We also repeated the procedure for every simulation (10000) to examine the variability of the correlations between observed and predicted PCs (Figure 2p, red bars).

Third, we calculated two 'global' standardised PCAs: the first, on the matrix that combined the first 2 Obs-PCs obtained from each PCA applied on observed communities; the second, on the matrix that resulted from the combination of the first 2 Pred-PCs obtained from PCAs applied on simulated pseudo-communities (after averaging the 10,000 simulations). Prior to these analyses, we estimated the number of missing data from 1950 to 2014 to select a time period for which years had less than 50\% of missing data (period 1960-2007; Supplementary Figure 3 ). This threshold of $50 \%$ was chosen to have a maximum of years in the analyses with an alteration towards the beginning and the end of the selected time period as low as possible. We examined the relationships between the first 3 global Obs-PCs and Pred-PCs. We tested the significance of the first three axes by using a broken-stick distribution ${ }^{44}$. Normalised eigenvectors (i.e. correlations between long-term changes in the value of each variable with the first three principal components) are in Supplementary Table 5. This analysis focused on the different long-term and more abrupt patterns that were observed in the 14 systems. We also repeated the procedure for every simulation (10000) to examine the variability of the first 3 predicted global PCs (Figure 3a-c; curves in blue) and its influence on the detection of ACSs (Figure 3d-f; curves in blue). We applied our abrupt shift detection algorithm to identify ACSs in the three global Obs-PCs and Pred-PCs. For this analysis, we used an order-3 weighted difference in all analyses after having applied an order-1 symmetrical moving average (see Equation 4 with $p=3$ ). This procedure was chosen to remove the effects of episodic events and to concentrate on ACSs that may have persistent effects.

\section{Null models}

We designed two null models to examine whether our METAL predictions fit better the observations than those obtained randomly (with and without temporal autocorrelation; Supplementary Figure 14). The first null model generated a number of random time series for each station corresponding to the species richness simulated by METAL, with a length corresponding to the time period covered in each sampling site (Figure 2) and a length 
corresponding to the time period chosen to perform 'global' PCAs (Figure 3). The second null model generated the same number of time series but using random time series with an order1 temporal autocorrelation $\geq 0.5$ as many of our observed and METAL-simulated time series were autocorrelated. We analysed those data using the same procedures applied to analyse METAL-simulated data (see the previous section) and to examine the relationships with observed biological data (Supplementary Figure 14). We only represented expected biological changes based on the null model using randomly generated time series with an order-1 autocorrelation $\geq 0.5$ as the null model based on random time series gave similar results. The average correlation between expected and observed changes was reported for each site (Supplementary Figure 2).

Subsequently, we calculated regression on PCs (the first two PCs for each system) using the same procedure as above for every expected Pred-PCs (therefore 10000 times). We performed this analysis for both random time series and randomly generated time series with an autocorrelation $\geq 0.5$. A histogram of all correlations (random time series and randomly generated time series) was performed and compared with correlations based on the METAL model (Figure 2p). Green: correlations between Pred-PCs based on random time series and Obs-PCs; Blue: correlations between Pred-PCs based on randomly generated time series with an order-1 autocorrelation $\geq 0.5$ and Obs-PCs; Red: correlations between Pred-PCs based on the METAL model and Obs-PCs.

We calculated 'global' PCAs based on the 10000 Pred-PCs originating from Pred-PCs based on randomly generated time series with an order-1 autocorrelation $\geq 0.5 ; 10000$ global PCAs were thereby calculated to examine the variability of the first 3 'global' PCs (Figure 3a-c; green). We also applied the Abrupt Shift Detection algorithm on each global PC (1-3 global PCs) and represented the $5^{\text {th }}, 50^{\text {th }}$ (median) and $95^{\text {th }}$ percentiles (Figure $3 \mathrm{~d}-\mathrm{f}$; green).

\section{Predictions of Abrupt Community Shifts}

After testing our theoretical framework and abrupt shift detection algorithm against field observations, we estimated theoretically ACSs from 1960 to 2015 in all areas of the oceans. Here also, only pseudo-species with an annual relative (i.e. expressed as percentage) abundance $>0.005$ and a presence $>6 \%$ for all years of the time period were kept ${ }^{45}$. In each geographical cell of the oceans, we estimated ACSs for all pseudo-species composing a pseudo-community. To estimate ACSs, we used an order-3 weighted difference after having applied an order-1 symmetrical moving average (see Equation 4 with $p=3$ ), as above. Then, we represented for each tested time period from 1960-1963 (mean year 1961.5) to 2012-2015 (mean year 2013.5) the amount of species that exhibit ACSs out of the pseudo-species composing the pseudo-community (Supplementary Figure 4). Data of mean sea level pressure and mean wind circulation were superimposed on maps for the corresponding time period. Because the number of maps was large (see Supplementary Figure 4 for all maps), we chose 5 time periods: (i) 1975-1979, 1985-1989 and 1995-1999 because these include already documented $\mathrm{ACSs}^{4,7,8,15,48}$, (ii) 2005-2009 because it is an example of a relatively calm period and (iii) the last period 2010-2014 (Figure 5). To pool different mean year of shift (e.g. 1976.5 as an average of period 1975-1978) within those time periods (e.g. 1975-1979), we calculated the highest percentage of pseudo-species' shift in each geographical cell (e.g. 1975.5, 1976.5, 1977.5, 1978.5, 1979.5). 


\section{Observations of abrupt climatic shifts}

We applied the same procedure to identify abrupt climatic shifts from 1960 to 2015 . We also applied an order-3 weighted difference after having applied an order-1 symmetrical moving average (see Equation 4 with $p=3$ ). The abrupt shift detection algorithm was performed on annual sea level pressure, meridional (U) and zonal (V) wind, wind intensity, cloudiness and SST. We then added the number of significant shifts observed in each geographical cell from 1960-1963 to 2012-2015 (Supplementary Figure 5). To examine the potential relationships between predicted ACSs and climatic shifts, we applied the same procedure as above for the same time periods: 1975-1979, 1985-1989, 1995-1999, 2005-2009 and 2010-2014 (Figure 4df). Abrupt climatic shifts were mapped for the period 2010-2014 for each climatic variable (2010-2014) in an attempt to understand the exceptional nature of the time period identified by our theoretical framework (Supplementary Figure 6).

\section{Long-term changes in spatial extent and magnitude of abrupt shifts}

For each year, we estimated the spatial extent, the magnitude, and both combined, of ACSs (Figure 5).

\section{Estimation of spatial extent of ACSs}

For each geographical cell that had a percentage of pseudo-species that shifted significantly above $50 \%$ in a given pseudo-community, geographical distances in a geographical cell were calculated as follows ${ }^{49}$ :

$$
d(i, j)=6377.221 \times h_{i, j}
$$

With $d_{i, j}$ being the geographical distance between point $i$ and $j$, the constant the Earth radius and $h_{i, j}$ computed as follows ${ }^{49}$ :

$$
h_{i, j}=\operatorname{arcos}\left(\sin \varphi_{i} \sin \varphi_{j}+\cos \varphi_{i} \cos \varphi_{j} \cos g\right)
$$

With $\varphi_{i}$ the latitude (in radians) at point $\mathrm{i}, \varphi_{\mathrm{j}}$ the latitude (in radians) at point $\mathrm{j}$ and $\mathrm{g}$ the difference in longitude between $i$ and $j$. The area was subsequently calculated by multiplying the zonal and meridional distance of the cell. Finally, we added all areas to obtain the spatial extent concerned by ACSs (Figure 4a). The same type of calculation was applied for each climatic variable that shifted significantly. This procedure allowed us to calculate the correlation (and its probability of significance $p_{A C F}$; ACF means autocorrelation function) between long-term changes in spatial extent of significant ACSs and the spatial extent of climatic shift (threshold of 3 ) for each variable taken individually (Supplementary Table 6).

\section{Estimation of the magnitude of ACSs}

We also estimated the magnitude of ACSs for each year of the time period (1960-2015) by averaging the index of abruptness for each geographical cell for which more than $50 \%$ of pseudo-species shifted significantly (threshold of 3; Figure 4b). 


\section{Estimation of both magnitude and spatial extent}

The last index was calculated by summing the magnitude of all ACSs characterised by at least $50 \%$ of pseudo-species' shifts inside a pseudo-community. This index takes into consideration both the magnitude of the shift and the number of geographical cells concerned by an ACS (Figure 4c).

\section{Literature cited}

31 Smith, T. M., Reynolds, R. W., Peterson, T. C. \& Lawrimore, J. Improvements to NOAA's Historical Merged Land-Ocean Surface Temperature Analysis (1880-2006). Journal of Climate 21, 22832296 (2008).

32 Kalnay, E. et al. The NCEP/NCAR 40-year reanalysis project. Bulletin of the American meteorology society 77, 437-470 (1996).

33 Longhurst, A. Ecological geography of the Sea. (Academic Press, 1998).

34 Cloern, J. E. et al. Biological communities in San Francisco Bay track large-scale climate forcing over the North Pacific. Geophysical Research Letters 37, L21602, doi:10.1029/2010GL044774 (2010).

35 Beaugrand, G. \& Kirby, R. R. How do marine species respond to climate change? Theories and observations. Annual Review of Marine Sciences 10, 169-197, doi:10.1146/121916-063304 (2018).

36 Beaugrand, G., Luczak, C., Goberville, E. \& Kirby, R. R. Marine biodiversity and the chessboard of life Plos One 13, e0194006, doi:https://doi.org/10.1371/journal.pone.0194006 (2018).

37 Hutchinson, G. E. Concluding remarks. Cold Spring Harbor Symposium Quantitative Biology 22, 415-427 (1957).

38 Beaugrand, G., Rombouts, I. \& Kirby, R. R. Towards an understanding of the pattern of biodiversity in the oceans. Global Ecology and Biogeography 22, 440-449 (2013).

39 Ter Braak, C. J. F. Unimodal models to relate species to environment. (DLO-Agricultural Mathematics Group, 1996).

40 Rodionov, S. A sequential algorithm for testing climate regime shifts. Geophysical Research Letters 31, L09204, doi:10.1029/2004GL019448 (2004).

41 Carpenter, S. R. \& Brock, W. A. Rising variance: a leading indicator of ecological transition. Ecology Letters 9, 311-318 (2006).

42 Carpenter, S. R. et al. Early Warnings of Regime Shifts: A Whole-Ecosystem Experiment. Science 332, 1079-1082 (2011).

43 Beaugrand, G., Edwards, M., Brander, K., Luczak, C. \& Ibañez, F. Causes and projections of abrupt climate-driven ecosystem shifts in the North Atlantic. Ecology Letters 11, 1157-1168 (2008). 
$44 \quad$ Frontier, S. Etude de la décroissance des valeurs propres dans une analyse en composantes principales: comparaison avec le modèle du bâton brisé. Journal of Experimental Marine Biology and Ecology 25, 67-75 (1976).

45 Ibanez, F. \& Dauvin, J.-C. Shape analysis of temporal ecological processes: long-term changes in English Channel macrobenthic communities. Coenoses 13, 115-129 (1998).

46 Pyper, B. J. \& Peterman, R. M. Comparison of methods to account for autocorrelation analyses of fish data. Canadian Journal of Fisheries and Aquatic Sciences 55, 2127-2140 (1998).

47 Legendre, P. \& Legendre, L. Numerical Ecology. 2 edn, (Elsevier Science B.V., 1998).

48 Goberville, E., Beaugrand, G. \& Edwards, M. Synchronous response of marine plankton ecosystems to climate in the Northeast Atlantic and the North Sea. Journal of Marine Systems, doi:10.1016/j.jmarsys.2013.05.008 (2013).

49 Beaugrand, G. \& Ibañez, F. Spatial dependence of pelagic diversity in the North Atlantic Ocean. Marine Ecology Progress Series 232, 197-211 (2002). 



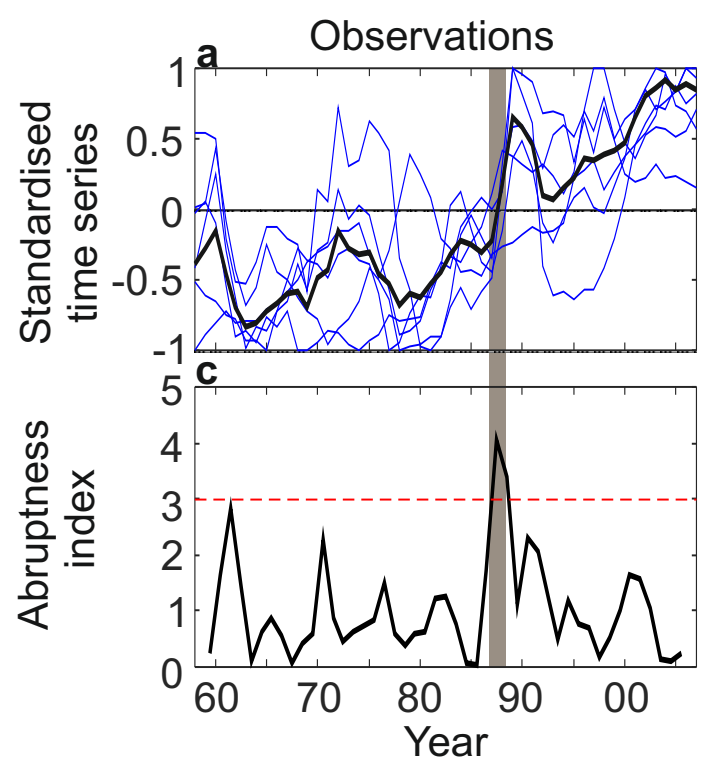

$43 \%$ (6) of the 14 species/taxa are related to the observed first PC (blue in pannel a)

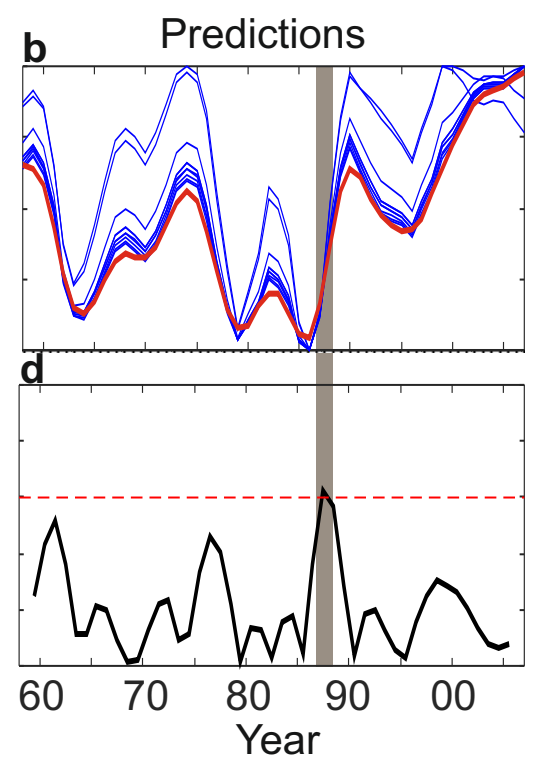

$71 \%$ (10) of the 14 pseudospecies are related to the simulated first PC (blue in pannel b)

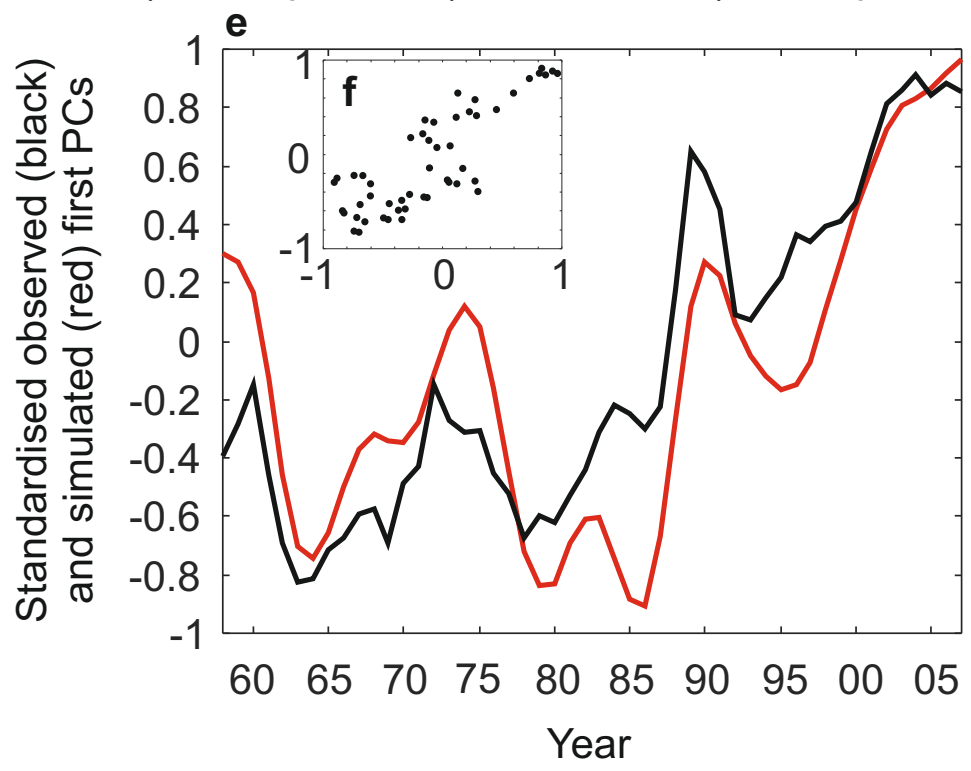



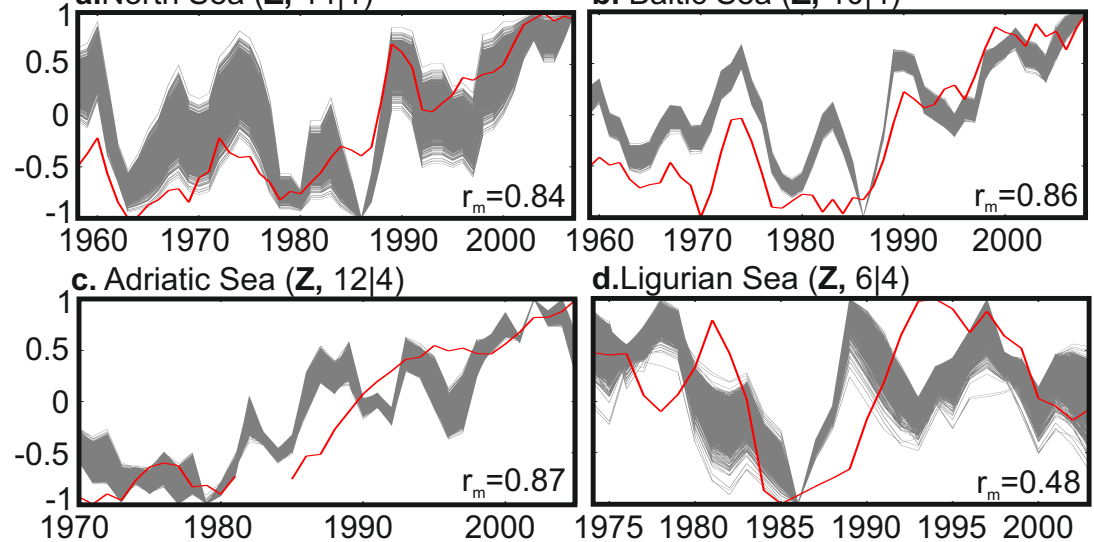

d.Ligurian Sea $(\mathbf{Z}, 6 \mid 4)$

e. Northwest Atlantic (south)(Z,17|6) f. Northwest Atlantic (north)(Z, F, 24|6
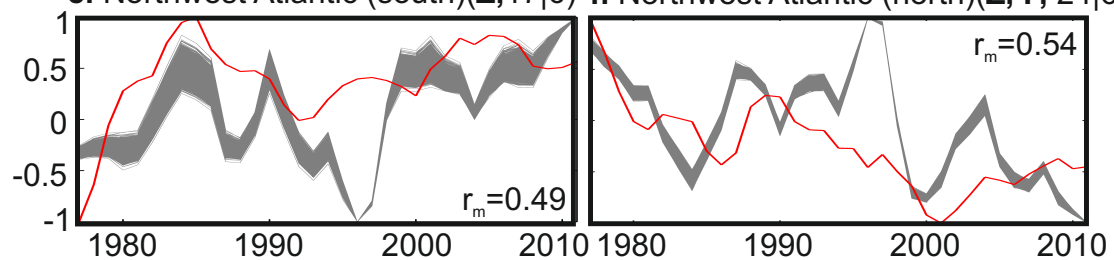

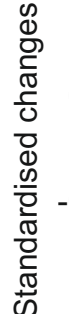

g. Transition zone $(\mathbf{Z}, 67 \mid 2)$

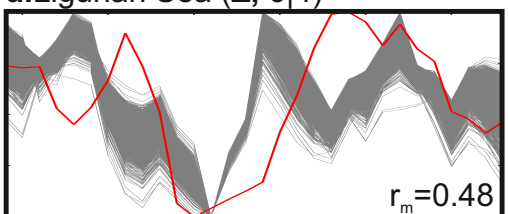

h. Oyashio $(Z, 32 \mid 2)$

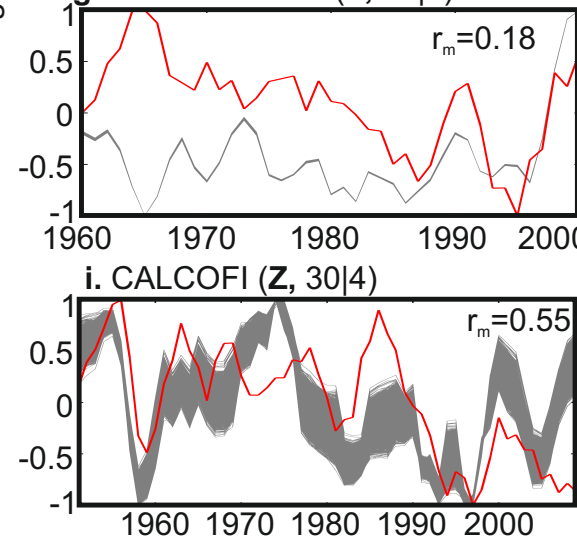

k. East Pacific (F, 24|1)
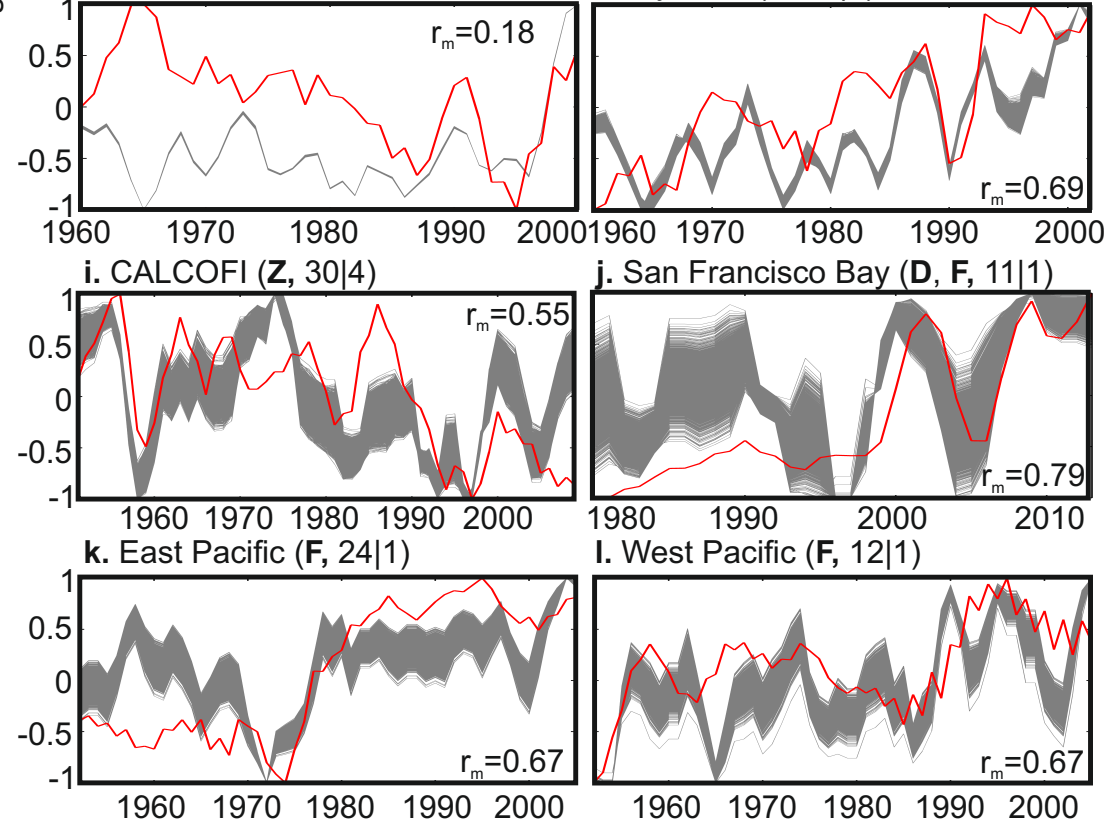

j. San Francisco Bay (D, F, 11|1)

m. $\operatorname{HOT}(\mathbf{Z}, 30 \mid 6)$
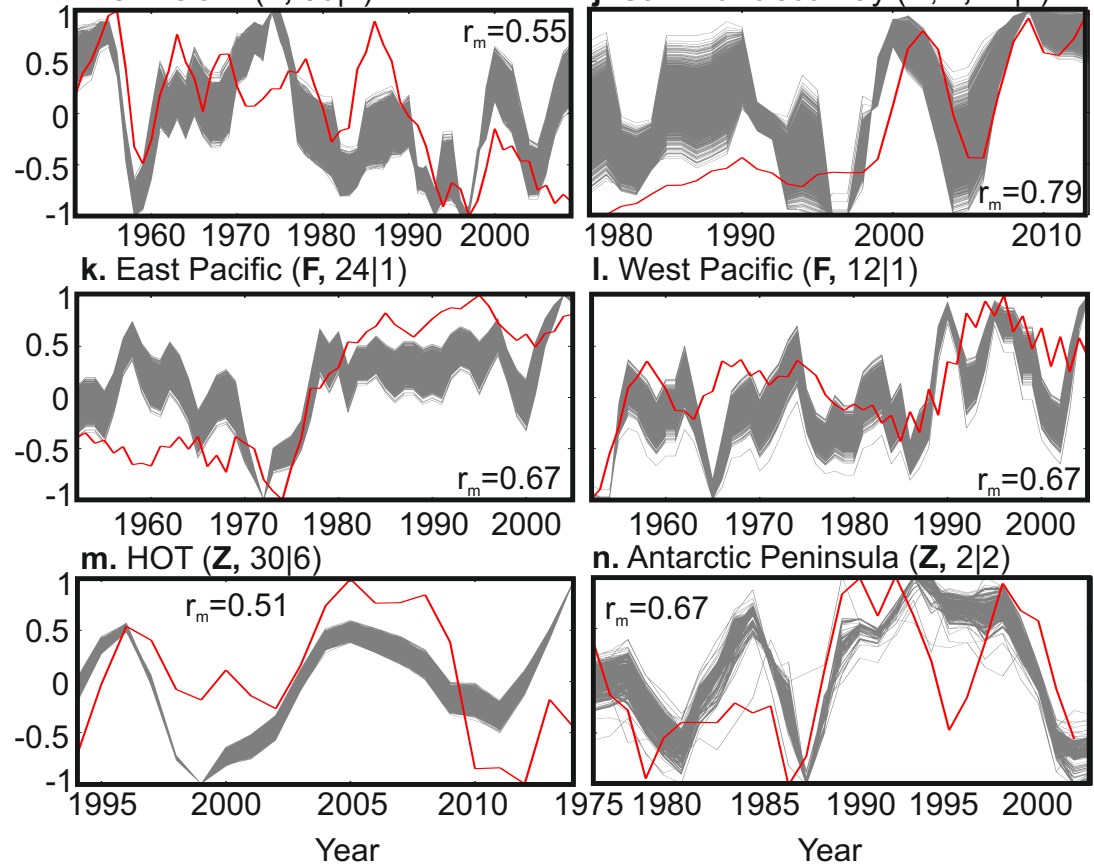

I. West Pacific (F, 12|1)

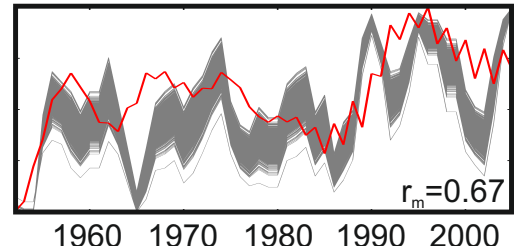

n. Antarctic Peninsula (Z, 2|2)

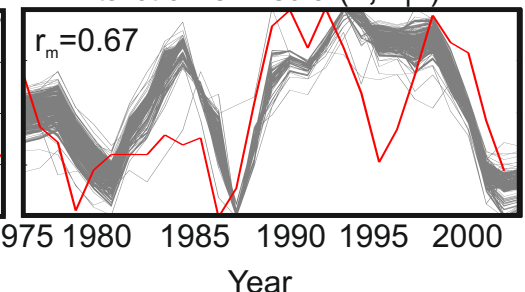

o. Scatter plot

p. Correlation
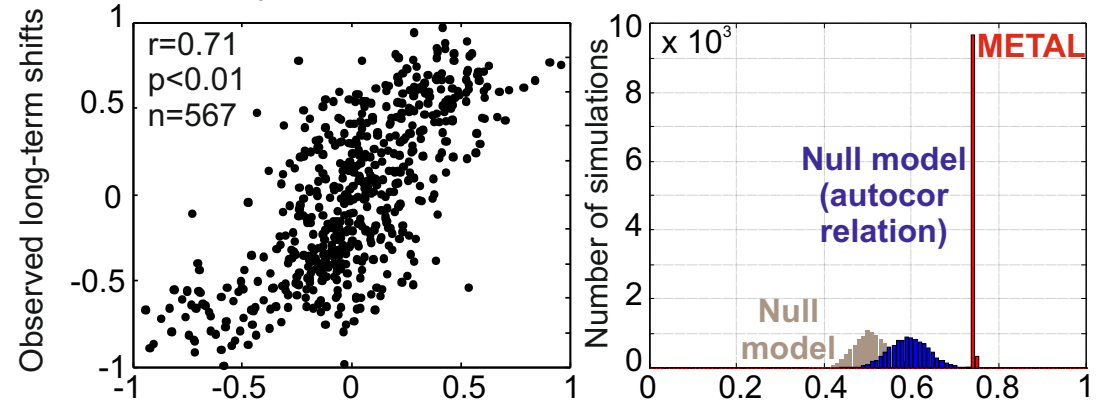

Predicted long-term shifts

Correlation 
Long-term community shift
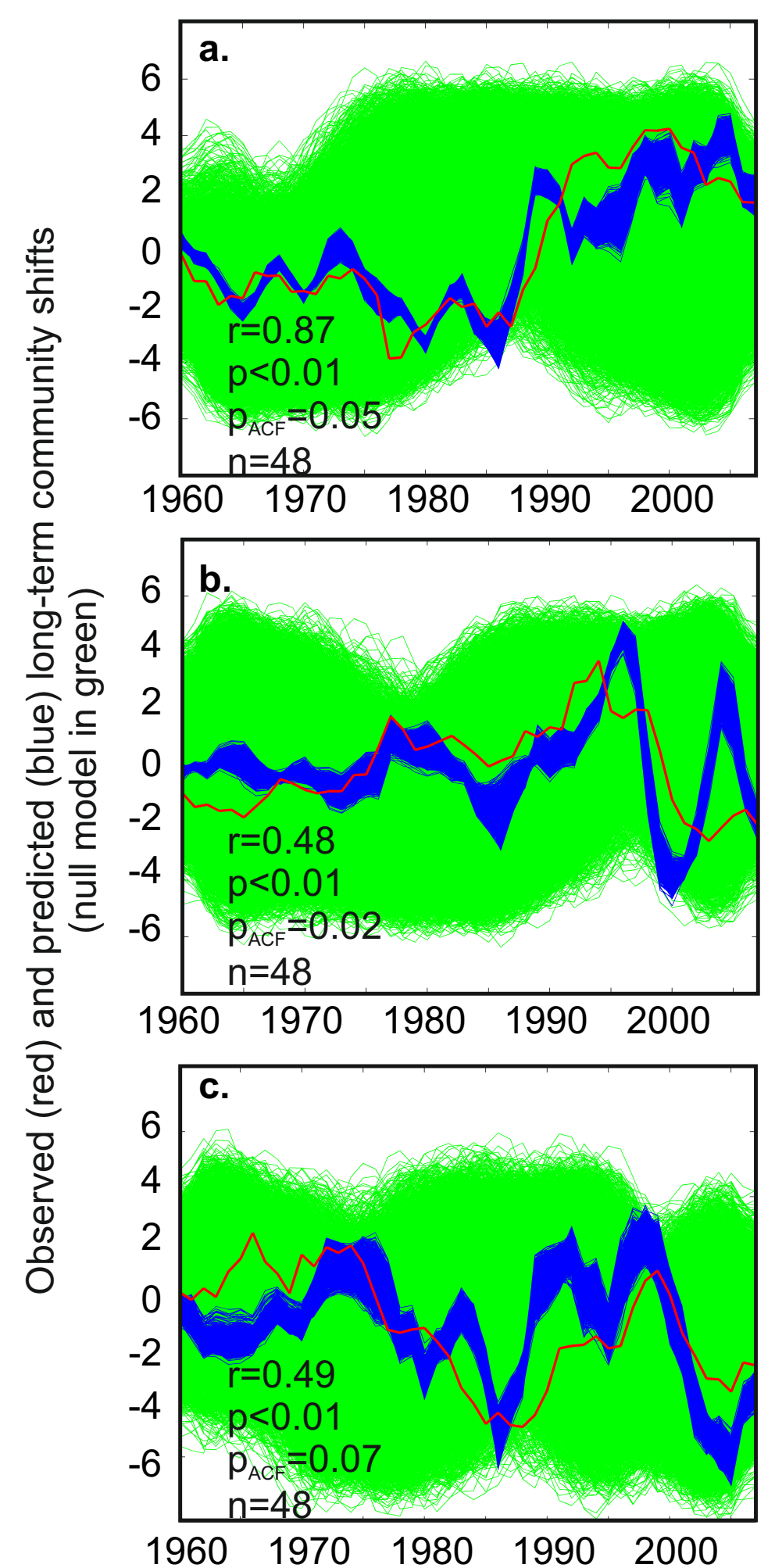

Abrupt shift
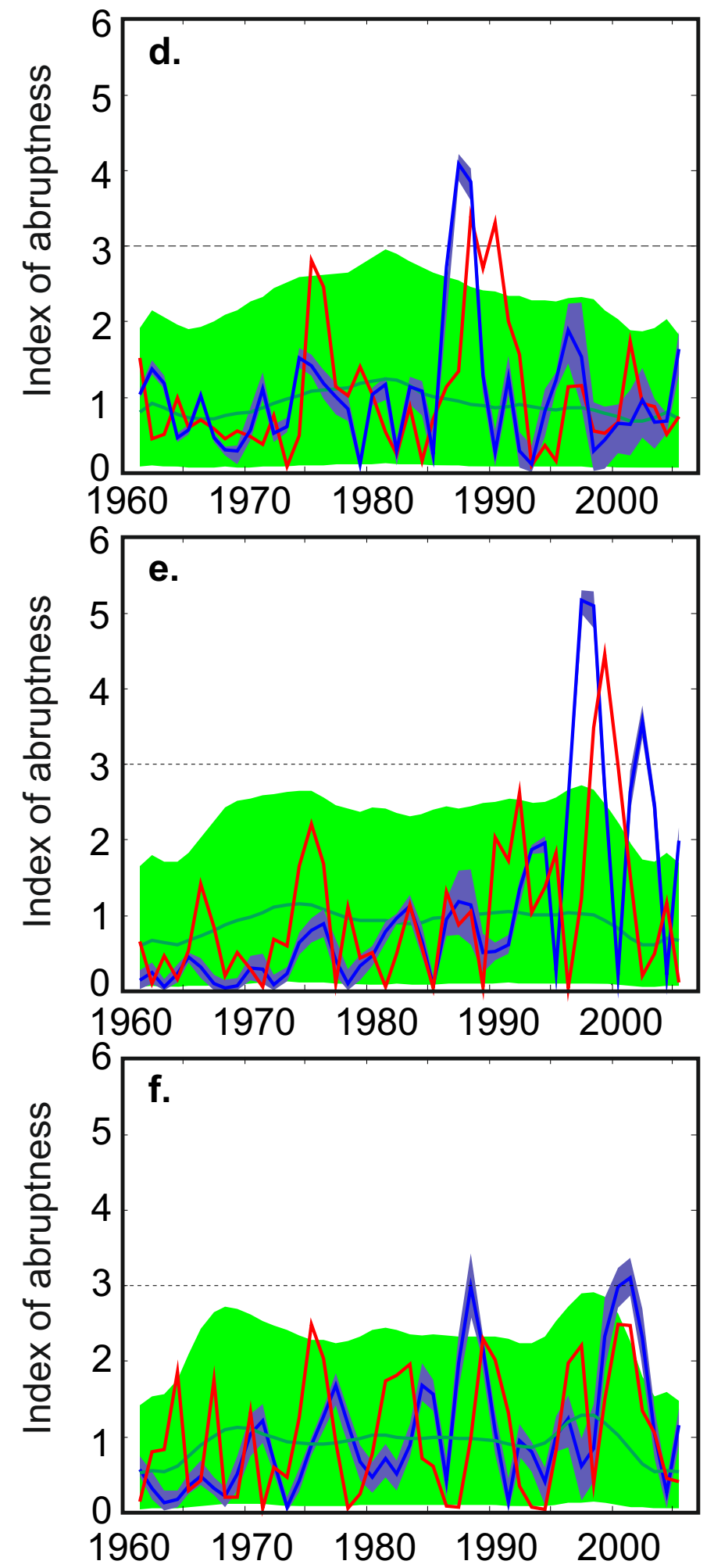


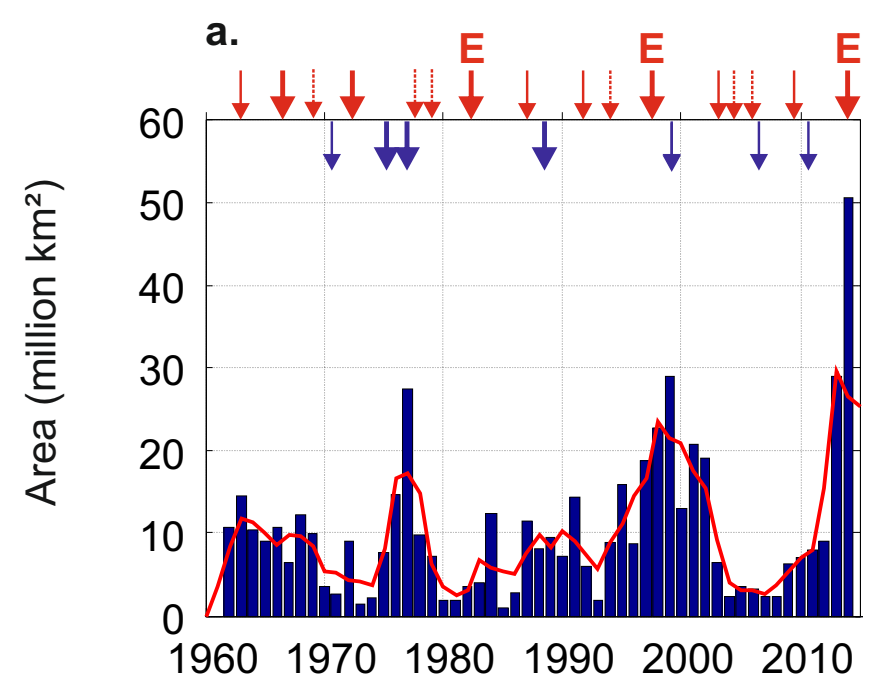

b.
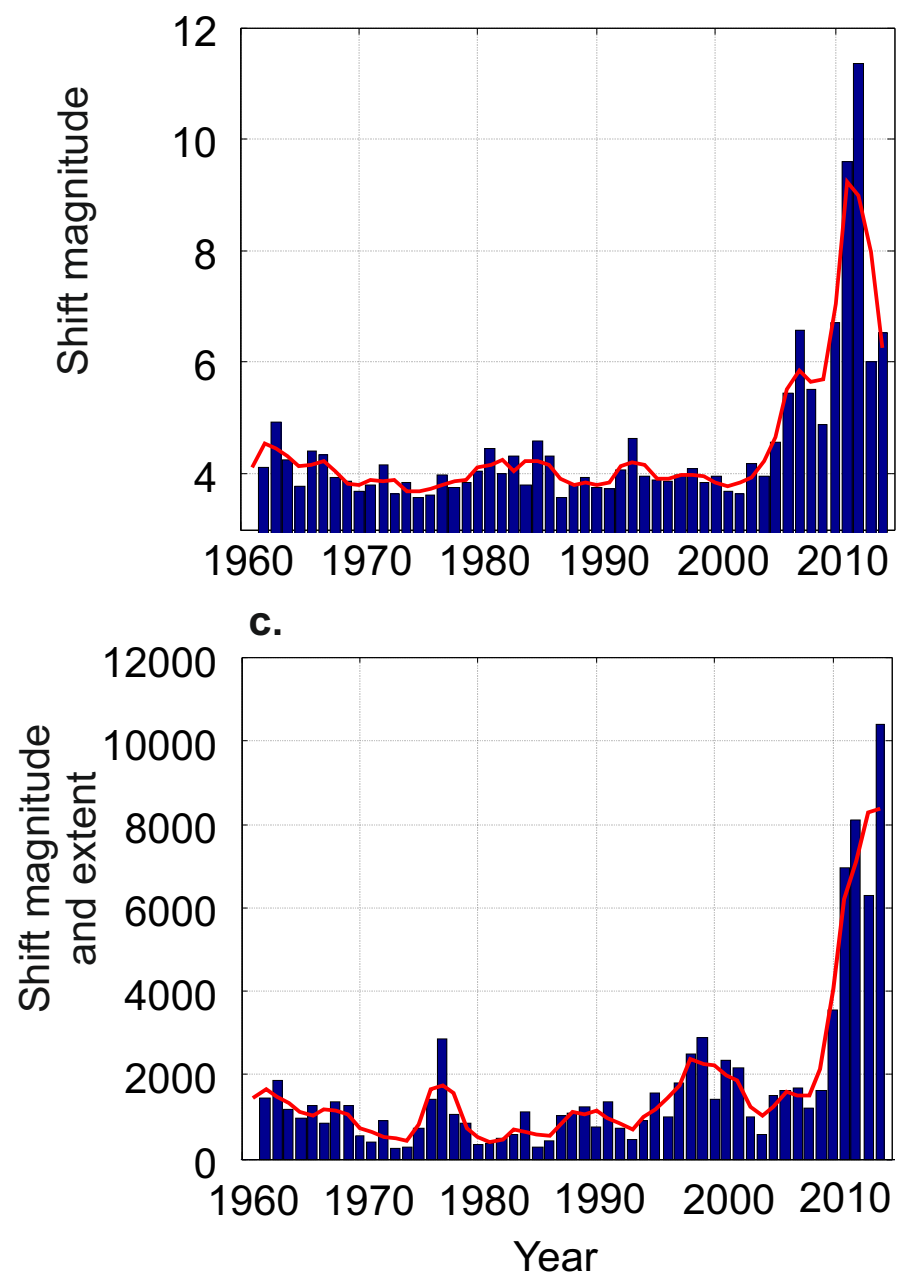
1975-1979
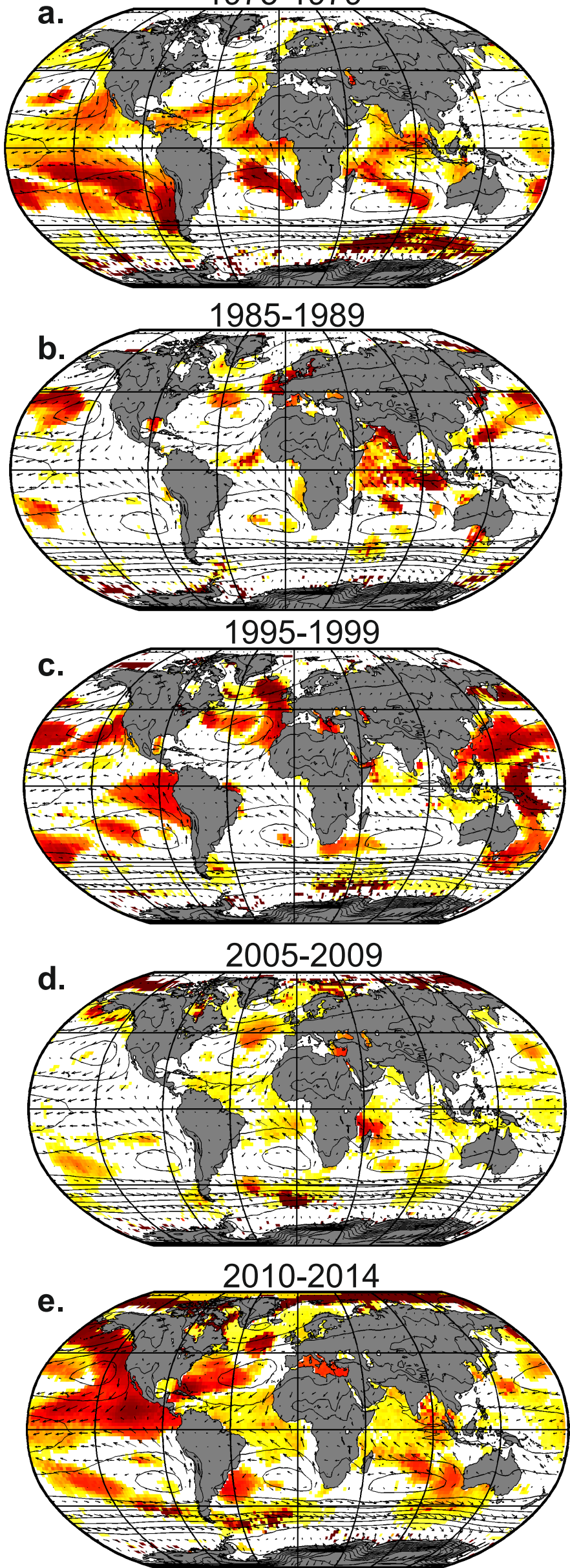

$\begin{array}{llllll}0 & 20 & 40 & 60 & 80 & 100\end{array}$
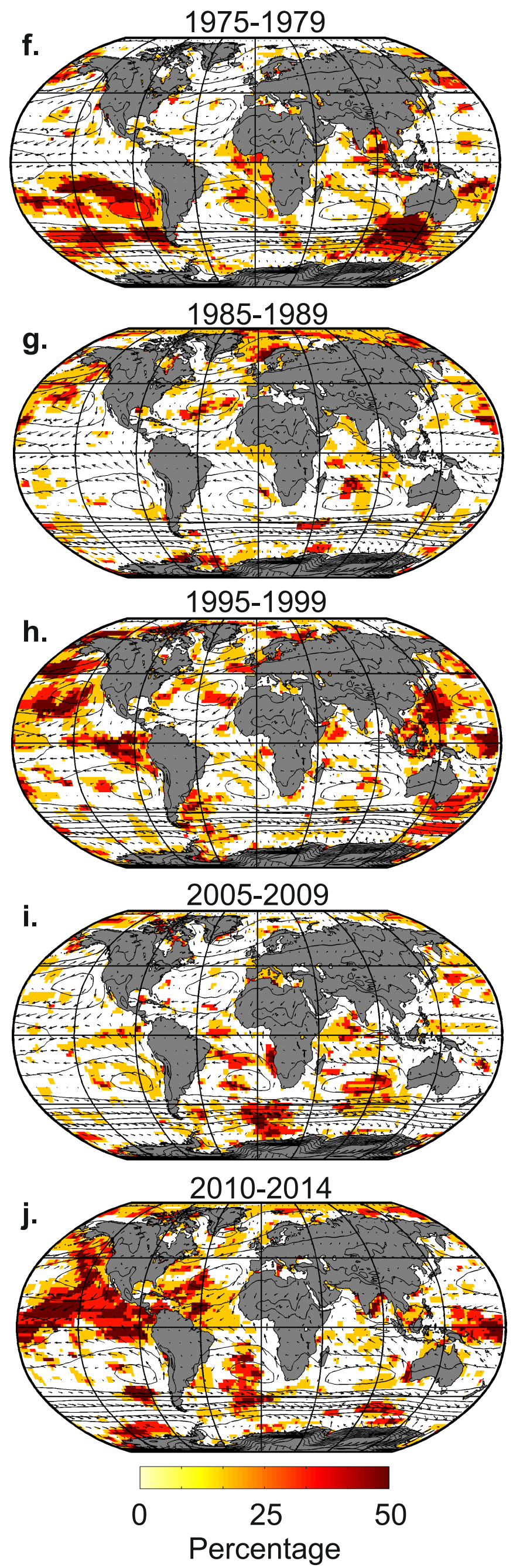


\section{Supplementary Information}

\section{Prediction of unprecedented biological shifts in the global ocean}

G. Beaugrand ${ }^{1,2^{*}}$, A. Conversi ${ }^{3,4}$, A. Atkinson ${ }^{5}$, J. Cloern $^{6}$, S. Chiba ${ }^{7}$, S. Fonda-Umani ${ }^{8}$, R.R. Kirby $^{9}$, C.H. Greene ${ }^{10}$, E. Goberville ${ }^{11}$, S.A. Otto ${ }^{12}$, P.C. Reid ${ }^{2,4}$, L. Stemmann ${ }^{13}$, M. Edwards ${ }^{2,4}$

* Corresponding author: gregory.beaugrand@cnrs.fr

${ }^{1}$ Centre National de la Recherche Scientifique, Laboratoire d'Océanologie et de Géosciences' UMR LOG CNRS 8187, Station Marine, Université des Sciences et Technologies de Lille 1 Lille 1 BP 80, 62930 Wimereux, France

${ }^{2}$ Marine Biological Association, the Continuous Plankton Recorder (CPR) survey, The Laboratory, Citadel Hill, The Hoe, Plymouth PL1 2PB, UK

${ }^{3}$ ISMAR-Marine Sciences Institute in La Spezia, CNR-National Research Council of Italy, Forte Santa Teresa, Loc. Pozzuolo, 19032, Lerici, SP, Italy

${ }^{4}$ School of Biological \& Marine Sciences, University of Plymouth, Plymouth, PL4 8AA, UK

${ }^{5}$ Plymouth Marine Laboratory, Prospect Place, The Hoe, Plymouth PL13DH, UK

${ }^{6}$ USGS MS496 345 Middlefield Rd., Menlo Park, CA, 94025, USA

${ }^{7}$ RCGC, JAMSTEC, 3173-25 Showa-machi, Kanazawa-ku, Yokohama, 236-0001 Japan

${ }^{8}$ Department of Life Sciences, University of Trieste, v. Giorgieri, 10, 34127 Trieste, Italy

${ }^{9}$ The Secchi Disk Foundation, Kiln Cottage, Gnaton, Yealmpton, PI8 2HU, UK

${ }^{10}$ Ocean Resources and Ecosystems Program, Cornell University, Ithaca, NY 14853-1504, USA

${ }^{11}$ Unité Biologie des organismes et écosystèmes aquatiques (BOREA), Muséum national d'Histoire naturelle, Sorbonne Université, Université de Caen Normandie, Université des Antilles, CNRS, IRD, CP53, 61 rue Buffon 75005 Paris, France

12 Institute of Marine Ecosystem and Fishery Science (IMF), Center for Earth System Research and Sustainability, University of Hamburg, 22767 Hamburg, Germany

${ }^{13}$ Sorbonne Université, Institut de la Mer de Villefranche, CNRS, UMR 7093, Laboratoire d'Océanographie de Villefranche, F-06230, Villefranche/mer, France 


\section{Introduction}

This piece provides details on the data and on the background work supporting the main article, including a number of statistical tests and validations meant to provide the reader with confidence on the METAL ${ }^{1}$-based framework used. A glossary with the list of the acronyms used in the text is available in Supplementary Table 1. The observed data time series are described in Supplementary Tables 2-3, Supplementary Figure 1, and in Methods. Supplementary Figure 2 shows the relationships between observed and predicted long-term biological changes, with predictions originating from a null model that results from the standardised Principal Components Analysis (PCA) of randomly generated time series with an order- 1 temporal autocorrelation $\geq 0.5$ carried out for each system. Supplementary Figure 3 shows how/why years were chosen to perform the 'global' PCAs. The METAL-based abrupt shifts predicted at global-scale for the marine community are shown at 3-yearly intervals from 19601963 to 2012-2015 in Supplementary Figure 4, while the abrupt shifts in observed climate parameters are shown in Supplementary Figure 5 for the same period and scale. Supplementary Table 4 shows the percentage of variance explained by the first two axes of the principal component analyses performed on observations and the variance predicted from a broken-stick distribution. Background statistical information on observed and predicted Principal Components is presented in Supplementary Tables 5-6, while Supplementary Table 7 shows the linear correlations between the spatial extent of predicted abrupt community shifts from 1960 to 2015 and the spatial extent of significant abrupt climatic shifts based on six climatic parameters. Supplementary Figure 6 shows the spatial patterns of abrupt shifts of six climatic parameters centred on the period 2010-2014. Supplementary Figure 7 shows a summary of the METAL procedures that lead to the building of each local pseudo-community, the test of our framework against observed data and the prediction of abrupt community shifts. The detection ability of the abrupt shift algorithm is shown in Supplementary Figures 8-12 using simulated times series of growing complexity. Supplementary Figure 13 shows the autocorrelation of the time series used to test the algorithm of abrupt shift detection. Supplementary Figures 14 described the main numerical procedures used in Figures 2 and 3. Supplementary Note 1 discusses the limitations of our framework and of some statistical procedures. Supplementary Note 2 describes information on biological sites. Supplementary Note 3 summarizes the MacroEcological Theory on the Arrangement of Life. Supplementary Note 4 summarizes the test of the abrupt shift detection algorithm.

\section{Supplementary Notes}

\section{Supplementary Note 1| Limitations of our procedures}

\section{Principal Component Analyses}

As with all statistical analyses, Principal Component Analyses (PCAs) have some limitations ${ }^{1}$. Although some parts of the signal in the first two PCs may have been influenced by the noise associated to biological time series ${ }^{1}$, we are confident that this stochasticity did not alter our conclusions because (i) we used null models with and without autocorrelation, which showed that patterns in observed and predicted biological shifts were far from random (Figures 1-3, Supplementary Figure 2); (ii) Most longterm changes were significantly correlated with predictions at the scale of a marine system and globally (Figures 1-3); indeed correlations were significant after accounting for temporal autocorrelation and also substantially higher than correlations based on null models (Figures 1-3, Supplementary Figure 2); (iii) our framework, not based on PCA (Figure 5), predicted well all shifts reported in the mid-1970s (north-east Pacific and tropical), the mid-1980s (European Seas) and the mid-1990s (e.g. north-east

\footnotetext{
${ }^{1}$ MacroEcological Theory on the Arrangement of Life
} 
Atlantic); (iv) the last two shifts were also detected using time series analyses and not ordination techniques in this study (Figure 5 ) and others ${ }^{2-4}$.

We applied a test based on a broken-stick distribution ${ }^{5}$ to evaluate the significance of the first two axes of the PCAs. With the exception of three eigenvalues, this analysis revealed that $89 \%(25)$ of the first two observed eigenvalues were significant (Supplementary Table 7; see Figure 2); the nonsignificant eigenvalues (PCS) were not selected in Figure 2a-n because the correlation with pred-PCs was low.

\section{Representativity of the monitoring sites to test our global-scale model}

Any proposed global frameworks, including global biogeochemical models, are likely to suffer from lack of representativity, especially when long-term time series are needed. We have done our best to select time series representative of different biomes. Data used in the paper are well representative of the long-term datasets currently available in pelagic ecosystems ${ }^{6,7}$. Our modelled data will be available through our website (http://metaltheory.weebly.com/) to allow researchers to test our theory using their datasets.

Although we do not cover all oceanic regions (e.g. South Pacific and Atlantic, Indian Ocean), areas chosen to calculate long-term changes in community have a large bathymetry range (Supplementary Figure 15). The histogram, based on the geographical limits shown in Supplementary Table 3 (last column), shows that we cover continental shelves and oceanic areas; selected oceanic areas (i.e. water depth $>200 \mathrm{~m}$ ) and continental shelves (i.e. water depth $<200 \mathrm{~m})$ represented 7.5 million $\mathrm{km}^{2}(2.25 \%$ of the total oceans) and 2.23 million $\mathrm{km}^{2}$ ( $8.04 \%$ of the total continental shelves), respectively. These area calculations are based on the regions selected for the pseudo-communities, which are larger than observation sites. However, because they were frequently correlated (Figures 1-3), we assumed that the areas we chose to test our framework represented at best $2.25 \%$ and $8.04 \%$ of the total oceans and continental shelves, respectively.

Although our framework is applied on a global scale (Figures 4 and 5), it was tested on a restricted section of the global ocean as such a global monitoring network does not exist (Supplementary Figure 1). However, we considered the sampling spatial coverage representative of environmental conditions from oceanic to neritic and from tropical to polar. In this section, we tested whether the correlation was related to the characteristics of a region (Supplementary Figure 16). We represented the correlation between observed and predicted principal components (PCs of Figure 2a-n) as a function of the surface area and the mean bathymetry of each region (Supplementary Figure 16). Although correlations are highest for shallow regions, there are places where both low and high correlations can be observed for similar bathymetry and surface area everywhere; this is the case from high bathymetry (Oyashio versus transition zone) to medium (California Current versus East Pacific Rim) and low bathymetry (northern part of the north-west Atlantic versus Adriatic Sea). This analysis did not provide evidence against an application of our framework in remote oceanic areas, although more tests in other regions will definitively reinforce it.

\section{Test of METAL performance in offshore areas of the North Atlantic Ocean}

To further test whether our framework could be extended to deep-sea/open oceanic regions (i.e. areas below $200 \mathrm{~m}^{8}$ or corresponding to case 1 waters ${ }^{9}$ ), we used a separate subset of data from the Continuous Plankton Recorder (CPR) survey. We chose this survey because it provides consistent, multi-decadal plankton sampling over a large number of areas, spanning from near-coast to open waters and from shallow to deep waters ${ }^{10,11}$. We selected five offshore regions well covered by the CPR survey (i.e. number of samples $>5000$ samples; Supplementary Figure 17, left inner panels). We included zooplankton species that had an annual abundance $>0$ in $\geq 20$ years during the period 19582016 and used the procedure applied previously to compare long-term changes in biological and 
theoretical communities (Supplementary Figure 7 and Figures 1-2). Long-term observed community changes were highly positively correlated with predicted biological changes from our model for the time period 1958-2016 in all five regions (Supplementary Figure 17, left panels). All predicted and observed communities exhibited a pronounced shift in the middle to the end of the 1990s. Such a shift has been reported in the Celtic Sea, the Bay of Biscay and oceanic areas located to the west of the British Isles ${ }^{3,12}$. High values of the principal components were found at the beginning of the time series in northern regions (e.g. Denmark Strait and Faroe-Iceland Rise). Cumulative frequency histograms (middle and right panels) show that $>\sim 90 \%$ of samples used to estimate long-term community changes in the five regions were collected in regions with water depth $>200 \mathrm{~m}$ (i.e. the shelf edge and the ocean) and in regions $>100 \mathrm{~km}$ from land (Supplementary Figure 17, middle panels). We are therefore confident that our framework is applicable more generally to oceanic regions including the open ocean, despite the fact that our framework was not tested in some oceanic basins such as the South Atlantic, Pacific and Indian Oceans.

\section{Long-term changes in the 14 systems unexplained by our model}

Although the predicted data provided overall very good significant correlations with observed data, higher residual variability was observed occasionally in some areas such as in the Western Pacific Transition Zone (Figure $2 \mathrm{~g}$ ) and may reflect any of three main causes. First, it may be related to local environmental complexity that is not fully resolved in our model. Future improvements of the METAL theory may help reduce this variability by including further ecological factors ${ }^{13}$. At present however, ecological dimensions of interest are rarely available on a year-to-year basis. Second, it is likely that many marine ecosystems, especially coastal ones, are also influenced by human-induced factors such as overfishing, eutrophication and pollution, and these probably affect the biological composition of communities ${ }^{14,15}$. Third, it is possible that uncertainties in sampling procedures significantly affect the time series, although the PCA helped to reduce this variance ${ }^{16}$.

\section{The West Pacific transition Zone}

The low correlation found in the West Pacific Transition Zone (zone 11 in Figure $2 \mathrm{~g}$ ) is probably related to its sampling spatio-temporal variability ${ }^{17}$ or its high hydro-dynamic complexity ${ }^{18}$. We have investigated whether this absence of a correlation was constant throughout the time series (19602000) or whether it was related to a specific event. To do so, we calculated the correlations by progressively removing observations (years) from the beginning to the $31^{\text {st }}$ observation. The first 5 years were badly reproduced by the model, being off-phase (Supplementary Figure 18). When these years were removed, the correlation improved and became close to the level of significance after adjusting the degree of freedom to account for temporal autocorrelation (Supplementary Figure 18, left lower and upper panels). A plateau was reached after removing the first 24 years (Supplementary Figure 18, right lower and upper panels); correlations became significantly high. This was primarily due to the presence of two substantial shifts observed at the end of the 1980s and 1990s that were well reproduced by our framework (Figure $2 \mathrm{~g}$ and Supplementary Figure 18). Although the year-to-year variability were not well reproduced at the beginning of the time series, the two substantial shifts observed at the end of the 1980s and the 1990s were captured by our model.

\section{The 1976/1977 North Pacific shift}

Based on 69 biological and 31 climatic time series, Hare and Mantua ${ }^{19}$ examined long-term changes in the north-east Pacific from 1965 to 1997. They found what they termed a regime shift (i.e. a sudden, substantial and temporally persistent changes in the state of communities/ecosystems ${ }^{20}$ ) in $1976 / 1977$, which was latter supported by a number of studies ${ }^{21}$. The 1976/1977 shift was not identified in Figure 3 because the number of systems in the north-east Pacific were limited in our 
analyses. However, other results are in agreement with what is currently known about this shift. For example, Hare and Mantua ${ }^{19}$ found that the regime shift was more detected on biological than climatic time series, the latter being more noisy than regime-like ${ }^{19}$. It is interesting that in our study no abrupt climatic shifts were detected because changes were more gradual (Figure 5f). This situation is well explained in the example provided in Supplementary Figure 10 when time series are mainly composed of cyclical or pseudo-cyclical and "noisy" variability. (It is also well-documented that shifts are more easily explained by large-scale hydro-climatic indices than by local hydro-climatic time series ${ }^{22}$.) Applied on pseudo-species, our procedure revealed that between 20 (yellow in Figure $5 a$ ) and $40 \%$ (orange in Figure $5 \mathrm{a}$ ) of the pseudo-species exhibited a significant shift in the area covered by Hare and Mantua ${ }^{19}$. Although abrupt shifts were not detected on the local hydro-climatic time series (Figure 5f), many pseudo-species exhibited a substantial abrupt shift (Figure 5a). Our model therefore suggests that the non-linear interaction between the niche of species and environmental fluctuations is the mechanism by which biological systems amplify weak environmental fluctuations ${ }^{23}$. When a PCA was applied on pseudo-species, the first PC revealed the 1976/1977 shift (Figure 2k). Finally, the substantial spatial extent of the 1976/1977 shift was also clearly identified (see Figure 4).

\section{Is our framework mechanistic or correlative?}

Our framework is mechanistic rather than empirical (correlative or phenomenological). Empirical (correlative) models are based on direct observations or measurements. In contrast, mechanistic models are based on an understanding of the system's behaviour. Here, our model based on the METAL theory ${ }^{24-26}$ was fully independent on the biological data and no observations were needed for calibration. We generated pseudo-species that had their own thermal niche and species interacted subsequently with local fluctuations in the thermal regime. At this organisational level, we assumed that the ecological niche is an elementary macroscopic foundation, which integrates mechanisms that are controlled by the genome fixing individual's physiology ${ }^{26}$. Based on the same model (with no adjustment of any parameters whatever the system under consideration), we reproduce most longterm changes well, including abrupt community shifts, observed in 14 marine pelagic ecosystems ranging from tropical to temperate and polar regions.

\section{Strengths and limitations of the use of a single parameter (temperature)}

We assume here that the main driver by which atmospheric forcing may affect biological communities is sea temperature because many studies have shown that this parameter has a cardinal influence on species physiology, biology and ecology ${ }^{27-29}$. Temperature controls all biological processes from the molecular to the cell and the organism levels ${ }^{28}$. Temperature alters growth, reproduction, mortality, the behaviour of organisms at the species level and biotic interactions (positive or negative) at the community level ${ }^{30,31}$. At a global scale, temperature alterations can influence the ocean deep and surface circulation, and largely determine the location of biogeographic provinces and biomes. Temperature patterns modulate ecological services such as food production and carbon sequestration that marine ecosystems provide to humanity ${ }^{32,33}$. It is therefore not surprising that many biogeographical studies have revealed a cardinal influence of temperature on marine biodiversity ${ }^{34-38}$.

However, we are aware that multiple environmental parameters influence the productivity and distributions of individual marine species, and their grouping together as communities and ecosystems ${ }^{13}$. Nutrients and light limit phytoplankton production ${ }^{39,40}$. Both bathymetry and local spatial variability in bathymetry are key determinants of marine pelagic biodiversity ${ }^{41}$. Dissolved oxygen must remain high enough to support respiration ${ }^{42}$. Mixed Layer Depth (MLD) is an important parameter for phytoplankton production and controls the spatial distribution of many plankton species ${ }^{39,43}$. Oceanic $\mathrm{pH}$ influences calcifying organisms such as coccolithophorids, foraminifers, corals and pteropods ${ }^{44,45}$. Wind intensity, affects prey-predator encounter rates ${ }^{46}$ by its effects on oceanic turbulence, and nutrient supply rates by its effects on vertical mixing ${ }^{43}$. Wind direction, by its control 
of the distribution of some meroplankton species might strongly affect recruitment of some benthic organisms ${ }^{47}$. Additional anthropogenic factors such as overfishing may compound synergistically with natural factors to drive ecosystem shifts ${ }^{48,49}$. The above list is far from exhaustive, but shows the complexity of pathways and types of control that the environment might exert on organisms and biocoenoses.

Future versions of our framework should therefore consider several environmental parameters simultaneously. However, this may not improve significantly the model because many marine environmental parameters covary with temperature (e.g. ice, oxygen, some nutrients). Furthermore, many environmental parameters are not available with sufficient accuracy on a global scale and on a year-to-year basis. That is why we have first focused on temperature.

Our model cannot realistically implement biotic interactions in the construction of pseudocommunities on a global scale, although multiple examples suggest that they can be quite important in some ecosystems ${ }^{31,50}$. There are currently insufficient data to include these potentially important top-down and competitive interactions over the scales needed. Therefore, our overall approach was to test temperature to examine how well this factor alone could modulate global-scale pelagic assemblages, leaving scope for future refinement. We are aware, however, that at a more local scale, biotic interactions may precipitate the climatic effects on pseudo-communities ${ }^{51}$.

\section{Terminology}

All biological systems do not persist in total stasis for long and it is just a matter of time before they change. Here, we talk about long-term community change to characterise any type of alteration in a pseudo-community and Abrupt Community Shift (ACS) when a rapid and substantial shift takes place.

In the scientific literature, the terminology used to qualify shifts in a community includes regime shift ${ }^{52,53}$, critical transition ${ }^{14}$, abrupt shift ${ }^{54}$, abrupt transition ${ }^{55}$, acceleration phases, stepwise change ${ }^{56}$, phase transition ${ }^{57}$, phase shift, state shift ${ }^{58}$, system flips ${ }^{59}$, stark changes ${ }^{60}$, and catastrophic shift ${ }^{61}$. Although sometimes seen as synonymous, at least two types of terms focus on different aspects. The first category (regime, phase and state shift as well as phase transition) puts an emphasis on the dynamic regime or dynamic equilibrium of systems. These concepts are defined as a substantial and rapid shift between two contrasting persistent states ${ }^{20}$. These terms assume the existence of stable states. After the shift, the system has a different mean and sometimes an altered variance but the variability around the mean is assumed to be stationary (i.e. no temporal autocorrelation and no cyclical variability). Many statistical tests can be used to identify this type of shift. The sequential t-test of Rodionov ${ }^{62,63}$ has been frequently applied ${ }^{3}$ but many other techniques such as split-moving window boundary analysis ${ }^{64}$ may also be used ${ }^{56}$. When the table contains many variables, (chronological or not) cluster analysis or principal component analysis have also been used ${ }^{19,65}$. Note that other techniques can be applied ${ }^{56,66,67}$, including change-point analysis ${ }^{68}$.

The second category (abrupt shift or transition, acceleration phases, stark changes, catastrophic shifts, critical transition) focuses mainly on the shift, which makes the emphasis on the variance at the time of the shift and not the state. They are sometimes called vacillations in climatology ${ }^{69}$. These terms are interesting because there is no need to invoke a stable state or the persistence of a regime. Such shifts may be better defined by their variance signature ${ }^{54}$. All sources of variability can coexist and periods of slow and fast variance are often observed ${ }^{54}$. Detection of these shifts can be accomplished by the use of the coefficient of variation, the measure of the autocorrelation, or the quantification of the multi-scale variance along the time series ${ }^{54}$. Change-point analysis may also be used to detect such shifts. Principal components analysis, often used in this research field, is also adapted to detect changes in mean and the increase of variance at the time of the shift. 
In this study, we have used the terms Abrupt Community Shift (ACS) instead of regime shift because the former term places the emphasis on the variance at the time of the shift and not the state. There is no need to invoke a stable state or the persistence of a regime. Therefore, ACSs simply reflect rapid alterations in species composition that may significantly affect biodiversity with potential consequences for ecosystems services. This definition is more general and has no a priori requirement to invoke mechanisms behind the origin of those shifts (e.g. double-integration hypothesis, alternative stable states $)^{4,14,70}$.

\section{Supplementary Note 2| Information on biological variables}

Below, we summarise the main characteristics of the 14 selected regions (see also Supplementary Figure 1 and Supplementary Table 2 for more details). Biological variables (e.g. species, taxon, biomass, size fraction) varied from one region to another (see Methods section and Supplementary Tables 2-3). For each system, all available biological data were used. A thorough description of species composition in each system can be found in Beaugrand and colleagues $^{71}$, Cloern and colleagues ${ }^{72}$, Landry and colleagues $^{73}$ and Atkinson and colleagues ${ }^{74}$.

1. The North Sea (zooplankton). The Continuous Plankton Recorder (CPR) survey is the largest multi-decadal plankton monitoring programme in the world ${ }^{10}$. The CPR is towed behind ships of opportunity on their normal trading routes at usually 15-20 knots speed. The routes are repeated in most cases with monthly frequency, and have varied somewhat over time. The self-contained automatic plankton recorder collects plankton continuously from a standard depth of about $7 \mathrm{~m}^{75}$. Water enters the CPR through a square aperture at the front with sides of $1.27 \mathrm{~cm}\left(1.61 \mathrm{~cm}^{2}\right)$ and exits through the rear of the device. In-between, the plankton in the water is filtered onto a constantly moving band of silk. Sampling is performed on a monthly basis. The PCA was performed at an annual scale using 14 zooplankton species for the 50 -year period $1958-2007^{71}$. The first two PCs were retained and explained $27.96 \%$ and $27.15 \%$ of the variance, respectively. For this work we have used the area enclosed in the North Sea, from 1958-2007 (53645 samples), and the taxa Acartia spp., Calanus finmarchicus, Calanus helgolandicus, Candacia armata, Centropages hamatus, Centropages typicus, Labidocera wollastoni, Metridia lucens, Para-pseudocalanus spp., Pseudocalanus elongatus adult, Temora longicornis, total Harpacticoida, Corycaeus spp., Oithona spp.

2. The Central Baltic Sea (zooplankton biomass). Data on biomass of major mesozooplankton species were derived from a database of the Latvian Institute of Food Safety, Animal Health and Environment BIOR covering the Gotland basin. The Latvian dataset is the longest and most consistent dataset in the Baltic Sea. Individual hauls were carried out to a maximum depth of $100 \mathrm{~m}^{76}$. Sampling takes place on a seasonal basis and the PCA was performed at a seasonal scale using 10 zooplankton species for the 52-year period $1957-2008^{71}$. The first two PCs were retained and explained $23.08 \%$ and $11.12 \%$ of the variance, respectively. For this work we have used seasonal mean abundances (ind $/ \mathrm{m}^{3}$ ) of Acartia spp., Bivalvia, Centropages hamatus, Evadne nordmanni, Fritillaria borealis, Podon spp., Polychaeta, Pseudocalanus acuspes, Synchaeta spp., Temora longicornis.

3. The Ligurian Sea, Western Mediterranean Sea (zooplankton abundance and biovolume). The Villefranche Point B dataset consists of more than 50 years of samples collected off Villefranche in the Ligurian Sea (western Mediterranean) at $43^{\circ} 41^{\prime} \mathrm{N}, 07^{\circ} 19^{\prime} \mathrm{E}$. Samples were collected daily by a vertical tow from bottom to surface $(75-0 \mathrm{~m})$ using a Juday-Bogorov net (1966-2003, $330 \mu \mathrm{m}$ mesh). Taxa abundance was counted from ongoing and historical samples using the wet-bed image scanning technique of ZooScan ${ }^{77}$. Sampling takes place on a seasonal basis and the PCA was performed at a seasonal scale using 6 zooplankton group for the 30- 
year period $1974-2003^{71}$. The first two PCs were retained and explained $30.53 \%$ and $13.86 \%$ of the variance, respectively. For this work we have used monthly abundances (ind $/ \mathrm{m}^{3}$ ) of the taxa Copepoda, Chaetognatha, Decapoda, Jellyfish, Siphonophora, plus Total Zooplankton Biovolume $\left(\mathrm{mm}^{3} / \mathrm{m}^{3}\right)$ from January 1974 to December 2003. This series has no major gaps (just a few months are missing).

4. The Northern Adriatic Sea, Eastern Mediterranean Sea (zooplankton). The Gulf of Trieste is the shallowest $(<27 \mathrm{~m})$, landlocked, northernmost section of the Adriatic Sea with a surface area of about $600 \mathrm{~km}^{2}$, and a volume of $9.5 \mathrm{~km}^{3} 78$. It is characterised by an overall shallowness $10 \%$ of the average bottom depth is less than $10 \mathrm{~m}$ and the maximum depth is about $23 \mathrm{~m}$ in the southern part; and by large and variable freshwater inputs ${ }^{34}$. The copepod community in the Gulf of Trieste is characterised by a few coastal and estuarine species, which in turn can exhibit high dominance. Sampling takes place on a monthly basis and the PCA was performed at a seasonal scale using 12 zooplankton groups or species for the 36-year period 1970-2005 ${ }^{71}$. The first two principal components (PCs) were retained and explained $17.70 \%$ and $12.63 \%$ of the variance, respectively. The copepod multidecadal time series includes the following taxa: Total copepods, Acartia clausi, Paracalanus parvus, Oithona spp, Oncaea spp, Centropages typicus, Pseudocalanus elongatus, Corycaeus spp, Temora longicornis, Centropages spp, Calanus helgolandicus, Temora stylifera, Euterpina acutifrons, Centropages kroyeri, Harpacticoida, Ctenocalanus vanus, Clausocalanus spp, and other copepods (see Conversi et al., 2009).

5. Western Atlantic - NOAA EcoMon - northern subarea (zooplankton abundance). The Ecosystem Monitoring (EcoMon) zooplankton sampling is carried out at about 120 stations, 6 times a year, over an area from Cape Hatteras, North Carolina, to Nova Scotia. Zooplankton samples were collected with a $61-\mathrm{cm}$ Bongo frame fitted with a net of $0.333 \mathrm{~mm}$ mesh, towed obliquely to a maximum depth of $200 \mathrm{~m}$, or $5 \mathrm{~m}$ from the bottom and back to the surface. Zooplankton are sorted, counted, and identified to the lowest possible taxa. Abundance is expressed as number per $100 \mathrm{~m}^{3}$ 79. Over this region, ecosystems are very distinct; the northern regions are dominated by Calanus finmarchicus whereas the species is only occasionally detected in the southern areas. For this reason, the area was divided into two subareas in Beaugrand and colleagues ${ }^{71}$ : a northern area which includes the Gulf of Maine and Georges Bank and a southern one, which includes the New England and Mid-Atlantic regions. Only the stations with a depth $>50 \mathrm{~m}$ (i.e. offshore) were considered. The northern area contained approximately 60 stations, sampled about 6 times per year, and the PCA was performed at a bi-monthly scale using 24 zooplankton groups or species for the 35-year period 1977-2011 $1^{71}$. The first two PCs were retained and explained $15.01 \%$ and $10.57 \%$ of the variance, respectively. The following taxa were considered in the analyses for the northern area: Centropages typicus, Calanus finmarchicus, Pseudocalanus spp., Penilia spp., Temora longicornis, Centropages hamatus, Echinodermata, Paracalanus parvus, Gastropoda, Acartia spp., Metridia lucens, Evadne spp., Oithona spp., Cirripedia, Hyperiidea, Gammaridea, Evadne nordmanni, Coelenterata, Copepoda, Clausocalanus arcuicornis, Decapoda, Protozoa, Polychaeta, Pisces.

6. Western Atlantic - NOAA EcoMon - southern subarea (zooplankton abundance). The southern subarea included the New England and Mid-Atlantic regions. Here also, only the stations with a water depth $>50 \mathrm{~m}$ (i.e. offshore) were taken into account. The southern subarea also contained approximately 60 stations, sampled about 6 times per year. The PCA was performed at a bi-monthly scale using 17 zooplanktonic groups or species for the 35-year period 1977$2011^{71}$. The first two PCs were retained and explained $19.31 \%$ and $11.07 \%$ of the variance, respectively. The following taxa were considered for the southern area: Calanus finmarchicus, 
Pseudocalanus spp., Penilia spp., Temora longicornis, Centropages hamatus, Paracalanus parvus, Acartia spp., Metridia lucens, Evadne spp., Oithona spp., Hyperiidea, Gammaridea, Evadne nordmanni, Copepoda, Clausocalanus arcuicornis, Protozoa, Pelecypoda.

7. Eastern Pacific, California Current (zooplankton biomass). The California Cooperative Oceanic Fisheries Investigations (CalCOFI) data set used in Beaugrand and colleagues ${ }^{71}$ was based on total zooplankton biomass displacement volume $\left(\mathrm{ml} / 1000 \mathrm{~m}^{3}\right)$ averaged across 55 stations consistently sampled from 1951 to 2009 (59 years) ${ }^{80}$. The data were averaged over time by season. During the period 1970-1977 sampling was carried out every three years (missing years: $1970,71,73,74,76,77)$. In addition, there are several missing seasons over the years. Therefore, seasonal data over the period 1951-2009 were corrected for changes in the sampling procedure in 1969 and 1977 by applying the methods of Ohman and Smith ${ }^{81}$. The PCA was performed at a seasonal scale (four seasons) using only one descriptor (zooplankton biomass displacement volume), although considering the 55 sampling stations, during the period $1951-2009^{71}$. The first two PCs were retained and explained $64.98 \%$ and $21.08 \%$ of the variance, respectively. For this work we use the seasonal data over the period 1951-2009, corrected for changes in the sampling procedure (in 1969 and 1977) using the methods of Ohman and Smith ${ }^{81}$.

8. Pacific Rim - north-eastern Pacific (wild salmon abundance). Total annual abundance (catch plus escapement) of "wild" pink salmon, chum salmon, and sockeye salmon population groups returning to 12 regions of Asia and North America were used for the 54-year time period 19522005 (annual data). Ruggerone and colleagues ${ }^{82}$ compiled all available annual data of wild adults of these species from South Korea, Japan, Russia, Alaska, British Columbia, and Washington (including the Columbia River). In Beaugrand and colleagues ${ }^{4}$, data were subdivided into two main areas (eastern Pacific and western Pacific) on the basis of the location of regional salmon stock groups ${ }^{82}$ and their main oceanic distribution ${ }^{83}$. Those areas include both neritic and oceanic regions. The PCA was calculated spatially at an annual scale over northeastern Pacific using abundance of 3 "wild" salmon species in 8 areas for 1952$2005^{71}$. The first two PCs were retained and explained $30.31 \%$ and $13.04 \%$ of the variance, respectively.

9. Pacific Rim - north-western Pacific (wild salmon abundance). In this area, the PCA was calculated spatially at an annual scale using abundance of 3 "wild" salmon species in 4 areas for $1952-2005$ (54 years) ${ }^{71}$. The first two PCs were retained and explained $27.77 \%$ and $20.63 \%$ of the variance, respectively.

10. Western Pacific, Oyashio Current (zooplankton abundance). The ODATE collection consists of $>18,000$ zooplankton samples collected in waters adjacent to Japan by several research institutes since the $1950 \mathrm{~s}$ and assembled together by Dr K. Odate. The ODATE sampling area covers both subarctic Oyashio and southern Transition waters, $35-43^{\circ} \mathrm{N}, 142-150^{\circ} \mathrm{E}$, which have distinctive water mass structure and zooplankton community structure. As the boundary of Oyashio and Transition water varies year by year, usually the water mass structure is defined by the water temperature criteria at $100 \mathrm{~m}$ depth (Oyashio: $\left\langle 5^{\circ} \mathrm{C}\right.$ ) and the zooplankton community is studied within the Oyashio (or Transition) water rather than a fixed latitudelongitude boundary. The ODATE Collection is not gathered via routine observation efforts, hence the time and location of sampling and the spatio-temporal resolution of data differ year by year ${ }^{84}$, and several months are missing throughout the time series. In this area, the PCA was calculated at a seasonal scale ( 2 seasonal periods: months 4-6 and 7-9) using 32 zooplankton species for 1960-2002 (43 years). Year 1977 was not considered in the study of Beaugrand and colleagues $^{71}$ because this year had abnormal values. The first two PCs were retained and 
explained $17.32 \%$ and $11.64 \%$ of the variance, respectively. In this work we used the monthly abundances (ind $/ 1000 \mathrm{~m}^{3}$ ) of the following species (April 1960-September 2002): Acartia longiremis, Acartia omorii, Calanus pacificus s.l., Candacia columbiae, Clausocalanus arcuicornis, Clausocalanus parapergens, Clausocalanus pergens, Corycaeus affinis, Ctenocalanus vanus, Eucalanus bungii, Gaetanus armiger, Lucicutia flavicornis, Mesocalanus tenuicornis, Metridia lucens, Metridia okhotensis, Metridia pacifica, Neocalanus cristatus, Neocalanus flemingeri, Neocalanus flemingeri and/or plumchrus, Neocalanus plumchrus, Oithona atlantica, Oithona similis, Oncaea borealis, Oncaea mediterranea, Oncaea venusta, Paracalanus parvus, Paraeuchaeta elongata, Pleuromamma scutullata, Pseudocalanus minutus, Pseudocalanus newmani, Racovitzanus antarcticus, Scolecithricella minor.

11. Western Pacific, Transition zone - south of the Oyashio Current (zooplankton abundance). In this area, the PCA was calculated at a seasonal scale ( 2 seasonal periods: months 4-6 and 7-9) using 67 species for 1960-2000 (41 years). Year 1977 was not considered in the study of Beaugrand and colleagues ${ }^{71}$ because this year had abnormal values. The first two PCs were retained and explained $15.26 \%$ and $13.76 \%$ of the variance, respectively. We used the monthly abundances (ind $/ 1000 \mathrm{~m}^{3}$ ) of the following species (April 1960-July 1999): Acartia danae, Acartia negligens, Acartia omorii, Calanus jashnovi, Calanus pacificus, Calocalanus pavo, Calocalanus plumulosus, Calocalanus styliremis, Calocalanus tenuis, Candacia bipinnata, Centropages bradyi, Clausocalanus arcuicornis, Clausocalanus farrani, Clausocalanus furcatus, Clausocalanus lividus, Clausocalanus mastigophorus, Clausocalanus minor, Clausocalanus parapergens, Clausocalanus pergens, Corycaeus affinis, Corycaeus crassiusculus, Corycaeus flaccus, Corycaeus furcifer, Corycaeus giesbrechti, Corycaeus pacificus, Corycaeus speciosus, Cosmocalanus darwini, Ctenocalanus vanus, Eucalanus bungii, Eucalanus californicus, Eucalanus hyalinus, Eucalanus subtenuis, Heterorhabdus papilliger, Lucicutia flavicornis, Mecynocera clausi, Mesocalanus tenuicornis, Metridia okhotensis, Metridia pacifica, Nanocalanus minor, Neocalanus cristatus, Neocalanus flemingeri, Neocalanus plumchrus, Oithona atlantica, Oithona longispina, Oithona plumifera, Oithona setigera, Oithona similis, Oncaea conifera, Oncaea mediterranea, Oncaea scottodicarloi, Oncaea venusta, Paracalanus aculeatus, Paracalanus parvus, Paraeuchaeta elongata, Pleuromamma abdominalis, Pleuromamma gracilis, Pleuromamma piseki, Pleuromamma xiphias, Pseudocalanus minutus, Pseudocalanus newmani, Sapphirina nigromaculata, Scaphocalanus curtus sensu Tanaka, Scaphocalanus echinatus, Scolecithricella dentata, Scolecithricella minor, Scolecithrix danae, Temora dicaudata.

A full description of these 11 regions, their species composition and biological data availability is presented in Beaugrand and colleagues ${ }^{71}$ (Supplementary Information). Three more datasets were added in this study.

12. San Francisco Bay (fish and crustaceans abundance) ${ }^{72}$. Cloern and colleagues ${ }^{72}$ analysed by PCA the catch data from monthly (February-October) bottom trawls taken by the California Department of Fish and Game at 24 sites in San Francisco Bay during the 29-year period 19802008. The PCA was performed on annual indices of abundance of 11 fish, 3 crabs and 4 caridean shrimps for the period 1980-2008. More details of the data are available in Cloern and colleagues ${ }^{72}$. In the present study, the analysis was updated to 2013 considering the matrix 35 years $x 11$ biological variables. The first two principal components, which explained $29.60 \%$ and $14.80 \%$ of the variance, respectively, were considered to be a good summary of the longterm community shift.

13. Hawaiian Ocean Time-series (zooplankton biomass) ${ }^{73}$. We used the database HOT (Hawaiian Ocean Time-series) from the Joint Global Ocean Flux Study which is representative of the North 
Pacific subtropical gyre. Monthly biomass data (in mg carbon (C) $\mathrm{m}^{-2}$ ) from 1994 onwards were collected at Station ALOHA (A Long-Term Oligotrophic Habitat Assessment; $22^{\circ} 45^{\prime} \mathrm{N}, 158^{\circ}$ $00^{\prime} \mathrm{W}$ ) located $100 \mathrm{~km}$ north of Oahu, Hawaii. For data collection, the net was towed obliquely from the surface to approximatively $175 \mathrm{~m}$ depth. The tows were then size fractioned through nested filters of the following mesh sizes: 5, 2 and $1 \mathrm{~mm}$ and $500 \mu \mathrm{m}$, and $200 \mu \mathrm{m}$. Here, the size fraction of $5 \mathrm{~mm}$ was not used because of methodological issues. More information on the HOT programme can be found at http://hahana.soest.hawaii.edu. We used zooplankton wet weight for four size fractions ( $2 \mathrm{~mm}, 1 \mathrm{~mm}, 500 \mu \mathrm{m}$, and $200 \mu \mathrm{m})$ and averaged for every two months for the 21-year period 1994-2014. A standardised PCA was applied on the matrix 21 years $x$ [ 4 size fractions $x 6$ months]. The first two principal components were retained and explained $43.39 \%$ and $14.55 \%$ of the variance, respectively.

14. Southern Ocean (zooplankton abundance) ${ }^{74}$. We used KRILLBASE, a circumpolar database of Antarctic krill and salps in the Southern Ocean https://www.bas.ac.uk/project/krillbase/. KRILLBASE has compiled all available scientific net-haul data into a single dataset, first published over a decade ago ${ }^{74}$ but recently updated. This project so far encompasses $\sim 15000$ net hauls for abundance of salps (all species and individuals of both solitary and aggregate forms combined) and postlarval krill, Euphausia superba). The database is derived from untargeted oblique or vertical net sampling data and spans the austral summers of 1926-1939 and 1976-2016. This is not a dataset collected by a single organised monitoring programme, but is a composite of all available net sample data collected on krill and salps. This includes rescued historical data as well as ongoing live monitoring programmes and pools data from 10 countries. The database is circumpolar and best coverage is from December to March in the Atlantic and Indian sectors (1926-1939, post-1976). The dataset, and the supporting data paper providing metadata, are available from the above website ${ }^{85}$. KRILLBASE needs careful analysis because the methods were not standardised. This is a particular problem for the actively swimming krill so the data used were standardised to a single, relatively efficient net sampling method using the method in Atkinson and colleagues ${ }^{86}$. We examined spatial and temporal changes in sampling and chose an area ranging from $66^{\circ} \mathrm{W}$ and $40^{\circ} \mathrm{W}$ of longitude, $64^{\circ} \mathrm{S}$ and $56^{\circ} \mathrm{S}$ of latitude (i.e. Antarctic Peninsula and western Scotia Sea areas) and a depth ranging from 0 to $400 \mathrm{~m}$. Other regions did not have sufficient information for the purpose of our study. We had to restrict our analysis to the 29-year time period 1975-2003 for months ranging from November to February to increase the percentage of data in the time series. A standardised PCA was applied on the matrix 29 years $\times$ [ 2 zooplankton taxa (Euphausia superba and salps)]. Note however that the standardised PCA performed in this case was to ensure homogeneity among analyses as only two variables were available. The first two PCs were retained and explained $54.80 \%$ and $45.20 \%$ of the variance, respectively.

\section{Supplementary Note 3| The MacroEcological Theory on the Arrangement of Life (METAL)}

Although Grinnell ${ }^{87}$, Elton ${ }^{88}$, Hutchinson ${ }^{89}$, Whittaker ${ }^{90}$ and more recently Chase and Leibold ${ }^{91}$ developed or used the concept of the niche, they did not explicitly connect this concept to the arrangement of life from the species to the community level.

At the species level, it is well-known that the niche/environment interaction enables the species' spatial distribution to be estimated with confidence at a macro-scale, explaining the strong development of Ecological Niche (ENM), Spatial Distribution (SDM) and macro-physiological Models ${ }^{92-}$ ${ }^{97}$. Indeed, Brown's theory ${ }^{98}$ states that species' local density and range are the result of the species' ecological niche. Far less known, however, is the fact that phenology and local year-to-year changes in species abundance, and their interaction within the spatial range of a species, can also be inferred from the knowledge of the niche ${ }^{99-101}$. At the species level, METAL enables the understanding and 
prediction of annual abundance, phenology and biogeography ${ }^{24,101-103}$. METAL has also been applied recently to explain the apparent inconsistency in the relationships between climate and species on a year-to-year basis ${ }^{99,100}$. Predictions of the effects of climate change on species are possible when the niche is assessed from physiology (fundamental niche) or spatial distribution (realised niche). The METAL framework not only explains the spatial and temporal responses of species to climatic variability or climate change but also enables these apparently independent responses to be connected throughout a theoretical framework ${ }^{24,102}$.

At a higher organisational level, the METAL theory may be used to better understand how communities organise themselves and how they may be affected by environmental changes ${ }^{25}$. Although the METAL theory has been tested for a limited number of species at present ${ }^{24,99-101}$, the evidence available so far suggests that the ecological niche of each species influences their spatial and temporal responses to climate change. If we admit this postulate, we can create a pool of pseudo-species, each having a unique niche after the principle of competitive exclusion of Gause ${ }^{104}$, and with the possibility of niche overlapping. Species are allowed to colonise a given oceanic region so long as they can survive changes in the environmental regime at different temporal scales. By reconstructing pseudo-communities, we can then investigate the origin of various ecogeographic patterns (e.g. latitudinal gradients in biodiversity, Rapoport's effect, Infrequency Law) ${ }^{24,105}$, community processes such as seasonal succession, properties of communities and their consequences for ecosystem functioning, regulating and provisioning services ${ }^{16,103,105}$. METAL shows that large-scale biodiversity patterns are influenced by an underlying mathematical constraint, we called the chessboard of $l i f e^{26}$, that results from the interaction between the ecological niche of a species and spatial and temporal fluctuations in the environment.

Recently, we have theoretically investigated palaeo (mid-Pliocene and Last Glacial Maximum or LGM), contemporaneous (1960-2013) and future (2080-2100) changes in biodiversity in the context of global climate change to evaluate the sensitivity and vulnerability of biodiversity to climate change ${ }^{103}$. This theoretical work has revealed that climate change may rapidly alter marine biodiversity over large oceanic regions and that the intensity of this reorganisation will depend on the magnitude of warming. If global warming is small (RCP2.6), the study has shown that biological changes would reflect $15.5 \%$ of the amount of change seen between the LGM and the present day, or $25.3 \%$ of the amount of change observed between the mid-Pliocene and today; neither are much different from annual variability (1960-2013) and so it may be benign overall. If warming is moderate (RCP4.5), changes in marine biodiversity will be three-times more extensive and at least twice as strong in magnitude than changes observed over the last 50 years. If global warming is severe (RCP6.0 and 8.5), between 50 and $70 \%$ of the global ocean will experience a change in marine biodiversity equivalent to, or higher than, that experienced between the LGM/mid-Pliocene and today, emphasizing that climate warming will have a major effect on marine biodiversity.

In the previous work, we did not investigate decadal changes at the community level nor did we focus on Abrupt Community Shifts (ACSs). Recently, we have designed another METAL model to specifically investigate long-term changes in community, including $\mathrm{ACSs}^{16}$. The full procedure used in the present article is described in detail in Beaugrand ${ }^{16}$ and was tested in the North Sea using Continuous Plankton Recorder (CPR) data ${ }^{16}$. Here, we use time series originating from 14 marine pelagic ecosystems in tropical, temperate and polar regions to test extensively the method and apply it at a global scale to investigate spatial and temporal patterns as well as processes at the origin of climate-induced ACSs in the pelagic ocean.

\section{Supplementary Note 4| Test of the abrupt shift detection algorithm}


We tested our procedure using order-1-5 weighted differences in 5 different situations (Supplementary Figures 8-12). Simulated time series used to test our procedure had different degrees of autocorrelation (Supplementary Figure 13). Expectedly, autocorrelation was reinforced by the use of the first-order symmetrical moving average. In Supplementary Figures 8-12, we combined the results of the first to fifth weighted differences. In the first situation, we generated randomly a time series of 100 years, with maximum amplitude of 1 (Supplementary Figure 8). One value crossed the threshold of 3 (Supplementary Figure 8b) but when an order-1 moving average was applied prior to the analysis, no value reached the threshold (Supplementary Figure $8 \mathrm{c}$ ). In the second situation, we generated a linear trend of amplitude $=0.5$ in addition to white noise with a maximum amplitude of 1 (Supplementary Figure 9). A result close to the previous situation was observed. This example shows that our procedure is unlikely affected by temporal autocorrelation. In the third situation, we generated a cycle with amplitude of 1 in addition to the linear trend and white noise (Supplementary Figure 10). Significant abrupt shifts were detected, often corresponding to acceleration phases between the minimum and the maximum of a cycle and resulting from the interaction between white noise and the cycle (Supplementary Figure 10b). After diminishing the white noise by using the order1 moving average, no significant abrupt shift was detected (Supplementary Figure 10c). This result reflects an important property of our algorithm: it is sensitive to the variability throughout the time series. When the variability throughout the time series is high, this makes more difficult the detection of an abrupt shift. Therefore, our algorithm considers the inner variability of the time series to identify a significant shift ${ }^{60}$. In the fourth situation, we added an abrupt shift of amplitude 1 (Supplementary Figure 11). The shift was detected for all order-1-5 differences. The application of an order-1 moving average improved detection by diminishing false detection. In the fifth situation, we added an episodic event of amplitude 1 (Supplementary Figure 12). The algorithm identified the abrupt shift as well as the beginning and the end of the episodic event, results being clearer when an order-1 moving average was applied prior to the analysis. It should be noted that there can be a big difference in the index of abruptness, simply resulting from the interaction between the signal and white noise. White noise can therefore strongly alter our perception of a shift, a result already suggested in some studies, which explains why we used an order-1 symmetrical moving average prior to the analysis ${ }^{56}$. In our study, we focused on abrupt shifts and not on changes related to the passage of a phase to another within a cycle or pseudo-cycle. Therefore, community shifts related to pseudo-cyclical variability ${ }^{106}$ may not be revealed in this study. 


\section{Supplementary Tables}

Supplementary Table 1 | List of the abbreviations used in this study.

ACS

ASD

HOT

METAL

OBS-PC

PCA

PC

Pred-PC

SLP

SST
Abrupt Community Shift

Abrupt Shift Detection algorithm

Hawaiian Ocean Time-series

Macro-Ecological Theory on the

Arrangement of Life

Principal Component based on observed

biological data

Principal Component Analysis

Principal Component

Principal Component based on simulated data (pseudo-species)

Sea Level Pressure

Sea Surface Temperature 
Supplementary Table 2 | Main characteristics of observed biological time series for the 14 study systems.

\begin{tabular}{|c|c|c|c|c|c|c|}
\hline Area & Period & Frequency & Gaps & Source & Variables & Notes \\
\hline $\begin{array}{l}\text { 1. Central } \\
\text { North Sea }\end{array}$ & 1958- 2007 & monthly & no & $\begin{array}{l}\text { SAHFOS } \\
\text { http://www.sahfo } \\
\text { s.ac.uk }\end{array}$ & zooplankton & CPR \\
\hline $\begin{array}{l}\text { 2. Central } \\
\text { Baltic Sea }\end{array}$ & $1959-2008$ & seasonal & no & Otto & zooplankton & Juday Net \\
\hline 3. Ligurian Sea & $1974-2003$ & monthly & no & $\begin{array}{l}\text { Laboratoire } \\
\text { d'Océanologie de } \\
\text { Villefranche } \\
\text { http://www.obs- } \\
\underline{\text { vlfr.fr/ }}\end{array}$ & zooplankton & $\begin{array}{ll}\text { Juday, } & \text { Bogorov } \\
\text { net, } & \text { Zooscan } \\
\text { imaging } & \\
\text { technique } & \end{array}$ \\
\hline $\begin{array}{lr}4 . \quad \text { Northern } \\
\text { Adriatic Sea } \\
\text { (Gulf } r \text { of } \\
\text { Trieste) }\end{array}$ & 1970- 2005 & monthly & $\begin{array}{l}\text { Yes } \\
(1981- \\
1985)\end{array}$ & Conversi & zooplankton & WP2 net \\
\hline $\begin{array}{l}\text { 5. Northwest } \\
\text { Atlantic } \\
\text { Northern area }\end{array}$ & 1977- 2011 & seasonal & no & $\begin{array}{l}\frac{\text { http://osprey.bco }}{\text { dmo.org/dataset. }} \\
\text { cfm?flag=view\&id } \\
=13684\end{array}$ & zooplankton & $\begin{array}{l}\text { Marmap bongo } \\
\text { data }\end{array}$ \\
\hline $\begin{array}{l}\text { 6. Northwest } \\
\text { Atlantic } \\
\text { Southern area }\end{array}$ & 1977- 2011 & seasonal & no & $\begin{array}{l}\frac{\text { http://osprey.bco }}{\text { dmo.org/dataset. }} \\
\text { cfm?flag=view\&id } \\
=13684\end{array}$ & zooplankton & $\begin{array}{l}\text { Marmap bongo } \\
\text { data }\end{array}$ \\
\hline $\begin{array}{l}\text { 7. California } \\
\text { Current }\end{array}$ & 1951-2009 & seasonal & no & $\begin{array}{l}\text { California } \\
\text { Cooperative } \\
\text { Oceanic Fisheries } \\
\text { Investigations: } \\
\text { http://data.calcof } \\
\text { i.org/zooplankton } \\
. \text { html }\end{array}$ & zooplankton & CAICOFI \\
\hline $\begin{array}{l}\text { 8. E. Pacific } \\
\text { Rim }\end{array}$ & 1952- 2005 & annual & no & $\frac{\text { http://hdl.handle. }}{\frac{\text { net/1773/16262 }}{107}}$ & wild salmon & Large region \\
\hline
\end{tabular}




\begin{tabular}{|c|c|c|c|c|c|c|}
\hline Area & Period & Frequency & Gaps & Source & Variables & Notes \\
\hline $\begin{array}{l}\text { 9. W. Pacific } \\
\text { Rim }\end{array}$ & $1952-2005$ & annual & no & $\frac{\text { http://hdl.handle. }}{\frac{\text { net/1773/16262 }}{107}}$ & wild salmon & Large region \\
\hline $\begin{array}{l}\text { 10. Oyashio } \\
\text { Current }\end{array}$ & 1960-2002 & $\begin{array}{l}\text { Seasonal (April- } \\
\text { June and July- } \\
\text { September) }\end{array}$ & Yes (1969) & $\begin{array}{l}\text { Sugisaki, Chiba } \\
\text { http://tnfri.fra.aff } \\
\underline{\text { rc.go.jp/eindex.ht }} \\
\underline{\mathrm{ml}}\end{array}$ & zooplankton & NORPAC ring net \\
\hline $\begin{array}{l}\text { 11. Trans } \\
\text { ition zone } \\
\text { (south of the } \\
\text { Oyashio } \\
\text { Current) }\end{array}$ & $1960-2000$ & $\begin{array}{l}\text { Seasonal } \\
\text { (April-June and } \\
\text { July-September) }\end{array}$ & Yes (1992) & $\begin{array}{l}\text { Sugisaki, Chiba } \\
\text { http://tnfri.fra.aff } \\
\underline{\text { rc.go.jp/eindex.ht }} \\
\underline{\mathrm{ml}}\end{array}$ & zooplankton & NORPAC ring net \\
\hline 12. НОТ & $1994-2014$ & Monthly & $\begin{array}{l}\text { Yes (some } \\
\text { months) }\end{array}$ & $\begin{array}{l}\text { http://hahana.so } \\
\text { est.hawaii.edu/ho } \\
\text { t/methods/plankt } \\
\text { on.html }\end{array}$ & zooplankton & 4 size fractions \\
\hline $\begin{array}{l}\text { 13. San } \\
\text { Francisco Bay }\end{array}$ & $1980-2013$ & Annual mean & No & Cloern & $\begin{array}{l}\text { decapods and } \\
\text { fish }\end{array}$ & \\
\hline $\begin{array}{l}\text { 14. Antar } \\
\text { ctic Peninsula } \\
\text { Area }\end{array}$ & $1975-2003$ & Seasonal & $\begin{array}{l}\text { Yes (1979, } \\
1982, \\
1986, \\
1992)\end{array}$ & $\begin{array}{l}\frac{\text { https://www.bas. }}{\text { ac.uk/project//krill }} \\
\underline{\text { base/ }}\end{array}$ & zooplankton & $\begin{array}{l}\text { Euphausiids and } \\
\text { salps }\end{array}$ \\
\hline
\end{tabular}


Supplementary Table 3 | Main characteristics of the 14 observed and simulated matrices analysed by standardised PCA. When total zooplankton biomass (California Current) or size fractions (HOT) were used, we chose arbitrarily pseudo-communities composed of 30 pseudospecies. The selection of the number of pseudo-species did not affect significantly the results above 5 pseudo-species. The last column also contains the area (expected as million (M) $\mathrm{km}^{2}$ ) of each region used to test our procedure and its average bathymetry $(\mathrm{m})$. Note that this is not the area where sampling took place but rather the area chosen to test our model.

\begin{tabular}{|c|c|c|c|c|}
\hline Area & $\begin{array}{c}\text { Biological } \\
\text { parameters in } \\
\text { observed matrices }\end{array}$ & $\begin{array}{c}\text { Area covered by } \\
\text { observed } \\
\text { community }\end{array}$ & $\begin{array}{l}\text { Biological } \\
\text { parameters in } \\
\text { theorized } \\
\text { matrices }\end{array}$ & $\begin{array}{l}\text { Area considered to } \\
\text { reconstruct pseudo- } \\
\text { community }\end{array}$ \\
\hline 1. Central North Sea & $\begin{array}{l}14 \text { species or } \\
\text { taxonomic groups }\end{array}$ & $\begin{array}{l}3^{\circ} \mathrm{W}-10^{\circ} \mathrm{E} \\
51-60^{\circ} \mathrm{N}\end{array}$ & $\begin{array}{l}14 \text { pseudo- } \\
\text { species }\end{array}$ & $\begin{array}{l}3^{\circ} \mathrm{W}-10^{\circ} \mathrm{E} \\
51-60^{\circ} \mathrm{N} \\
\text { Bathymetry: } 81 \mathrm{~m} \\
\text { Surface area: } 0.85 \mathrm{M} \mathrm{km}^{2}\end{array}$ \\
\hline 2. Central Baltic Sea & $\begin{array}{l}10 \text { species or } \\
\text { taxonomic groups } x \\
4 \text { 3-month periods }\end{array}$ & $\begin{array}{l}19^{\circ} 10^{\prime}-22^{\circ} 10^{\prime} \mathrm{E} \\
56.30^{\prime}-58^{\circ} 30^{\prime} \mathrm{N}\end{array}$ & $\begin{array}{l}10 \text { pseudo- } \\
\text { species } \times 43- \\
\text { month periods }\end{array}$ & $\begin{array}{l}18-22^{\circ} \mathrm{E} \\
56-58^{\circ} \mathrm{N} \\
\text { Bathymetry: } 83 \mathrm{~m} \\
\text { Surface area: } 0.06 \mathrm{M} \mathrm{km}^{2}\end{array}$ \\
\hline Ligurian Sea & $\begin{array}{l}6 \text { zooplankton } \\
\text { groups x } 4 \text { 3-month } \\
\text { periods }\end{array}$ & $\begin{array}{l}07^{\circ} 19 \mathrm{E} \\
43^{\circ} 41 \mathrm{~N}\end{array}$ & $\begin{array}{l}6 \text { pseudo-species } \\
\times 43 \text {-month } \\
\text { periods }\end{array}$ & $\begin{array}{l}6-8^{\circ} \mathrm{E} \\
42-44^{\circ} \mathrm{N} \\
\text { Bathymetry: } 2283 \mathrm{~m} \\
\text { Surface area: } 0.04 \mathrm{M} \mathrm{km}^{2}\end{array}$ \\
\hline $\begin{array}{l}\text { 4. Northern Adriatic } \\
\text { Sea (Gulf of Trieste) }\end{array}$ & $\begin{array}{l}12 \text { species or } \\
\text { taxonomic groups } x \\
4 \text { 3-month periods }\end{array}$ & $\begin{array}{l}13^{\circ} 42^{\prime} 36^{\prime \prime} \mathrm{E} \\
45^{\circ} 42^{\prime} 03^{\prime \prime} \mathrm{N}\end{array}$ & $\begin{array}{l}12 \text { pseudo- } \\
\text { species } \times 43- \\
\text { month periods }\end{array}$ & $\begin{array}{l}12-14^{\circ} \mathrm{E} \\
42-46^{\circ} \mathrm{N} \\
\text { Bathymetry: } 31 \mathrm{~m} \\
\text { Surface area: } 0.08 \mathrm{M} \mathrm{km}^{2}\end{array}$ \\
\hline $\begin{array}{l}5 . \quad \text { Northwest } \\
\text { Atlantic } \\
\text { Northern area }\end{array}$ & $\begin{array}{l}24 \text { species or } \\
\text { taxonomic groups } x \\
62 \text {-month periods }\end{array}$ & $\begin{array}{l}65.17-70.95^{\circ} \mathrm{W} \\
40.09-44.83^{\circ} \mathrm{N}\end{array}$ & $\begin{array}{l}24 \text { pseudo- } \\
\text { species } \times 62 \text { - } \\
\text { month periods }\end{array}$ & $\begin{array}{l}65-70^{\circ} \mathrm{W} \\
41-44^{\circ} \mathrm{N} \\
\text { Bathymetry: } 317 \mathrm{~m} \\
\text { Surface area: } 0.13 \mathrm{M} \mathrm{km}^{2}\end{array}$ \\
\hline $\begin{array}{l}6 . \quad \text { Northwest } \\
\text { Atlantic } \\
\text { Southern area }\end{array}$ & $\begin{array}{l}17 \text { species or } \\
\text { taxonomic groups } x \\
62 \text {-month periods }\end{array}$ & $\begin{array}{l}68.79-76.07^{\circ} \mathrm{W} \\
35.14-41.69^{\circ} \mathrm{N}\end{array}$ & $\begin{array}{l}17 \text { pseudo- } \\
\text { species } \times 62 \text { - } \\
\text { month periods }\end{array}$ & $\begin{array}{l}68-76^{\circ} \mathrm{W} \\
35-41^{\circ} \mathrm{N} \\
\text { Bathymetry: } 2363 \mathrm{~m} \\
\text { Surface area: } 0.47 \mathrm{M} \mathrm{km}^{2}\end{array}$ \\
\hline 7. California Current & $\begin{array}{l}1 \text { biological } \\
\text { attribute (total } \\
\text { zooplankton } \\
\text { biomass) x } 43 \text { - } \\
\text { month periods }\end{array}$ & $\begin{array}{l}117-124^{\circ} \mathrm{W} \\
31-35^{\circ} \mathrm{N}\end{array}$ & $\begin{array}{l}30 \text { pseudo- } \\
\text { species } \times 43- \\
\text { month periods }\end{array}$ & $\begin{array}{l}116-124^{\circ} \mathrm{W} \\
30-36^{\circ} \mathrm{N} \\
\text { Bathymetry: } 2768 \mathrm{~m} \\
\text { Surface area: } 0.48 \mathrm{M} \mathrm{km}^{2}\end{array}$ \\
\hline E. Pacific Rim & $\begin{array}{l}3 \text { species } \\
\text { abundance in } 8 \\
\text { areas }\end{array}$ & $\begin{array}{l}120-170^{\circ} \mathrm{W} \\
50-60^{\circ} \mathrm{N}\end{array}$ & $\begin{array}{l}24 \text { pseudo- } \\
\text { species }\end{array}$ & $\begin{array}{l}120-170^{\circ} \mathrm{W} \\
50-60^{\circ} \mathrm{N} \\
\text { Bathymetry: } 2612 \mathrm{~m} \\
\text { Surface area: } 3.53 \mathrm{M} \mathrm{km}^{2}\end{array}$ \\
\hline W. Pacific Rim & $\begin{array}{l}3 \text { species } \\
\text { abundance in } 4 \\
\text { areas }\end{array}$ & $\begin{array}{l}140-170^{\circ} \mathrm{E} \\
40-60^{\circ} \mathrm{N}\end{array}$ & $\begin{array}{l}12 \text { pseudo- } \\
\text { species }\end{array}$ & $\begin{array}{l}140-170^{\circ} \mathrm{E} \\
40-60^{\circ} \mathrm{N} \\
\text { Bathymetry: } 3044 \mathrm{~m} \\
\text { Surface area: } 4.73 \mathrm{M} \mathrm{km}^{2}\end{array}$ \\
\hline Oyashio Current & $\begin{array}{l}32 \text { species or } \\
\text { taxonomic groups } x \\
2 \text { periods (months } \\
4-6 \text { and } 7-9 \text { ) }\end{array}$ & $\begin{array}{l}142-149.29^{\circ} \mathrm{E} \\
37.36-42.96^{\circ} \mathrm{N}\end{array}$ & $\begin{array}{l}32 \text { pseudo- } \\
\text { species } \times 2 \\
\text { periods (months } \\
4-6 \text { and } 7-9 \text { ) }\end{array}$ & $\begin{array}{l}144-148^{\circ} \mathrm{E} \\
38-42^{\circ} \mathrm{N} \\
\text { Bathymetry: } 5359 \mathrm{~m} \\
\text { Surface area: } 0.16 \mathrm{M} \mathrm{km}^{2}\end{array}$ \\
\hline Transition zone & $\begin{array}{l}67 \text { species or } \\
\text { taxonomic groups } x\end{array}$ & $\begin{array}{l}144.95-150.02^{\circ} \mathrm{E} \\
35.18-42.53^{\circ} \mathrm{N}\end{array}$ & $\begin{array}{l}67 \text { pseudo- } \\
\text { species } \times 2\end{array}$ & $\begin{array}{l}144-150^{\circ} \mathrm{E} \\
36-42^{\circ} \mathrm{N}\end{array}$ \\
\hline
\end{tabular}




\begin{tabular}{|c|c|c|c|c|}
\hline $\begin{array}{l}\text { (south of the Oyashio } \\
\text { Current) }\end{array}$ & $\begin{array}{l}2 \text { periods (months } \\
4-6 \text { and } 7-9 \text { ) }\end{array}$ & & $\begin{array}{l}\text { periods (months } \\
4-6 \text { and } 7-9 \text { ) }\end{array}$ & $\begin{array}{l}\text { Bathymetry: } 5492 \mathrm{~m} \\
\text { Surface area: } 0.37 \mathrm{M} \mathrm{km}^{2}\end{array}$ \\
\hline HOT & $\begin{array}{l}4 \text { size fractions } \times 6 \\
2 \text {-month periods }\end{array}$ & $158^{\circ} 00^{\prime} \mathrm{W} 22^{\circ} 45^{\prime} \mathrm{N}$ & $\begin{array}{l}30 \text { pseudo- } \\
\text { species } \times 62 \text { - } \\
\text { month periods }\end{array}$ & $\begin{array}{l}158.5-157.5^{\circ} \mathrm{W} \\
22-23^{\circ} \mathrm{N} \\
\text { Bathymetry: } 4502 \mathrm{~m} \\
\text { Surface area: } 0.02 \mathrm{M} \mathrm{km}^{2}\end{array}$ \\
\hline $\begin{array}{l}\text { 2. San Francisco } \\
\text { Bay }\end{array}$ & $\begin{array}{l}11 \text { species or } \\
\text { taxonomic groups }\end{array}$ & $\begin{array}{l}\text { San Francisco Bay } \\
\left(121^{\prime} 45^{\prime \prime}-122^{\prime} 30^{\prime \prime}\right. \\
\mathrm{N} \text { and } 37^{\prime} 30^{\prime \prime}-38^{\prime} \\
\left.15^{\prime \prime} \mathrm{W}\right)\end{array}$ & $\begin{array}{l}11 \text { pseudo- } \\
\text { species }\end{array}$ & $\begin{array}{l}120-122^{\circ} \mathrm{W} \\
36-38^{\circ} \mathrm{N} \\
\text { Bathymetry: } 286 \mathrm{~m} \\
\text { Surface area: } 0.04\end{array}$ \\
\hline $\begin{array}{l}\text { 3. Antarctic } \\
\text { Peninsula Area }\end{array}$ & $\begin{array}{l}2 \text { zooplankton taxa } \\
\text { (Euphausia superba } \\
\text { and salps) x a 4- } \\
\text { month periods } \\
\text { (November to } \\
\text { February) }\end{array}$ & $\begin{array}{l}40-66^{\circ} \mathrm{W} \\
56-64^{\circ} \mathrm{S}\end{array}$ & $\begin{array}{l}2 \text { pseudo-species } \\
\times 2 \text {-month } \\
\text { periods } \\
\text { (November to } \\
\text { February) }\end{array}$ & $\begin{array}{l}40-66^{\circ} \mathrm{W} \\
56-64^{\circ} \mathrm{S} \\
\text { Bathymetry: } 2945 \mathrm{~m} \\
\text { Surface area: } 1.28\end{array}$ \\
\hline
\end{tabular}


Supplementary Table 4 | Results of the principal component analyses performed on biological variables in the 14 marine systems. Observed and predicted (broken-stick distribution) first two eigenvalues. Bold: significant relative eigenvalues.

\begin{tabular}{|c|c|c|c|c|c|}
\hline Area & $\begin{array}{l}\text { Number of } \\
\text { variables }\end{array}$ & $\begin{array}{l}\text { Observed } \\
\text { eigenvalue } 1\end{array}$ & $\begin{array}{l}\text { Observed } \\
\text { eigenvalue } 2\end{array}$ & $\begin{array}{l}\text { Predicted } \\
\text { Eigenvalue } 1\end{array}$ & $\begin{array}{l}\text { Predicted } \\
\text { Eigenvalue } 2\end{array}$ \\
\hline $\begin{array}{c}\text { Central North } \\
\text { Sea }\end{array}$ & 14 & 27.96 & 27.15 & 23.25 & 16.08 \\
\hline $\begin{array}{c}\text { Central Baltic } \\
\text { Sea }\end{array}$ & 40 & 23.08 & 11.12 & 10.67 & 8.19 \\
\hline Ligurian Sea & 20 & 30.53 & 13.86 & 17.97 & 12.98 \\
\hline $\begin{array}{c}\text { Northern } \\
\text { Adriatic Sea } \\
\text { (Gulf of } \\
\text { Trieste) }\end{array}$ & 48 & 17.70 & 12.63 & 9.30 & 7.21 \\
\hline $\begin{array}{c}\text { Northwest } \\
\text { Atlantic } \\
\text { Northern area }\end{array}$ & 144 & 15.01 & 10.57 & 3.85 & 3.16 \\
\hline $\begin{array}{c}\text { Northwest } \\
\text { Atlantic }\end{array}$ & 102 & 19.31 & 11.07 & 5.11 & 4.13 \\
\hline Southern area & & & & & \\
\hline $\begin{array}{l}\text { California } \\
\text { Current }\end{array}$ & 4 & 64.98 & 21.08 & 52.12 & 27.08 \\
\hline E. Pacific Rim & 24 & 30.31 & 13.04 & 15.73 & 11.58 \\
\hline W. Pacific Rim & 12 & 27.77 & 20.63 & 25.85 & 17.53 \\
\hline $\begin{array}{l}\text { Oyashio } \\
\text { Current }\end{array}$ & 64 & 17.32 & 11.64 & 7.41 & 5.85 \\
\hline $\begin{array}{l}\text { Transition } \\
\text { zone } \\
\text { (south of the } \\
\text { Oyashio } \\
\text { Current) }\end{array}$ & 134 & 15.26 & 13.76 & 4.08 & 3.34 \\
\hline НОТ & 24 & 43.39 & 14.55 & 15.75 & 11.56 \\
\hline $\begin{array}{c}\text { San Francisco } \\
\text { Bay }\end{array}$ & 11 & 29.60 & 14.80 & 27.42 & 18.35 \\
\hline $\begin{array}{c}\text { Antarctic } \\
\text { Peninsula } \\
\text { Area }\end{array}$ & 2 & 54.80 & 45.20 & 75 & 25 \\
\hline
\end{tabular}


Supplementary Table 5 | Absolute values of linear correlation coefficients calculated between the first and second principal components originating from a PCA performed on observed communities (Obs-PC1-2) and the average of the first and second PCs originating from theoretical communities (Pred-PC1-2). Values in bold were significant and higher than 0.47. Asterisks highlight couples of variables that were used in Figure 2.

\begin{tabular}{|c|c|c|c|c|}
\hline Areas & PredPC1/ObsPC1 & PredPC1/ObsPC2 & PredPC2/ObsPC1 & PredPC2/ObsPC2 \\
\hline $\begin{array}{c}\text { Central North } \\
\text { Sea }\end{array}$ & $0.84^{*}$ & 0.11 & 0.47 & 0.28 \\
\hline $\begin{array}{c}\text { Central Baltic } \\
\text { Sea }\end{array}$ & $0.86^{*}$ & 0.17 & 0.25 & 0.62 \\
\hline Ligurian Sea & 0.17 & 0.28 & $0.48 *$ & 0.61 \\
\hline $\begin{array}{l}\text { Northern } \\
\text { Adriatic Sea } \\
\text { (Gulf of } \\
\text { Trieste) }\end{array}$ & $0.87^{*}$ & 0.12 & 0.15 & 0.18 \\
\hline $\begin{array}{c}\text { Northwest } \\
\text { Atlantic } \\
\text { Northern area }\end{array}$ & $0.54^{*}$ & 0.32 & 0.18 & 0.29 \\
\hline $\begin{array}{c}\text { Northwest } \\
\text { Atlantic } \\
\text { Southern area }\end{array}$ & 0.14 & $0.49 *$ & 0.16 & 0.27 \\
\hline $\begin{array}{c}\text { California } \\
\text { Current }\end{array}$ & $0.55^{*}$ & 0.32 & 0.42 & 0.42 \\
\hline E. Pacific Rim & $0.67^{*}$ & 0.01 & 0.11 & 0.02 \\
\hline W. Pacific Rim & 0.09 & $0.67 *$ & 0.26 & 0.38 \\
\hline $\begin{array}{l}\text { Oyashio } \\
\text { Current }\end{array}$ & $0.69 *$ & 0.11 & 0.19 & 0.06 \\
\hline $\begin{array}{c}\text { Transition } \\
\text { zone } \\
\text { (south of the } \\
\text { Oyashio } \\
\text { Current) }\end{array}$ & $0.18^{*}$ & 0.22 & 0.08 & 0.29 \\
\hline HOT & 0.12 & 0.35 & $0.51 *$ & 0.13 \\
\hline $\begin{array}{c}\text { San Francisco } \\
\text { Bay }\end{array}$ & $0.79 *$ & 0.18 & 0.61 & 0.29 \\
\hline $\begin{array}{c}\text { Antarctic } \\
\text { Peninsula } \\
\text { Area }\end{array}$ & 0.25 & $0.67^{*}$ & 0.44 & 0.37 \\
\hline
\end{tabular}


Supplementary Table 6 | Correlations (normalized eigenvectors, EV) between long-term changes in the first three principal components ('global' Obs-PC1-3 and Pred-PC1-3) originating from a 'global' PCA performed on observed communities ( 14 Obs-PC1-2 $=28$ variables) and a 'global' PCA performed on pseudo-communities (14 Pred-PC1-2 $=28$ variables) and corresponding long-term changes in all first 2 Obs-PC1-2 and Pred-PC1-2, respectively. Values in bold are higher than 0.5. PC: Principal Component. EV: normalised eigenvectors that are correlations between each variable and the corresponding principal components (EV1-3: correlation with the first, second and third PC).

\begin{tabular}{|c|c|c|c|c|c|c|c|}
\hline \multirow[b]{2}{*}{ Ecoregions } & \multirow[b]{2}{*}{ PC } & \multicolumn{2}{|c|}{ PCA on observations } & \multicolumn{3}{|c|}{ PCA on predictions } & \multirow[b]{2}{*}{ EV 3} \\
\hline & & EV 1 & EV 2 & EV 3 & EV 1 & EV 2 & \\
\hline \multirow{2}{*}{ Baltic Sea } & PC 1 & 0.91 & -0.13 & -0.11 & 0.85 & -0.23 & -0.13 \\
\hline & PC 2 & -0.14 & -0.67 & 0.34 & 0.07 & -0.14 & 0.70 \\
\hline \multirow[t]{2}{*}{ Ligurian Sea } & PC 1 & -0.49 & -0.45 & -0.71 & 0.83 & -0.10 & 0.07 \\
\hline & PC 2 & -0.31 & 0.02 & -0.05 & 0.40 & 0.38 & -0.57 \\
\hline \multirow[t]{2}{*}{ CALCOFI } & PC 1 & -0.80 & -0.12 & 0.02 & -0.29 & -0.32 & -0.08 \\
\hline & PC 2 & 0.12 & -0.10 & 0.40 & -0.19 & 0.00 & 0.65 \\
\hline \multirow[t]{2}{*}{ Adriatic Sea } & PC 1 & 0.89 & -0.04 & -0.29 & -0.77 & 0.16 & -0.32 \\
\hline & PC 2 & -0.17 & 0.55 & -0.73 & 0.33 & 0.03 & -0.52 \\
\hline \multirow[t]{2}{*}{ Oyashio } & PC 1 & 0.59 & 0.43 & -0.36 & -0.65 & 0.31 & 0.02 \\
\hline & PC 2 & -0.02 & 0.62 & -0.80 & 0.39 & 0.67 & -0.37 \\
\hline \multirow[t]{2}{*}{ Transition } & PC 1 & 0.23 & 0.67 & -0.67 & -0.72 & 0.43 & 0.52 \\
\hline & PC 2 & -0.07 & -0.57 & 0.49 & 0.20 & 0.08 & -0.35 \\
\hline \multirow[t]{2}{*}{ East Pacific } & PC 1 & 0.48 & 0.49 & -0.79 & 0.38 & 0.31 & 0.50 \\
\hline & PC 2 & -0.73 & -0.25 & -0.23 & -0.30 & -0.11 & -0.18 \\
\hline \multirow[t]{2}{*}{ West Pacific } & PC 1 & 0.58 & -0.26 & -0.10 & 0.74 & 0.46 & -0.18 \\
\hline & PC 2 & 0.84 & 0.28 & 0.23 & 0.41 & 0.43 & 0.22 \\
\hline \multirow[t]{2}{*}{ Northwest Atlantic (North) } & PC 1 & 0.70 & -0.57 & 0.13 & 0.23 & -0.78 & 0.22 \\
\hline & PC 2 & 0.52 & 0.54 & 0.59 & 0.58 & 0.32 & 0.37 \\
\hline \multirow[t]{2}{*}{ Northwest Atlantic (South) } & PC 1 & -0.83 & -0.08 & -0.73 & 0.20 & -0.81 & 0.31 \\
\hline & PC 2 & -0.19 & 0.52 & 0.49 & -0.45 & -0.51 & -0.26 \\
\hline \multirow[t]{2}{*}{ North Sea } & PC 1 & -0.78 & 0.04 & 0.46 & -0.85 & 0.16 & -0.02 \\
\hline & PC 2 & 0.03 & -0.25 & 0.12 & 0.01 & 0.13 & -0.81 \\
\hline \multirow[t]{2}{*}{ San Francisco } & PC 1 & 0.52 & -0.72 & 0.10 & -0.07 & 0.84 & -0.21 \\
\hline & PC 2 & 0.73 & 0.29 & 0.30 & -0.33 & -0.03 & 0.65 \\
\hline \multirow[t]{2}{*}{ HOT } & PC 1 & -0.38 & -0.97 & -0.50 & -0.23 & 0.70 & 0.55 \\
\hline & PC 2 & -0.67 & -0.46 & -0.52 & 0.54 & -0.34 & 0.17 \\
\hline \multirow[t]{2}{*}{ Antarctic Peninsula Area } & PC 1 & -0.15 & -0.37 & 0.23 & 0.33 & 0.47 & -0.75 \\
\hline & PC 2 & 0.60 & 0.31 & 0.29 & 0.22 & 0.03 & 0.14 \\
\hline
\end{tabular}


Supplementary Table 7 | Linear correlations between the spatial extent of predicted abrupt community shifts from 1960 to 2015 (degree of freedom = 51) and the spatial extent of significant abrupt climatic shifts based on six climatic parameters. pACF: probability of significance with correction for temporal autocorrelation.

\begin{tabular}{lcc}
\hline Climatic parameters & Correlations & Probability pACF \\
\hline Annual sea level pressure & 0.69 & 0.0000 \\
Annual zonal wind & 0.54 & 0.0008 \\
Annual meridional wind & 0.57 & 0.0004 \\
Wind intensity & 0.54 & 0.0008 \\
Cloudiness & 0.02 & 0.8866 \\
Mean SST & 0.97 & 0.0000 \\
\hline
\end{tabular}




\section{Supplementary Figures}

\section{Supplementary Figure 1 | Geographical location of the marine ecological time series}

analysed in this study. 1: North Sea (CPR collection); 2: Central Baltic Sea (Gotland basin, Latvian time series); 3: Ligurian Sea, Western Mediterranean (Point B time series); 4: Northern Adriatic Sea, Eastern Mediterranean (Gulf of Trieste time series); 5: Western Atlantic - Northern area (Gulf of Maine and Georges Bank regions); 6: Western Atlantic Southern area (New England and Mid-Atlantic regions); 7: Eastern Pacific - California Current (CalCOFI data); 8: Eastern Pacific (Pacific Rim salmon data); 9: Western Pacific (Pacific Rim salmon data); 10: Western Pacific - Oyashio Current (ODATE collection); 11: Western Pacific - Transition zone (south of the Oyashio Current) (ODATE collection); 12: HOT (Hawaiian Ocean Time-series); 13: San Francisco Bay; 14: Antarctic Peninsula area (KRILLBASE database). Biological variables that are considered in each site are indicated by the letters $D$ (benthic decapods), Z (zooplankton), and F (Fish). Biological variables are indicated in Methods.

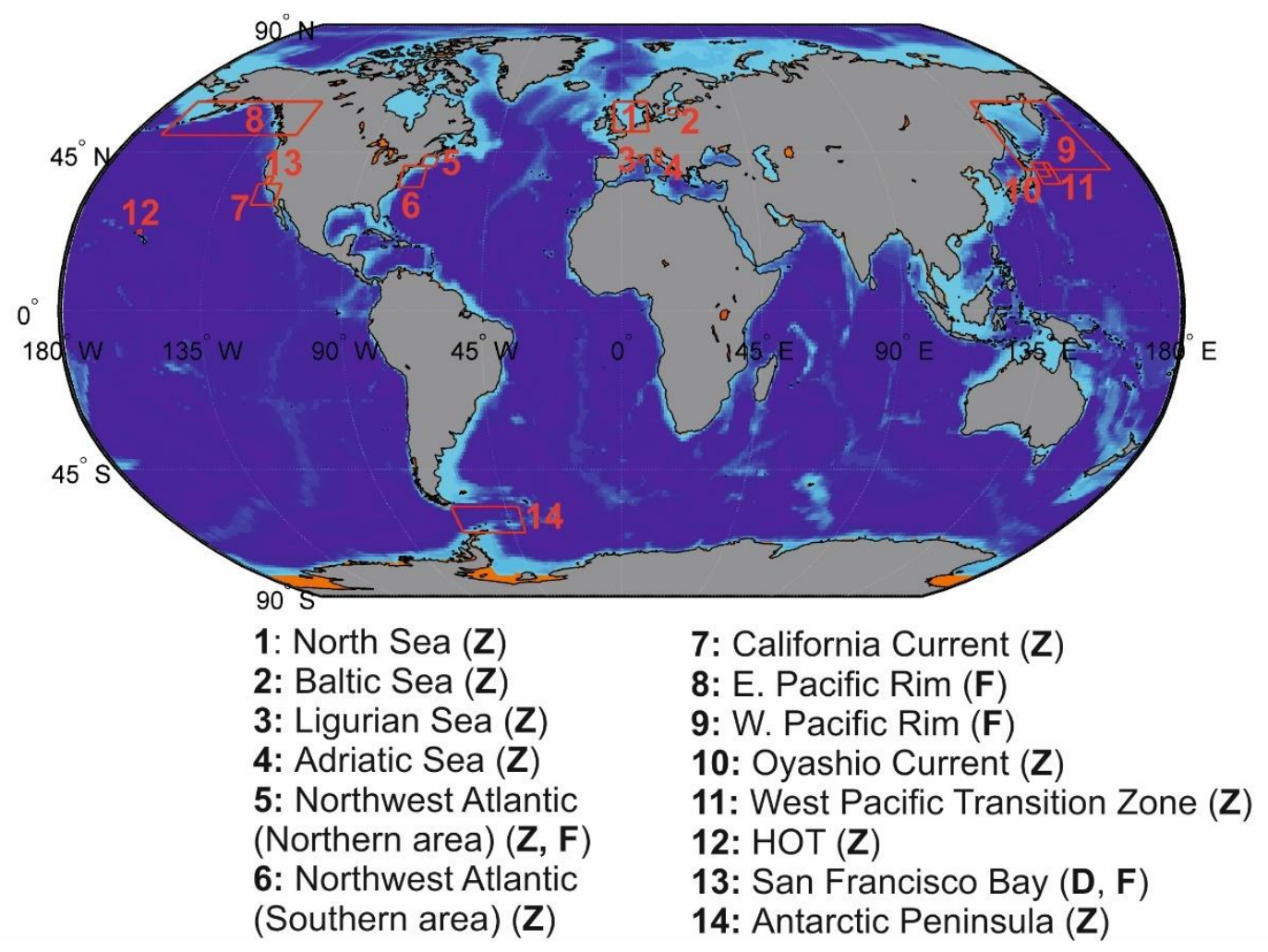


Supplementary Figure 2 | Predicted (grey) and observed (red) long-term community changes for 14 systems using a null model based on randomly generated time series with order-1 autocorrelation. Principal components were standardised between -1 and 1. Pred-PC: 10000 Principal Components (grey) based on 10000 simulated communities. Obs-PC: Principal Component based on observed communities (red). a. North Sea (Pred-PC1s and Obs-PC2), b. Baltic Sea (Pred-PC1s and Obs-PC1), c. Adriatic Sea (Pred-PC1s and Obs-PC2), d. Ligurian Sea (Pred-PC1s and Obs-PC1), e. Northwest Atlantic, southern area (Pred-PC1s and Obs-PC2), $\mathbf{f}$. Northwest Atlantic, northern area (Pred-PC1s and Obs-PC1), g. West Pacific Transition zone (Pred-PC1s and Obs-PC1), h. Oyashio (Pred-PC1s and Obs-PC1), i. CALCOFI (Pred-PC2s and Obs-PC1), j. San Francisco Bay (Pred-PC1s and Obs-PC2), k. East Pacific region (Pred-PC1s and Obs-PC1), I. West Pacific region (Pred-PC1s and Obs-PC2), m. HOT (Pred-PC2s and Obs-PC1), n. Antarctic Peninsula area (Pred-PC1s and Obs-PC2). The mean correlation is indicated. 

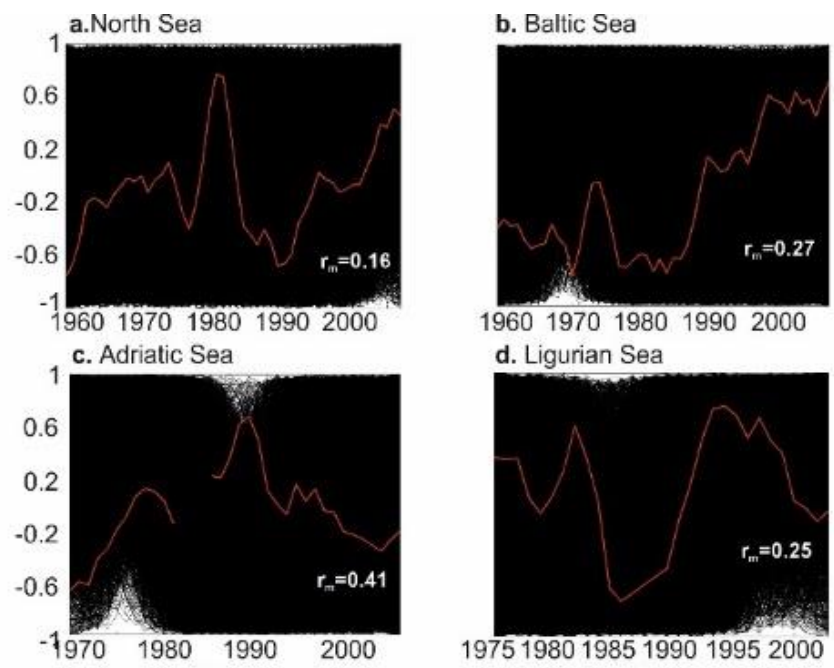

19601970198019902000

d. Ligurian Sea

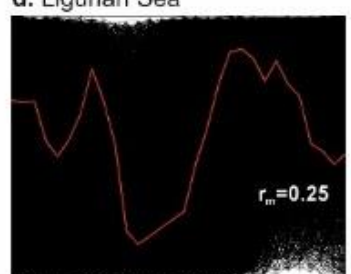

197519801985199019952000

e. Atlantic (SW)

f. Atlantic (NW)
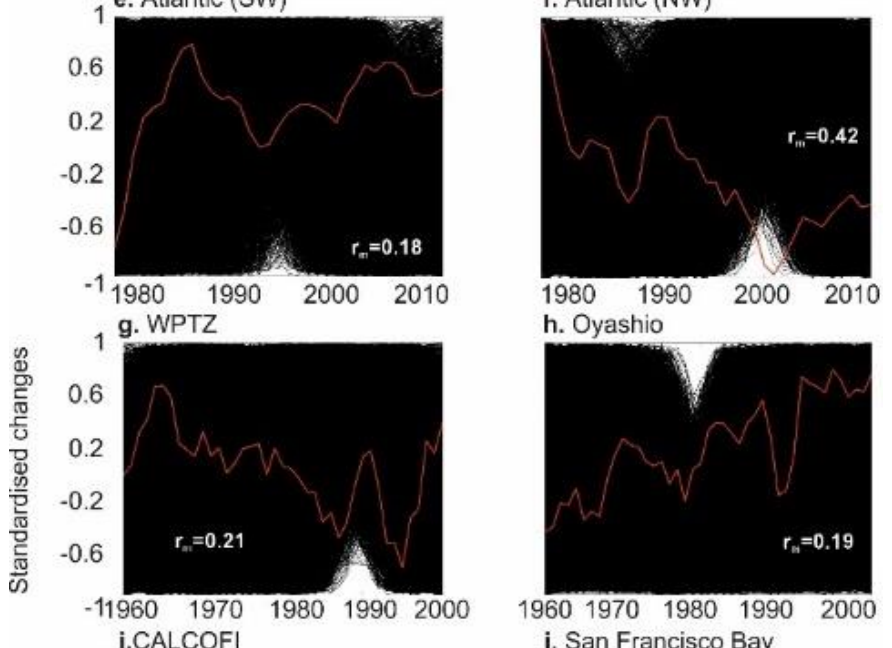

h. Oyashio
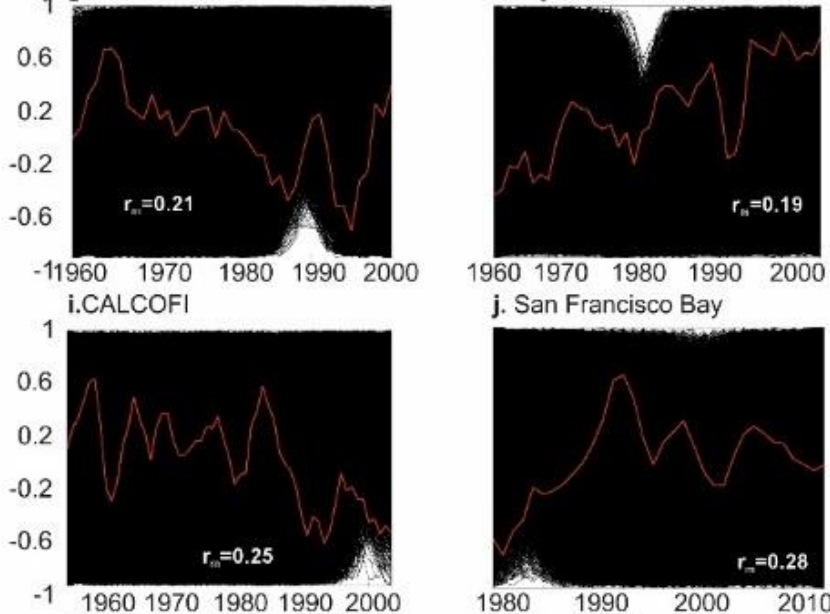

j. San Francisco Bay
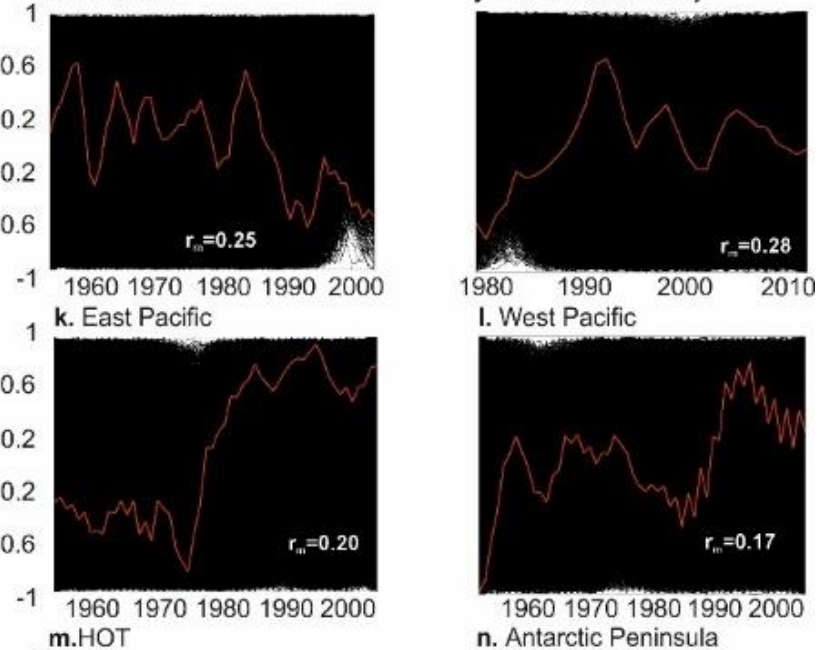

I. West Pacific

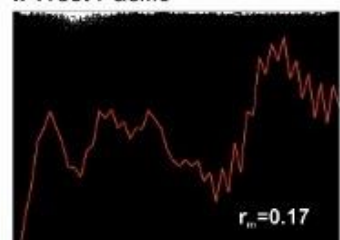

19601970198019902000 n. Antarctic Peninsula
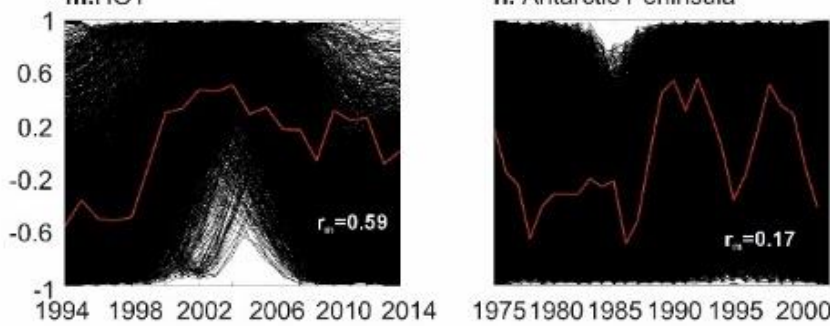

197519801985199019952000

Year

Year 
Supplementary Figure 3 | Percentage of missing data in all 28 time series (14 ObsPC1-2) from 1952 to 2014. The dashed red line shows the cut-off ( $\leq 50 \%$ of missing data) applied to select the time period 1960-2007 (vertical black arrows) to perform the standardised PCA on observed and theoretical communities.

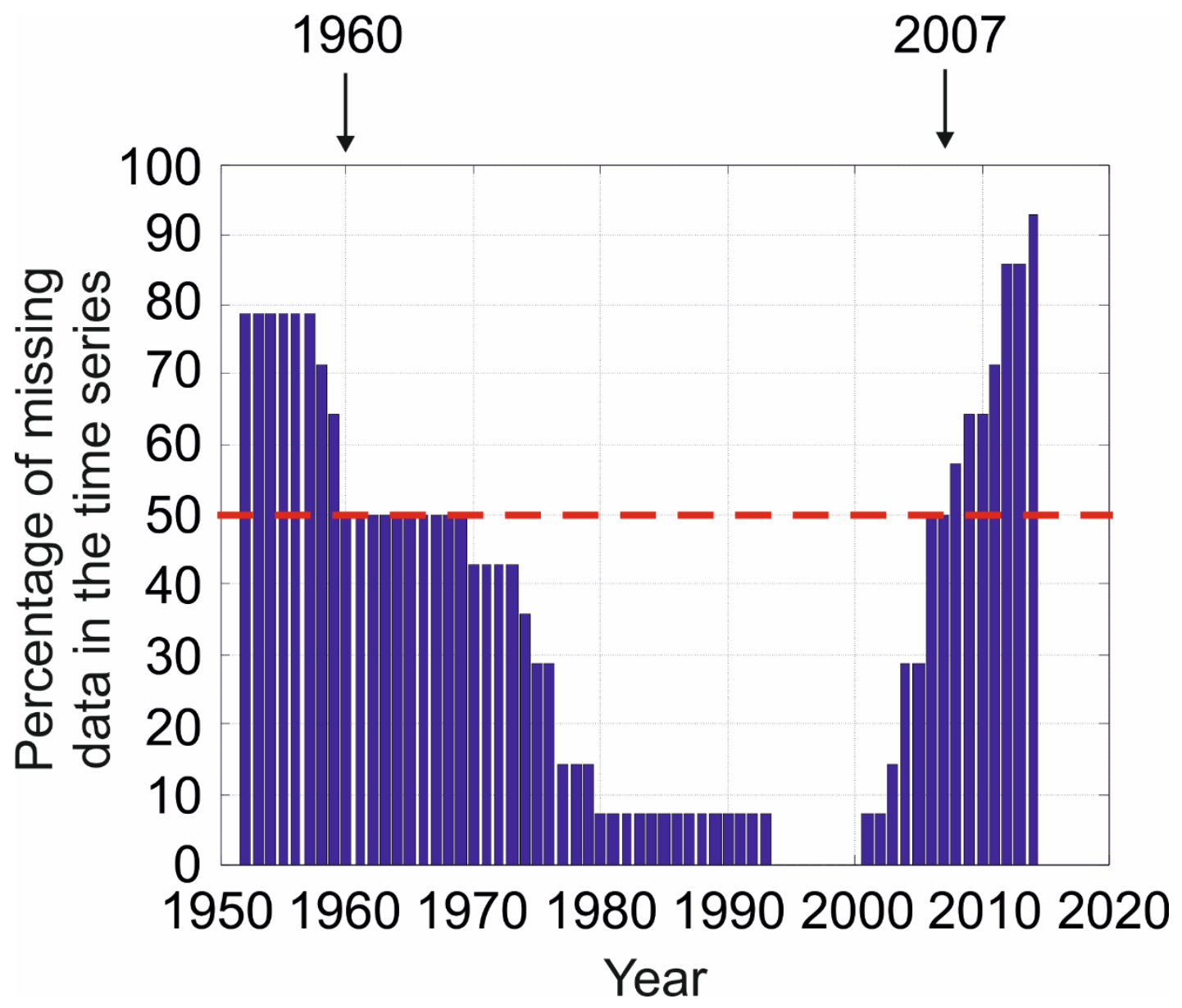


Supplementary Figure 4 | Spatial distribution of predicted Abrupt Community Shifts (ACSs) from 1960 to 2015. Maps are from 1960-1963 to 2012-2015 and originate from the application of a weighted three-order difference applied individually on each pseudo-species for every geographical cell. Values above 3 are considered to be significant and each map therefore reveals the percentage of pseudo-species that shifted in each pseudo-community. White regions are areas where no significant shifts were observed (i.e. percentage of pseudo-species' shift strictly $=0$ ). When the percentage of pseudo-species' shifts is $>0$, the percentage is indicated by a colour (blue and red for low and high percentage, respectively). 
Supplementary Figure 5 | Spatial distribution of predicted abrupt climatic shifts from 1960 to 2015. Maps are from 1960-1963 to 2012-2015 and originate from the application of a weighted three-order difference applied individually on each climatic parameter for every geographical cell. Values above 3 are considered to be significant and each map therefore reveals the percentage of climatic parameters (total $=6$ ) that shifted in geographical cell. White regions are areas where no significant shifts were observed (i.e. percentage of shift strictly $=0$ ). Climatic parameters were annual sea level pressure, meridional and zonal wind, wind intensity, cloudiness and annual sea surface temperature. 
Supplementary Figure 6 | Spatial patterns in abrupt shifts of six climatic parameters centred on the period 2010-2014. a. Annual Sea Level Pressure (SLP). b. Annual wind intensity. c. Annual zonal (U) wind. d. Annual meridional (V) wind. e. Cloudiness. f. Annual Sea Surface Temperature (SST). Values above 3 show significant acceleration phases. The centred period 2010-2014 integrates years ranging from 2008 to 2015 to calculate acceleration phases.
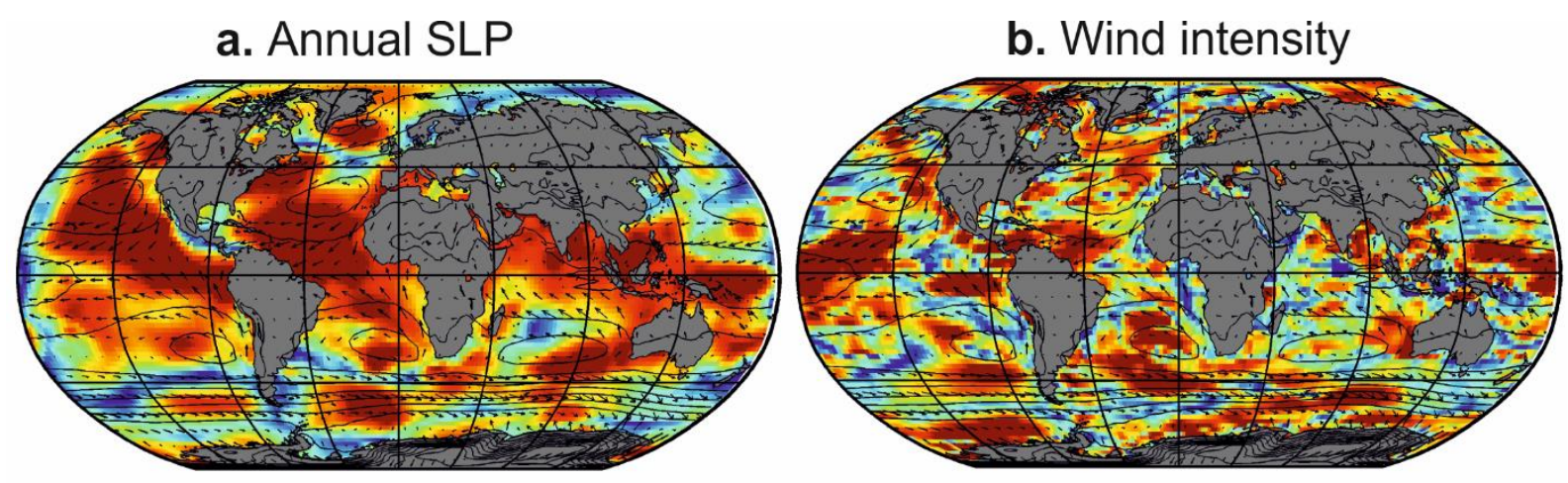

c. Zonal (U) wind

\section{d. Meridional (V) wind}
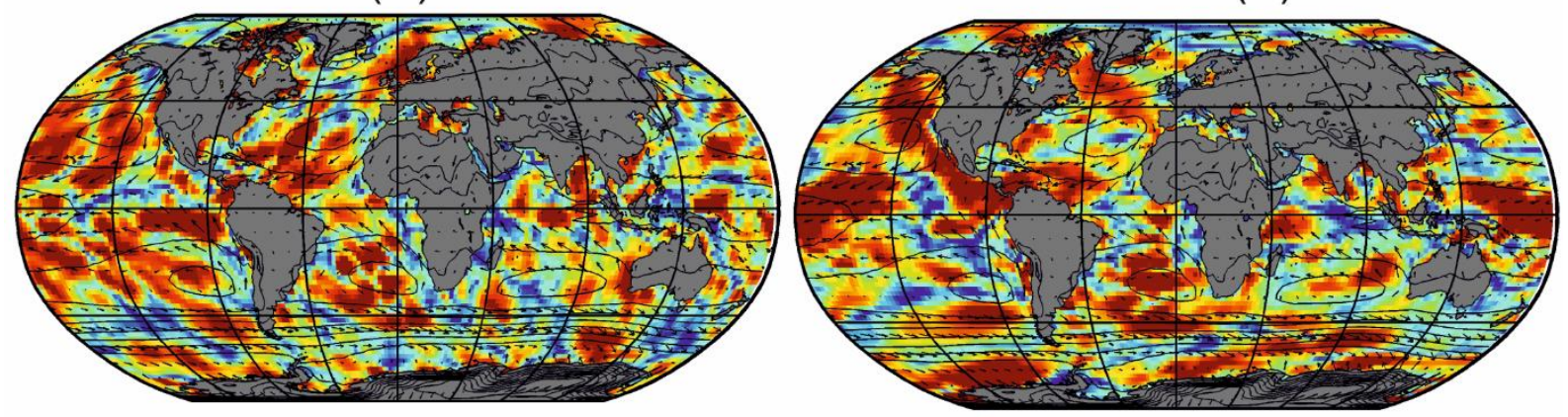

e. Cloudiness

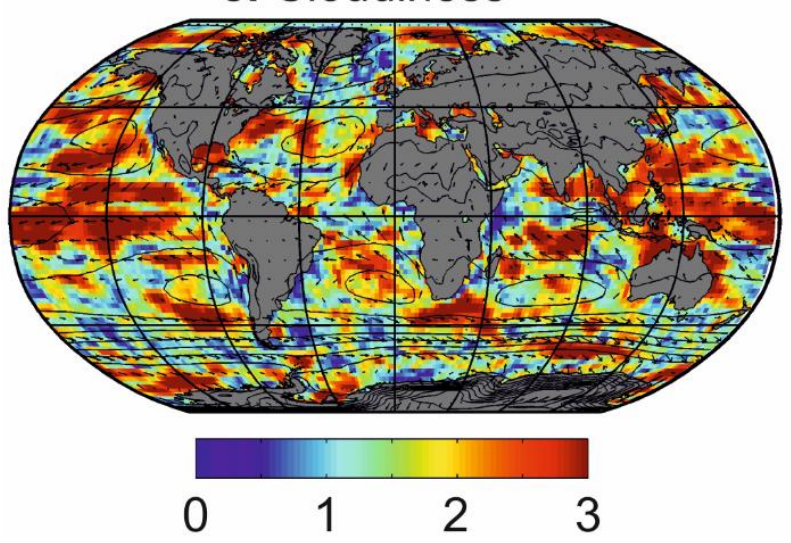

Index of abruptness

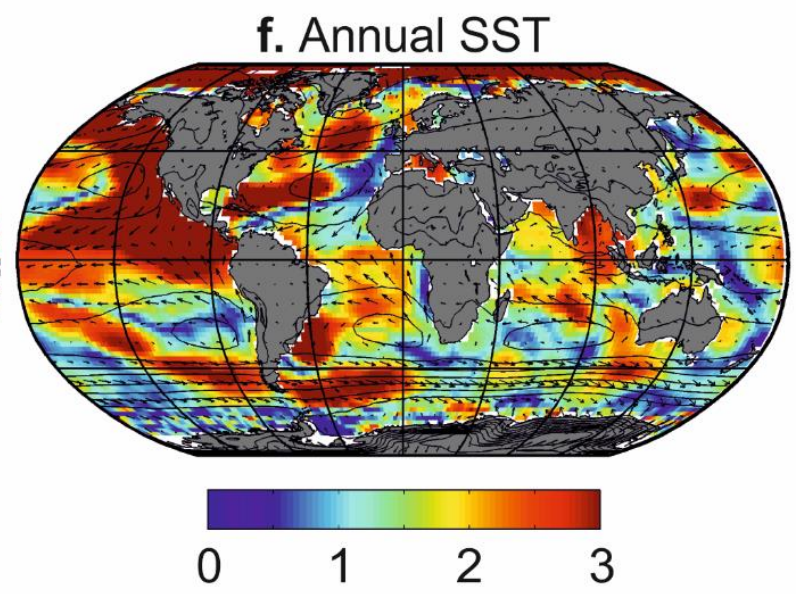

Index of abruptness 
Supplementary Figure 7 | Summary of the procedure used to build pseudo-communities in the ocean from the METAL theory, test the framework against observed data and forecast ACSs at a global scale. Step 1. A pool of pseudo-species is created, each pseudo-species being characterized by a unique thermal niche. A total of 19609 pseudo-species was randomly chosen. Step 2. Sea Surface Temperature (SST) were organized for a grid of $2^{\circ}$ latitude $\times 2^{\circ}$ longitude for each month and year of the period 1960-2015. Step 3. The interaction between the species' thermal niche and year-to-year monthly SST determine the species richness locally. Changes in the local abundance of a pseudo-species were assessed by linear interpolation of SSTs from pseudo-species' thermal niche. Step 4. Long-term changes were estimated by averaging the expected monthly abundance of each pseudo-species, the type of averaging depending upon observed biological data (monthly, 2-, 3-, and 4-month periods or annual averaging). Testing of the framework with observed long-term time series. Step 5. Long-term changes in the state of each local pseudo-community were assessed from standardised principal components analyses. Step 6. Use of the algorithm of abrupt shift detection on principal components. Prediction of abrupt community shifts. Step 7. Detection of abrupt shifts for each pseudo-species and in each geographical cell. Step 8. Maps of ACSs, defined as the percentage of pseudo-species showing a shift, for each geographical cell and time period. 
1. Creation of a global pool of pseudo-species (19609)

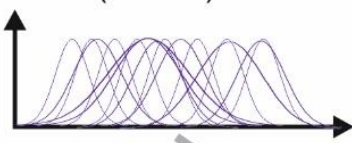

3. Interaction between the thermal niche

of a pseudo-species and changes in SST determines

the presence of a pseudo-species in a local pseudo-community,

pseudo-species richness and monthly and

year-to-year fluctuations in the pseudo- species

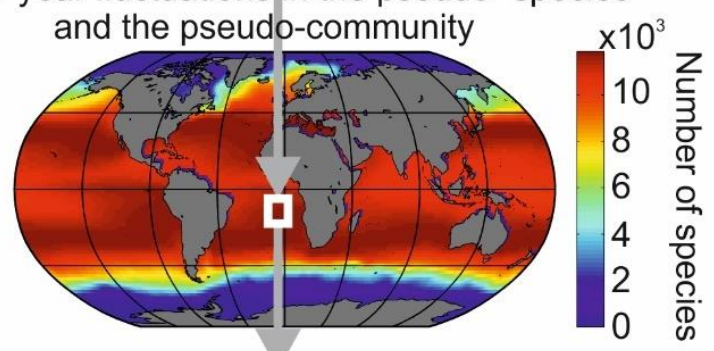

4. Predicted long-term changes

of pseudo-species for each month and year (1960-2015)

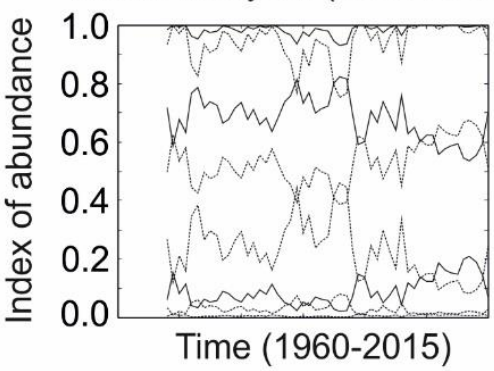

Testing of the framework with observed

long-term time series

5. PCA to summarise the त state of the pseudo-community

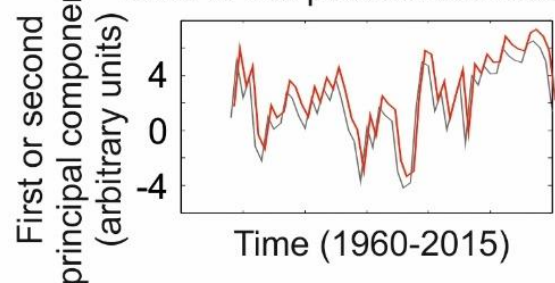

6. Detection of Abrupt shifts in both detected and observed time series

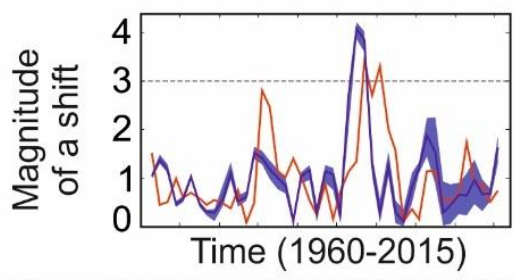

\section{Prediction of abrupt community shifts}

7. Detection of Abrupt shifts for each pseudo-species and geographical cells :

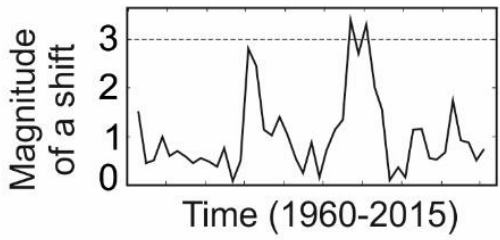

8. Maps of ACSs for each time period ( $\%$ of species with a substantial shift)

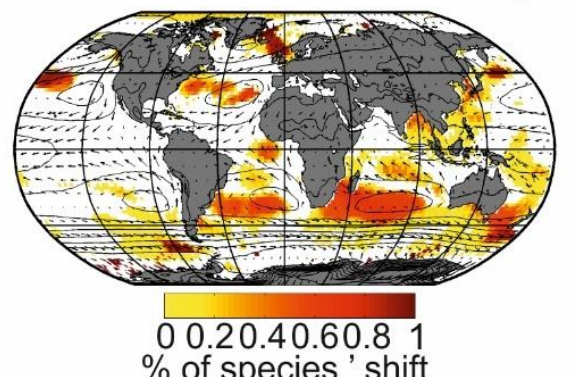


Supplementary Figure 8 | Application of the abrupt shift detection algorithm to a fictive time series with a white noise (maximum amplitude of 1). a. Simulated time series (in black) and application of an order-1 symmetrical moving average (red dashed line). b-c. Index of abruptness based on order-1-5 weighted differences with (b) no or (c) an application of an order-1 symmetrical moving average prior to the use of the abrupt shift detection algorithm. The red dashed line represents the threshold of 3 of significance.

a. Simulated time series

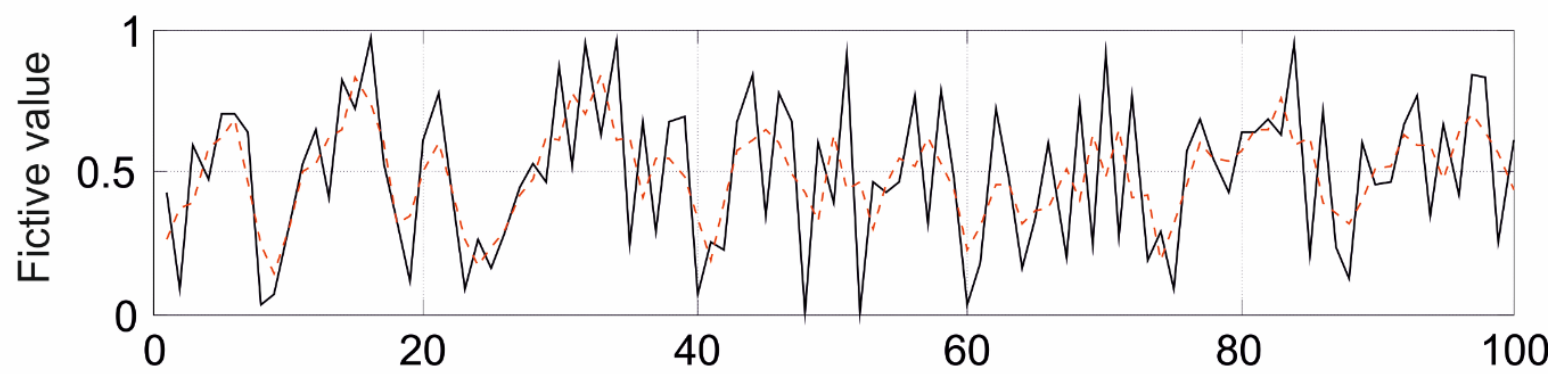

b. Index of abruptness (order 1-5 difference, no moving average)

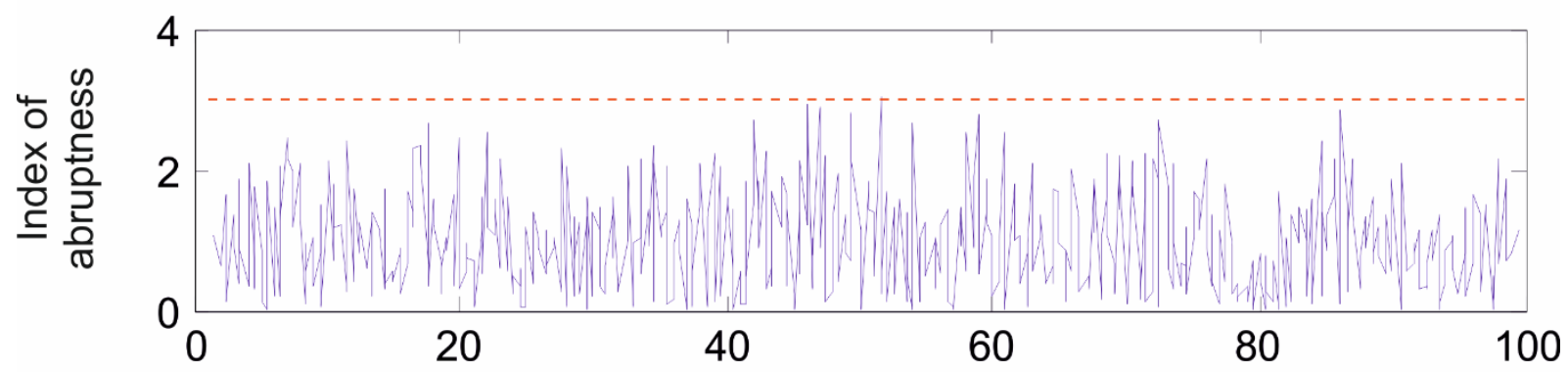

c. Index of abruptness (order 1-5 difference, moving average)

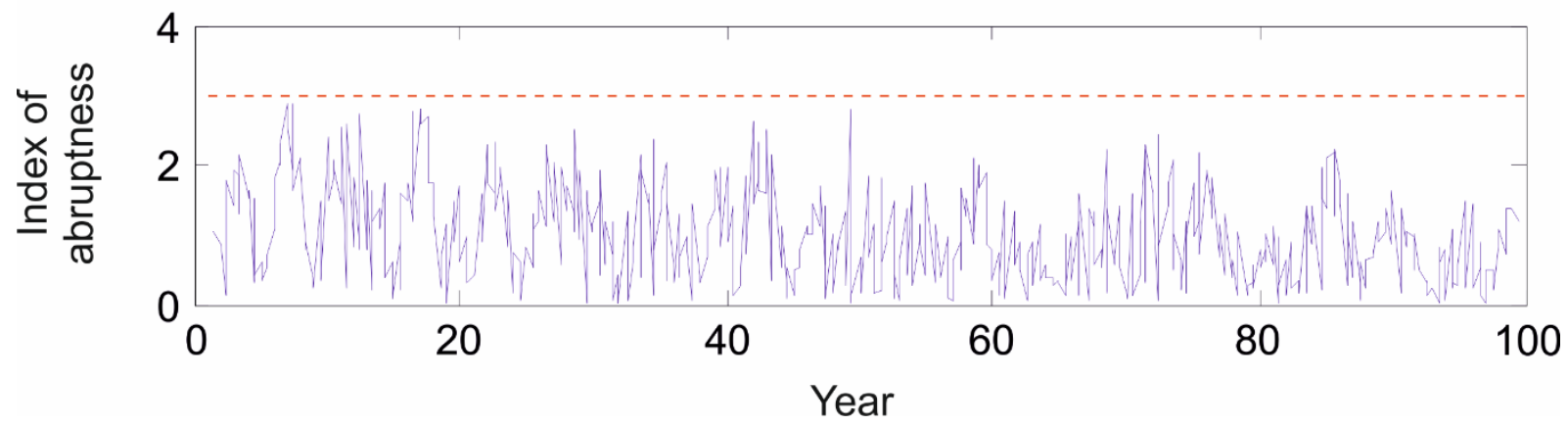


Supplementary Figure 9 | Application of the abrupt shift detection algorithm to a fictive time series with a linear trend (amplitude $\mathbf{0 . 5}$ ) and a white noise (maximum amplitude of 1). a. Simulated time series (in black) and application of an order-1 symmetrical moving average (red dashed line). The blue dashed line shows the linear trend. b-c. Index of abruptness based on order-1-5 weighted differences with (b) no or (c) an application of an order-1 symmetrical moving average prior to the use of the abrupt shift detection algorithm. The red dashed line represents the threshold of 3 of significance.

a. Simulated time series

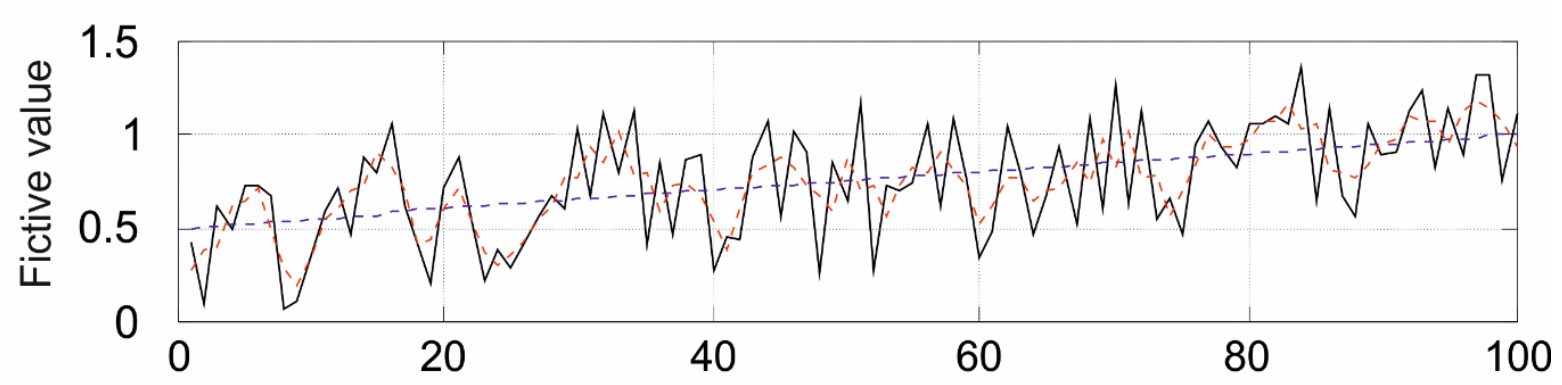

b. Index of abruptness (order 1-5 difference, no moving average)

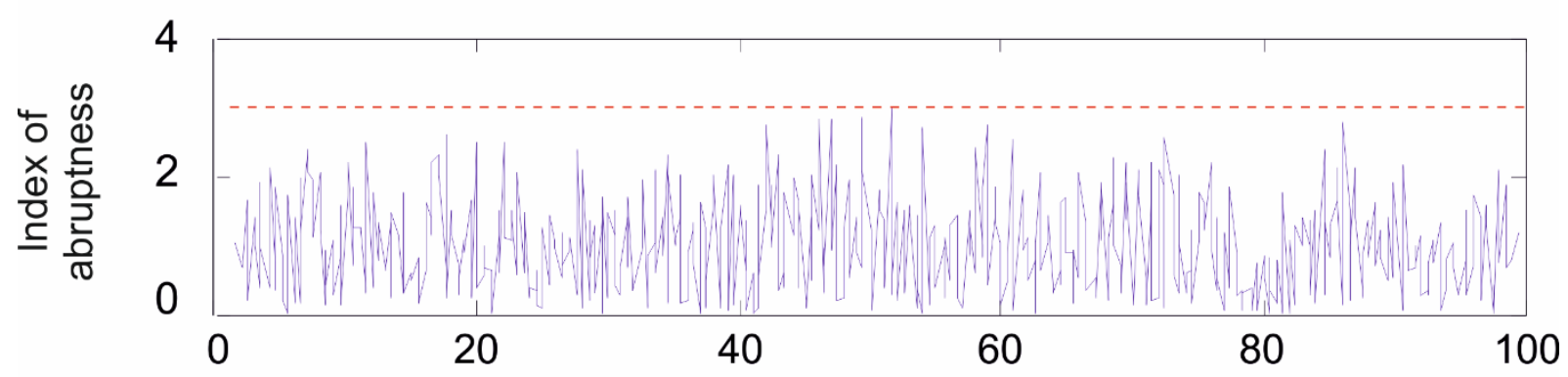

c. Index of abruptness (order 1-5 difference, moving average)

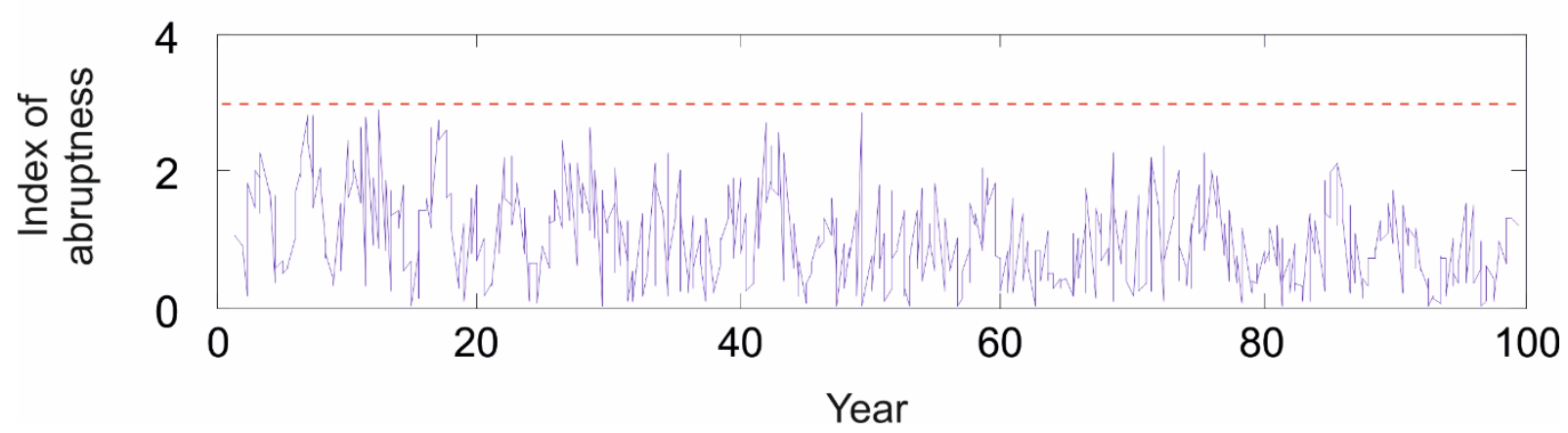


Supplementary Figure 10 | Application of the abrupt shift detection algorithm to a fictive time series with a linear trend (amplitude $=0.5$ ), a cycle (amplitude $=1$ ) and a white noise (maximum amplitude of 1). a. Simulated time series (in black) and application of an order-1 symmetrical moving average (red dashed line). The blue dashed curve highlights the cycle. $\mathbf{b}$ c. Index of abruptness based on order-1-5 weighted differences with (b) no or (c) an application of an order-1 symmetrical moving average prior to the use of the abrupt shift detection algorithm. The red dashed line represents the threshold of 3 of significance.

a. Simulated time series

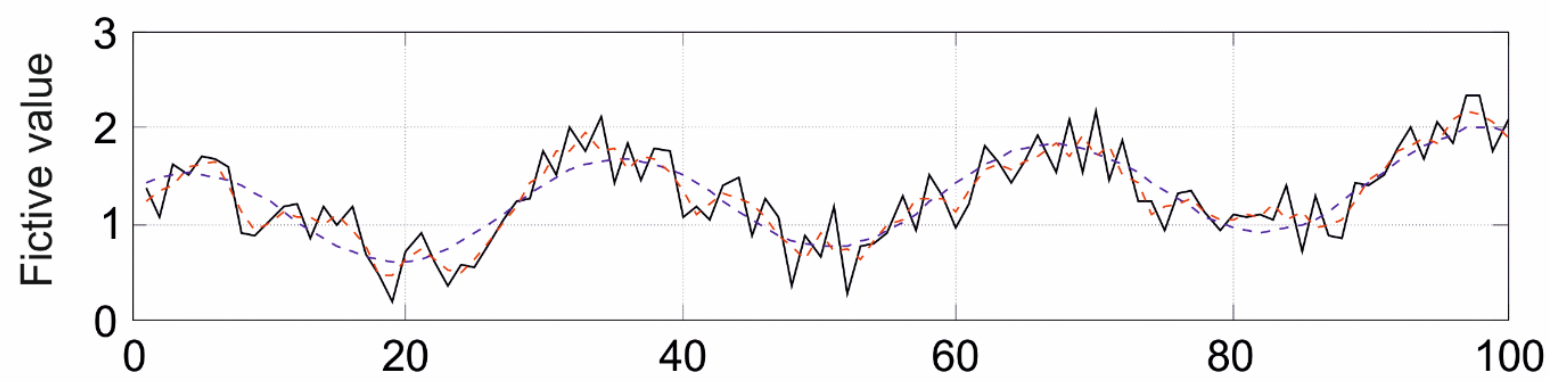

b. Index of abruptness (order 1-5 difference, no moving average)

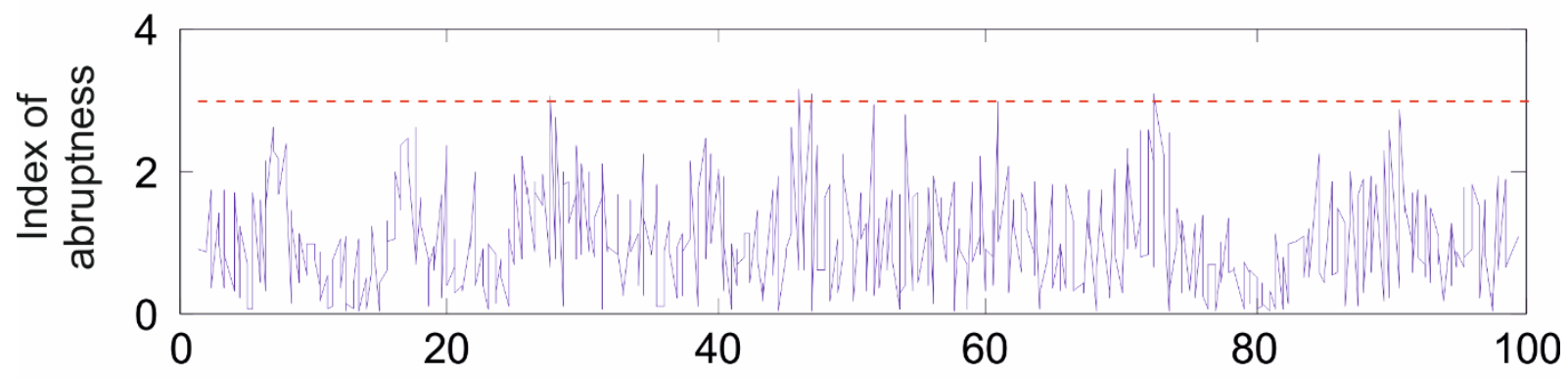

c. Index of abruptness (order 1-5 difference, moving average)

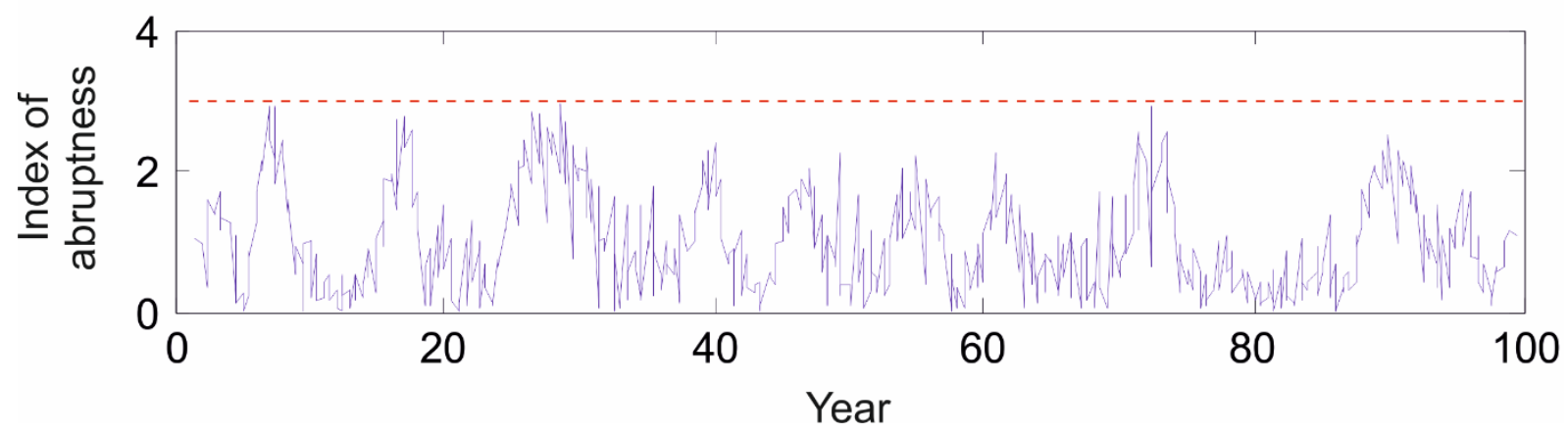


Supplementary Figure 11 | Application of the abrupt shift detection algorithm to a fictive time series with a linear trend (amplitude $=0.5$ ), a cycle (amplitude $=1$ ), an abrupt shift (amplitude $=1$ ) and a white noise (maximum amplitude of 1 ). a. Simulated time series (in black) and application of an order-1 symmetrical moving average (red dashed line). The blue dashed lines highlight the abrupt shift. b-c. Index of abruptness based on order-1-5 weighted differences with (b) no or (c) an application of an order-1 symmetrical moving average prior to the use of the abrupt shift detection algorithm. The red dashed line represents the threshold of 3 of significance. The black arrow shows the position of the abrupt shift.

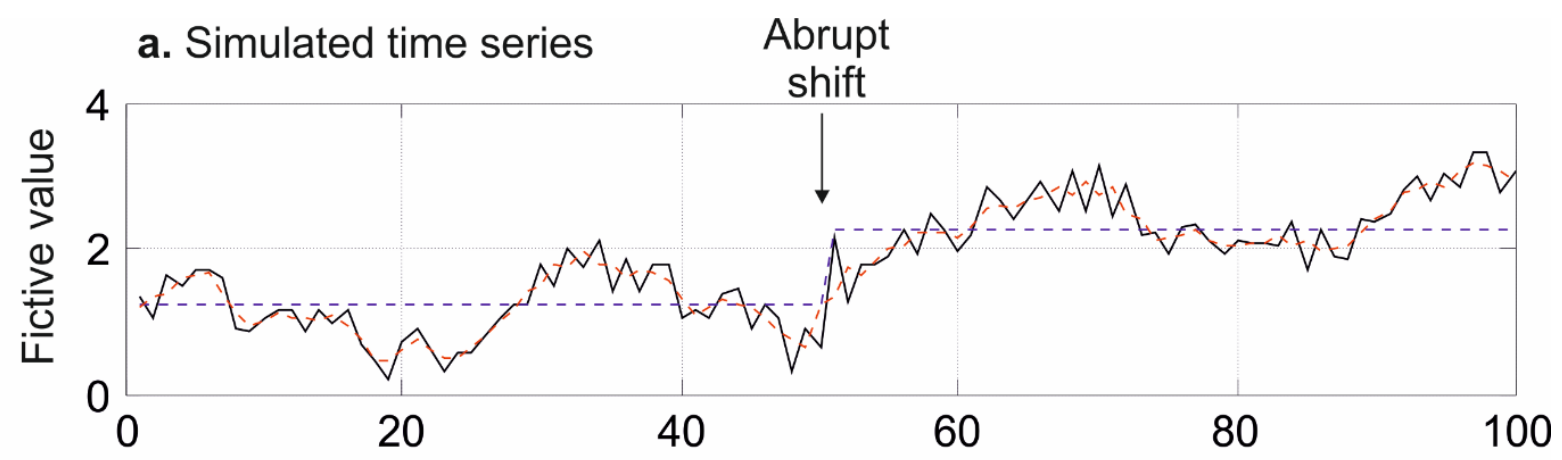

b. Index of abruptness (order 1-5 difference, no moving average)

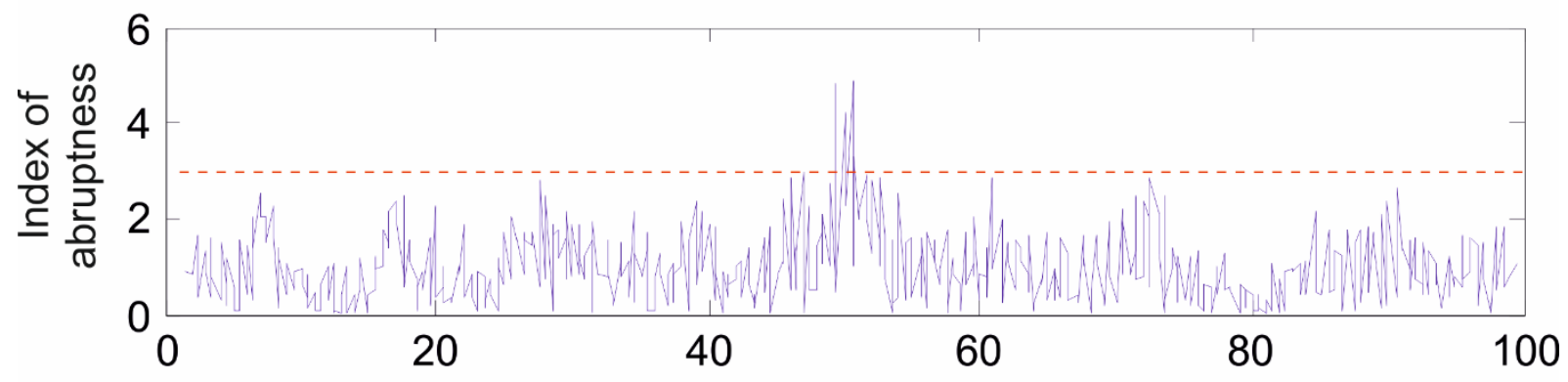

c. Index of abruptness (order 1-5 difference, moving average)

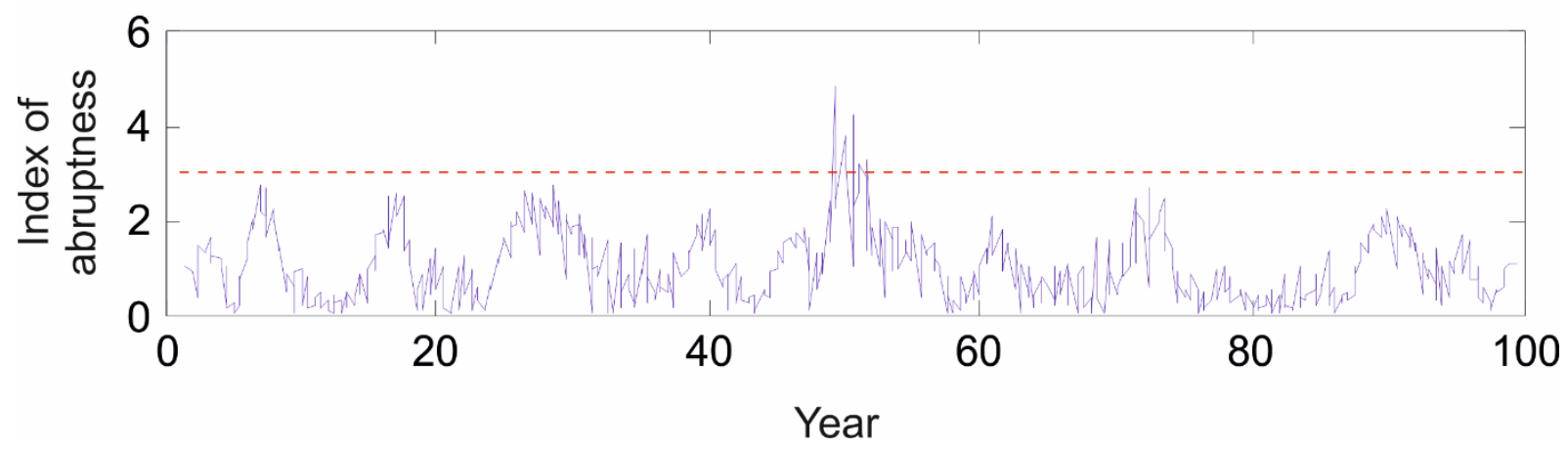


Supplementary Figure 12 | Application of the abrupt shift detection algorithm to a fictive time series with a linear trend (amplitude $=0.5$ ), a cycle (amplitude $=1$ ), an abrupt shift (amplitude $=1$ ), an episodic event (amplitude of 1 ) and a white noise (maximum amplitude of 1). a. Simulated time series (in black) and application of an order-1 symmetrical moving average (red dashed line). The blue dashed lines highlight the abrupt shift and the episodic event. b-c. Index of abruptness based on order-1-5 weighted differences with (b) no or (c) an application of an order-1 symmetrical moving average prior to the use of the abrupt shift detection algorithm. The red dashed line represents the threshold of 3 of significance. The black arrows highlight the position of the abrupt shift and the episodic event.
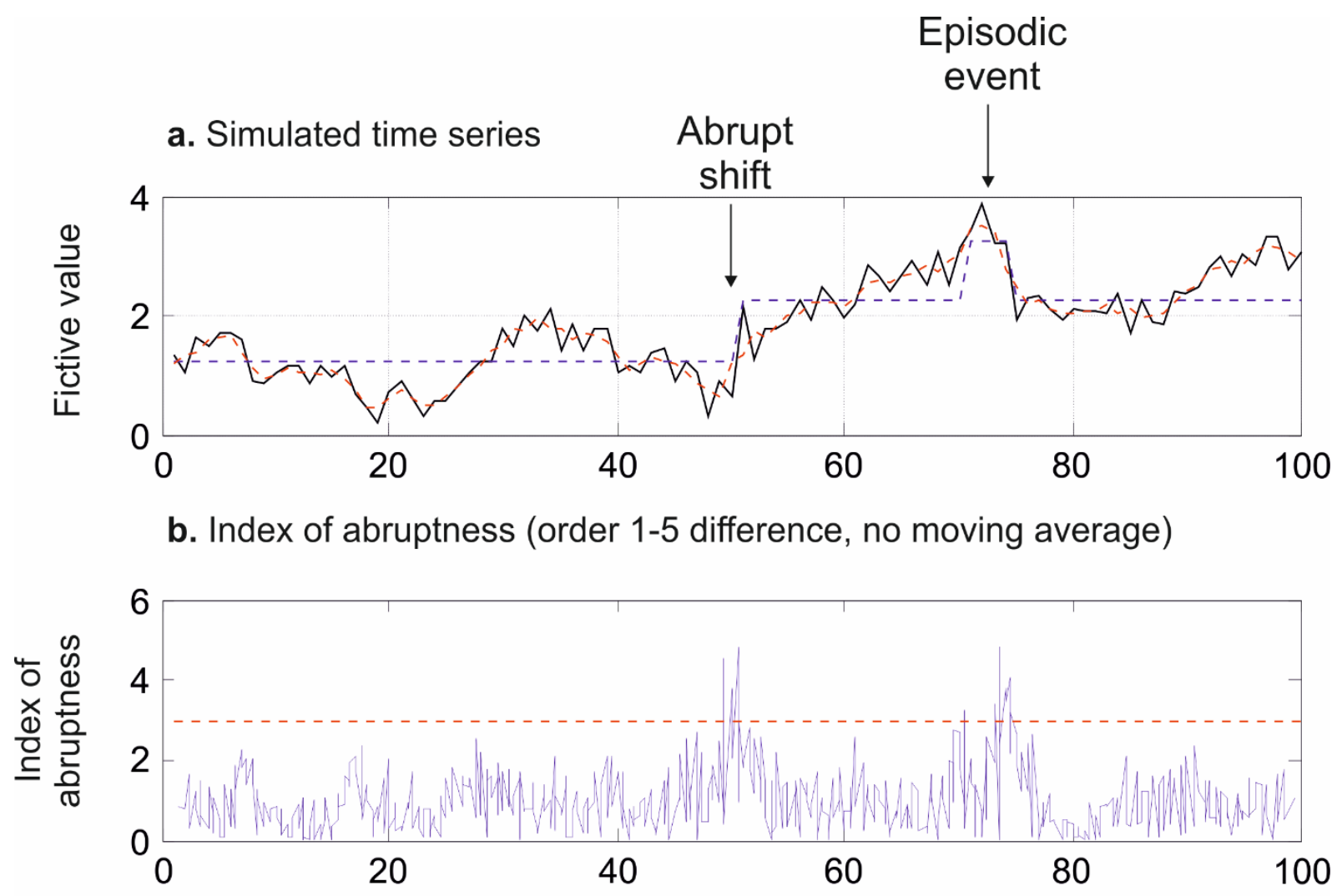

c. Index of abruptness (order 1-5 difference, moving average)

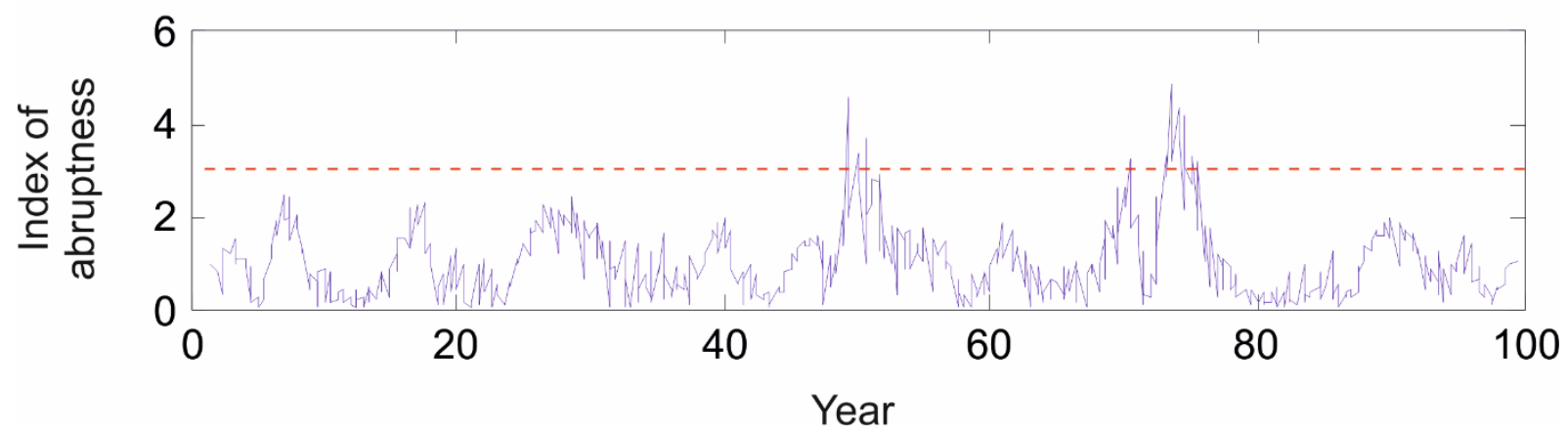


Supplementary Figure 13 | Autocorrelograms of time series used in Supplementary Figure $\mathbf{8}$ (top) and $\mathbf{9}$ (bottom). On the left, autocorrelograms are calculated on original time series (no moving average) and on the right autocorrelograms are calculated after the application of a first-order symmetrical moving average to reduce the influence of white noise. The $95 \%$ confidence interval is included by a dashed curve (here we consider the removal of the degree of freedom for each lag). It should be noted that the linear trend is also included in Supplementary Figure 10-12.

No moving average

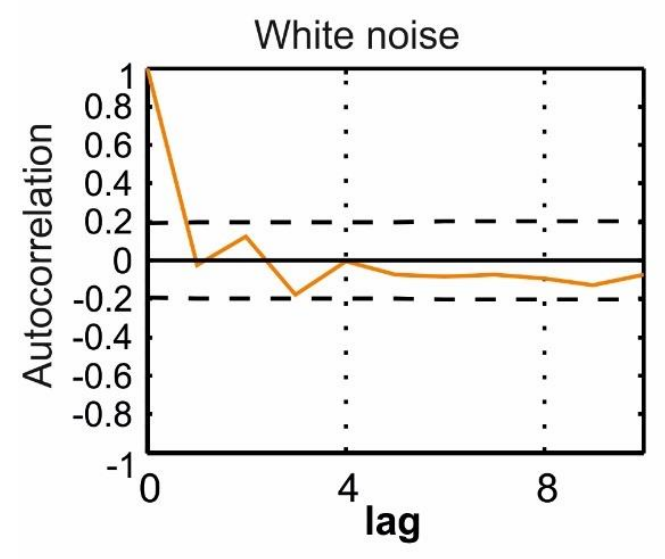

White noise

+ linear trend

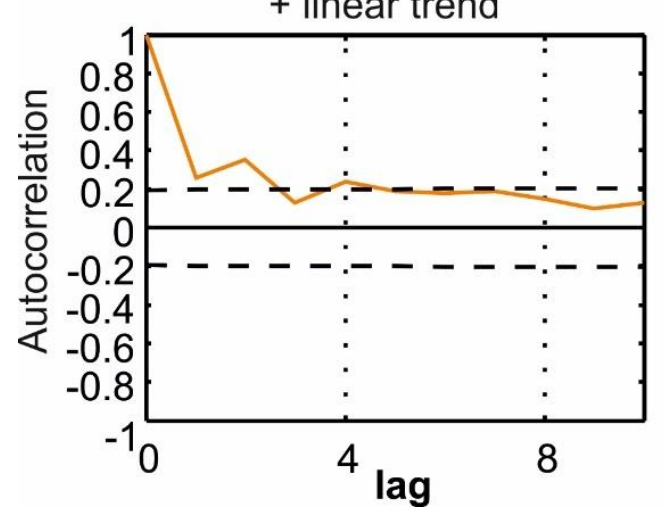

First-order symmetrical moving average

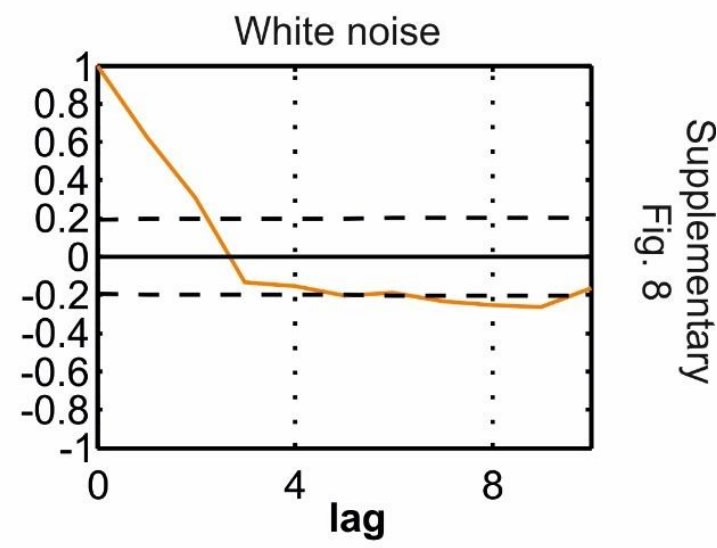

White noise

+ linear trend

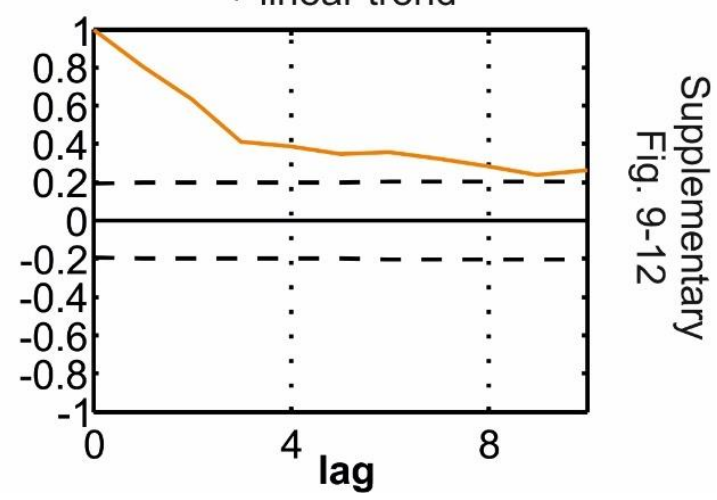


Supplementary Figure 14 | Schematic diagram summarizing the numerical procedures used to compare observed long-term biological changes with those expected from the null and METAL models (see Figures 2 and 3). We performed standardized Principal Components Analyses (PCAs) to summarize the long-term observed biological changes in each system (14 systems), using the first two Principal Components (PCs). The same procedure was applied for the two null and the METAL models; the building of the pseudo-communities and the calculation of the corresponding standardised PCA was repeated 10000 times for each system. All data were subsequently aggregated into a matrix (Tables D-G) to examine (i) the correlations between observed biological changes and those expected from the null and METAL models using one PC (10000 simulations), (ii) the relationships between observed biological changes and the first two expected PCs (10000 simulations) and (iii) the examination of the first three PCs originating from a global PCA performed on aggregated data (10000 simulations, Tables D-F, see text). 
Observed biological systems

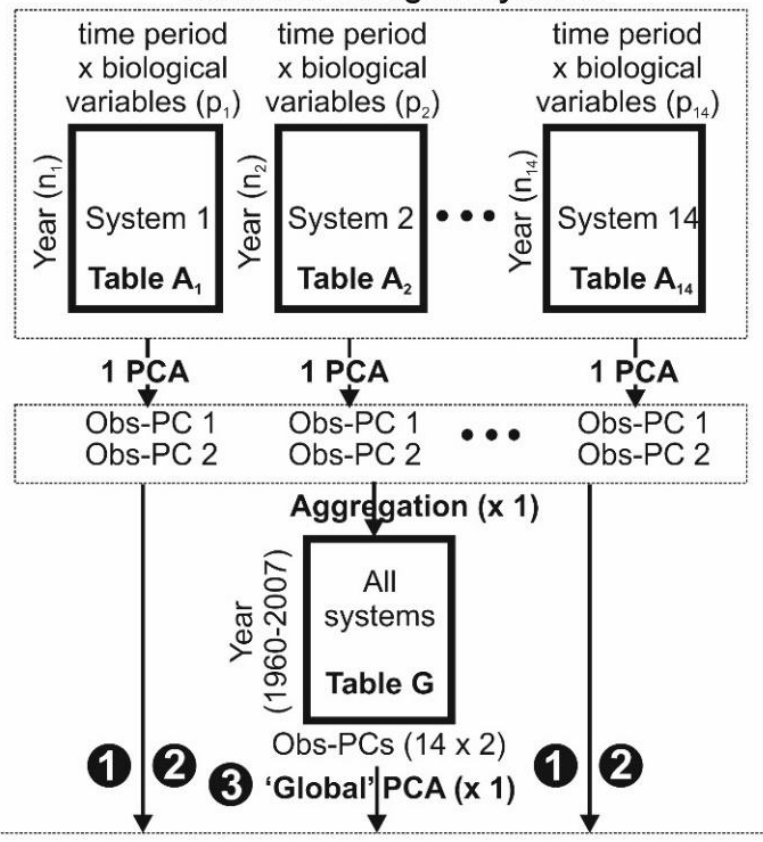

(1) Examples of best correlations between Pred-PC1-2s (METAL and null models) and Obs-PC1-2s (Figure 1a-n) 2Regressions on first 2 Pred-PC1-2s (METAL and null models) and selected Obs-PC (Figure 1o-p)

3Relationships between 'global' PC1-3s (METAL and type-2 null model) and detection of ACSs (Figure 2)

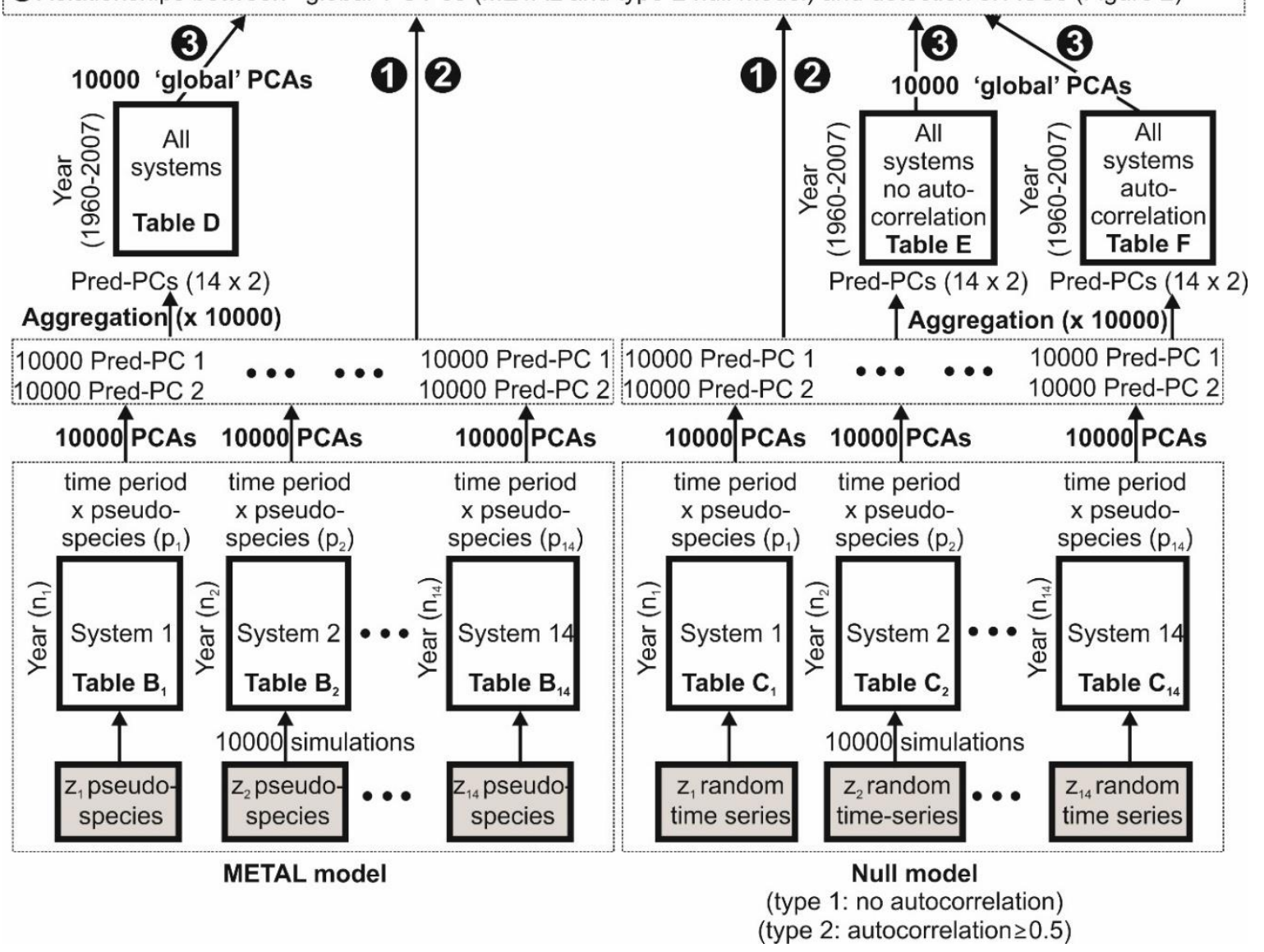


Supplementary Figure 15 | Histogram showing the number of geographical cells covered by our framework according to each category of depth from 5000 to $0 \mathrm{~m}$ (every $100 \mathrm{~m}$ ).

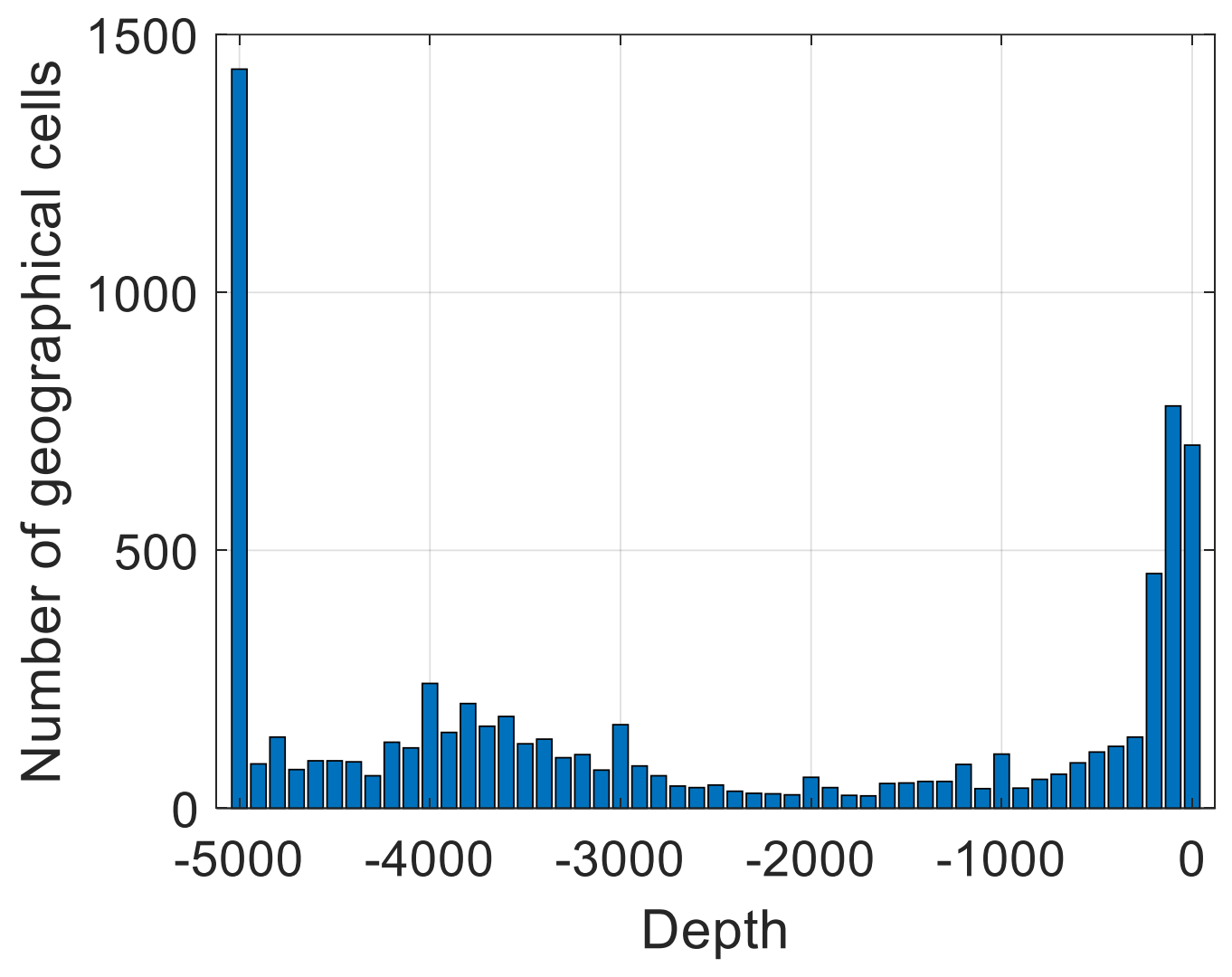


Supplementary Figure 16 | Correlation between observed and predicted principal components (PCS of Figures 2a-n) as a function of the surface area $\left(\mathrm{km}^{2}\right)$ and the mean bathymetry $(\mathrm{m})$ of each region. The size and colour of the circles are proportional to the value of the correlation. Because of the relatively low spatial resolution of our framework, some time series (e.g. Ligurian Sea, Point B) were considered as representative of a larger area (last column in Supplementary Table 3).

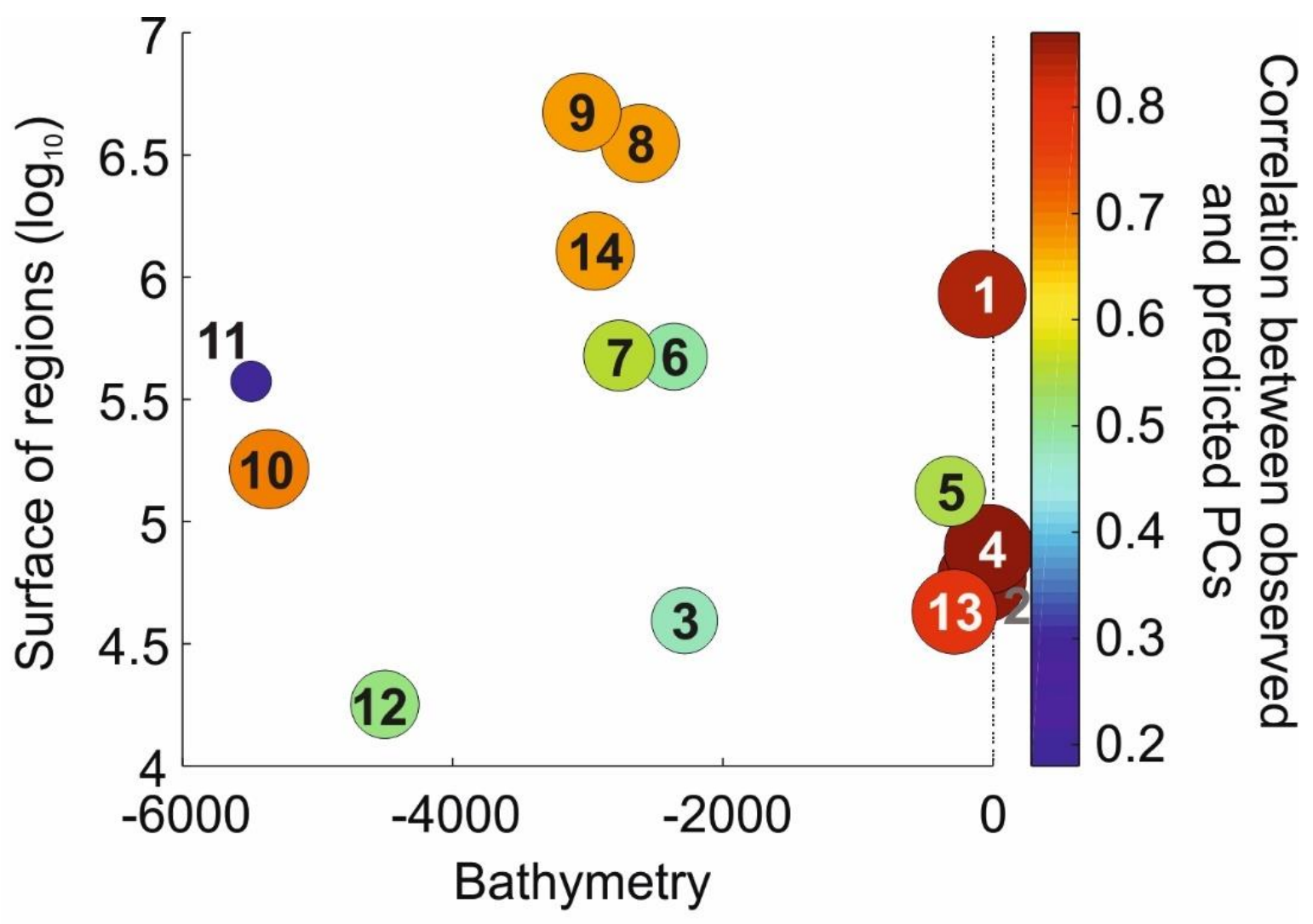

1: North Sea

2: Baltic Sea

3: Ligurian Sea

4: Adriatic Sea

5: NW Atlantic (north)

6: NW Atlantic (south)

7: California Current
8: E Pacific Rim

9: W Pacific Rim

10: Oyashio Current

11: Transition Zone

12: HOT

13: San Francisco

14: Antarctic Peninsula 
Supplementary Figure 17 | Predicted (black) and observed (red) long-term community changes for 5 offshore areas sampled by the Continuous Plankton Recorder (CPR) survey. First Principal Components (PCs) were standardised between -1 and 1. The 10,000 Principal Components (black) were based on 10,000 simulated communities. Left panels show predicted (black) and observed (red) community changes. $r_{m}$ : mean correlation between predicted and observed changes. Inner panels show the spatial distribution of CPR samples (red dot) in the North Atlantic Ocean. Middle and right panels show the cumulative frequency histograms of the bathymetry (middle) and distance to land (right) of each selected CPR sample. (a) Bay of Biscay ( 75 species or taxa, $8-4^{\circ} \mathrm{W}$ and $44-48^{\circ} \mathrm{N}$, observed PC1: $15.16 \%$ of the total variance), (b) Western oceanic part of the United Kingdom (60 species or taxa, $19-14^{\circ} \mathrm{W}$ and $48-54^{\circ} \mathrm{N}$, observed PC1: $12.19 \%$ of the total variance), (c) Faroe-Iceland Rise (51 species or taxa, $15-5^{\circ} \mathrm{W}$ and $60-65^{\circ} \mathrm{N}$, observed PC1: $14.28 \%$ of the total variance), (d) Denmark Strait (49 species or taxa, $40-20^{\circ} \mathrm{W}$ and $60-65^{\circ} \mathrm{N}$, observed PC1: $17.79 \%$ of the total variance), (e) Labrador Basin (52 species or taxa, $60-40^{\circ} \mathrm{W}$ and $50-55^{\circ} \mathrm{N}$, observed PC1: $17.43 \%$ of the total variance). All changes were significant at $p<0.001$ and $p \leq 0.1$ when temporal autocorrelation was taken into account. $\mathrm{n}$ is the number of CPR samples. All first PCs were significant after the use of a broken-stick distribution (Methods). 

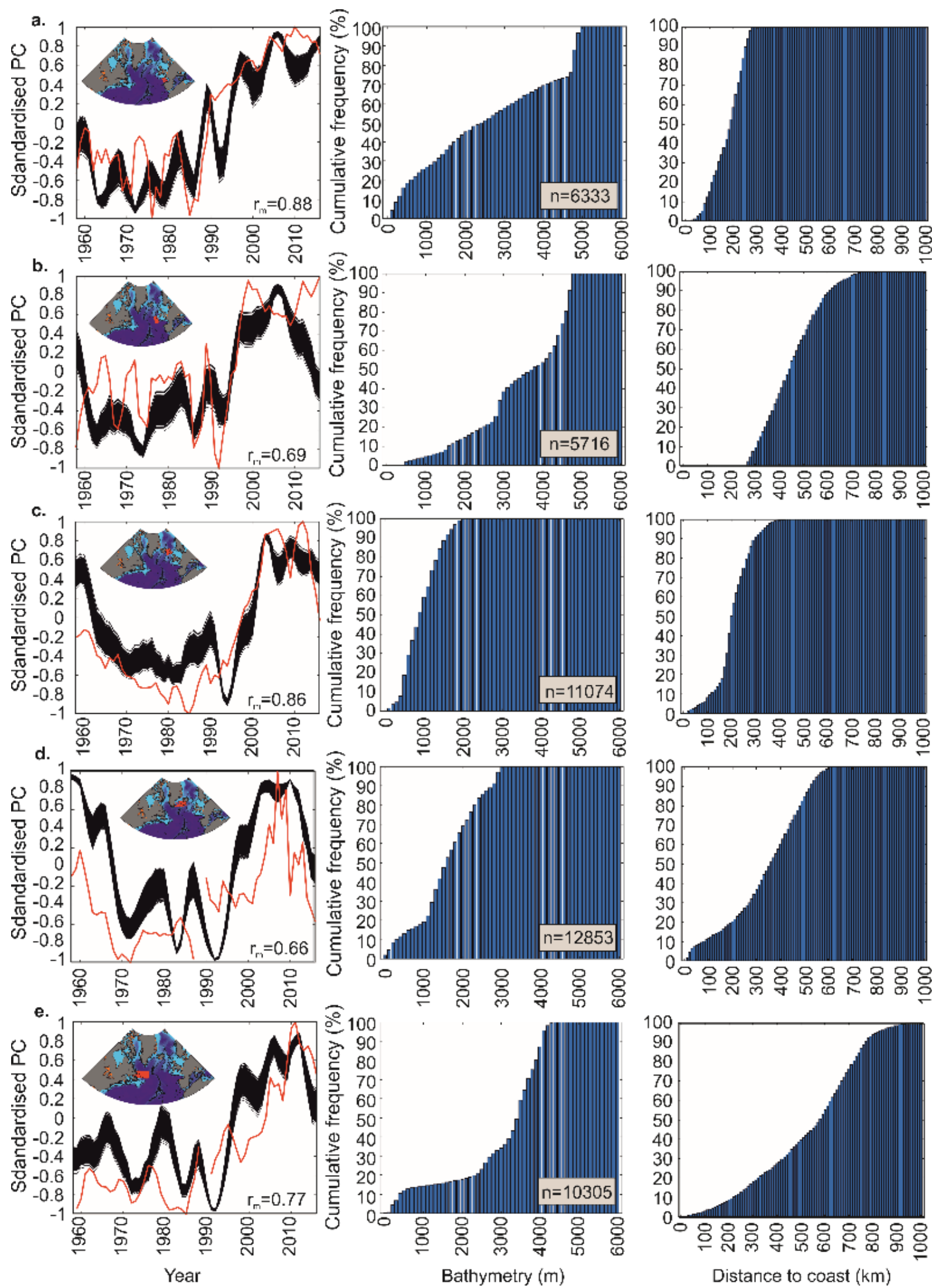
Supplementary Figure 18 | Effect of the reduction of the number of years on the correlation between observed (red) and predicted (blue) principal components (see Figure $2 \mathrm{~g}$ ). The correlation was calculated by removing progressively years at the beginning of the time series from 1 to 31 years (upper panel). Left lower panel: correlations based on year 8 (1967) to end (2000). Right lower panel: correlations based on years 24 (1983) to end (2000). The probability of the correlation was adjusted to account for temporal autocorrelation.

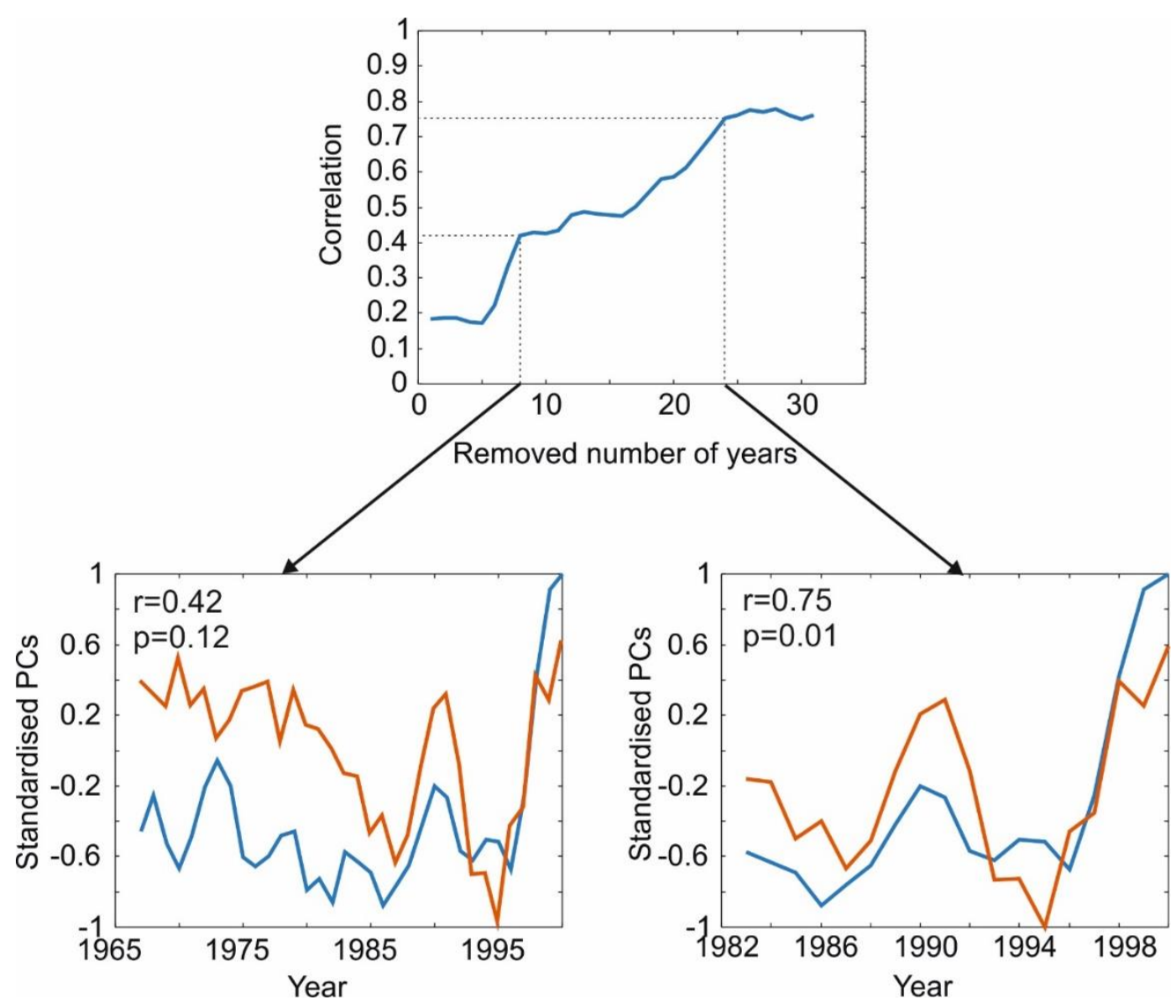




\section{Literature cited}

1 Planque, B. \& Arneberg, P. Principal component analyses for integrated ecosystem assessments may primarily reflect methodological artefacts. ICES Journal of Marine Science 75, 1021-1028 (2018).

2 Beaugrand, G., Harlay, X. \& Edwards, M. Detecting plankton shifts in the North Sea: a new abrupt ecosystem shift between 1996 and 2003. Marine Ecology Progress Series 502, 85-104, doi:10.3354/meps10693 (2014).

3 Luczak, C., Beaugrand, G., Jaffré, M. \& Lenoir, S. Climate change impact on Balearic Shearwater through a trophic cascade. Biology Letters 7, 702-705 (2011).

4 Reid, P. C. et al. Global impacts of the 1980s regime shift. Global Change Biology 22, 682-703, doi:10.1111/gcb.13106 (2016).

$5 \quad$ Frontier, S. Etude de la décroissance des valeurs propres dans une analyse en composantes principales: comparaison avec le modèle du bâton brisé. Journal of Experimental Marine Biology and Ecology 25, 67-75 (1976).

6 Mackas, D. L. \& Beaugrand, G. Comparisons of zooplankton time series. Journal of Marine Systems 79, 286-304 (2010).

7 Mackas, D. L. et al. Changing zooplankton seasonality in a changing ocean: Comparing time series of zooplankton phenology. Progress in Oceanography 97-100, 31-62 (2012).

8 Kaiser, M. J. et al. Marine ecology: processes, systems and impacts., (Oxford University Press, 2005).

9 Morel, A. \& Prieur, L. Analysis of variations in ocean color. . Limnology and Oceanography 22, 709-722 (1977).

10 Reid, P. C. et al. The Continuous Plankton Recorder: concepts and history, from plankton indicator to undulating recorders. Progress in Oceanography 58, 117-173 (2003).

11 Batten, S. D. et al. CPR sampling: the technical background, materials, and methods, consistency and comparability. Progress in Oceanography 58, 193-215 (2003).

12 Goberville, E., Beaugrand, G. \& Edwards, M. Synchronous response of marine plankton ecosystems to climate in the Northeast Atlantic and the North Sea. Journal of Marine Systems, doi:10.1016/j.jmarsys.2013.05.008 (2013).

13 Cloern, J. E. \& Jassby, A. D. Drivers of change in estuarine-coastal ecosystems: discoveries from four decades of study in San Francisco Bay. Reviews of Geophysics 50, rg4001, doi:10.1029/2012RG000397 (2012).

14 Scheffer, M. Critical transitions in nature and society. (Princeton University Press, 2009).

15 Papworth, D. J., Marini, S. \& Conversi, A. A novel, unbiased analysis approach for investigating population dynamics: a case study on Calanus finmarchicus and its decline in the North Sea. . Plos One 11, e0158230, doi:10.1371/journal.pone.0158230 (2016).

16 Beaugrand, G. Theoretical basis for predicting climate-induced abrupt shifts in the oceans. Philosophical Tansactions of the Royal Society B: Biological Sciences 37020130264 , doi:10.1098/rstb.2013.0264 (2015).

17 S., C., Tadokoro, K., Sugisaki, H. \& Saino, T. Effects of decadal climate change on zooplankton over the last 50 years in the western subarctic North Pacific. . Global Change Biology 12, 907920, doi:10.1111/j.1365-2486.2006.01136.x (2006).

18 Di Lorenzo, E. et al. Synthesis of Pacific Ocean climate and ecosystems dynamics. Oceanography 26, 68-81 (2014).

19 Hare, S. R. \& Mantua, N. J. Empirical evidence for North Pacific regime shifts in 1977 and 1989. Progress in Oceanography 47, 103-145 (2000).

20 de Young, B. et al. Regime shifts in marine ecosystems: detection, prediction and management. Trends in Ecology and Evolution 23, 402-409 (2008). 
Powell, A. M. \& Xu, J. Decadal regime shift linkage between global marine fish landings and atmospheric planetary wave forcing. Earth System Dynamics 6, 125-146 (2015). Stenseth, N. C. et al. Ecological effects of climate fluctuations. Science 297, 1292-1296 (2002). Taylor, A. H., Allen, J. I. \& Clark, P. A. Extraction of a weak climatic signal by an ecosystem. Nature 416, 629-632 (2002). Beaugrand, G. Marine biodiversity, climatic variability and global change., (Routledge, 2015). Beaugrand, G. \& Kirby, R. R. How do marine species respond to climate change? Theories and observations. Annual Review of Marine Sciences 10, 169-197, doi:10.1146/121916-063304 (2018).

26 Beaugrand, G., Luczak, C., Goberville, E. \& Kirby, R. R. Marine biodiversity and the chessboard of life Plos One 13, e0194006, doi:https://doi.org/10.1371/journal.pone.0194006 (2018). Pörtner, H. O. \& Farrell, A. P. Physiology and climate change. Science 322, 690-692 (2008). Brown, J. H., Gillooly, J. F., Allen, A. P., Savage, V. M. \& West, G. B. Toward a metabolic theory of ecology. Ecology 85, 1771-1789 (2004).

29 Beaugrand, G., Reid, P. C., Ibañez, F., Lindley, J. A. \& Edwards, M. Reorganisation of North Atlantic marine copepod biodiversity and climate. Science 296, 1692-1694 (2002). Schmidt-Nielsen, K. Animal physiology: adaptation and environment. 4 edn, (Cambridge University Press, 1990).

31 Kirby, R. R. \& Beaugrand, G. Trophic amplification of climate warming. Proceedings of the Royal Society London B: Biological Sciences 276, 4095-4103 (2009).

32 Sarmiento, J. L. et al. Response of ocean ecosystems to climate warming. Global Biogeochemical Cycles 18, 1-23, doi:GB3003, doi: 10.1029/2003GB002134 (2004).

33 Beaugrand, G., Edwards, M. \& Legendre, L. Marine biodiversity, ecosystem functioning and the carbon cycles. Proceedings of the National Academy of Sciences of the USA 107, 1012010124 (2010).

34 Conversi, A., Peluso, T. \& Fonda-Umani, S. The Gulf of Trieste: a changing ecosystem. Journal of Geophysical Research 114, C03S90, doi:10.1029/2008JC004763 (2009).

35 Rutherford, S., D'Hondt, S. \& Prell, W. Environmental controls on the geographic distribution of zooplankton diversity. Nature 400, 749-753 (1999).

Rombouts, I. et al. Global latitudinal variations in marine copepod diversity and environmental factors. Proceedings of the Royal Society B 276, 3053-3062 (2009). Rombouts, I. et al. A multivariate approach to large-scale variation in marine planktonic copepod diversity and its environmental correlates. Limnology and Oceanography 55, 22192229 (2010).

38 Sunagawa, S. et al. Structure and function of the global ocean microbiome. Science 348, doi:10.1126/science.1261359 (2015).

39 Sverdrup, H. U. On conditions for the vernal blooming of phytoplankton. Journal du Conseil Permanent International pour l'Exploitation de la Mer 18, 287-295 (1953).

40 Behrenfeld, M. J. Abandoning Sverdrup's critical depth hypothesis on phytoplankton blooms. Ecology 91, 977-989 (2010).

41 Helaouët, P. \& Beaugrand, G. Macroecology of Calanus finmarchicus and C. helgolandicus in the North Atlantic Ocean and adjacent seas. Marine Ecology Progress Series 345, 147-165 (2007).

42 Goberville, E., Beaugrand, G., Sautour, B. \& Tréguer, P. Climate-driven changes in coastal marine systems of Western Europe. Marine Ecology Progress Series 408, 129:147 (2010).

43 Longhurst, A. Ecological geography of the sea. (Elsevier, 2007).

44 Orr, J. C. et al. Anthropogenic ocean acidification over the twenty-first century and its impact on calcifying organisms. Nature 437, 681-686 (2005).

45 Kroeker, K. J., Kordas, R. L., Crim, R. N. \& Singh, G. G. Meta-analysis reveals negative yet variable effects of ocean acidification on marine organisms. Ecology Letters 13, 1419-1434 (2010). 
Rothschild, B. J. \& Osborn, T. R. Small-scale turbulence and plankton contact rates. Journal of Plankton Research 10, 465-474 (1988).

47 Jolly, M. T. et al. Population genetics and hydrodynamic modeling of larval dispersal dissociate contemporary patterns of connectivity from historical expansion into European shelf seas in the polychaete Pectinaria koreni (Malmgren). Limnology and Oceanography 54, 2089-2106 (2009).

48 Nelson, G. C. et al. Anthropogenic Drivers of Ecosystem Change: an Overview. Ecology and Society 11, 29.

49 Papworth, D. J., Marini, S. \& Conversi, A. A novel, unbiased analysis approach for investigating population dynamics: a case study on Calanus finmarchicus and its decline in the North Sea. Plos One 11, e0158230, doi:10.1371/journal.pone.0158230 (2016).

50 Estes, J. A. et al. Trophic downgrading of Planet Earth. Science 333, 301-306 (2011).

51 Beaugrand, G., Brander, K. M., Lindley, J. A., Souissi, S. \& Reid, P. C. Plankton effect on cod recruitment in the North Sea. Nature 426, 661-664 (2003).

52 Reid, P. C., Borges, M. \& Svenden, E. A regime shift in the North Sea circa 1988 linked to changes in the North Sea horse mackerel fishery. Fisheries Research 50, 163-171 (2001).

53 Conversi, A. et al. A holistic view of marine regime shifts. Philosophical Transations of the Royal Society B 370, 20130279, doi:10.1098/rstb.2013.0279 (2015).

54 Beaugrand, G., Edwards, M., Brander, K., Luczak, C. \& Ibañez, F. Causes and projections of abrupt climate-driven ecosystem shifts in the North Atlantic. Ecology Letters 11, 1157-1168 (2008).

55 Bestelmeyer, B. T. et al. Analysis of abrupt transitions in ecological systems. . Ecosphere 2, 126 (2011).

56 Beaugrand, G. The North Sea regime shift: evidence, causes, mechanisms and consequences. Progress in Oceanography 60, 245-262 (2004).

57 Edwards, M. E., Johns, D. G., Leterme, S. C., Svendsen, E. \& Richardson, A. J. Regional climate change and harmful algal blooms in the northeast Atlantic. Limnology and Oceanography 51, 820-829 (2006).

58 Barnosky, A. D. et al. Approaching a state shift in Earth's biosphere. Nature 486, 52-58 (2012).

59 Moberg, F. \& Folke, C. Ecological goods and services of coral reef ecosystems. Ecological Economics 29, 215-233 (1999).

60 Mac Nally, R., Albano, C. \& Fleishman, E. A scrutiny of the evidence for pressure-induced state shifts in estuarine and nearshore ecosystems. Austral Ecology 39, 898-906 (2014).

61 Scheffer, M., Carpenter, S., Foley, J. A., Folke, C. \& Walker, B. Catastrophic shifts in ecosystems. Nature 413, 591-596 (2001).

62 Rodionov, S. N. A sequential algorithm for testing climate regime shift. Geophysical Research Letters 31, L09204, doi:10.1029/2004GL019448 (2004).

63 Rodionov, S. Use of prewhitening in climate regime shift detection. Geophysical Research Letters 33, L12707, doi:10.1029/2006GL025904 (2006).

64 Webster, R. Automatic soil-boundary location from transect data. Journal of the International Association of Mathematical Geology 5, 27-37 (1973).

65 Beaugrand, G. \& Reid, P. C. Long-term changes in phytoplankton, zooplankton and salmon linked to climate change. Global Change Biology 9, 801-817 (2003).

66 Mantua, N. J. Methods for detecting regime shifts in large marine ecosystems: a review with approaches applied to North Pacific data. Progress in Oceanography 60, 165-182 (2004).

67 Weijerman, M., Lindeboom, H. \& Zuur, A. F. Regime shifts in marine ecosystems of the North Sea and Wadden Sea. Marine Ecology Progress Series 298, 21-39 (2005).

68 Chen, J. \& Gupta, A. K. Parametric statistical change-point analysis. With applications to genetics, medicine and finance. 2 edn, 273 (MA: Birkhauser, 2012).

69 Hufty, A. Introduction à la climatologie. 542 (de Boeck, 2005). 
Di Lorenzo, E. \& Ohman, M. D. A double-integration hypothesis to explain ocean ecosystem response to climate forcing. Proceedings of the National Academy of Sciences of the United States of America 110, 2496-2499, doi:10.1073/pnas.1218022110 (2013).

71 Beaugrand, G. et al. Synchronous marine pelagic regime shifts in the Northern Hemisphere. Philosophical Tansactions of the Royal Society B: Biological Sciences 370, 20130272, doi:10.1098/rstb.2013.0272 (2015).

72 Cloern, J. E. et al. Biological communities in San Francisco Bay track large-scale climate forcing over the North Pacific. Geophysical Research Letters 37, L21602, doi:10.1029/2010GL044774 (2010).

73 Landry, M. R., Al-Mutairi, H., Selph, K. E., Christensen, S. \& Nunnery, S. Seasonal patterns of mesozooplankton abundance and biomass at Station ALOHA. Deep Sea Research Part II: Topical Studies in Oceanography 48, 2037-2061 (2001).

74 Atkinson, A., Siegel, V., Pakhomov, E. \& Rothery, P. Long-term decline in krill stock and increase in salps within the Southern Ocean. Nature 432, 100-1003 (2004).

75 Hays, G. C. \& Warner, A. J. Consistency of towing speed and sampling depth for the Continuous Plankton Recorder. Journal of the Marine Biological Association of the U. K. 73, 967-970 (1993).

76 Mollmann, C. et al. Reorganization of a large marine ecosystem due to atmospheric and anthropogenic pressure: a discontinuous regime shift in the Central Baltic Sea. . Global Change Biology 15, 1377-1393 (2009).

77 Gorsky, G. et al. Digital zooplankton image analysis using the ZooScan integrated system. Journal of Plankton Research 32, 285-303 (2010).

78 Giani, M. et al. Recent changes in the marine ecosystems of the northern Adriatic Sea. Estuarine, Coastal and Shelf Science 115, 1-13, doi:org/10.1016/j.ecss.2012.08.023 (2012).

79 Kane, J. Zooplankton abundance trends on Georges Bank, 1977-2004. ICES Journal of Marine Science 64, 909-919 (2007).

80 Koslow, J. A., R., G., Lara-Lopez, A. \& Watson, W. Impact of declining intermediate-water oxygen on deepwater fishes in the California Current. Marine Ecology Progress Series 436, 207-218 (2011).

81 Ohman, M. D. \& Smith, P. E. A comparison of zooplankton sampling methods in the CalCOFI time series. . California Cooperative Oceanic Fisheries Investigations Reports 36, 153-158 (1995).

82 Ruggerone, G. T., Peterman, R. M., Dorner, B. \& Myers, K. W. Magnitude and trends in abundance of hatchery and wild pink salmon, chum salmon, and sockeye salmon in the North Pacific Ocean. Marine and Coastal Fisheries: Dynamics, Management, and Ecosystem Science 2, 306-328, doi:10.1577/C09-054.1 (2010).

83 Myers, K. W., Klovach, N. V., Gritsenko, O. F., Urawa, S. \& Royer, T. C. Stock-specific distributions of Asian and North American salmon in the open ocean, interannual changes, and oceanographic conditions. North Pacific Anadromous Fish Commission 4, 159-177 (2007). Chiba, S., Tadokoro, K., Sugisaki, H. \& Saino, T. Effects of decadal climate change on zooplankton over the last 50 years in the western subarctic North Pacific. Global Change Biology 12, 907-920, doi:10.1111/j.1365-2486.2006.01136.x (2006). Atkinson, A. et al. KRILLBASE: a circumpolar database of Antarctic krill and salp numerical densities, 1926-2016. Earth Sytstem Science Data 9, 1-18 (2017). Atkinson, A. et al. Oceanic circumpolar habitats of Antarctic krill. Marine Ecology Progress Series 362, 1-23 (2008). Grinnell, J. The niche-relations of the California thrasher. Auk 34, 427-433 (1917). Elton, C. Animal ecology. (Sidgwick and Jackson, 1927). Hutchinson, G. E. An introduction to population ecology. (Yale University Press, 1978). Whittaker, R. H. Communities and ecosystems. 2 edn, 352 (Macmillan, 1975).

91 Chase, J. M. \& Leibold, M. A. Ecological niches: linking classical and contemporary approches. (The University of Chicago, 2003). 
92 Cheung, W. W. L., Lam, V. W. Y. \& Pauly, D. Modelling present and climate_shifted distribution of marine fishes and invertebrates. 72 (Fisheries Centre Research Reports, 2008).

93 Albouy, C. et al. Projected climate change and the changing biogeography of coastal Mediterranean fishes. Journal of Biogeography 40, 534-547, doi:10.1111/jbi.12013 (2012).

94 Lenoir, S., Beaugrand, G. \& Lecuyer, E. Modelled spatial distribution of marine fish and projected modifications in the North Atlantic Ocean. Global Change Biology 17, 115-129, doi:10.1111/j.1365-2486.2010.02229.x (2011).

95 Raybaud, V. et al. Decline in Kelp in West Europe and Climate. PLOS One 8, e66044, doi:10.1371/journal.pone.0066044 (2013).

96 Araujo, M. B. \& Guisan, A. Five (or so) challenges for species distribution modelling. Journal of Biogeography 33, 1677-1688 (2006).

97 Helaouët, P., Beaugrand, G. \& Reid, P. C. Macrophysiology of Calanus finmarchicus in the North Atlantic Ocean. Progress in Oceanography 91, 217-228, doi:10.1016/j.pocean.2010.11.003 (2011).

98 Brown, J. H. On the relationship between abundance and distribution of species. The American Naturalist 124, 255-279 (1984).

99 Beaugrand, G., Mackas, D. \& Goberville, E. Applying the concept of the ecological niche and a macroecological approach to understand how climate influences zooplankton: advantages, assumptions, limitations and requirements. Progress in Oceanography 111, 75-90, doi:10.1016/j.pocean.2012.11.002 (2013).

100 Beaugrand, G. \& Kirby, R. R. Quasi-deterministic responses of marine species to climate change. Climate Research 69, 117-128, doi:10.3354/cr01398 (2016).

101 Beaugrand, G., Goberville, E., Luczak, C. \& Kirby, R. R. Marine biological shifts and climate. Proceedings of the Royal Society B: Biological Sciences 281, 20133350, doi:10.1098/rspb.2013.3350 (2014).

102 Beaugrand, G. How Do Marine Pelagic Species Respond to Climate Change? Theories and Observations. Annual Reviews of Marine Science In preparation (2017).

103 Beaugrand, G., Edwards, M., Raybaud, V., Goberville, E. \& Kirby, R. R. Future vulnerability of marine biodiversity compared with contemporary and past changes. Nature Climate Change 5, 695-701, doi:10.1038/NCLIMATE2650 (2015).

104 Gause, G. F. The struggle for coexistence. (MD: Williams and Wilkins, 1934).

105 Beaugrand, G., Rombouts, I. \& Kirby, R. R. Towards an understanding of the pattern of biodiversity in the oceans. Global Ecology and Biogeography 22, 440-449 (2013).

106 Alheit, J. \& Hagen, E. Long-term forcing of European herring and sardine populations. Fisheries Oceanography 6, 130-139 (1997).

107 Ruggerone, G. T., Peterman, R. M., Dorner, B., Myers, K. W. \& Mantua, N. Abundance of adult hatchery and wild salmon by region of the North Pacific. Report No. SAFS-UW 1001, Seattle, (Seattle, 2010). 


\section{Supplementary Figure 4}

\section{Percentage of species' shift into a community}

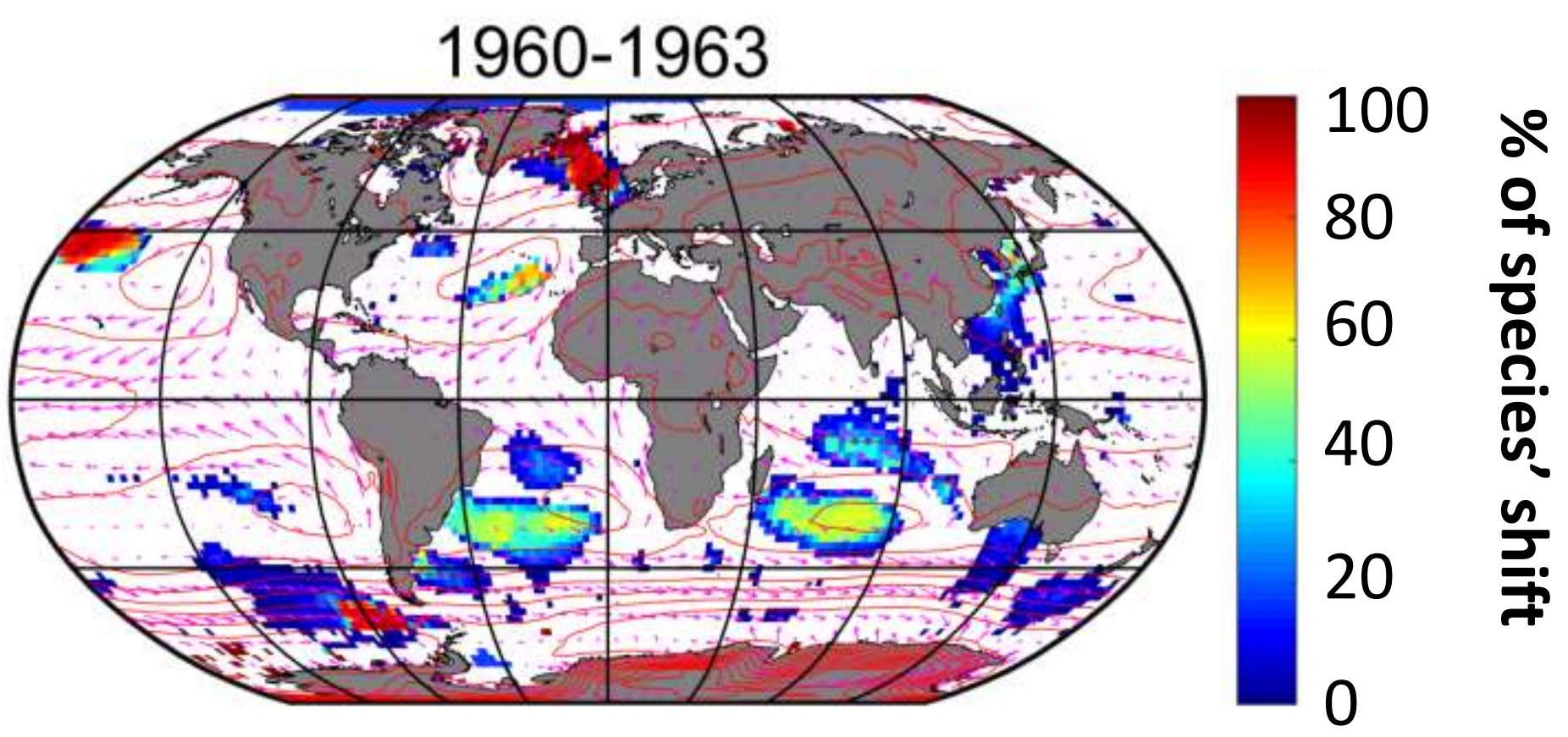

Pink arrow: direction and strengh of mean annual wind (1960-2015) Red line: isobar based on annual SLP for the period 1960-2015 White area: no significant species' shift (threshold $>3$ ) 


\section{Percentage of species' shift into a community}

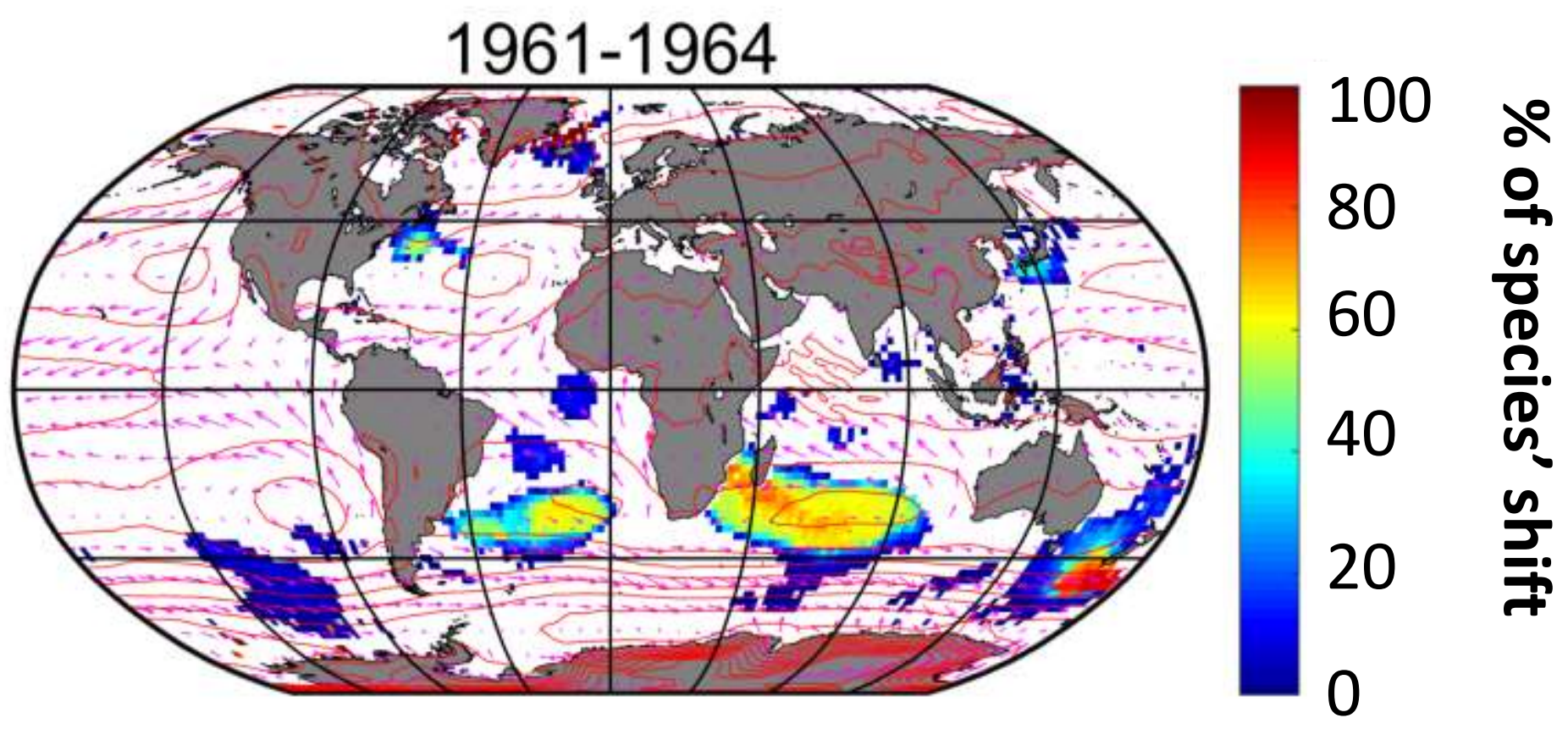

Pink arrow: direction and strengh of mean annual wind (1960-2015) Red line: isobar based on annual SLP for the period 1960-2015 White area: no significant species' shift (threshold $>3$ ) 


\section{Percentage of species' shift into a community}

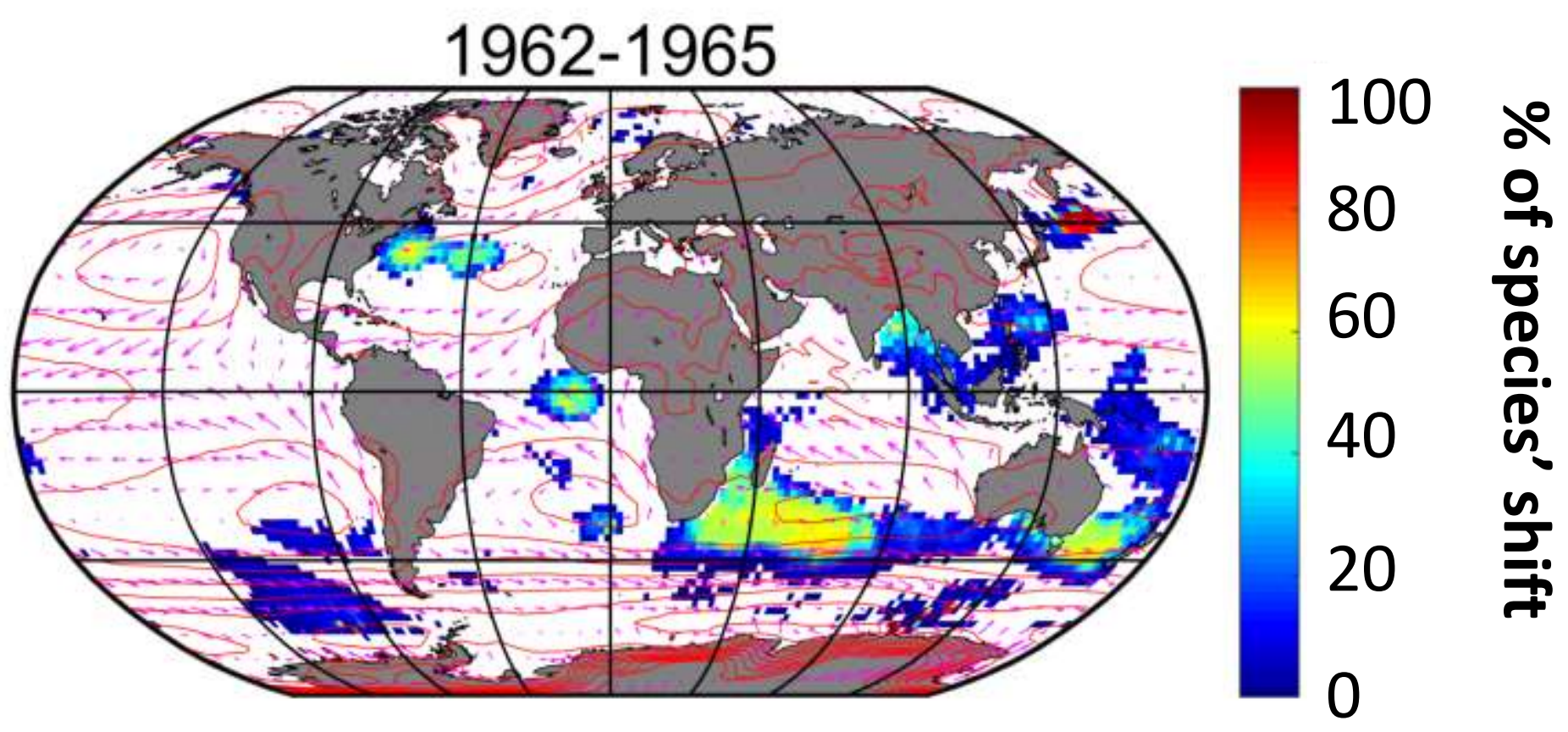

Pink arrow: direction and strengh of mean annual wind (1960-2015) Red line: isobar based on annual SLP for the period 1960-2015 White area: no significant species' shift (threshold $>3$ ) 


\section{Percentage of species' shift into a community}

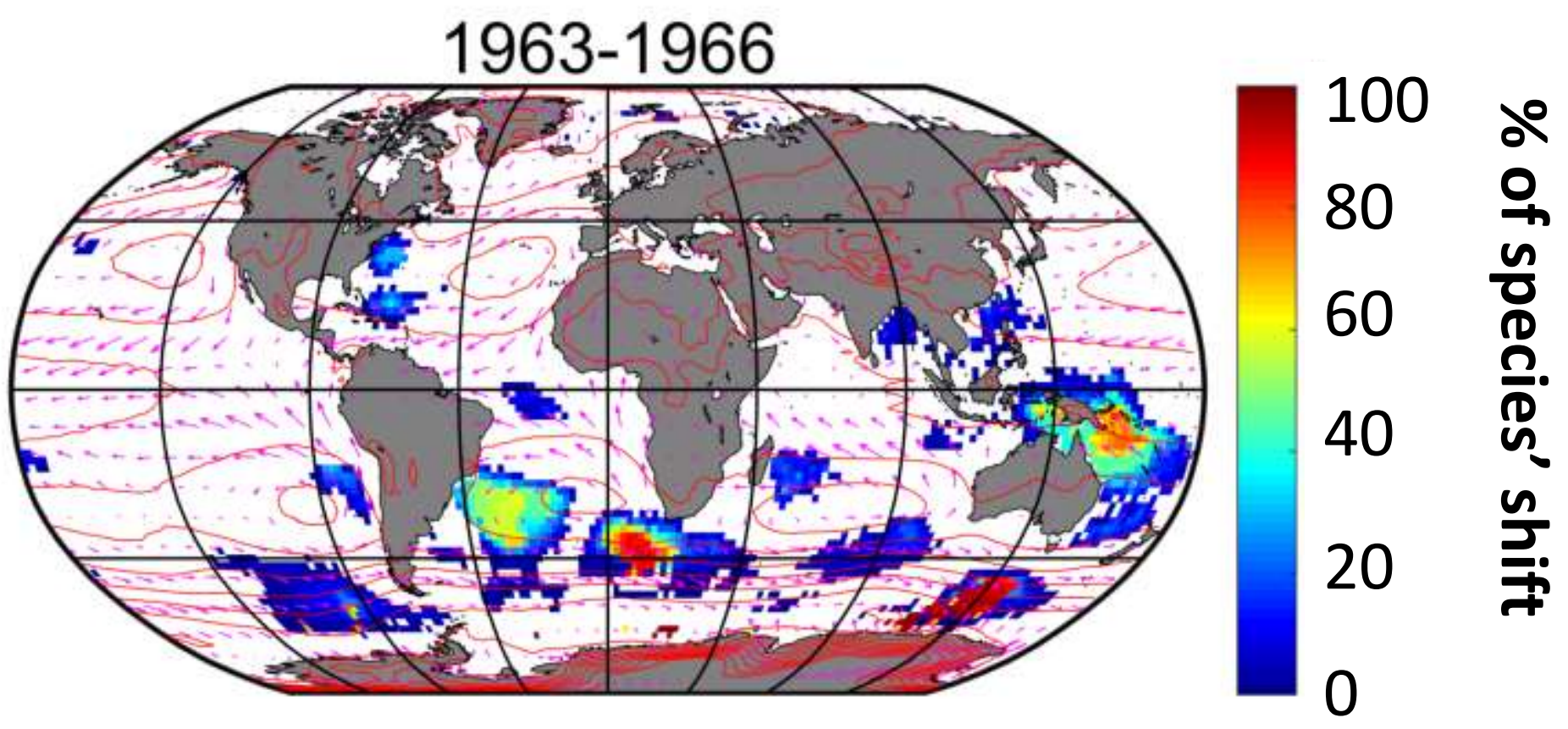

Pink arrow: direction and strengh of mean annual wind (1960-2015) Red line: isobar based on annual SLP for the period 1960-2015 White area: no significant species' shift (threshold $>3$ ) 


\section{Percentage of species' shift into a community}

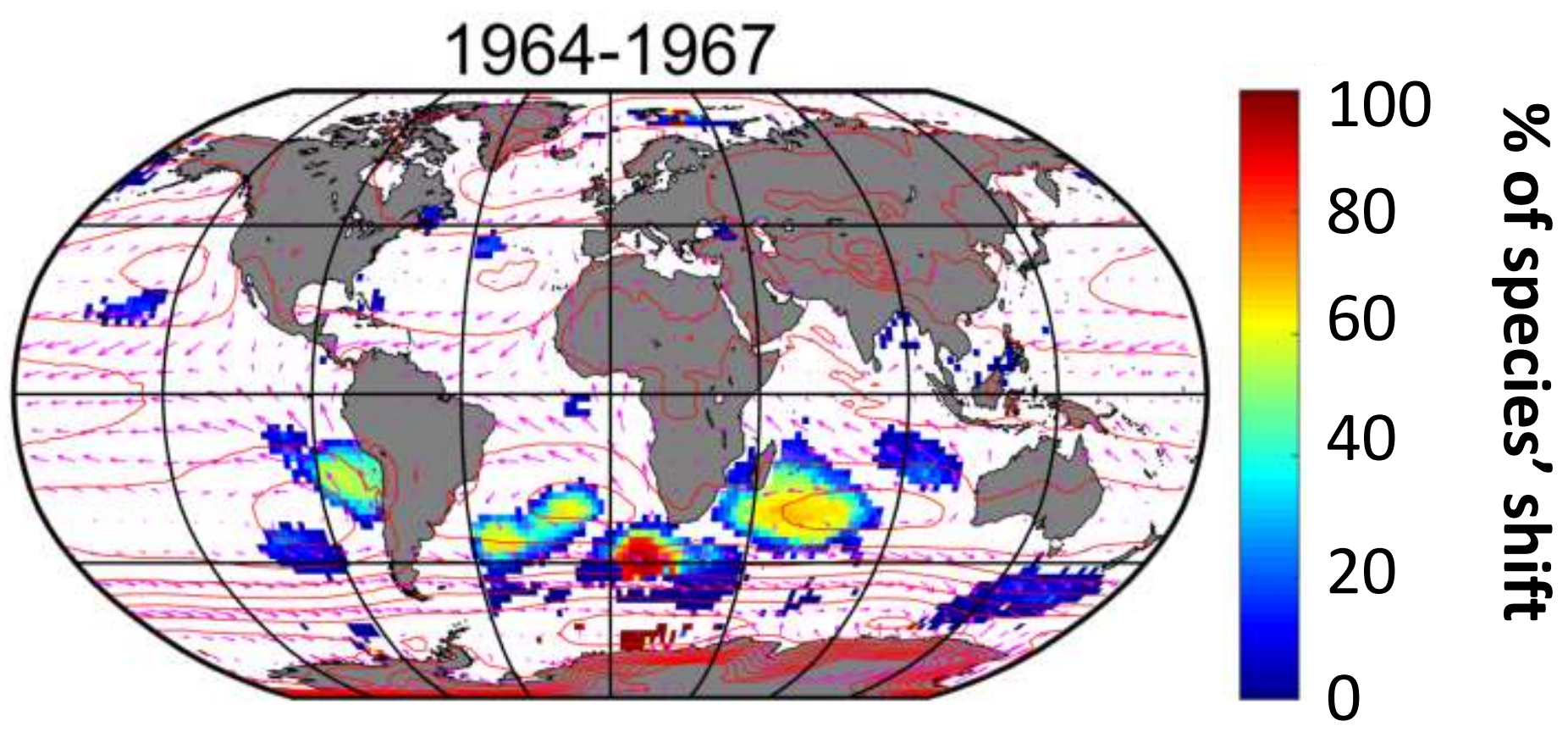

Pink arrow: direction and strengh of mean annual wind (1960-2015) Red line: isobar based on annual SLP for the period 1960-2015 White area: no significant species' shift (threshold $>3$ ) 


\section{Percentage of species' shift into a community}

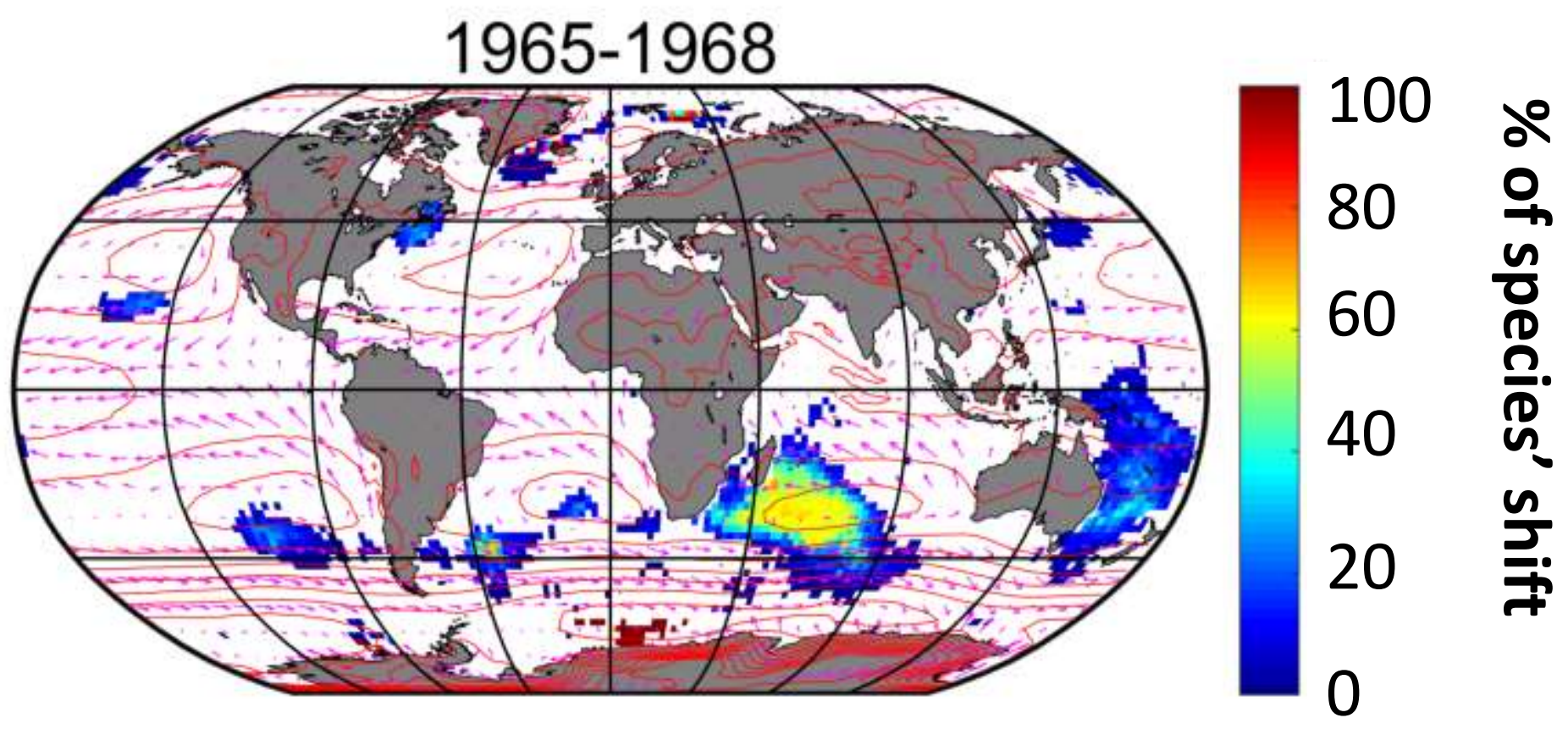

Pink arrow: direction and strengh of mean annual wind (1960-2015) Red line: isobar based on annual SLP for the period 1960-2015 White area: no significant species' shift (threshold $>3$ ) 


\section{Percentage of species' shift into a community}

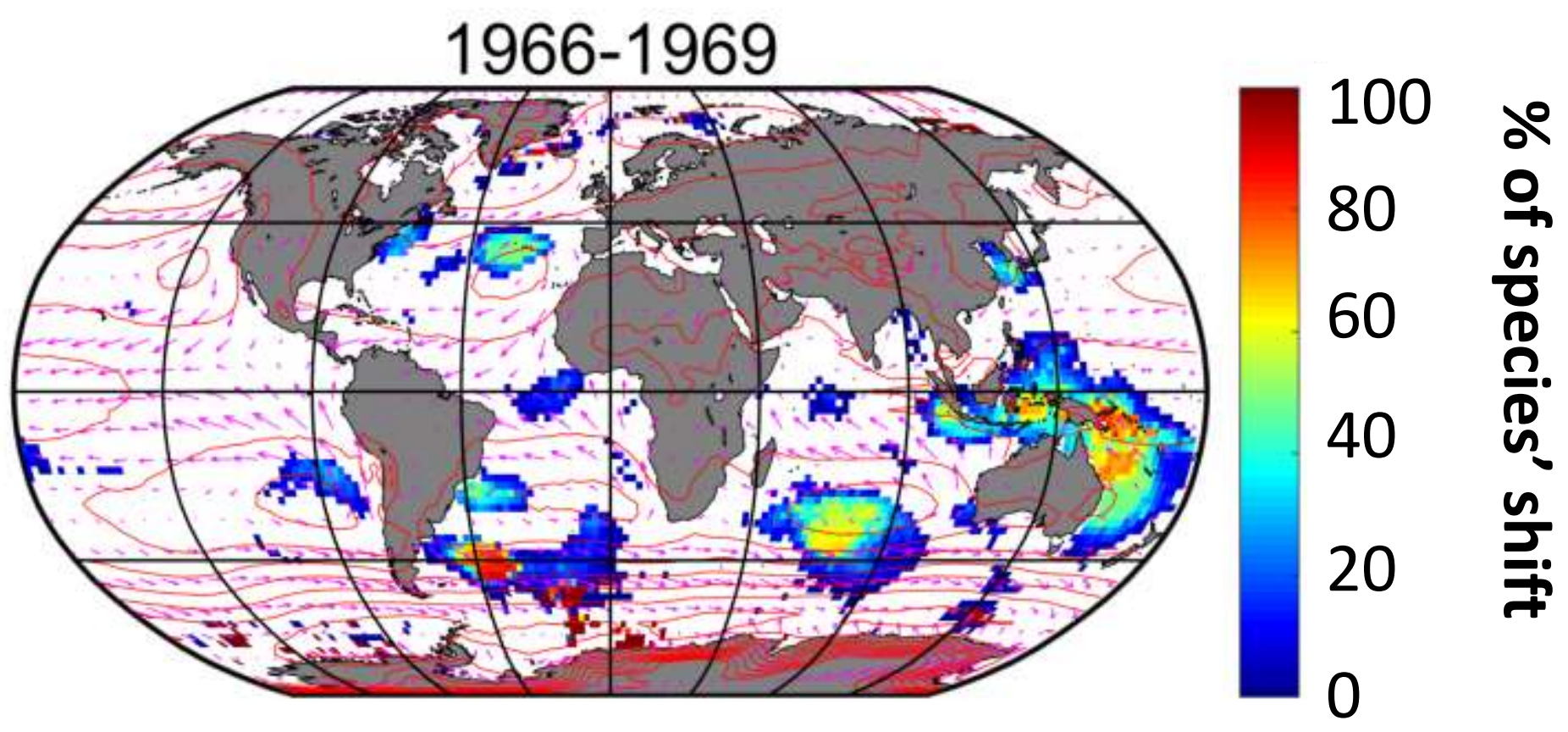

Pink arrow: direction and strengh of mean annual wind (1960-2015) Red line: isobar based on annual SLP for the period 1960-2015 White area: no significant species' shift (threshold $>3$ ) 


\section{Percentage of species' shift into a community}

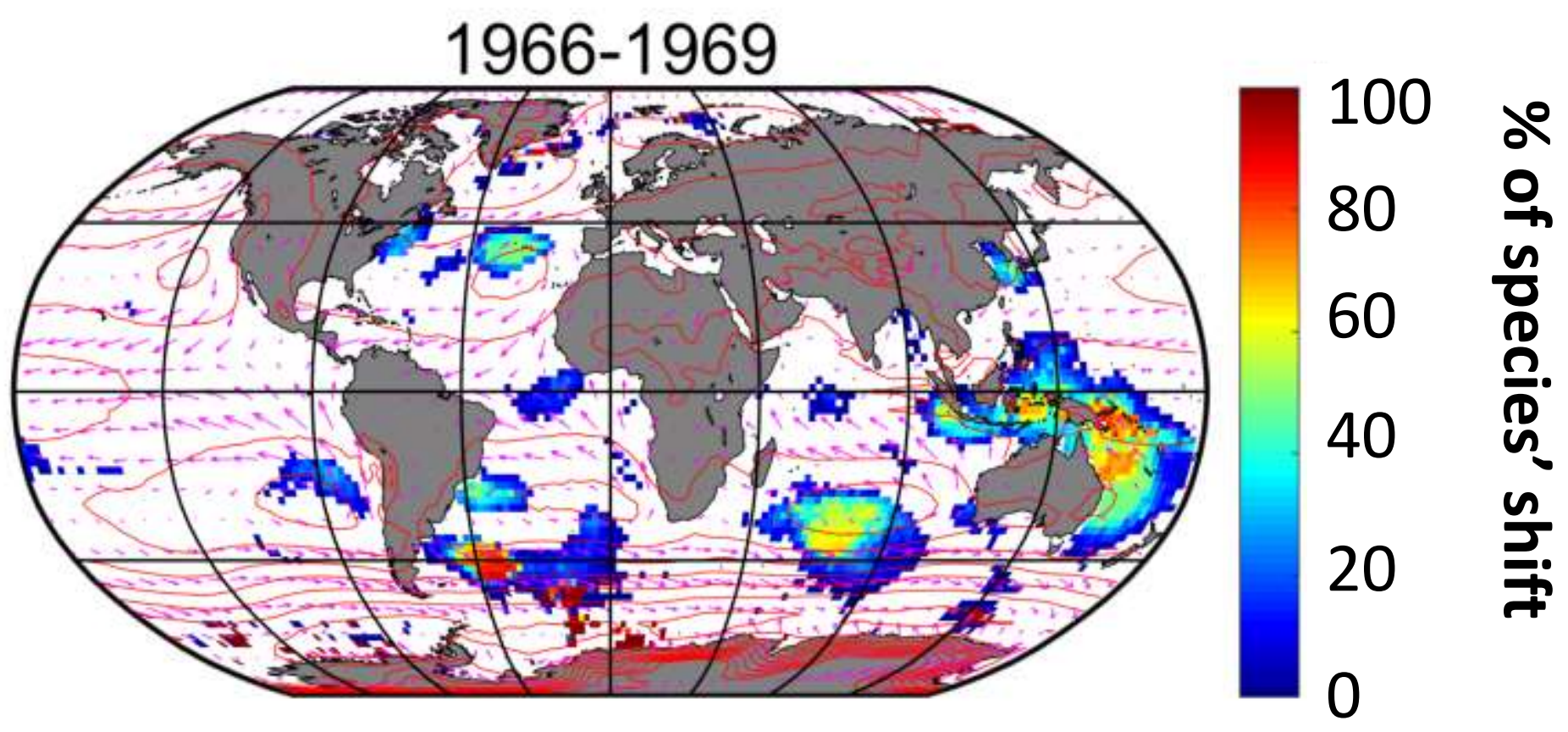

Pink arrow: direction and strengh of mean annual wind (1960-2015) Red line: isobar based on annual SLP for the period 1960-2015 White area: no significant species' shift (threshold $>3$ ) 


\section{Percentage of species' shift into a community}

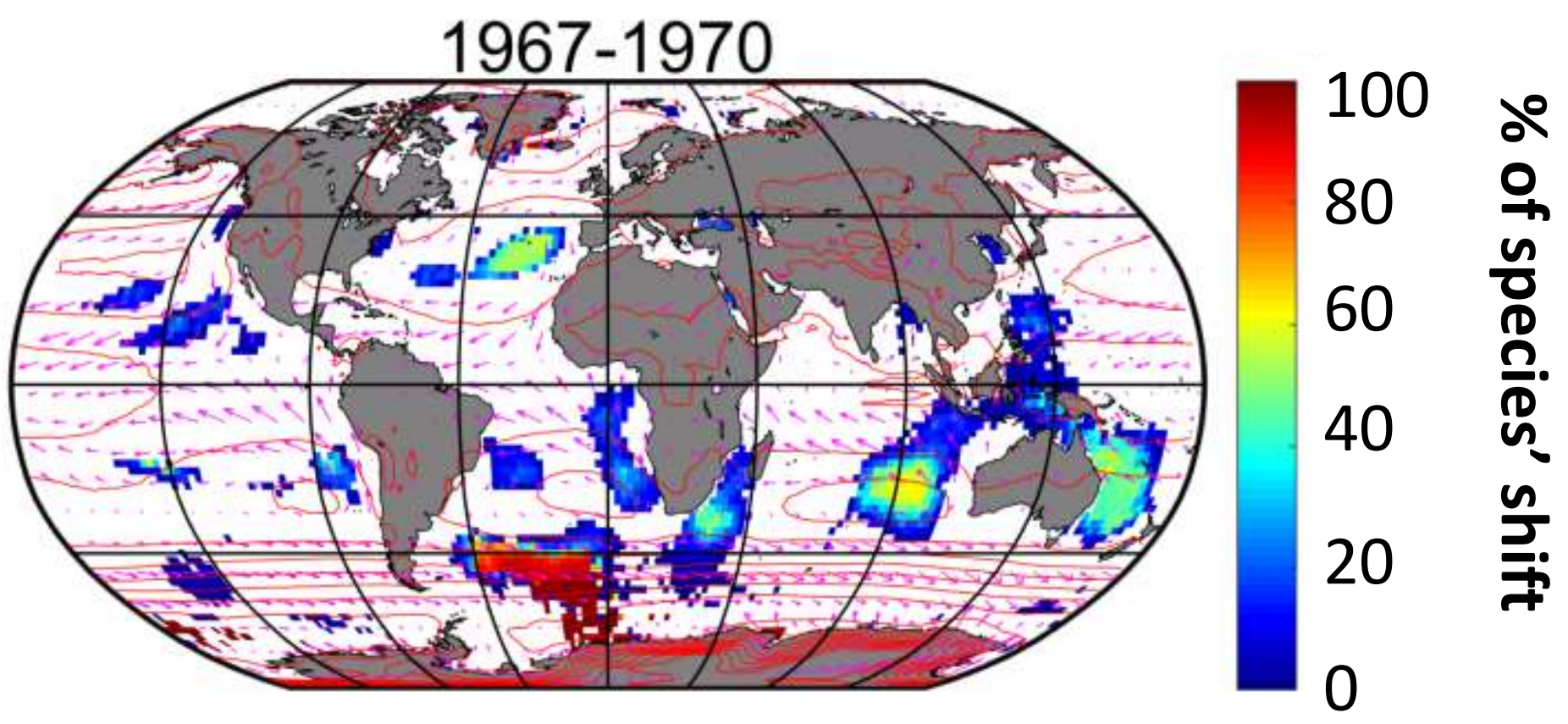

Pink arrow: direction and strengh of mean annual wind (1960-2015) Red line: isobar based on annual SLP for the period 1960-2015 White area: no significant species' shift (threshold $>3$ ) 


\section{Percentage of species' shift into a community}

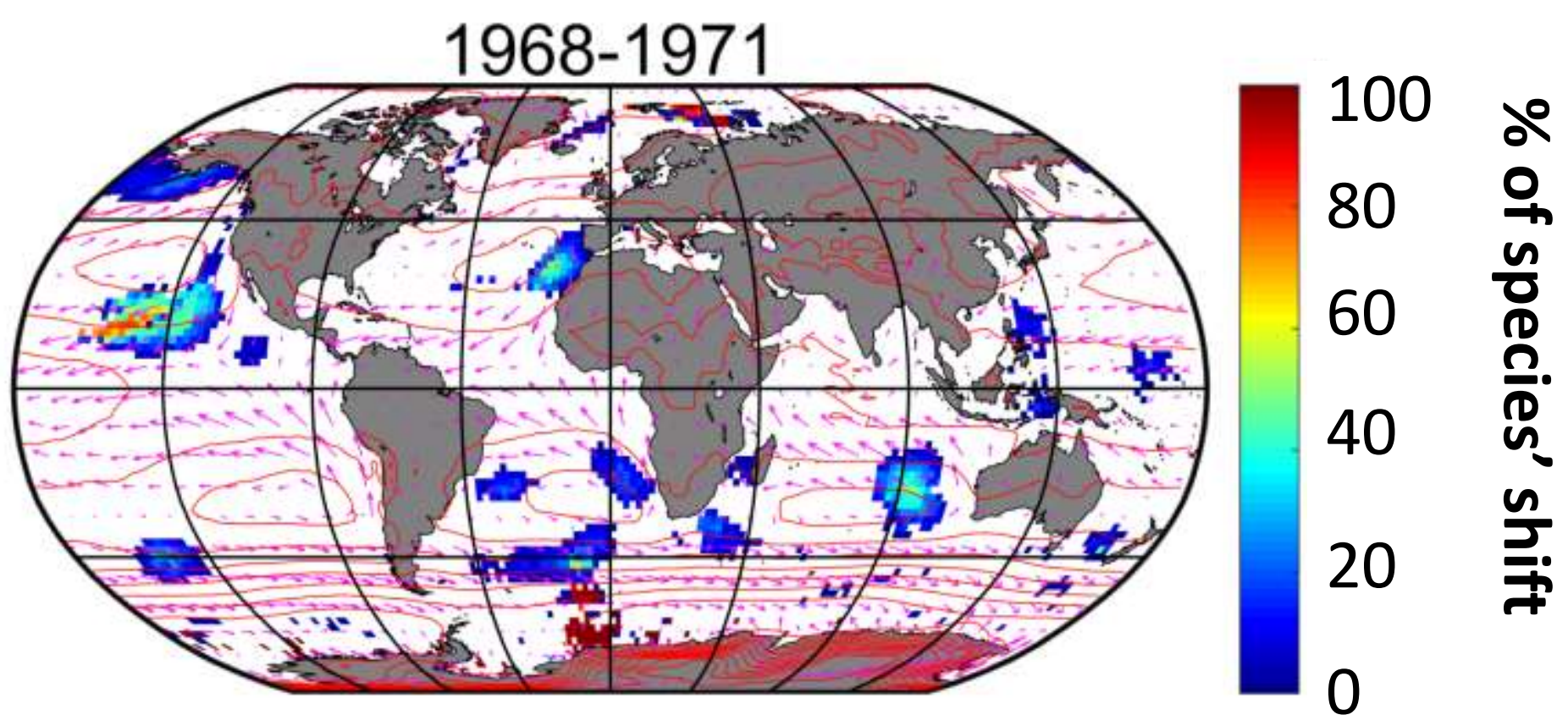

Pink arrow: direction and strengh of mean annual wind (1960-2015) Red line: isobar based on annual SLP for the period 1960-2015 White area: no significant species' shift (threshold $>3$ ) 


\section{Percentage of species' shift into a community}

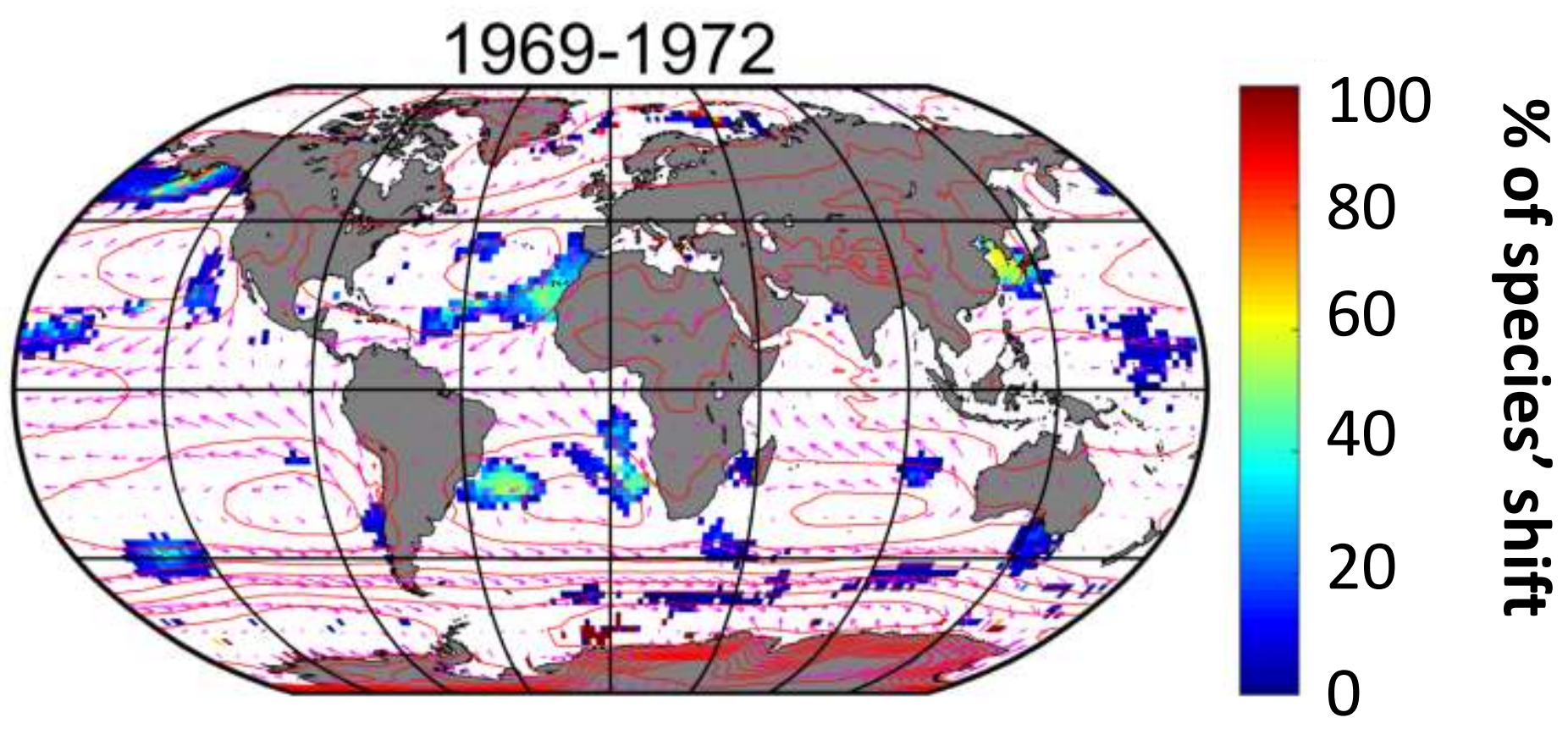

Pink arrow: direction and strengh of mean annual wind (1960-2015) Red line: isobar based on annual SLP for the period 1960-2015 White area: no significant species' shift (threshold $>3$ ) 


\section{Percentage of species' shift into a community}

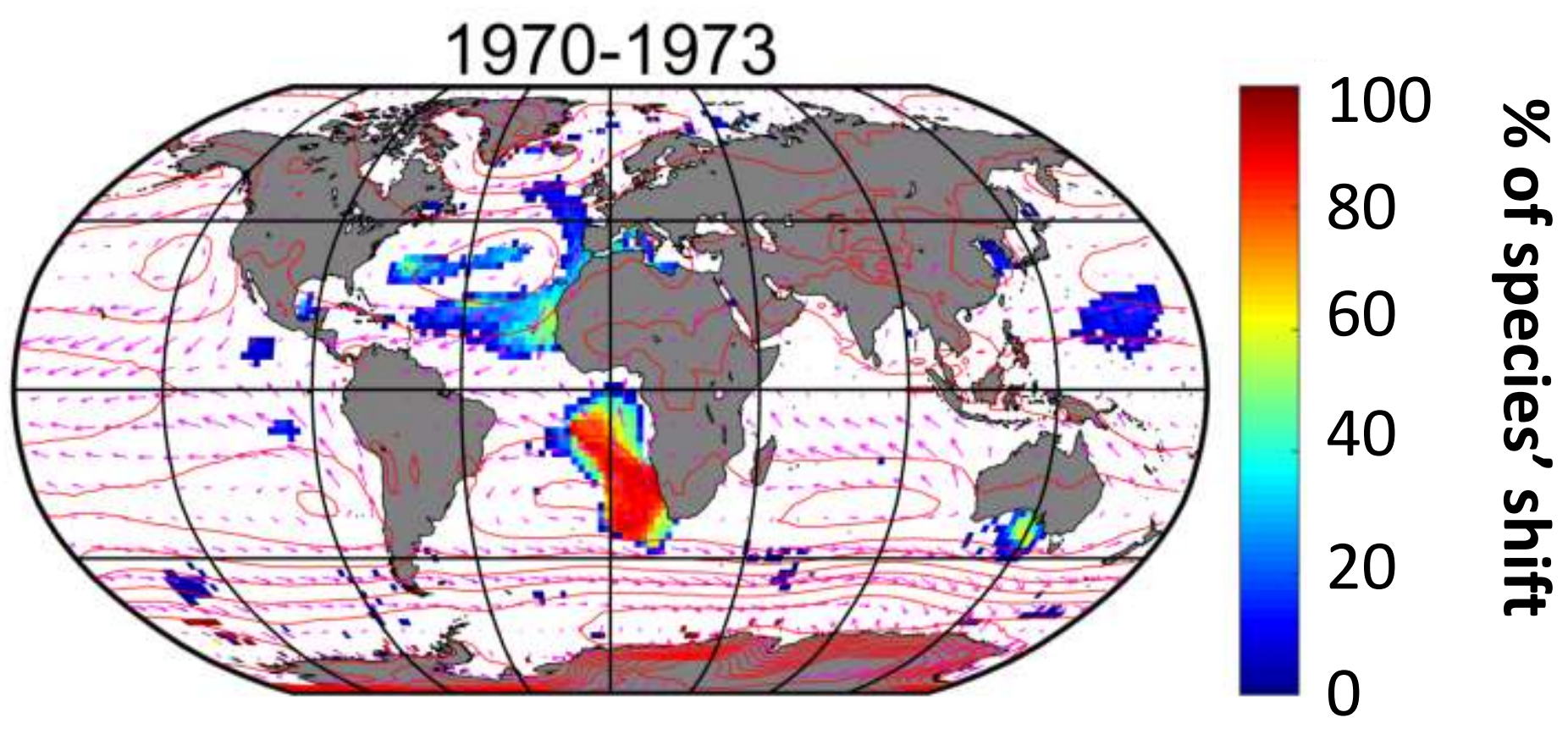

Pink arrow: direction and strengh of mean annual wind (1960-2015) Red line: isobar based on annual SLP for the period 1960-2015 White area: no significant species' shift (threshold $>3$ ) 


\section{Percentage of species' shift into a community}

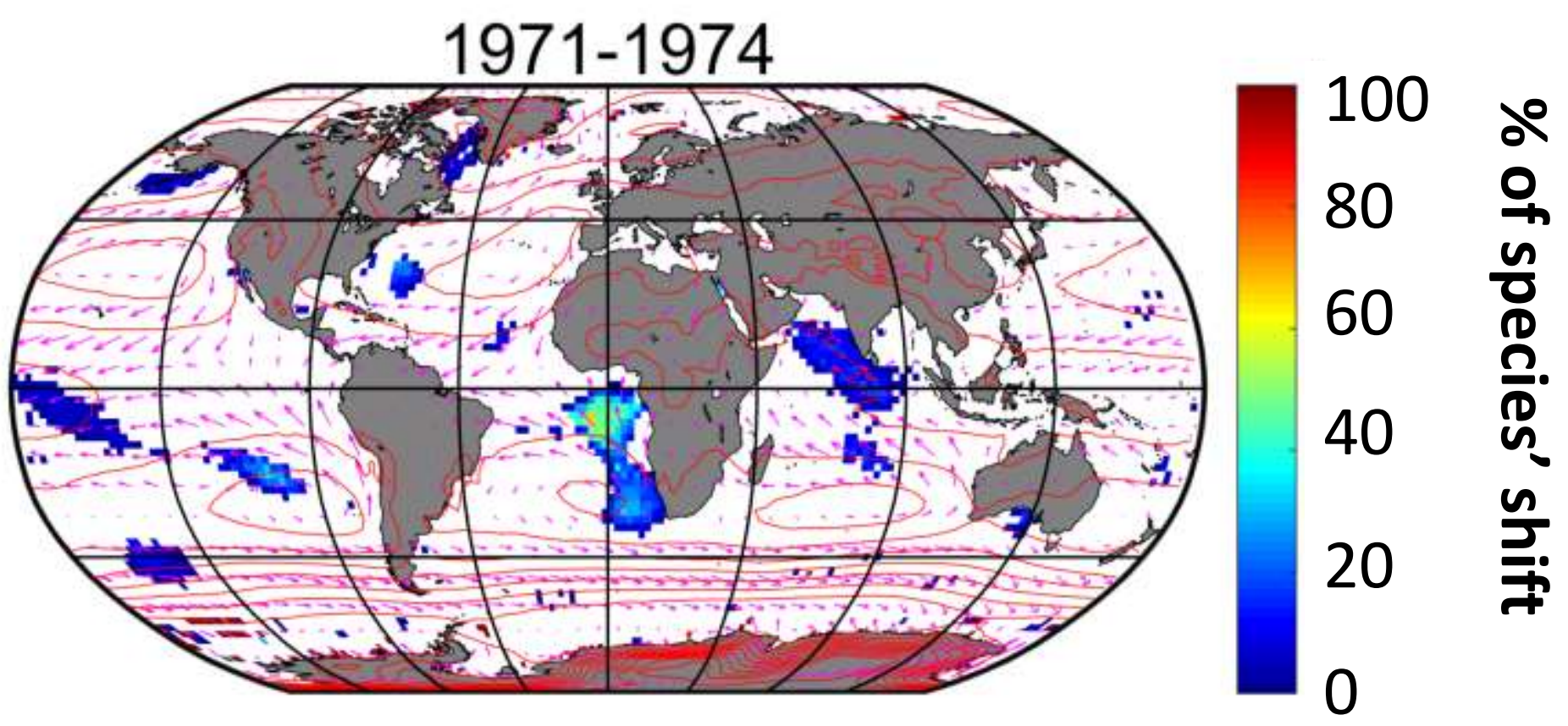

Pink arrow: direction and strengh of mean annual wind (1960-2015) Red line: isobar based on annual SLP for the period 1960-2015 White area: no significant species' shift (threshold $>3$ ) 


\section{Percentage of species' shift into a community}

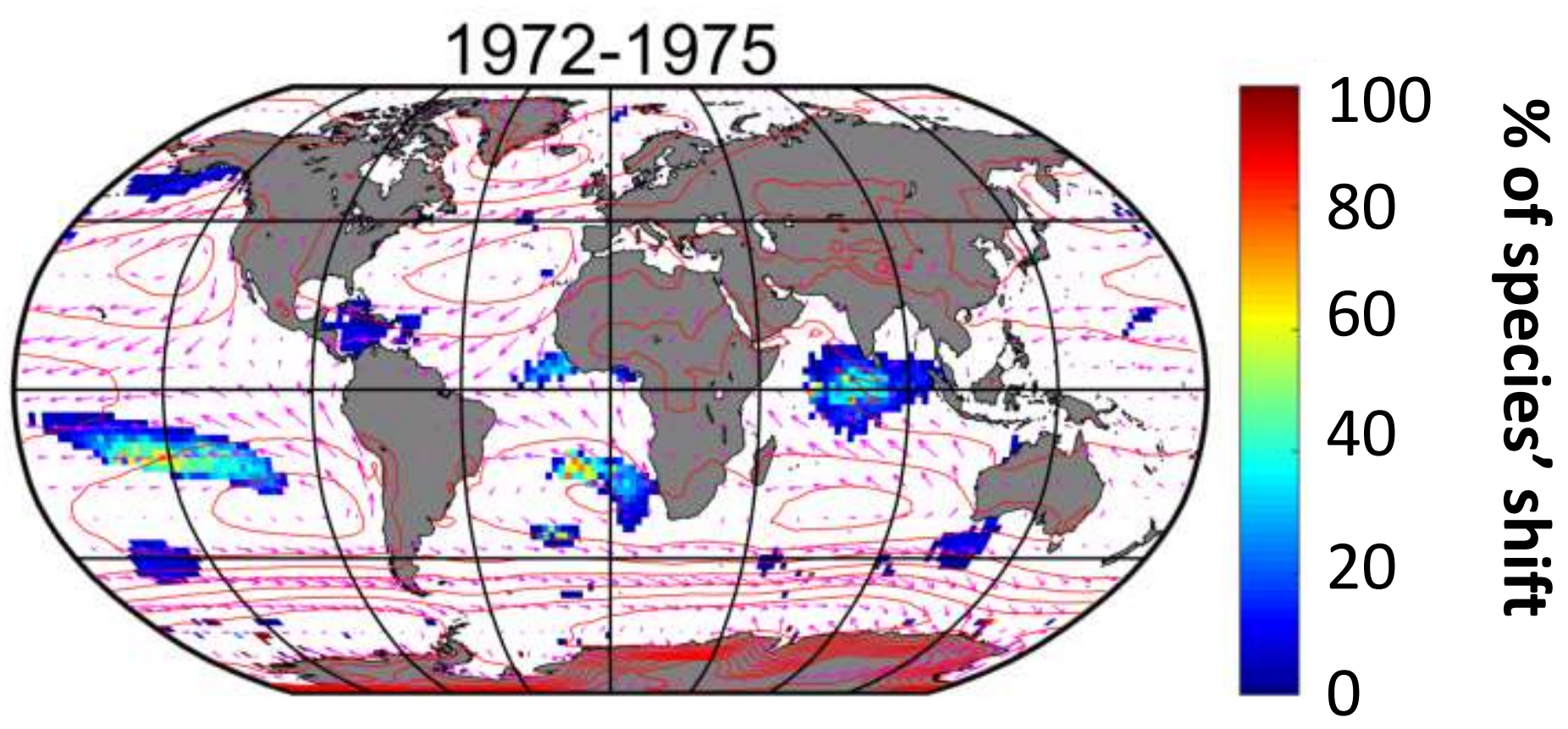

Pink arrow: direction and strengh of mean annual wind (1960-2015) Red line: isobar based on annual SLP for the period 1960-2015 White area: no significant species' shift (threshold $>3$ ) 


\section{Percentage of species' shift into a community}

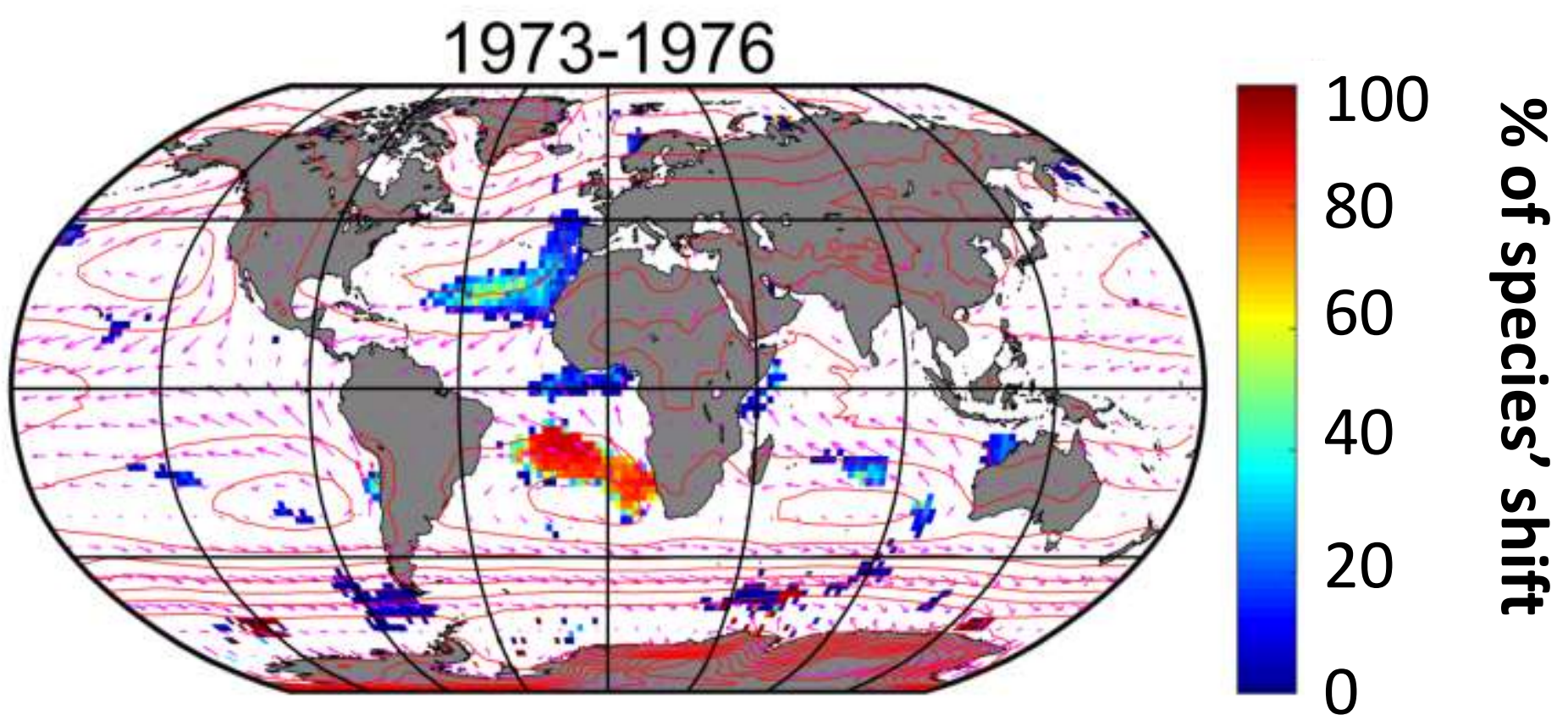

Pink arrow: direction and strengh of mean annual wind (1960-2015) Red line: isobar based on annual SLP for the period 1960-2015 White area: no significant species' shift (threshold $>3$ ) 


\section{Percentage of species' shift into a community}

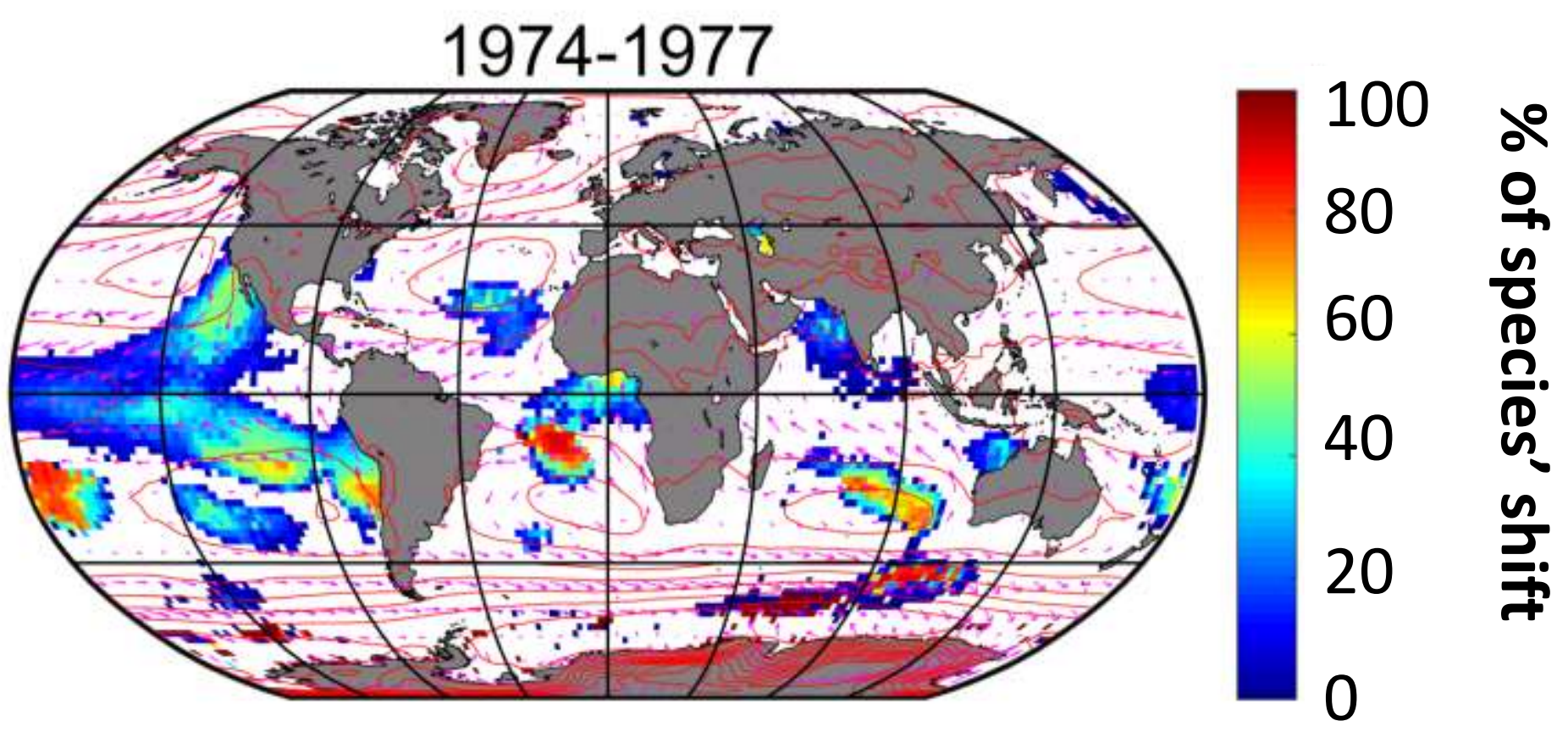

Pink arrow: direction and strengh of mean annual wind (1960-2015) Red line: isobar based on annual SLP for the period 1960-2015 White area: no significant species' shift (threshold $>3$ ) 


\section{Percentage of species' shift into a community}

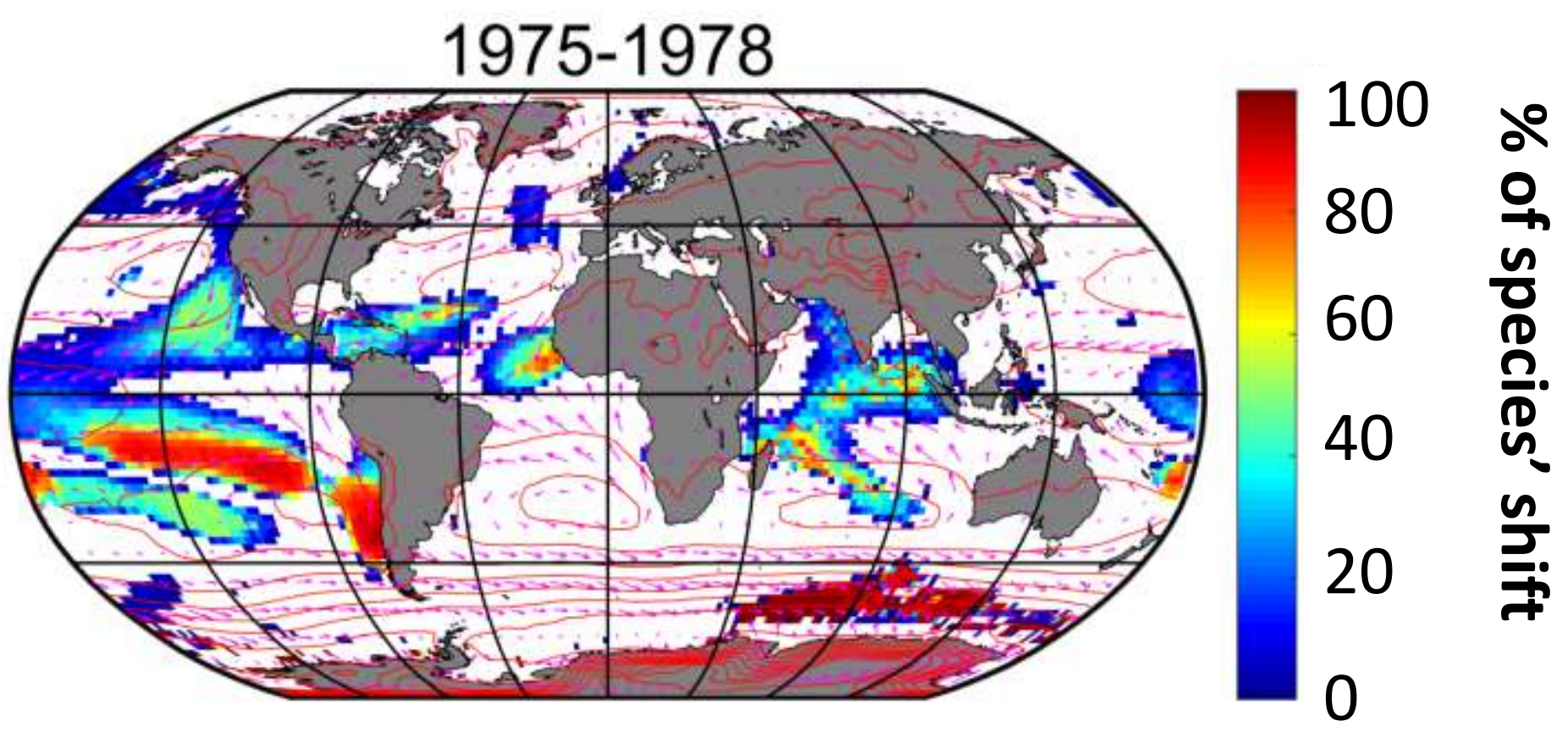

Pink arrow: direction and strengh of mean annual wind (1960-2015) Red line: isobar based on annual SLP for the period 1960-2015 White area: no significant species' shift (threshold $>3$ ) 


\section{Percentage of species' shift into a community}

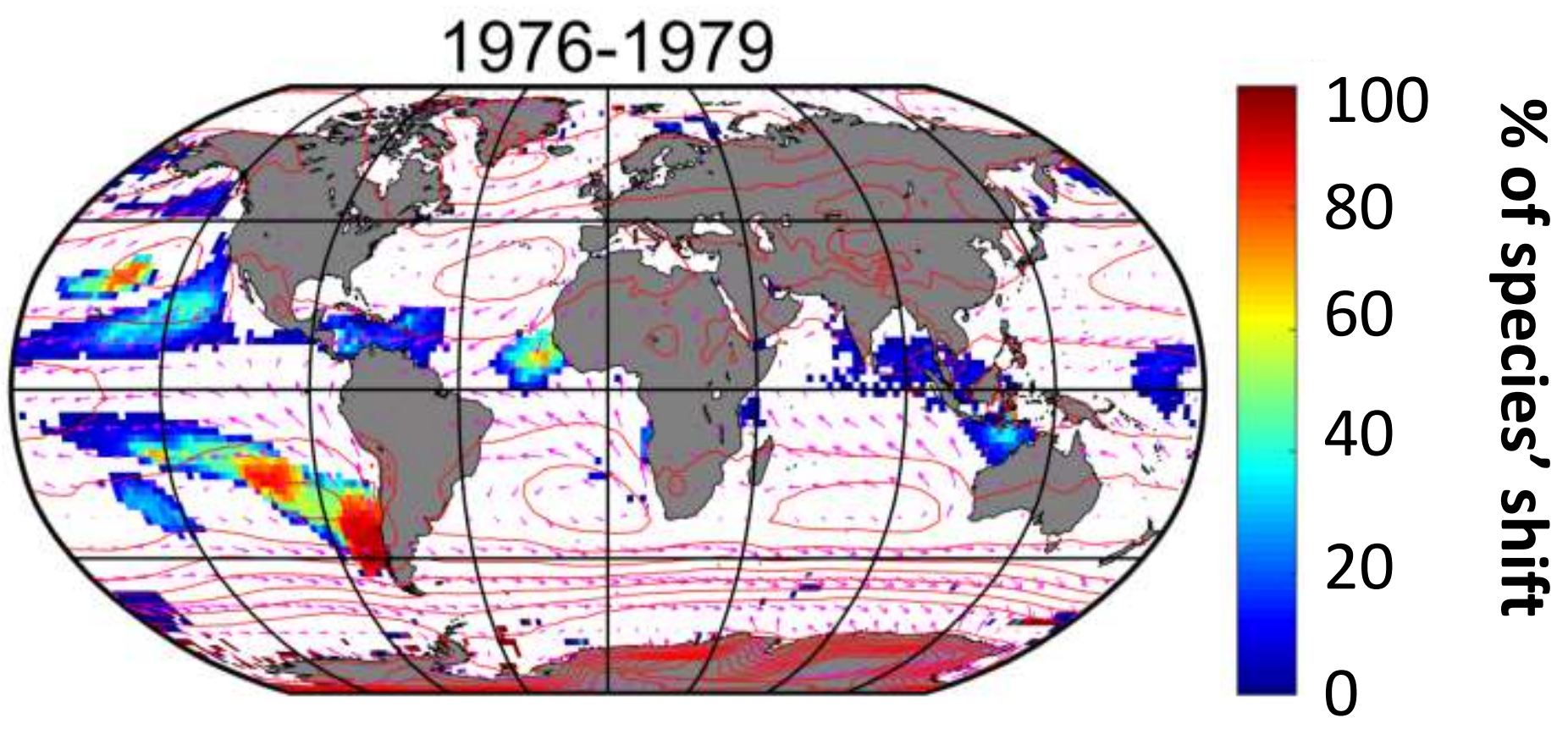

Pink arrow: direction and strengh of mean annual wind (1960-2015) Red line: isobar based on annual SLP for the period 1960-2015 White area: no significant species' shift (threshold $>3$ ) 


\section{Percentage of species' shift into a community}

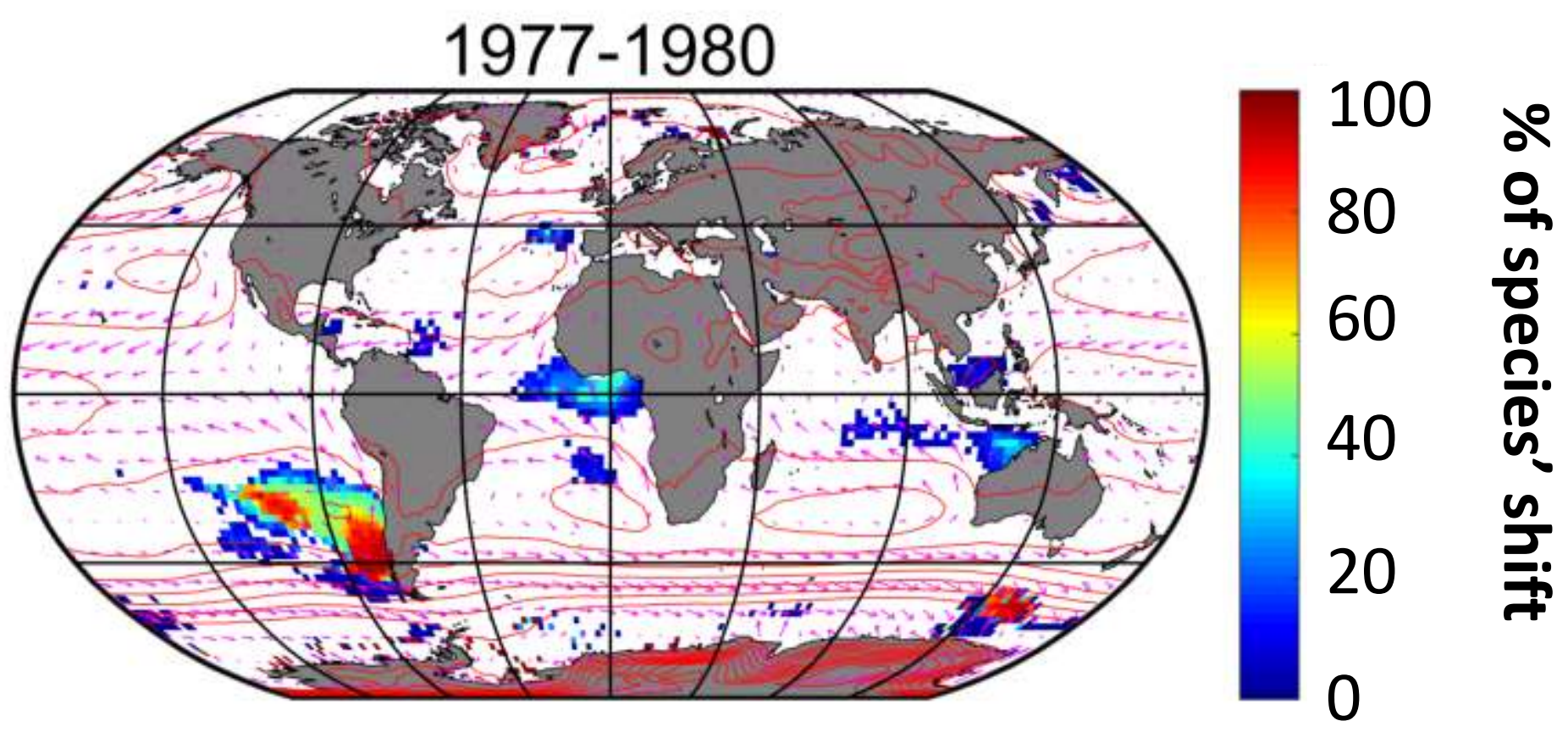

Pink arrow: direction and strengh of mean annual wind (1960-2015) Red line: isobar based on annual SLP for the period 1960-2015 White area: no significant species' shift (threshold $>3$ ) 


\section{Percentage of species' shift into a community}

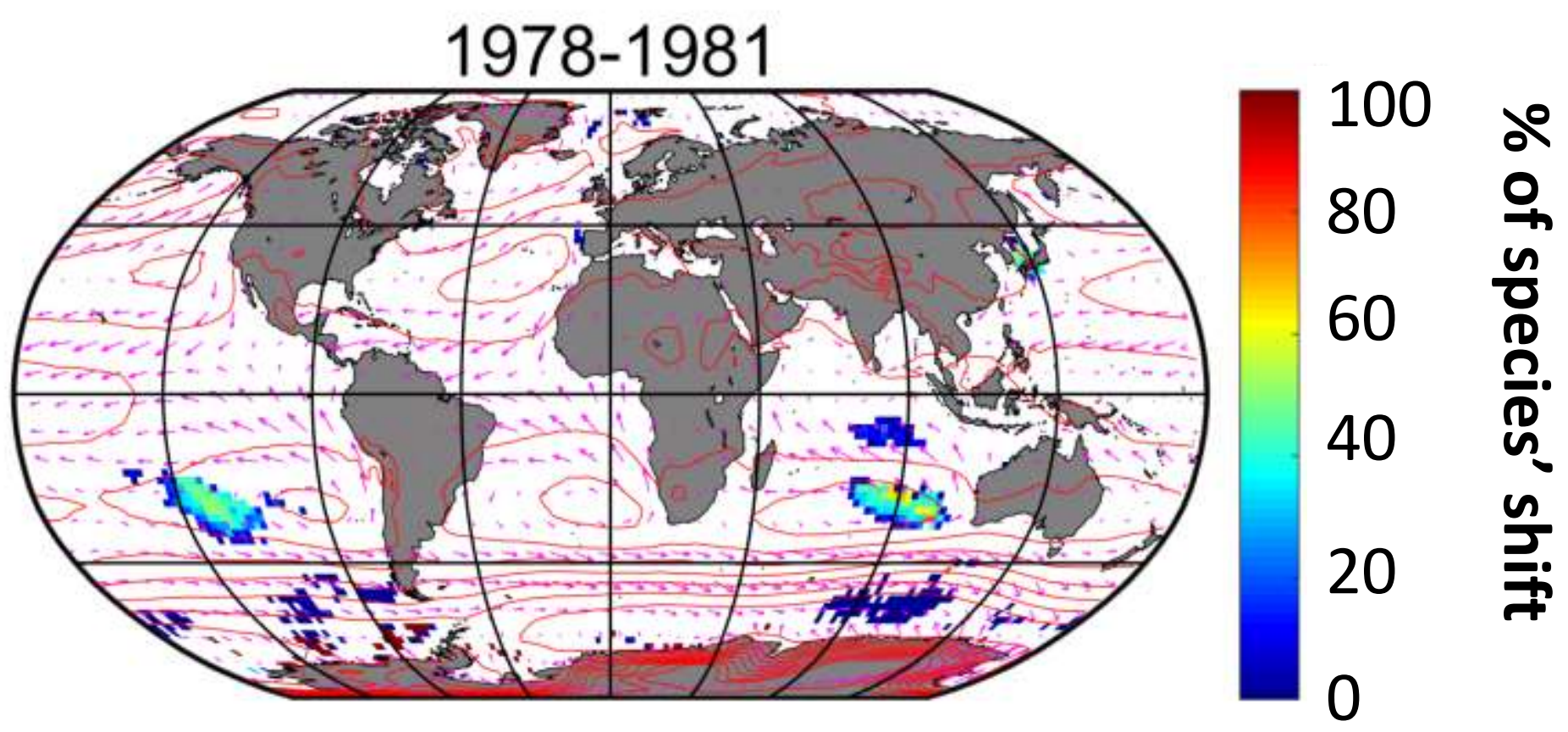

Pink arrow: direction and strengh of mean annual wind (1960-2015) Red line: isobar based on annual SLP for the period 1960-2015 White area: no significant species' shift (threshold $>3$ ) 


\section{Percentage of species' shift into a community}

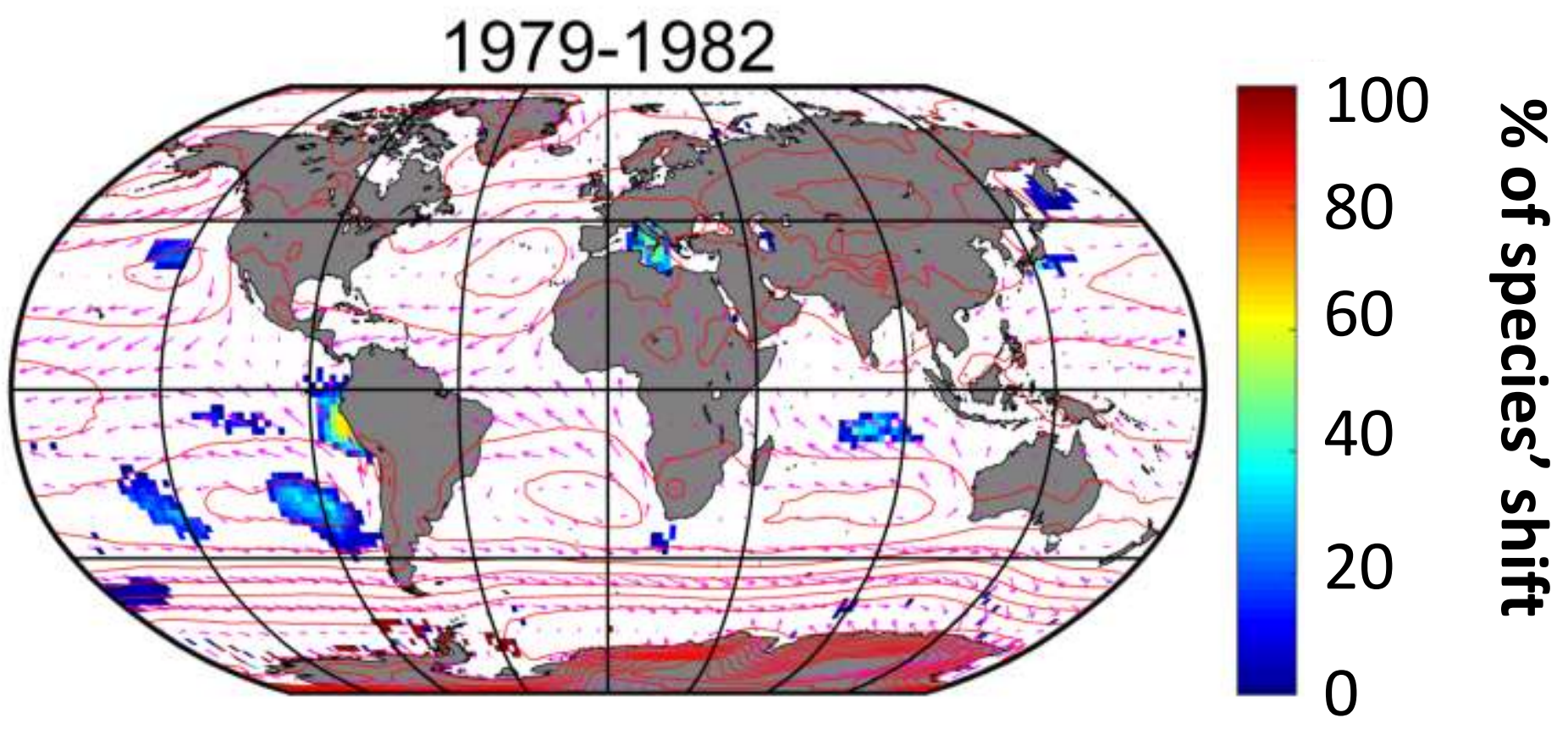

Pink arrow: direction and strengh of mean annual wind (1960-2015) Red line: isobar based on annual SLP for the period 1960-2015 White area: no significant species' shift (threshold $>3$ ) 


\section{Percentage of species' shift into a community}

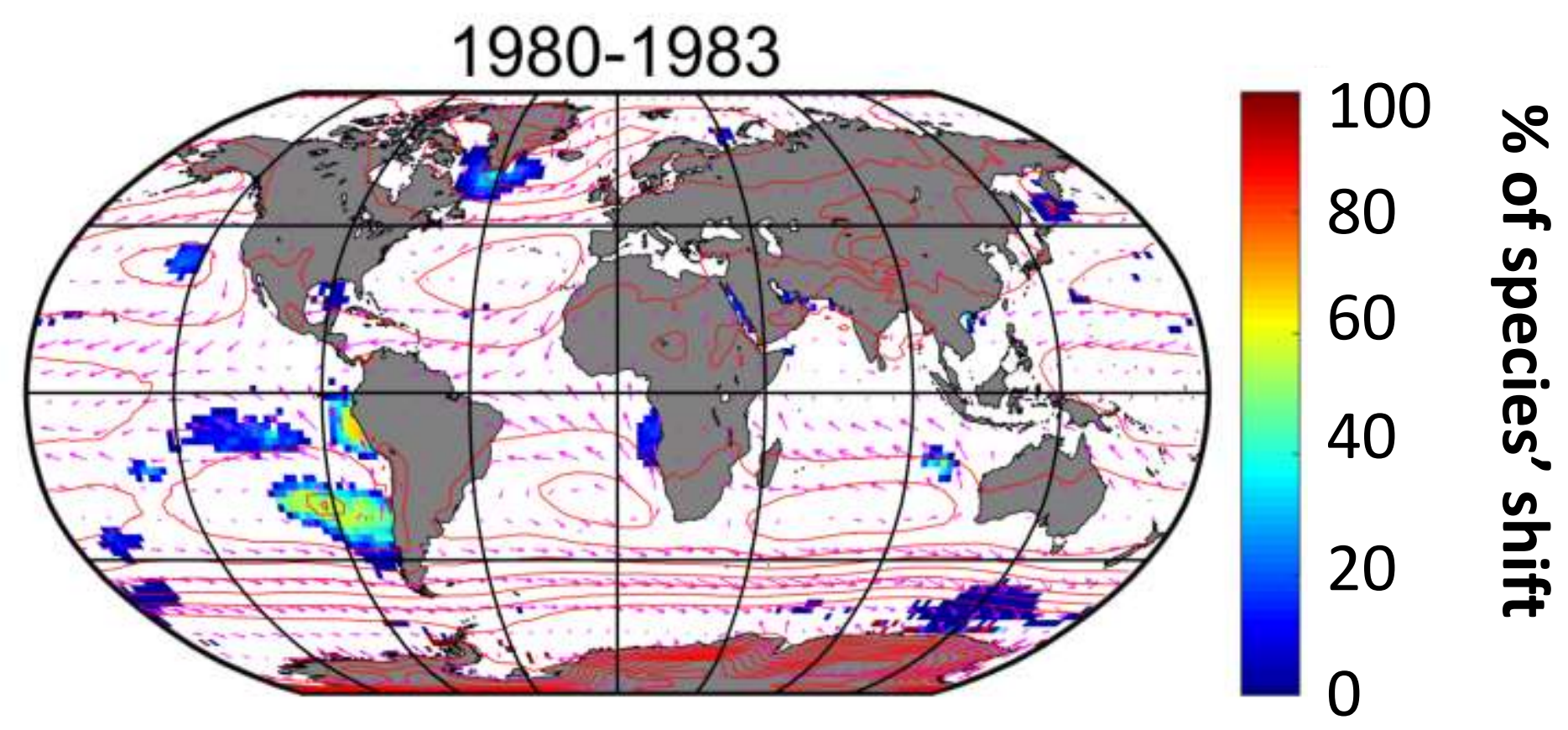

Pink arrow: direction and strengh of mean annual wind (1960-2015) Red line: isobar based on annual SLP for the period 1960-2015 White area: no significant species' shift (threshold $>3$ ) 


\section{Percentage of species' shift into a community}

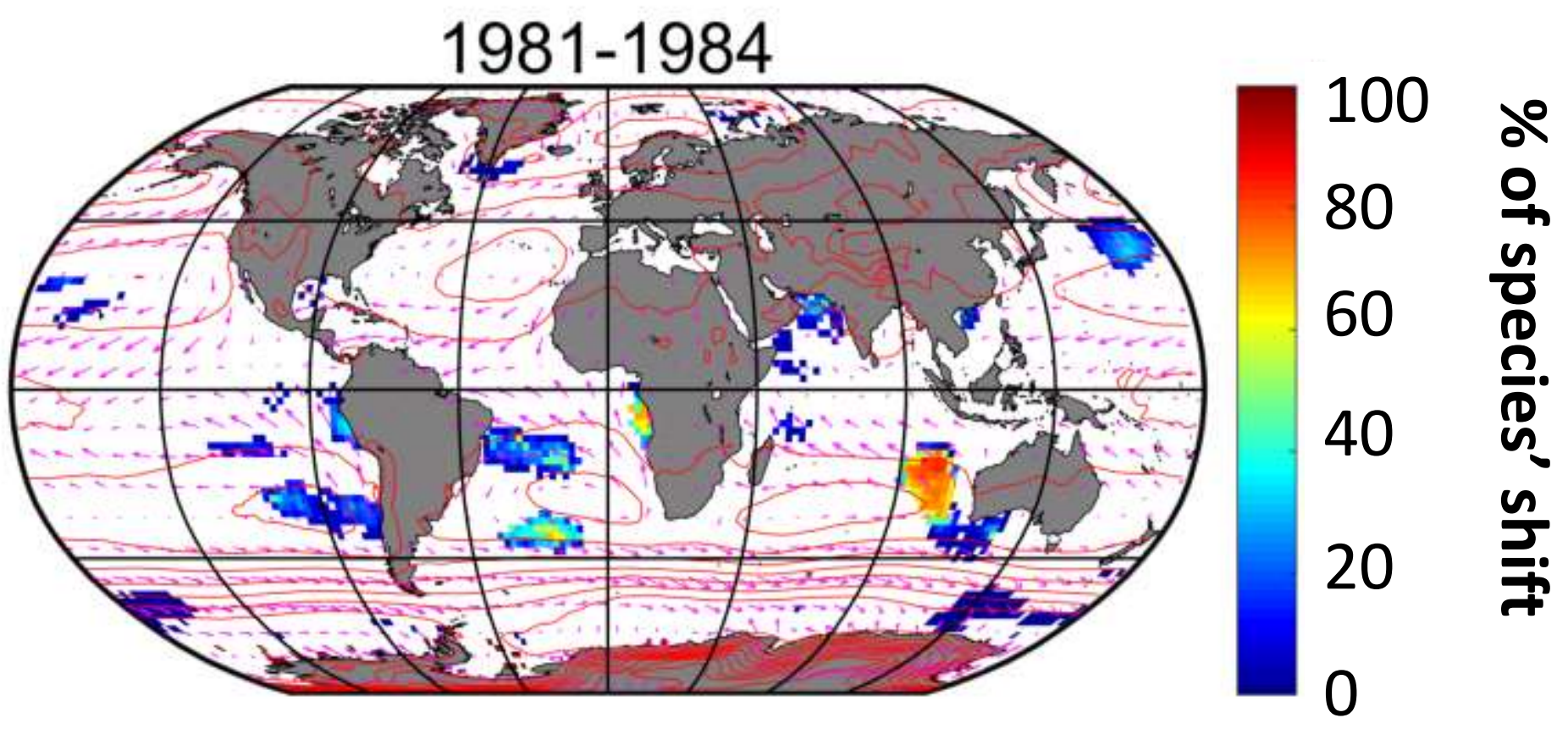

Pink arrow: direction and strengh of mean annual wind (1960-2015) Red line: isobar based on annual SLP for the period 1960-2015 White area: no significant species' shift (threshold $>3$ ) 


\section{Percentage of species' shift into a community}

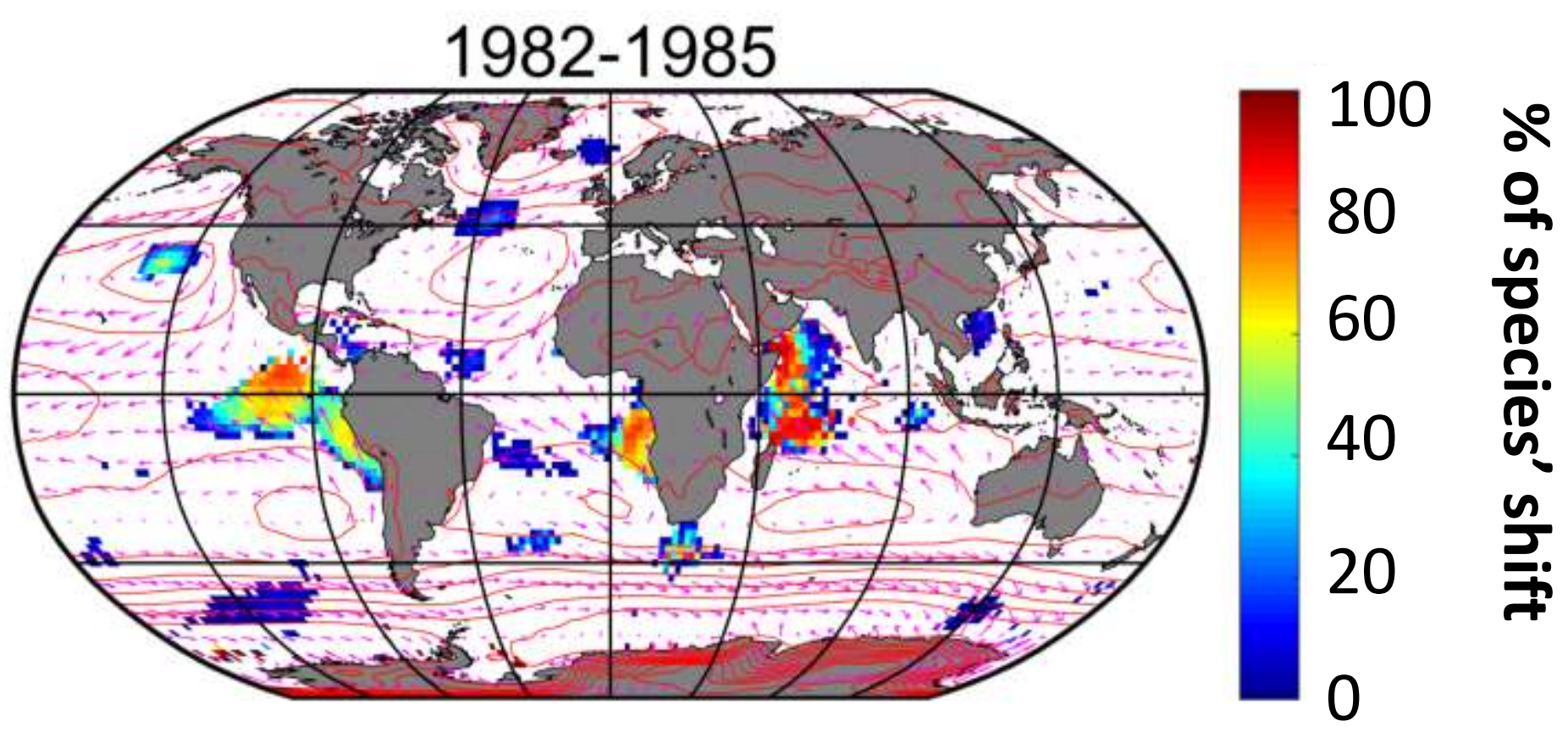

Pink arrow: direction and strengh of mean annual wind (1960-2015) Red line: isobar based on annual SLP for the period 1960-2015 White area: no significant species' shift (threshold $>3$ ) 


\section{Percentage of species' shift into a community}

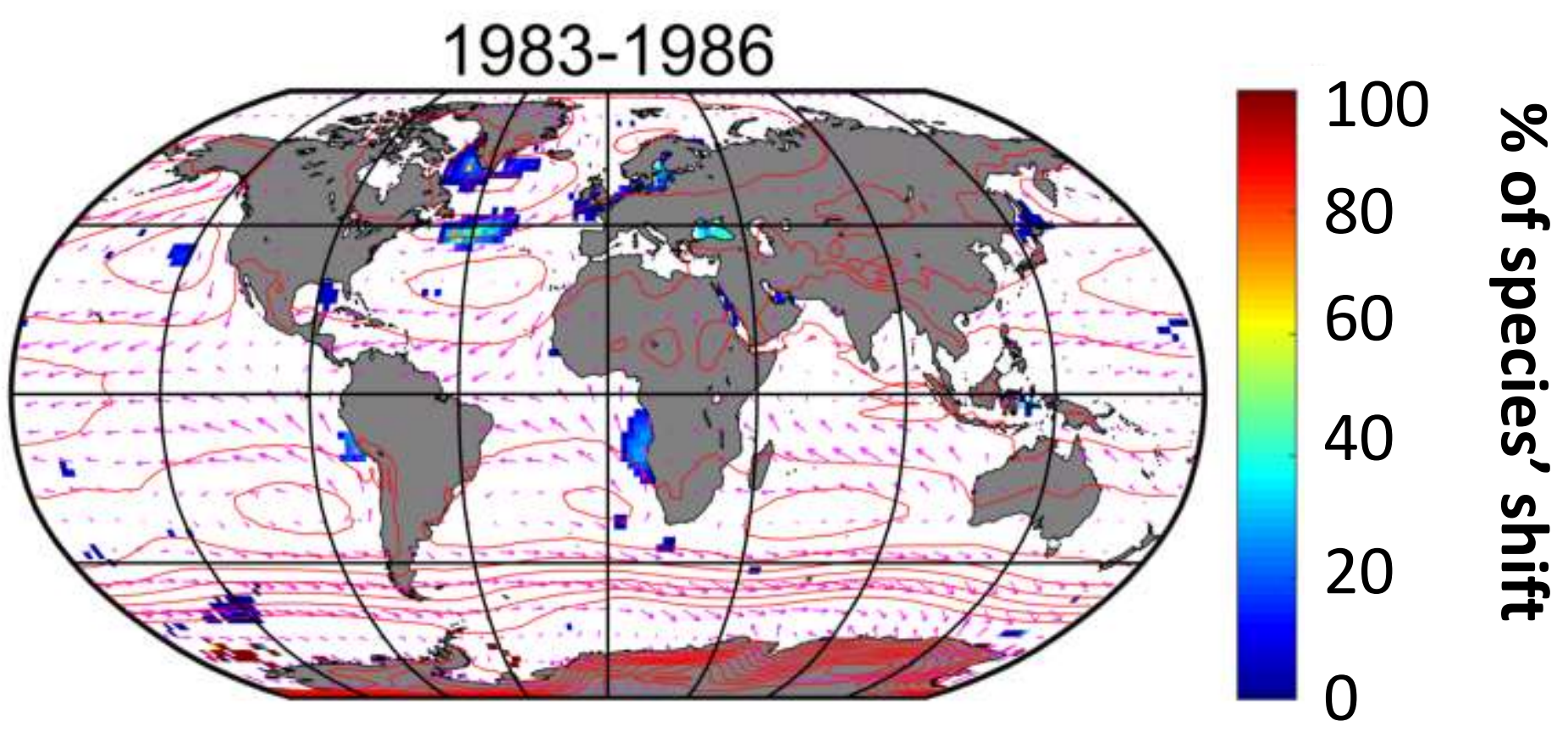

Pink arrow: direction and strengh of mean annual wind (1960-2015) Red line: isobar based on annual SLP for the period 1960-2015 White area: no significant species' shift (threshold $>3$ ) 


\section{Percentage of species' shift into a community}

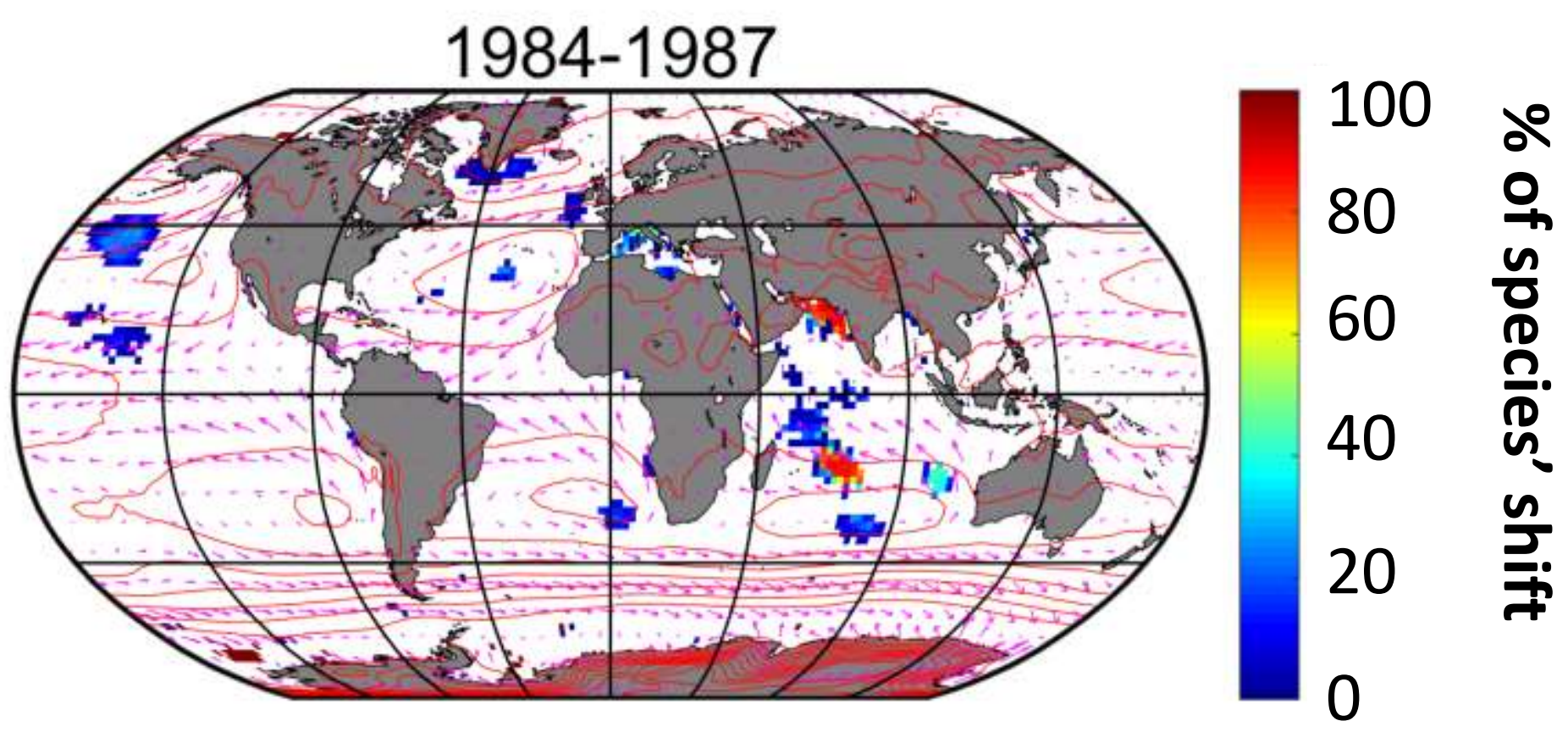

Pink arrow: direction and strengh of mean annual wind (1960-2015) Red line: isobar based on annual SLP for the period 1960-2015 White area: no significant species' shift (threshold $>3$ ) 


\section{Percentage of species' shift into a community}

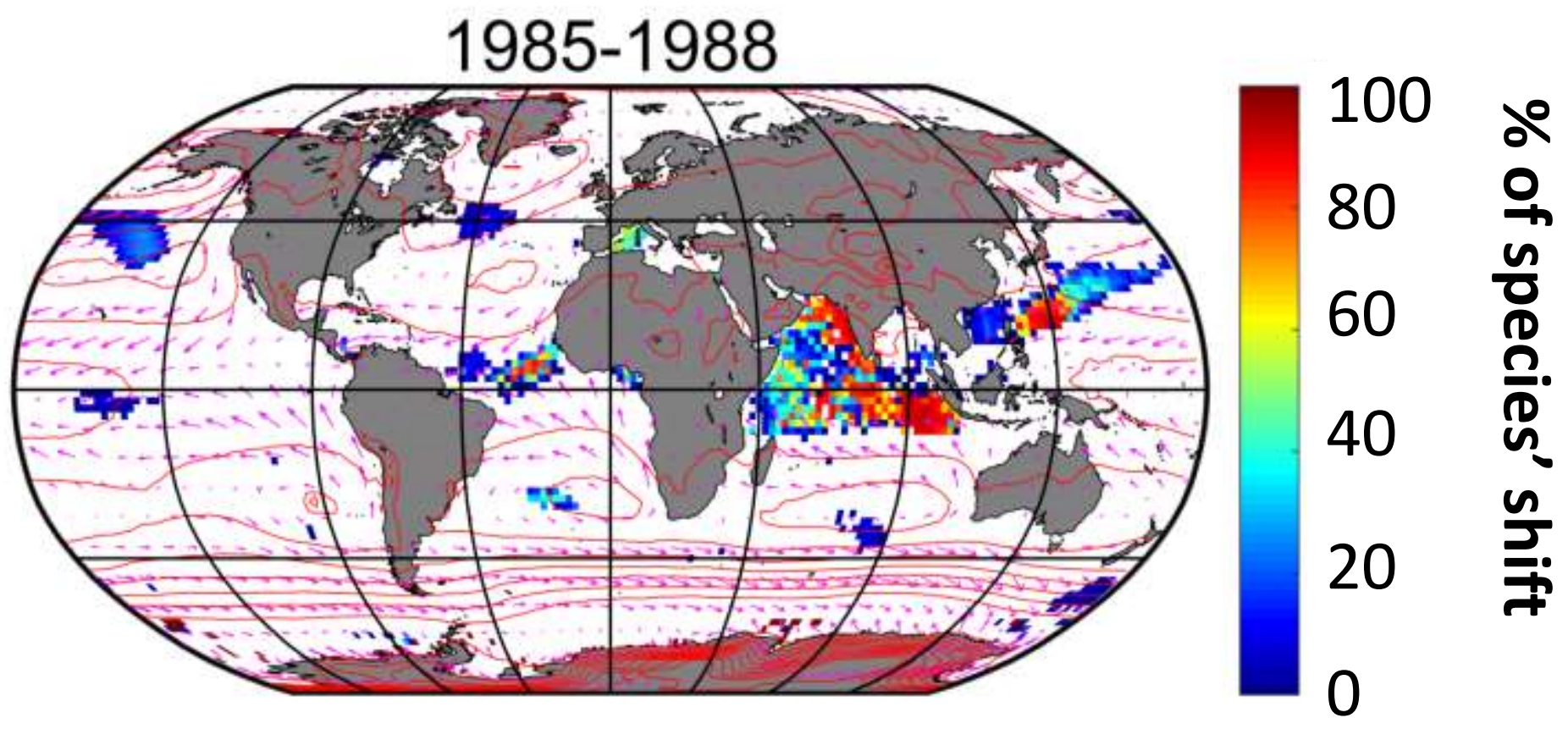

Pink arrow: direction and strengh of mean annual wind (1960-2015) Red line: isobar based on annual SLP for the period 1960-2015 White area: no significant species' shift (threshold $>3$ ) 


\section{Percentage of species' shift into a community}

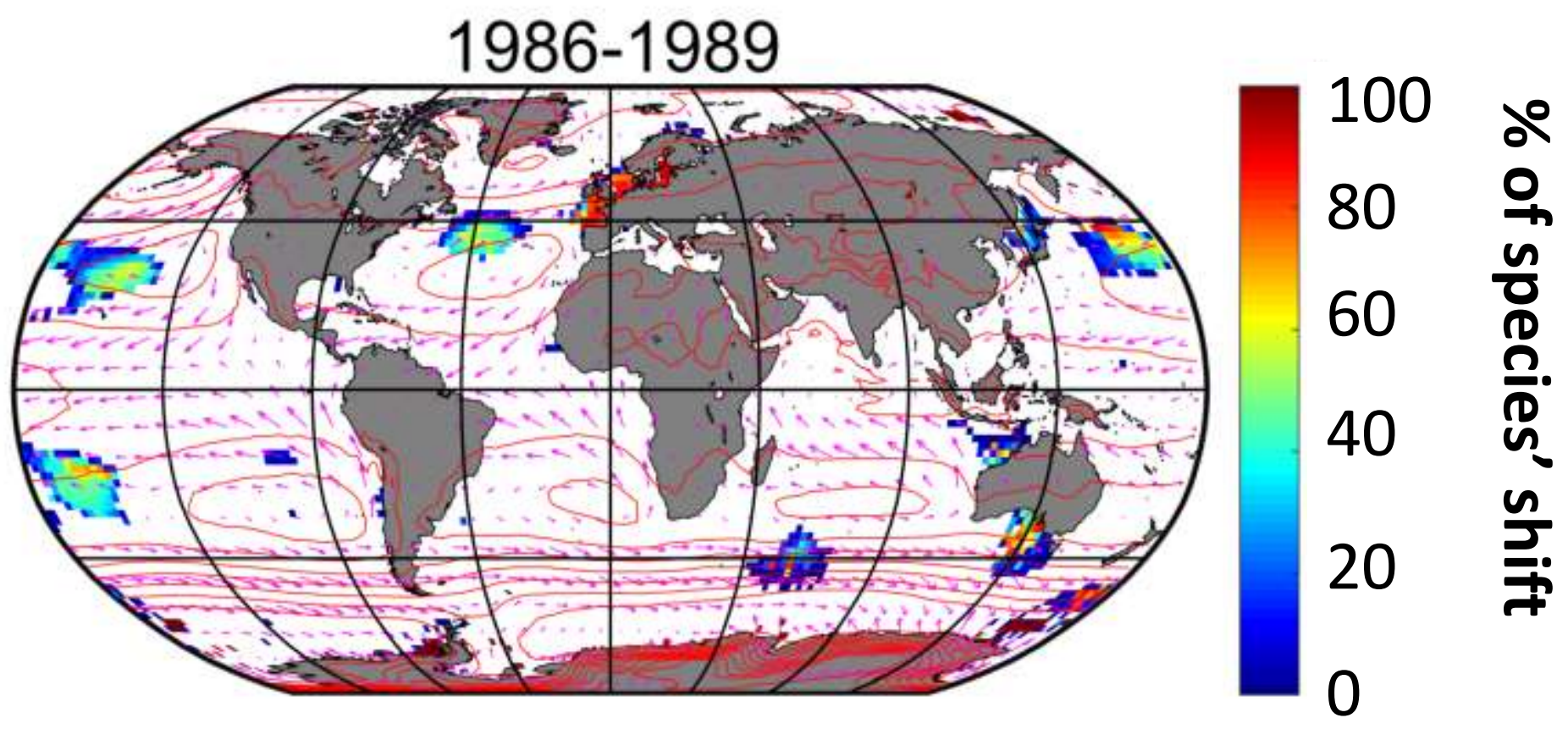

Pink arrow: direction and strengh of mean annual wind (1960-2015) Red line: isobar based on annual SLP for the period 1960-2015 White area: no significant species' shift (threshold $>3$ ) 


\section{Percentage of species' shift into a community}

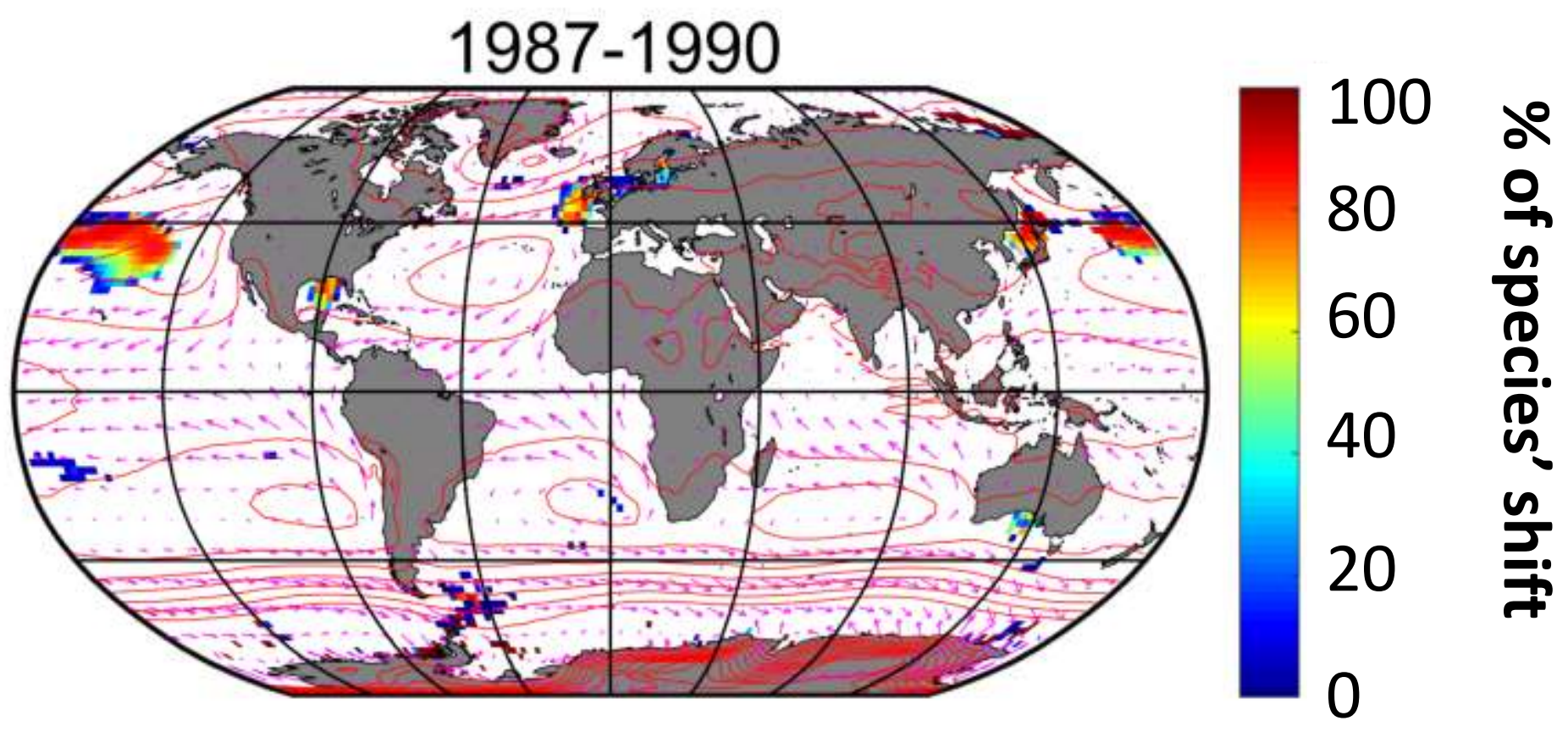

Pink arrow: direction and strengh of mean annual wind (1960-2015) Red line: isobar based on annual SLP for the period 1960-2015 White area: no significant species' shift (threshold $>3$ ) 


\section{Percentage of species' shift into a community}

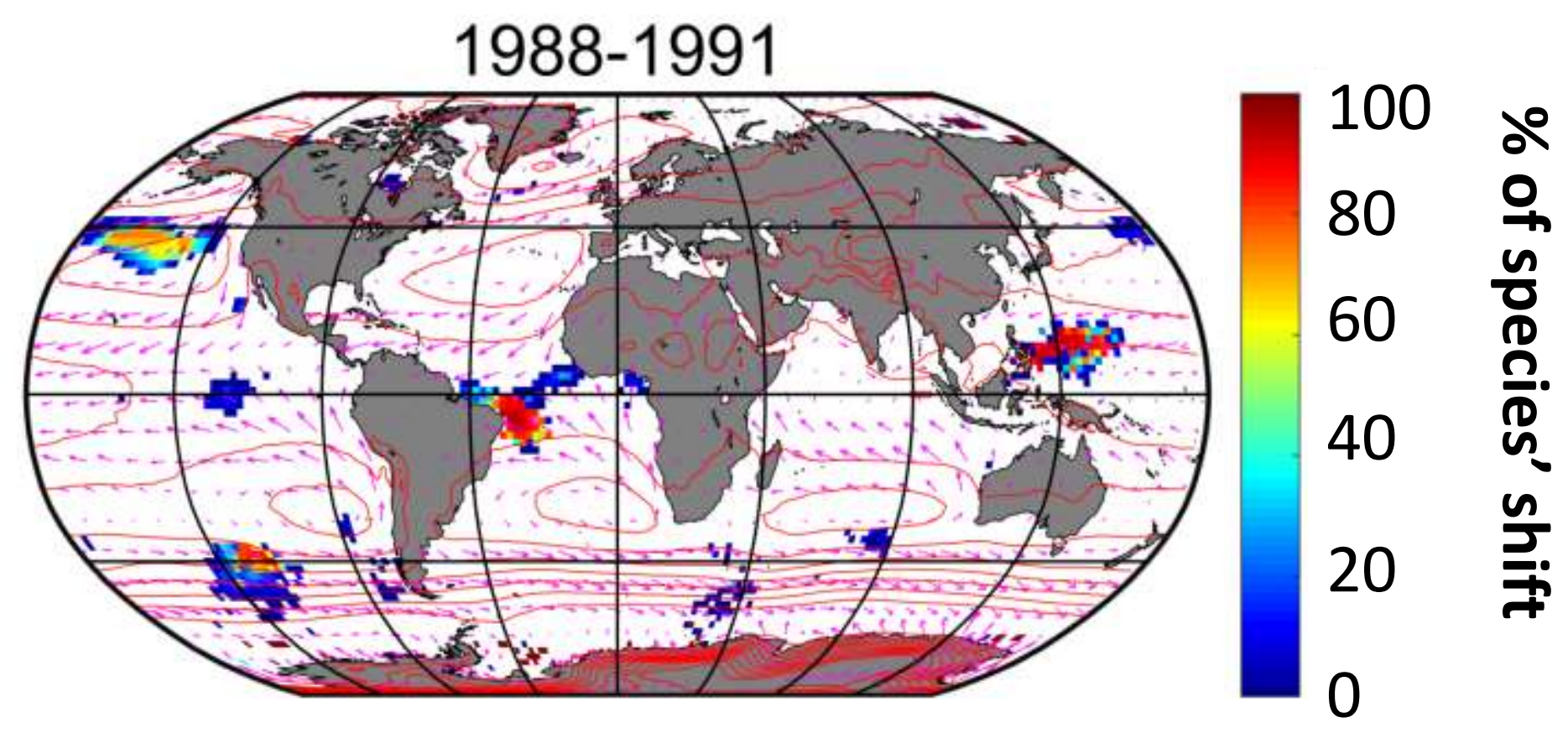

Pink arrow: direction and strengh of mean annual wind (1960-2015) Red line: isobar based on annual SLP for the period 1960-2015 White area: no significant species' shift (threshold $>3$ ) 


\section{Percentage of species' shift into a community}

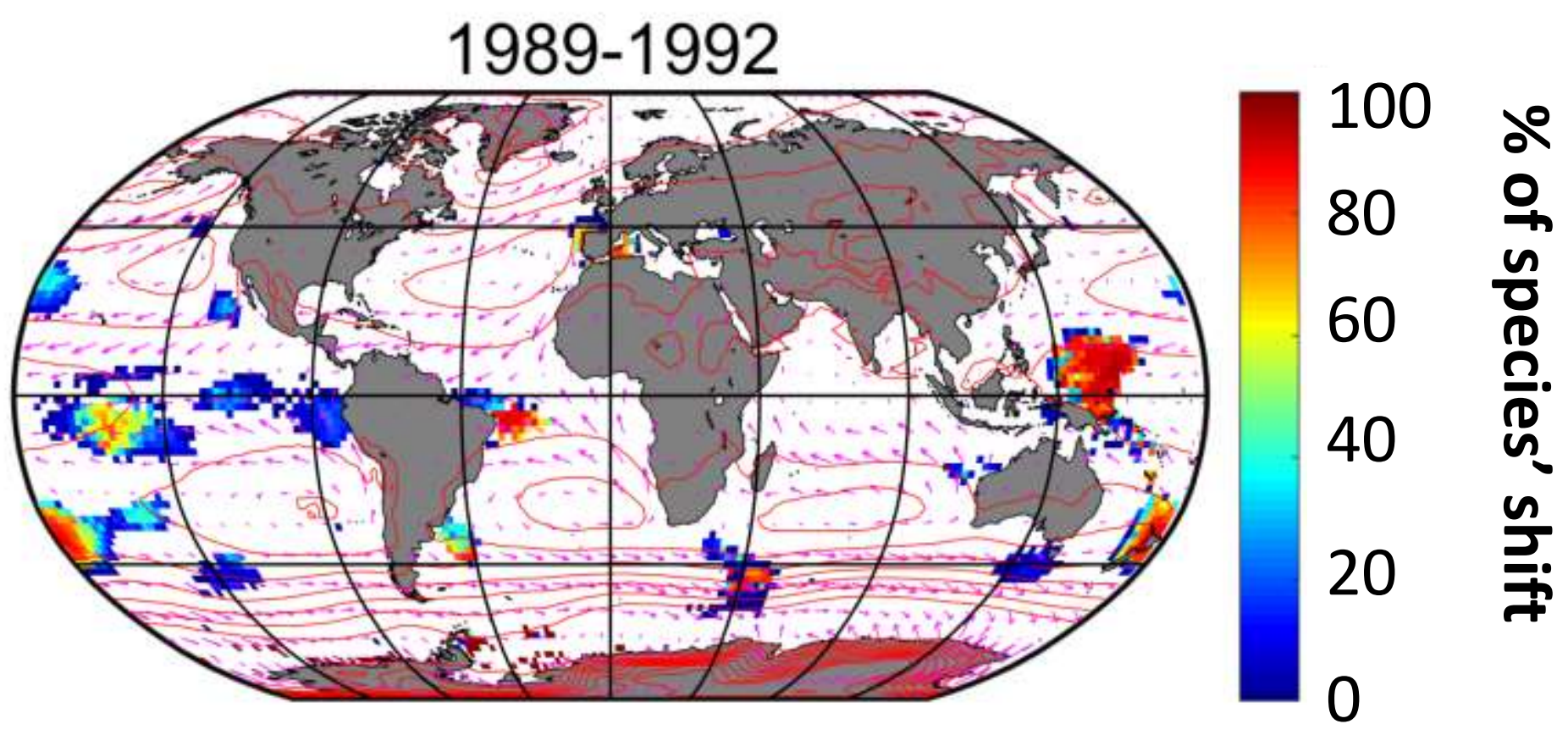

Pink arrow: direction and strengh of mean annual wind (1960-2015) Red line: isobar based on annual SLP for the period 1960-2015 White area: no significant species' shift (threshold $>3$ ) 


\section{Percentage of species' shift into a community}

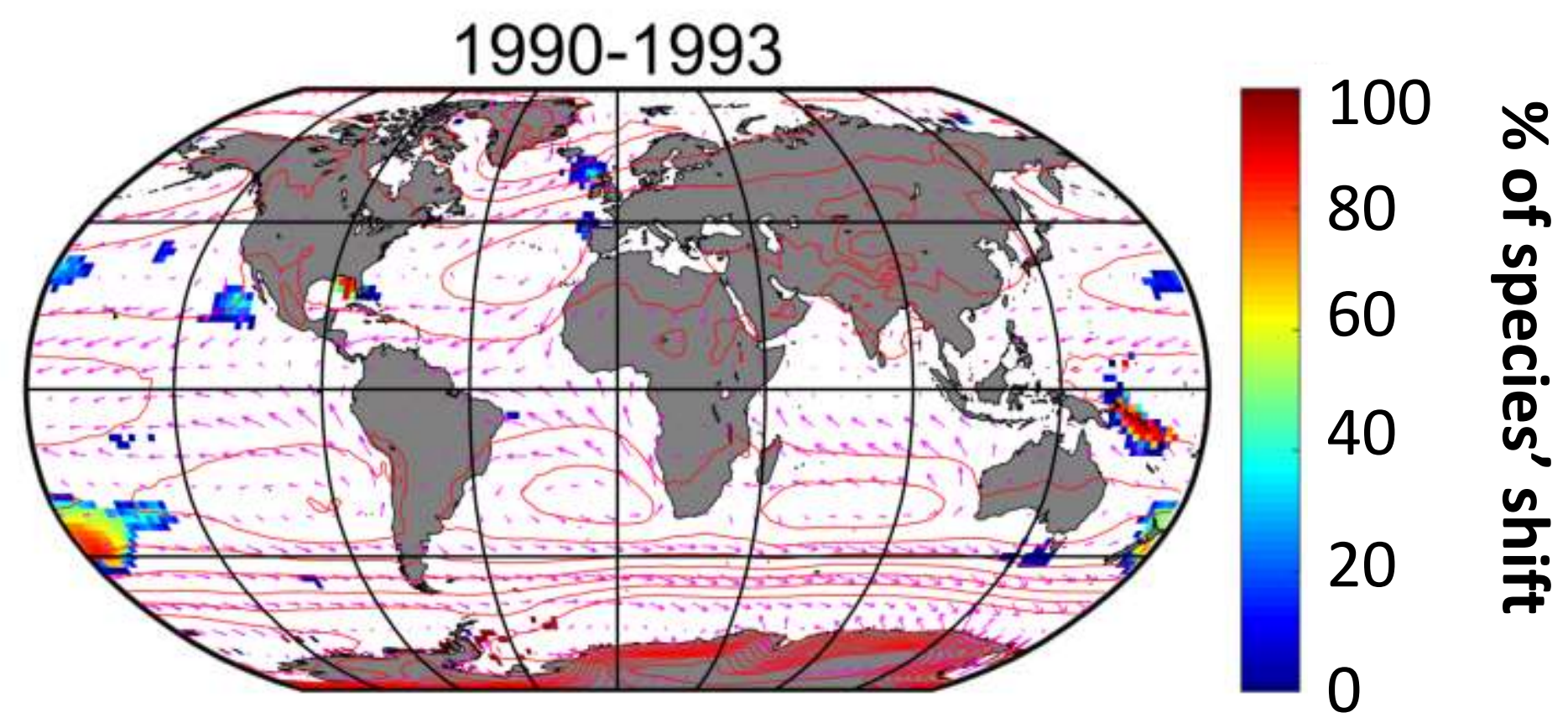

Pink arrow: direction and strengh of mean annual wind (1960-2015) Red line: isobar based on annual SLP for the period 1960-2015 White area: no significant species' shift (threshold $>3$ ) 


\section{Percentage of species' shift into a community}

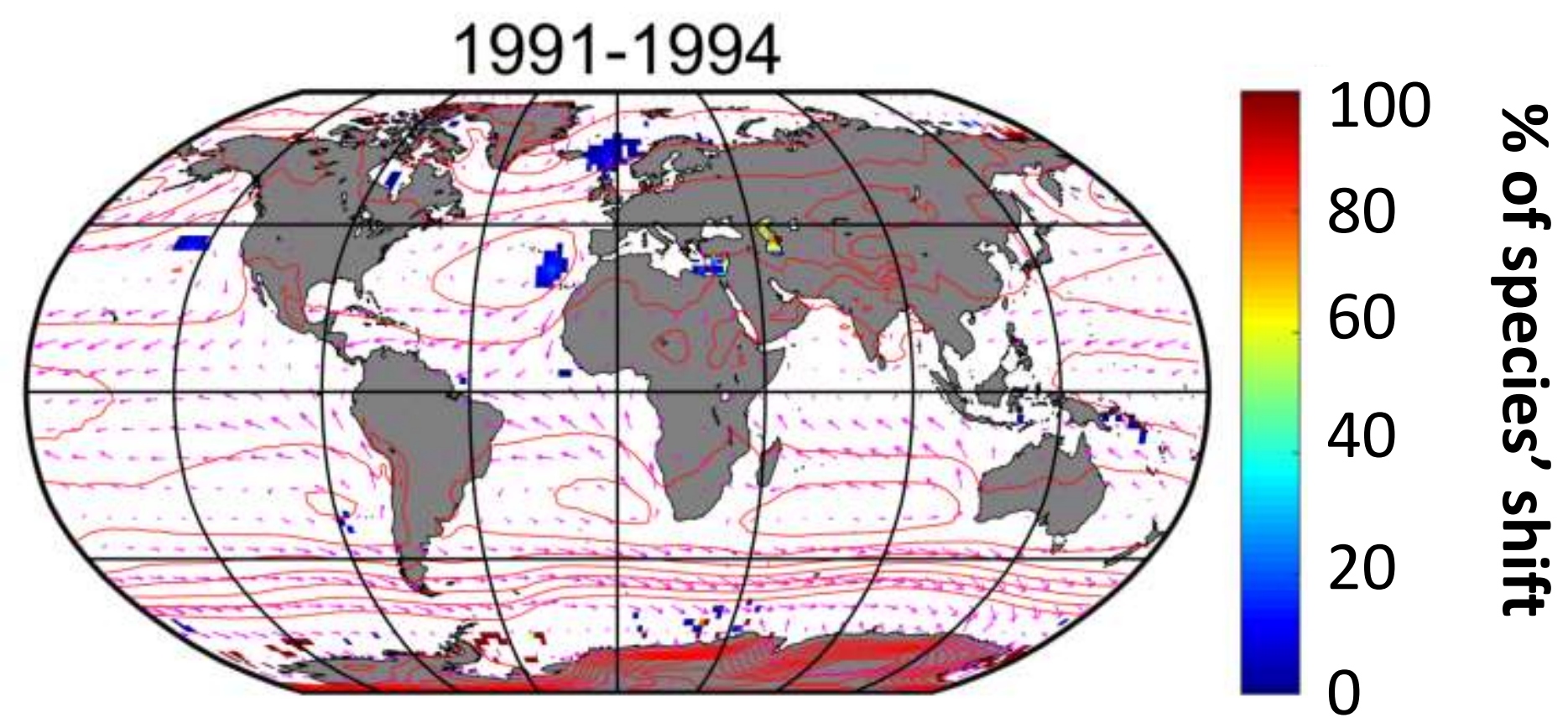

Pink arrow: direction and strengh of mean annual wind (1960-2015) Red line: isobar based on annual SLP for the period 1960-2015 White area: no significant species' shift (threshold $>3$ ) 


\section{Percentage of species' shift into a community}

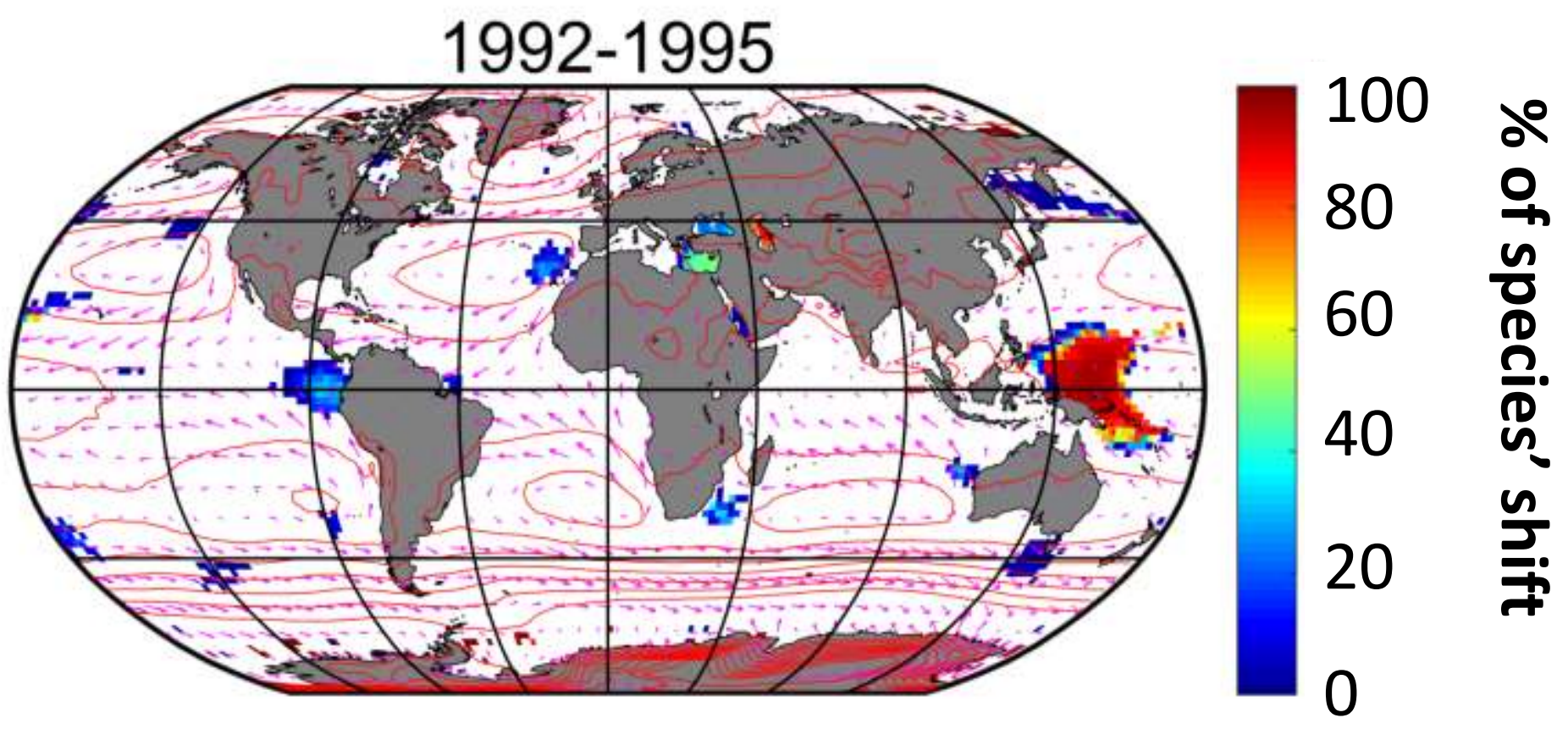

Pink arrow: direction and strengh of mean annual wind (1960-2015) Red line: isobar based on annual SLP for the period 1960-2015 White area: no significant species' shift (threshold $>3$ ) 


\section{Percentage of species' shift into a community}

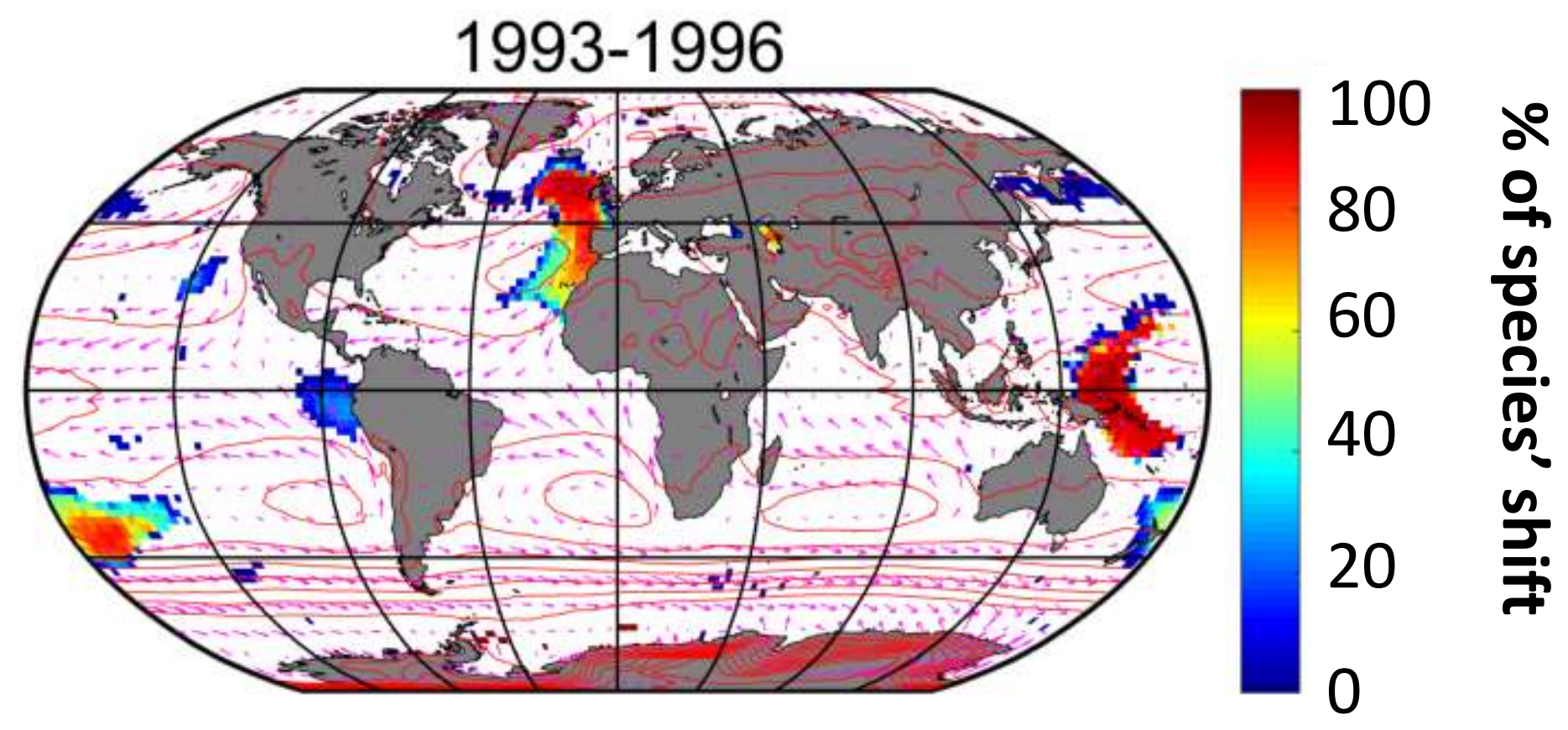

Pink arrow: direction and strengh of mean annual wind (1960-2015) Red line: isobar based on annual SLP for the period 1960-2015 White area: no significant species' shift (threshold $>3$ ) 


\section{Percentage of species' shift into a community}

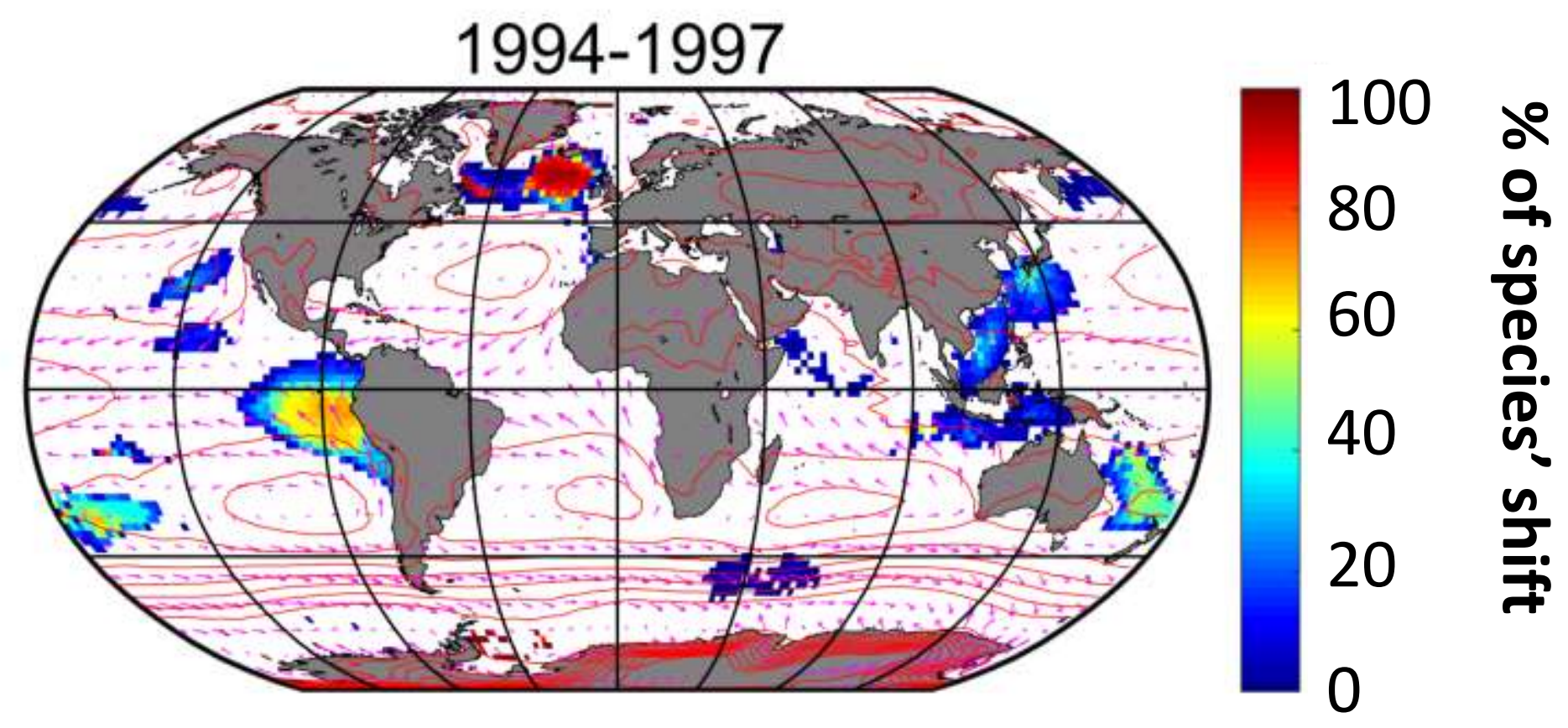

Pink arrow: direction and strengh of mean annual wind (1960-2015) Red line: isobar based on annual SLP for the period 1960-2015 White area: no significant species' shift (threshold $>3$ ) 


\section{Percentage of species' shift into a community}

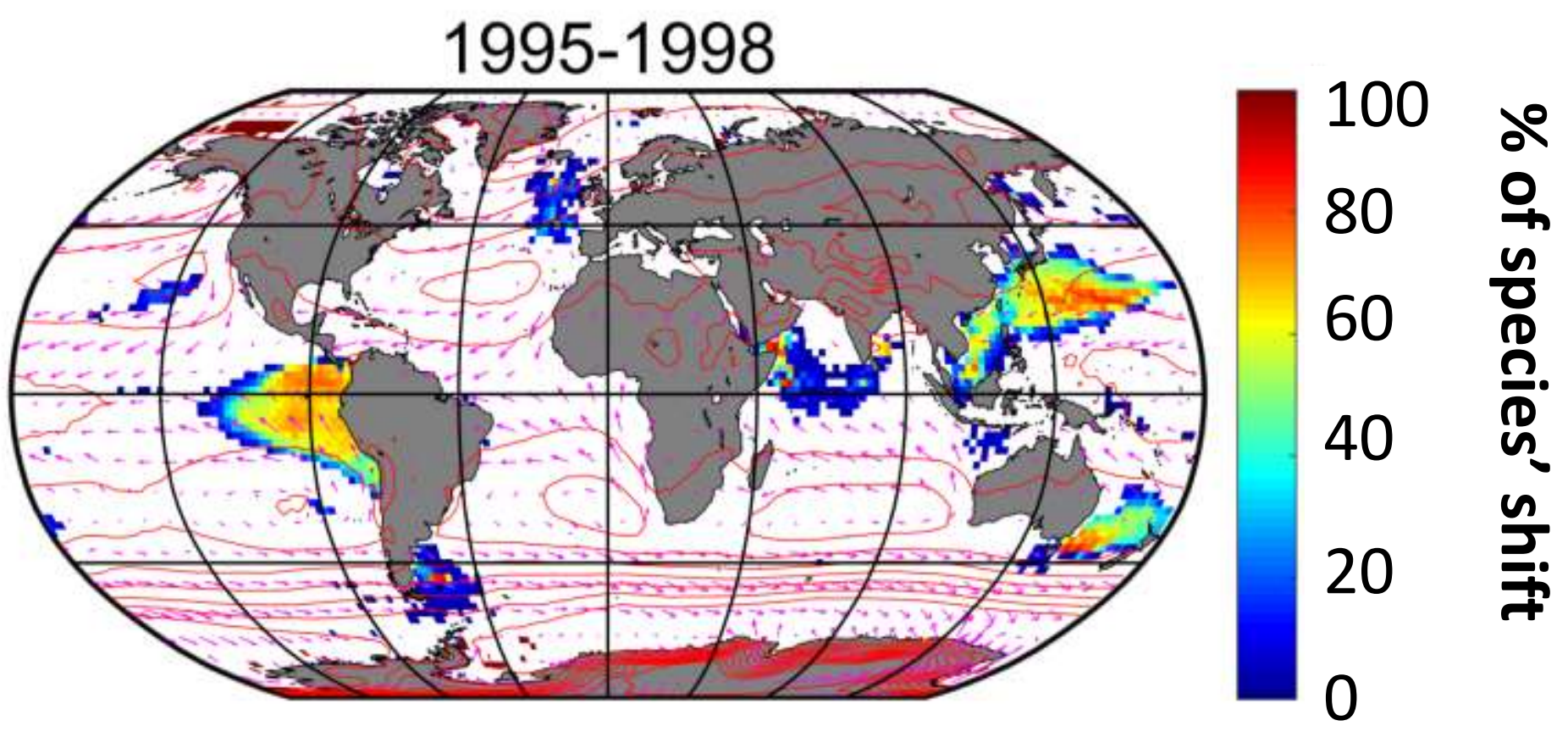

Pink arrow: direction and strengh of mean annual wind (1960-2015) Red line: isobar based on annual SLP for the period 1960-2015 White area: no significant species' shift (threshold $>3$ ) 


\section{Percentage of species' shift into a community}

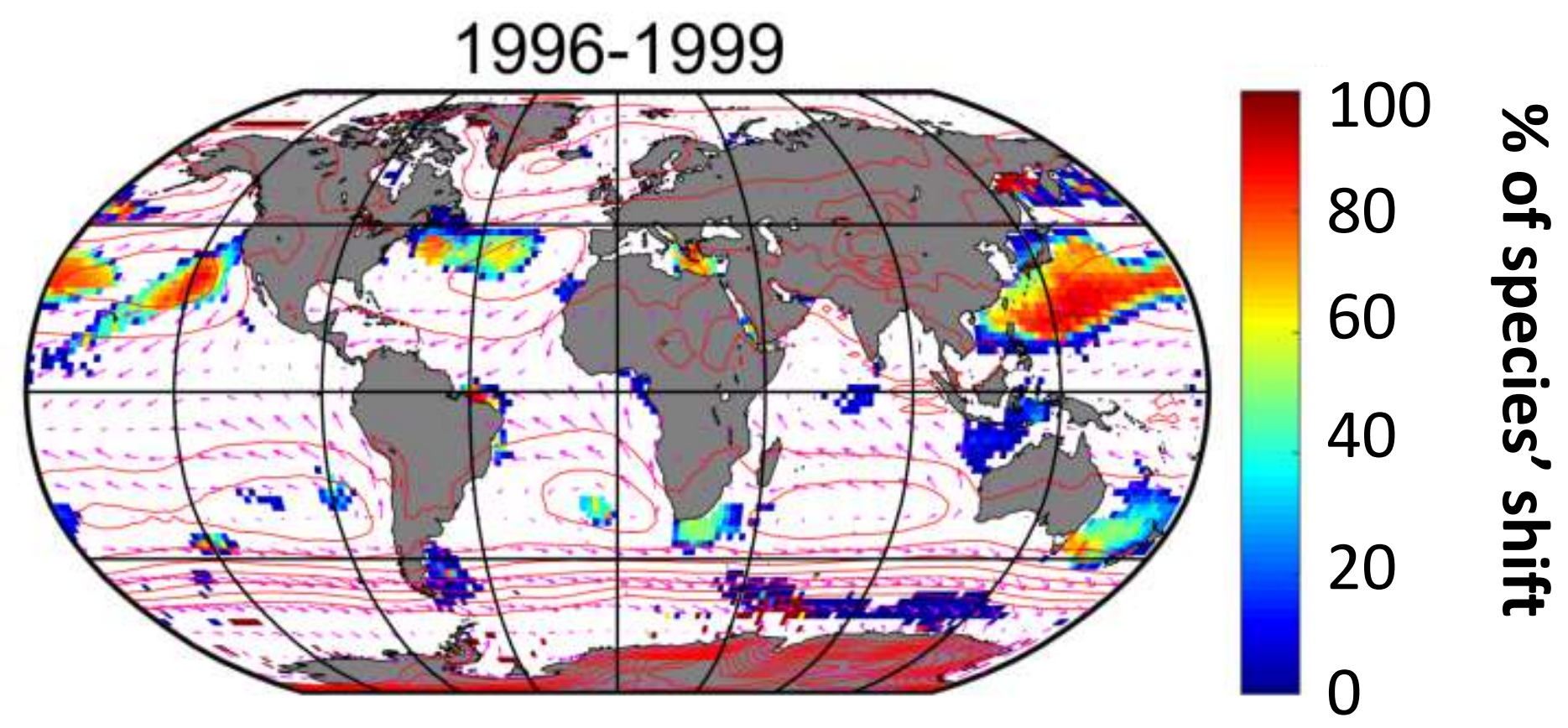

Pink arrow: direction and strengh of mean annual wind (1960-2015) Red line: isobar based on annual SLP for the period 1960-2015 White area: no significant species' shift (threshold $>3$ ) 


\section{Percentage of species' shift into a community}

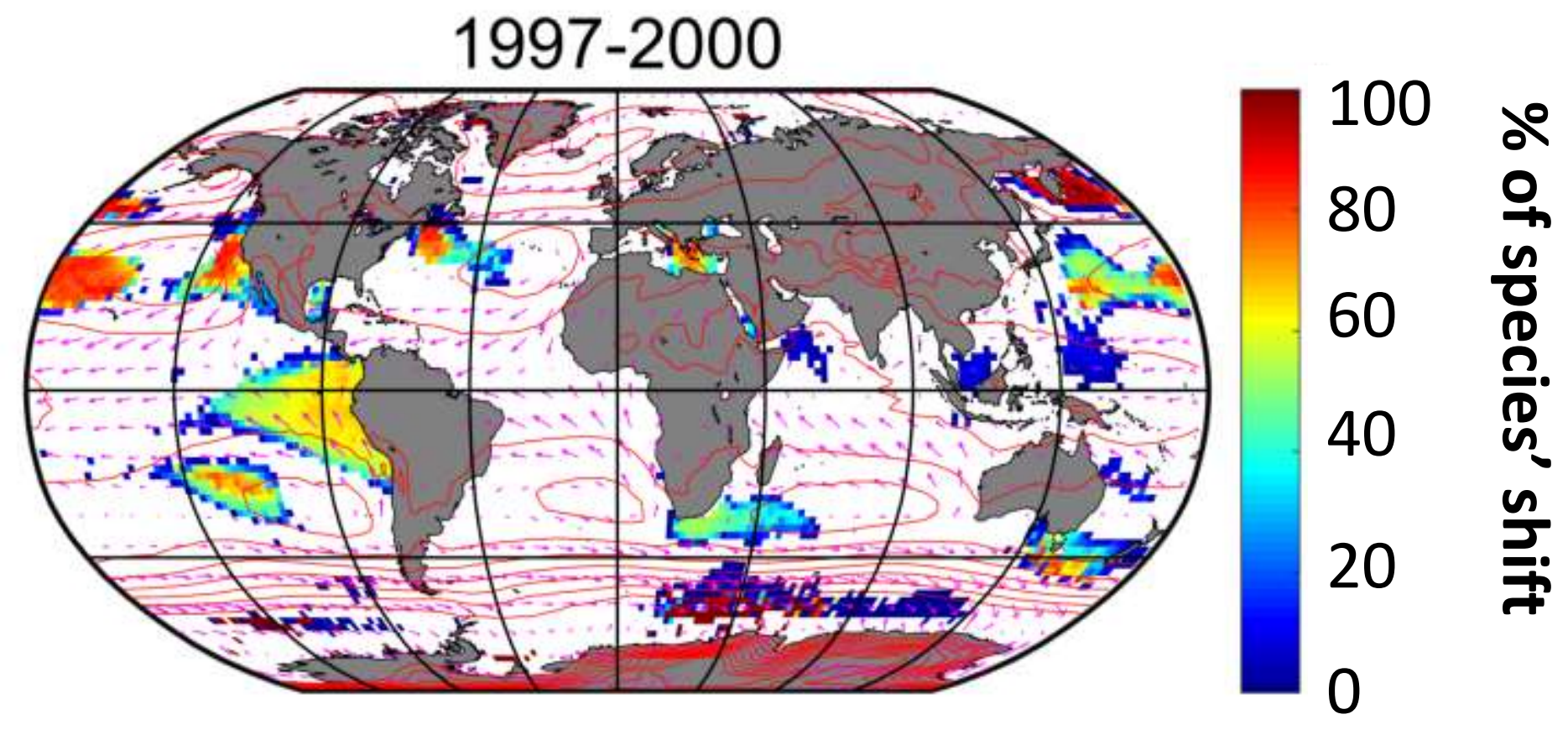

Pink arrow: direction and strengh of mean annual wind (1960-2015) Red line: isobar based on annual SLP for the period 1960-2015 White area: no significant species' shift (threshold $>3$ ) 


\section{Percentage of species' shift into a community}

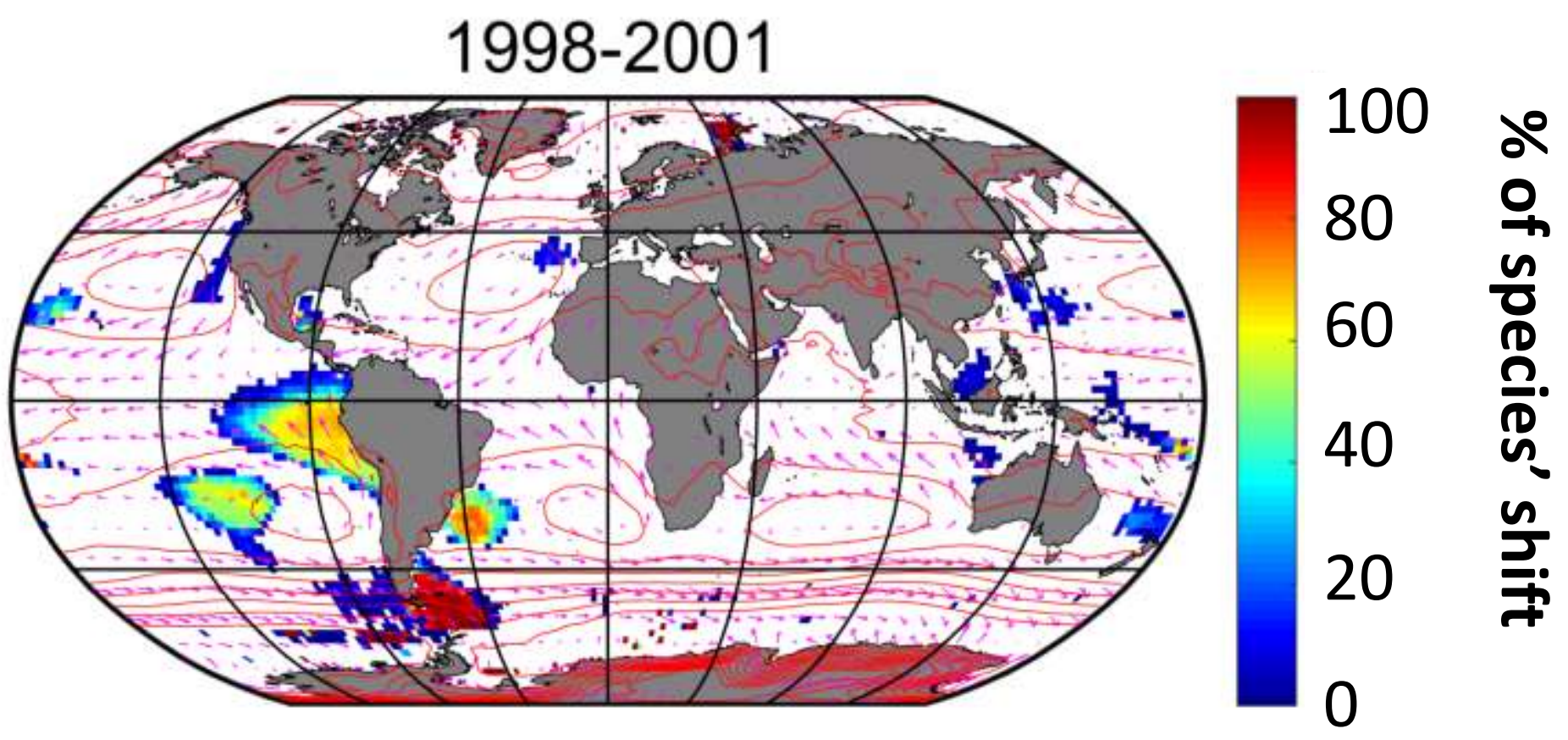

Pink arrow: direction and strengh of mean annual wind (1960-2015) Red line: isobar based on annual SLP for the period 1960-2015 White area: no significant species' shift (threshold $>3$ ) 


\section{Percentage of species' shift into a community}

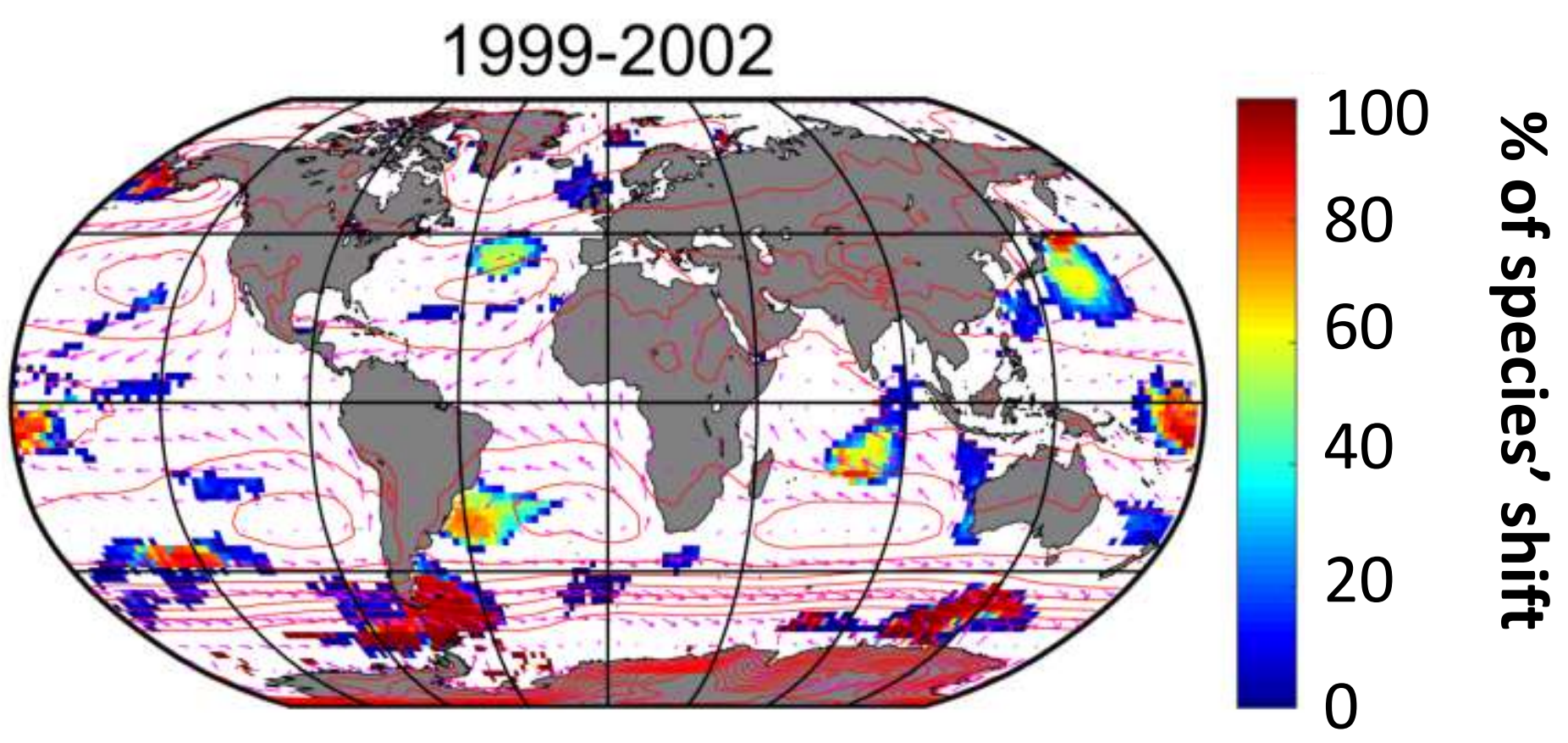

Pink arrow: direction and strengh of mean annual wind (1960-2015) Red line: isobar based on annual SLP for the period 1960-2015 White area: no significant species' shift (threshold $>3$ ) 


\section{Percentage of species' shift into a community}

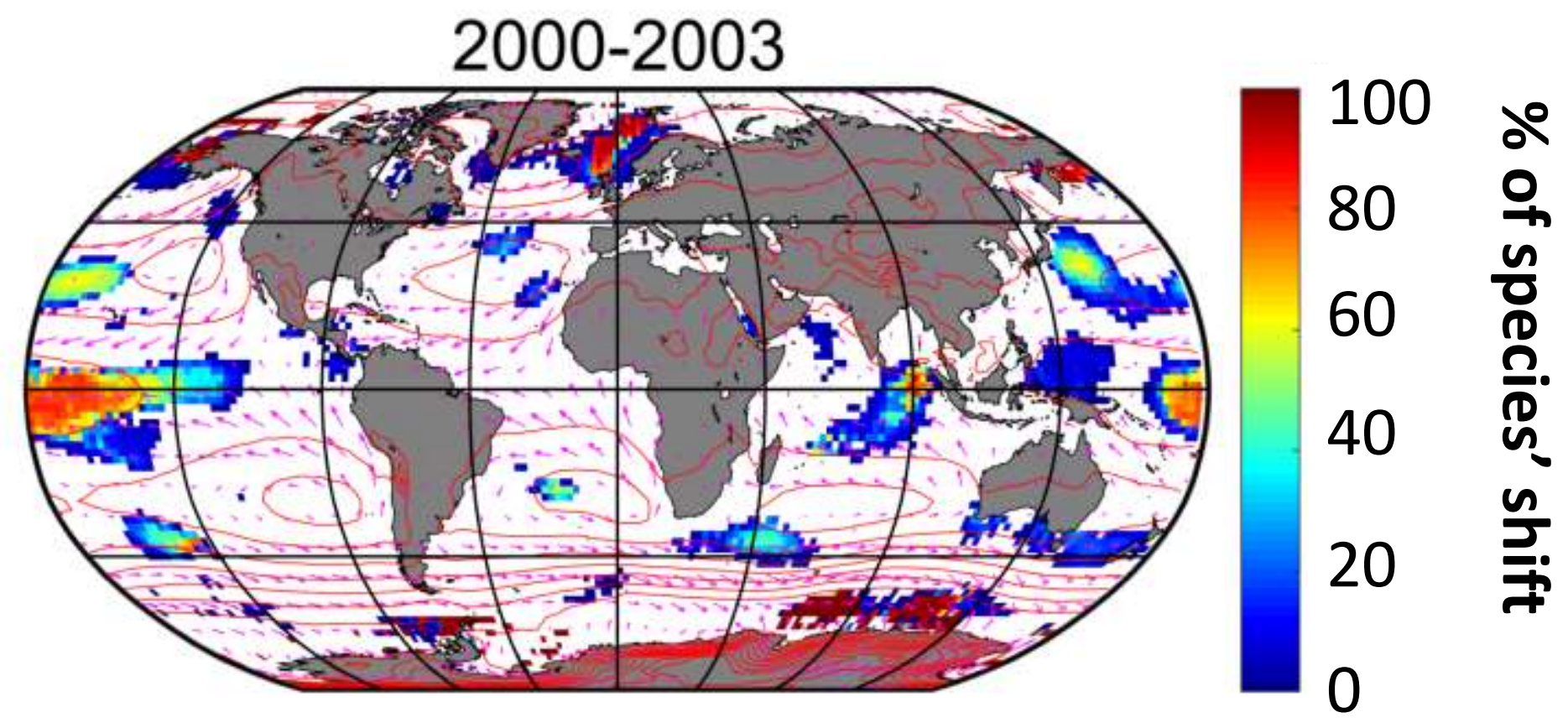

Pink arrow: direction and strengh of mean annual wind (1960-2015) Red line: isobar based on annual SLP for the period 1960-2015 White area: no significant species' shift (threshold $>3$ ) 


\section{Percentage of species' shift into a community}

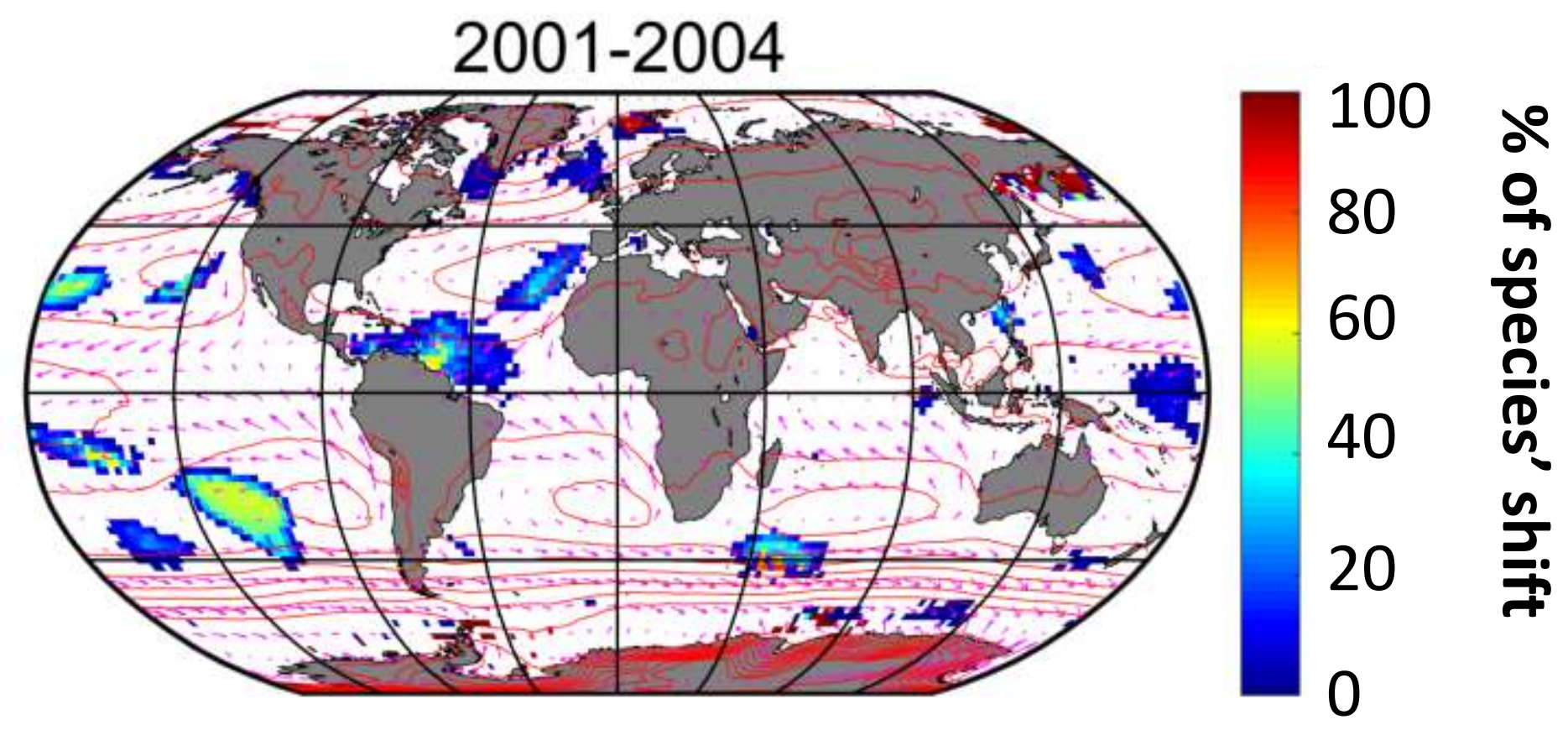

Pink arrow: direction and strengh of mean annual wind (1960-2015) Red line: isobar based on annual SLP for the period 1960-2015 White area: no significant species' shift (threshold $>3$ ) 


\section{Percentage of species' shift into a community}

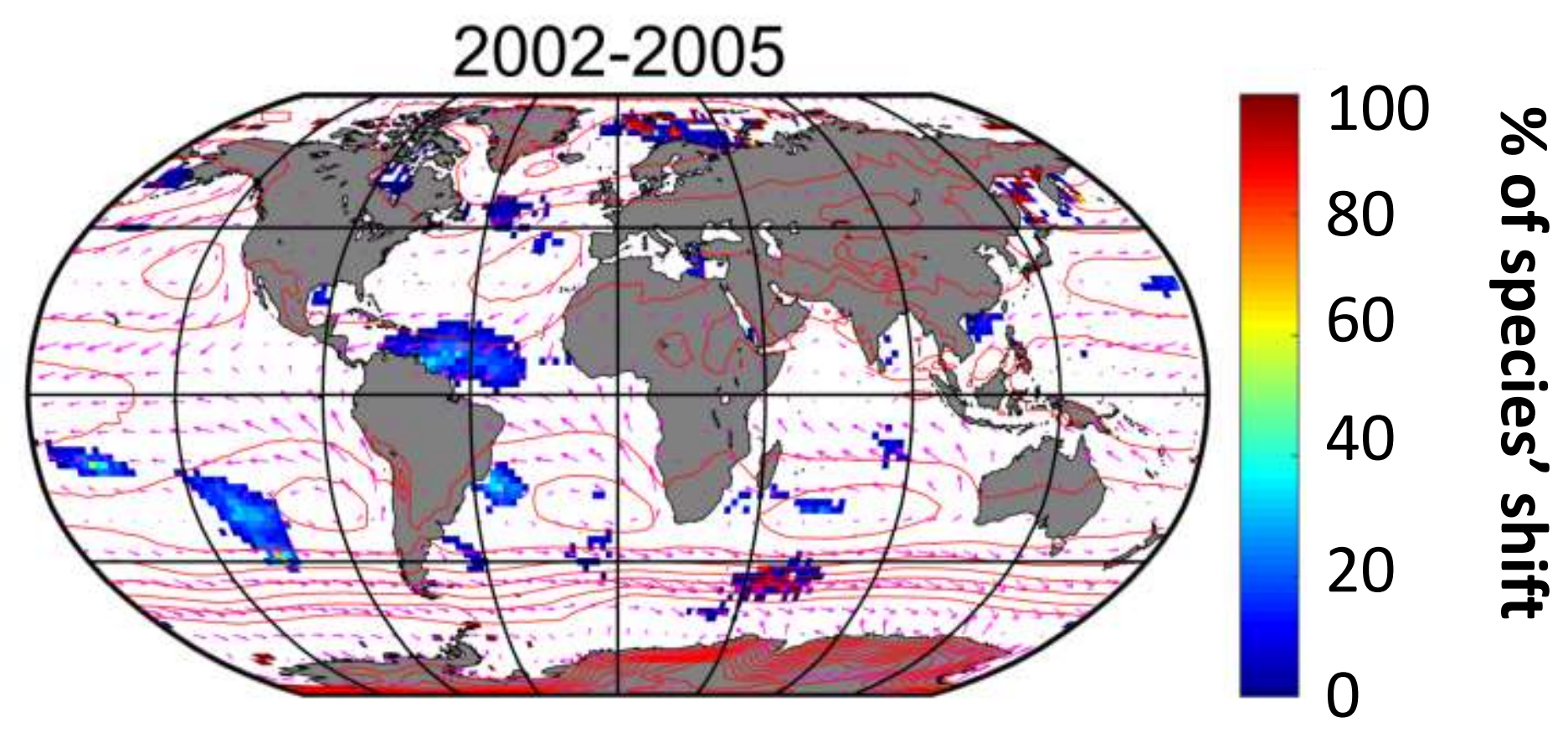

Pink arrow: direction and strengh of mean annual wind (1960-2015) Red line: isobar based on annual SLP for the period 1960-2015 White area: no significant species' shift (threshold $>3$ ) 


\section{Percentage of species' shift into a community}

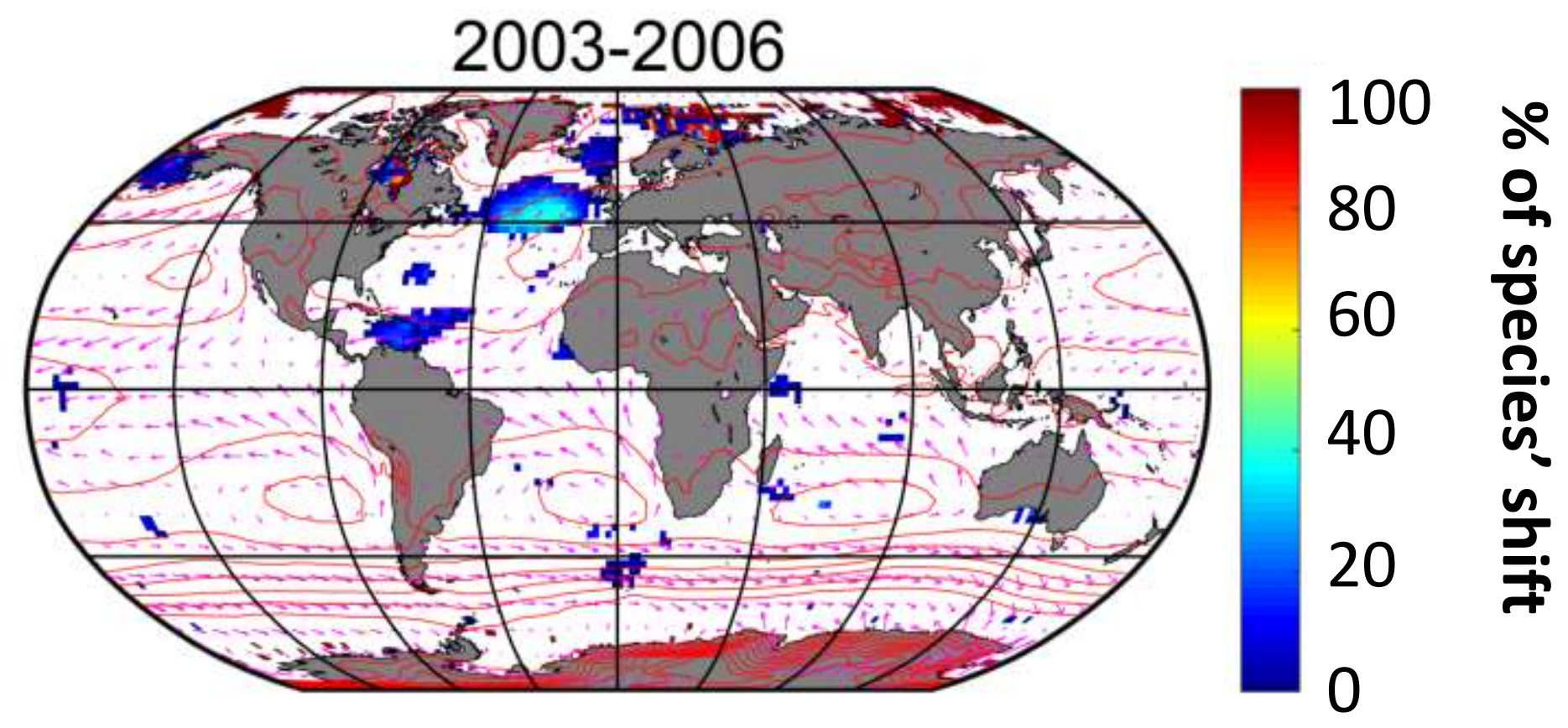

Pink arrow: direction and strengh of mean annual wind (1960-2015) Red line: isobar based on annual SLP for the period 1960-2015 White area: no significant species' shift (threshold $>3$ ) 


\section{Percentage of species' shift into a community}

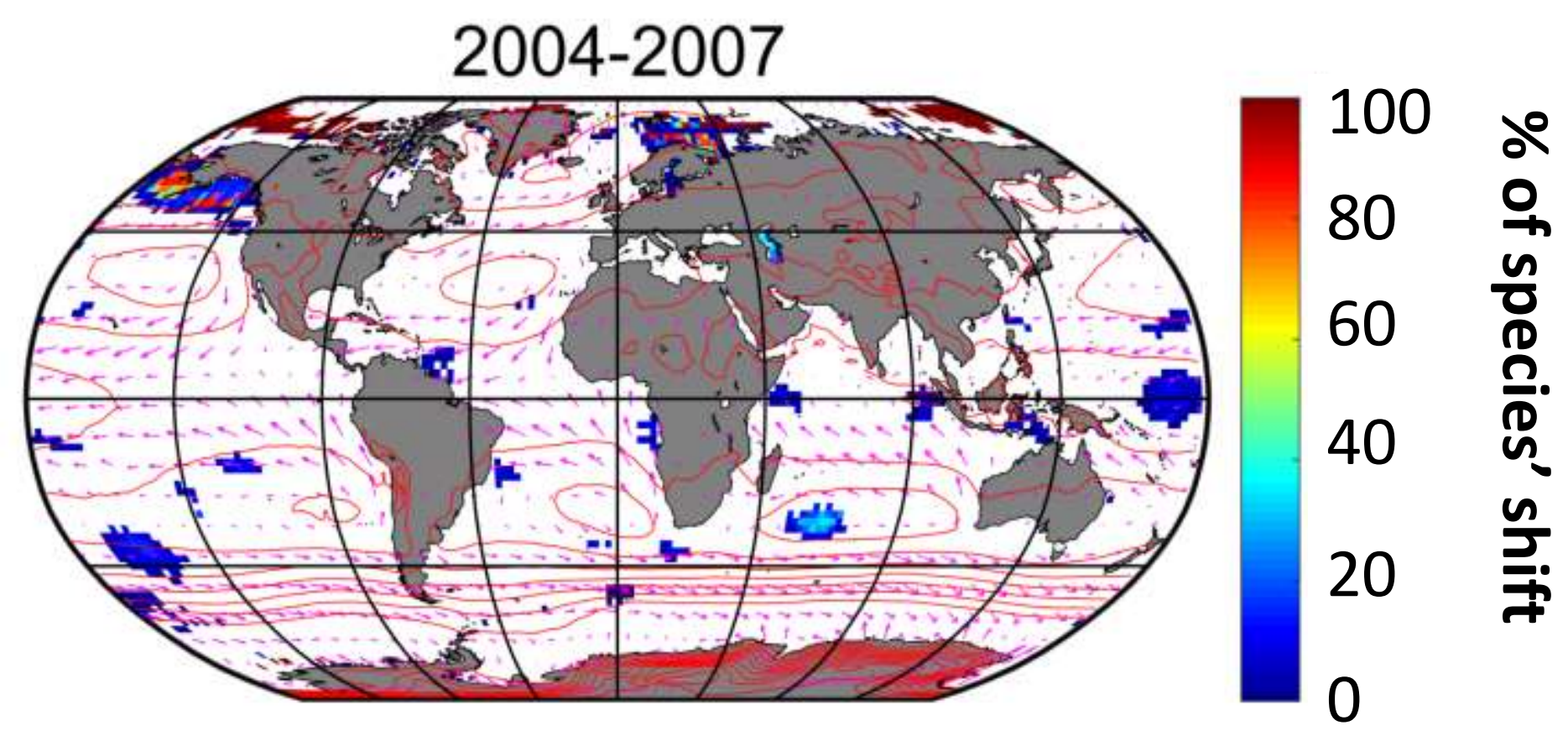

Pink arrow: direction and strengh of mean annual wind (1960-2015) Red line: isobar based on annual SLP for the period 1960-2015 White area: no significant species' shift (threshold $>3$ ) 


\section{Percentage of species' shift into a community}

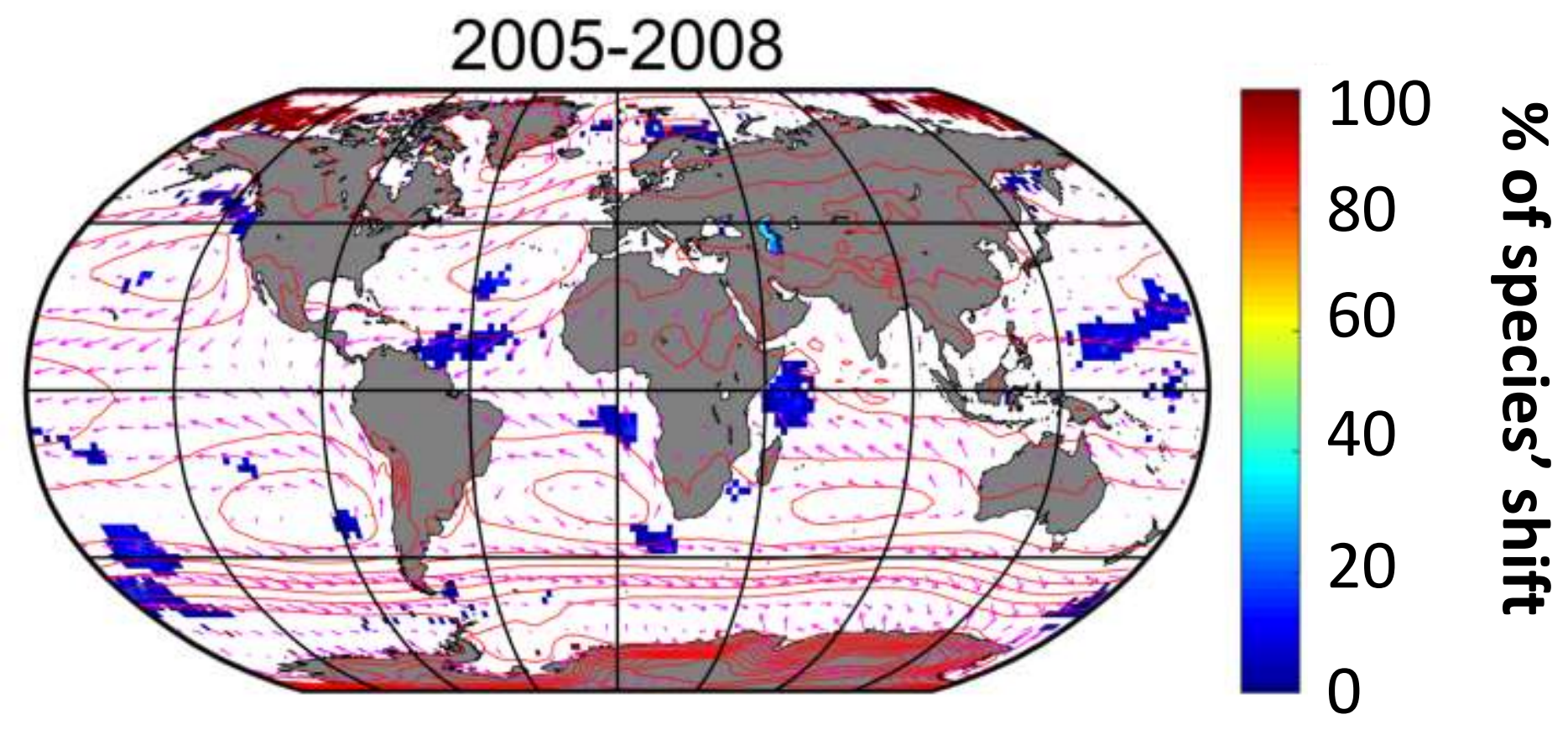

Pink arrow: direction and strengh of mean annual wind (1960-2015) Red line: isobar based on annual SLP for the period 1960-2015 White area: no significant species' shift (threshold $>3$ ) 


\section{Percentage of species' shift into a community}

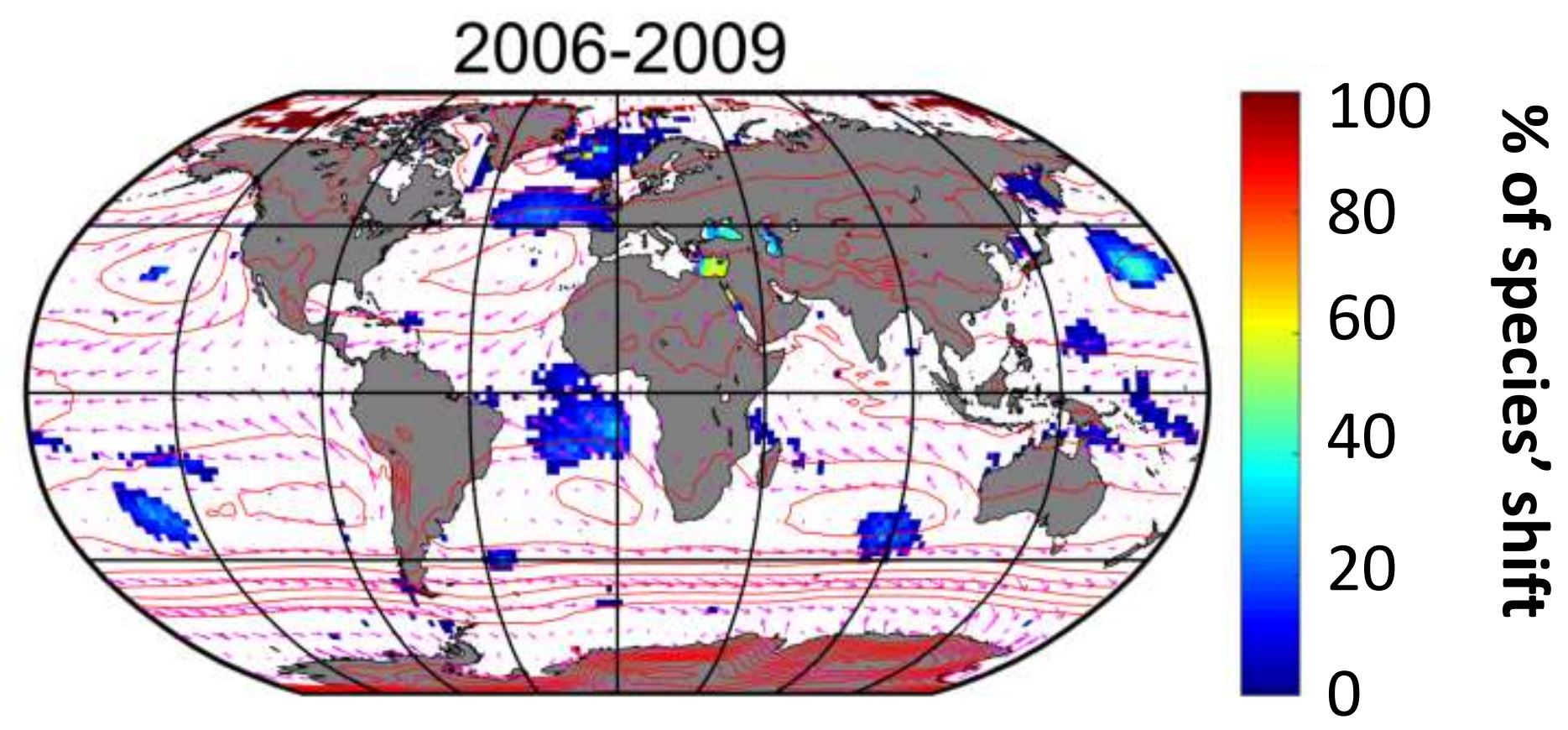

Pink arrow: direction and strengh of mean annual wind (1960-2015) Red line: isobar based on annual SLP for the period 1960-2015 White area: no significant species' shift (threshold $>3$ ) 


\section{Percentage of species' shift into a community}

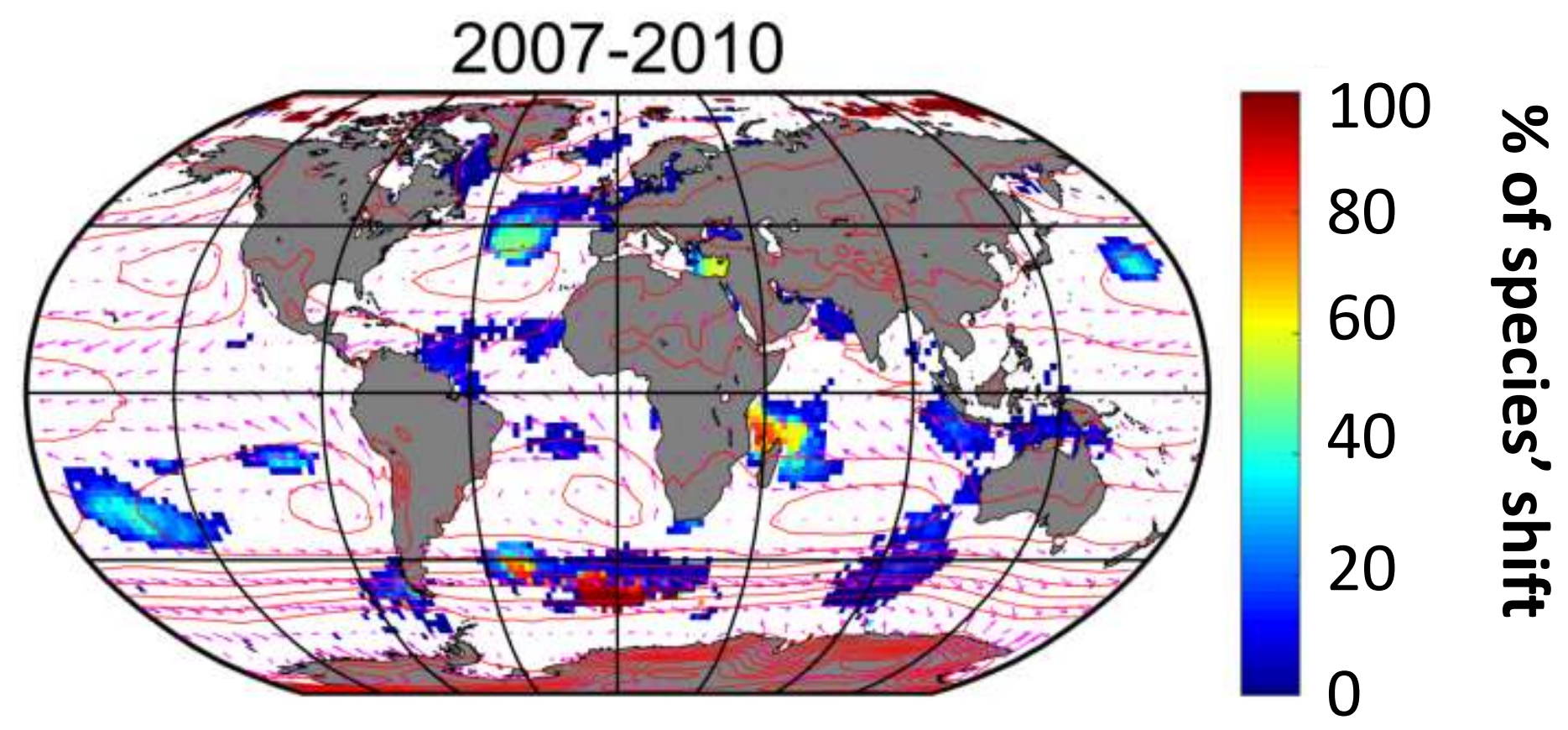

Pink arrow: direction and strengh of mean annual wind (1960-2015) Red line: isobar based on annual SLP for the period 1960-2015 White area: no significant species' shift (threshold $>3$ ) 


\section{Percentage of species' shift into a community}

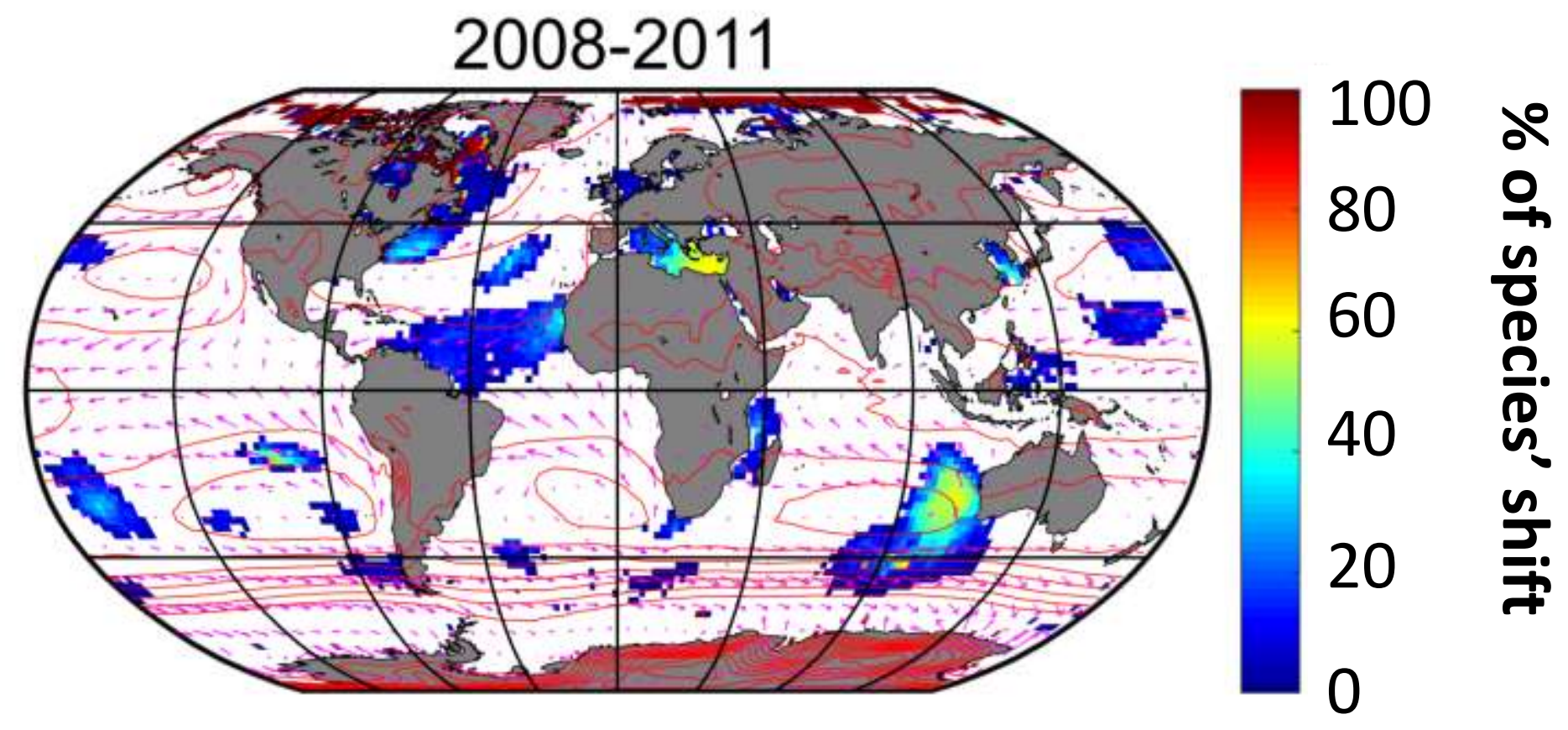

Pink arrow: direction and strengh of mean annual wind (1960-2015) Red line: isobar based on annual SLP for the period 1960-2015 White area: no significant species' shift (threshold $>3$ ) 


\section{Percentage of species' shift into a community}

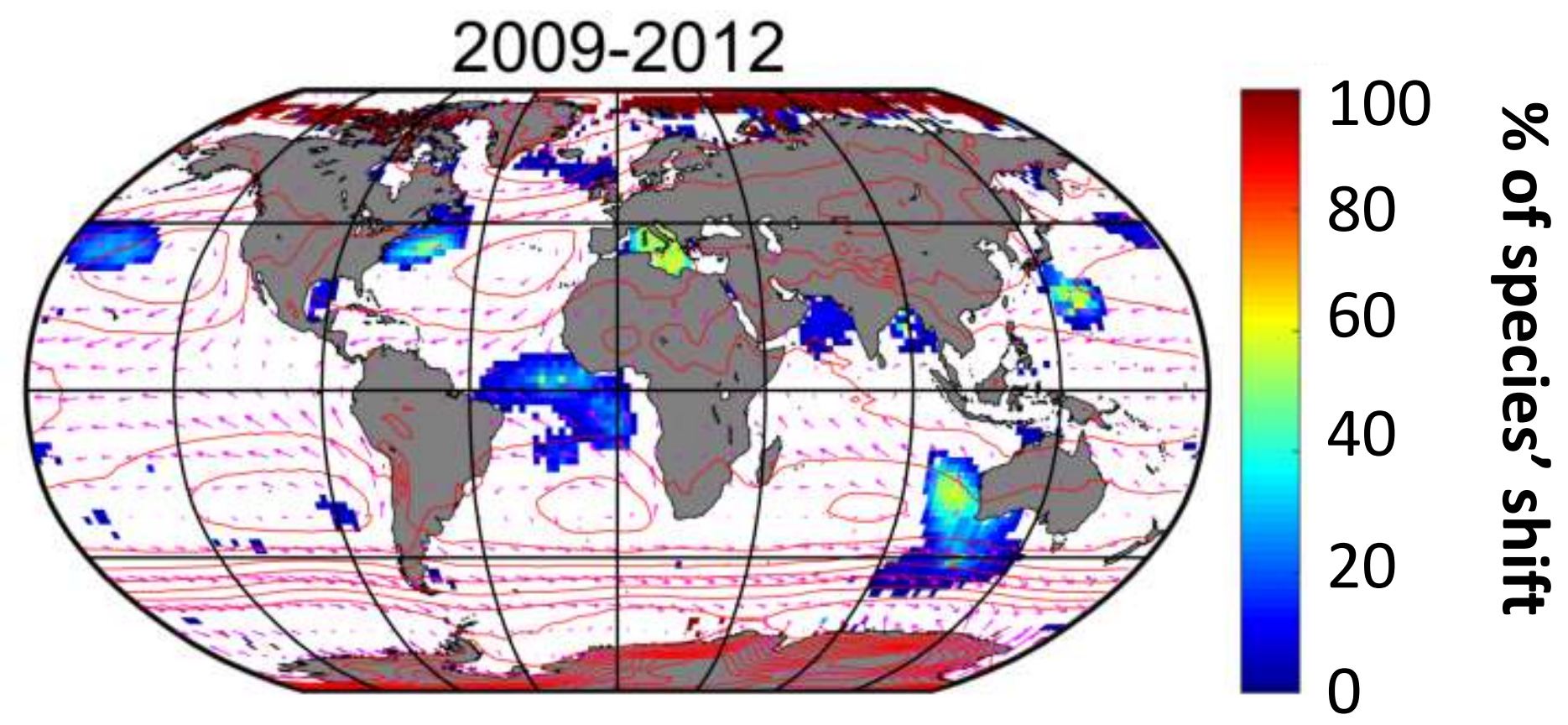

Pink arrow: direction and strengh of mean annual wind (1960-2015) Red line: isobar based on annual SLP for the period 1960-2015 White area: no significant species' shift (threshold $>3$ ) 


\section{Percentage of species' shift into a community}

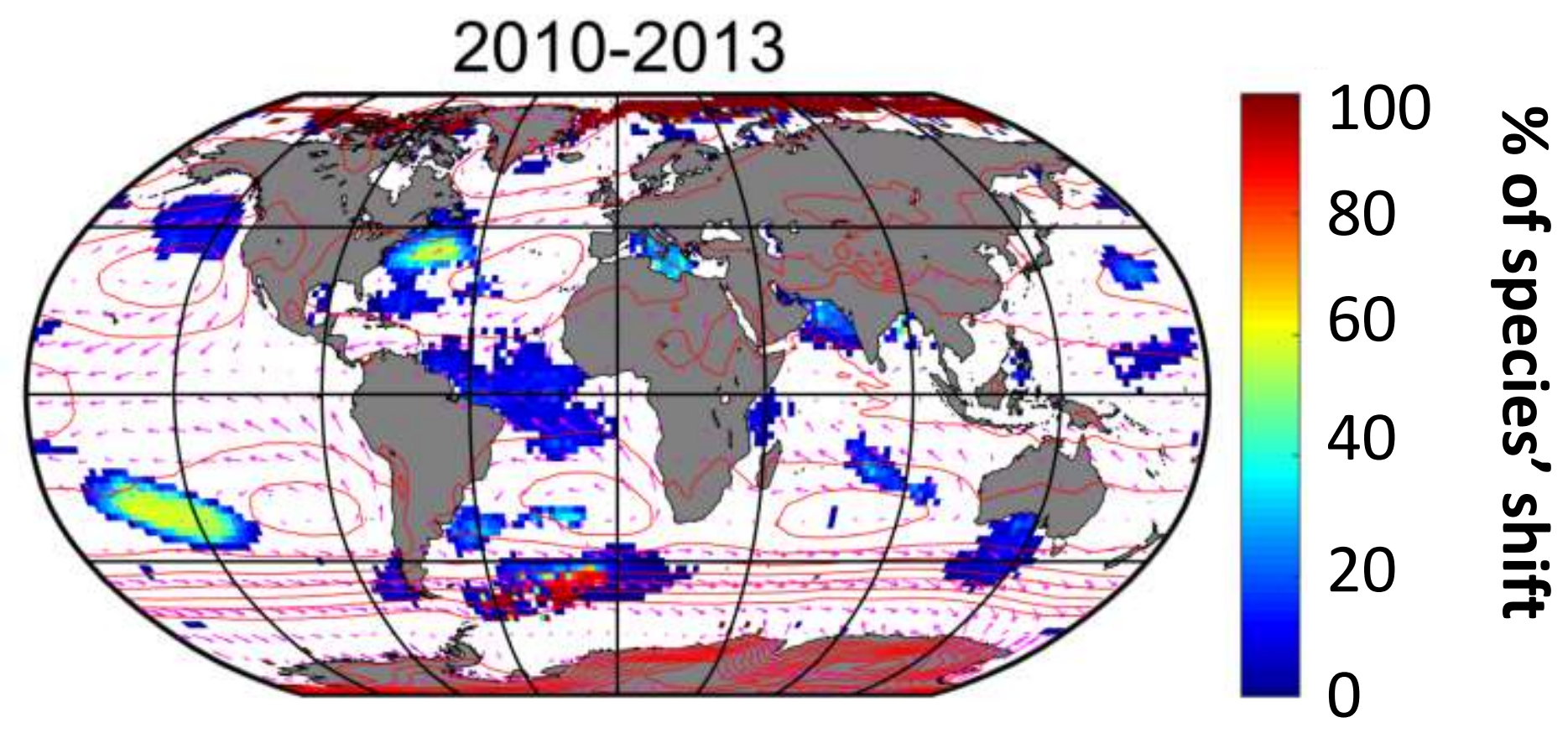

Pink arrow: direction and strengh of mean annual wind (1960-2015) Red line: isobar based on annual SLP for the period 1960-2015 White area: no significant species' shift (threshold $>3$ ) 


\section{Percentage of species' shift into a community}

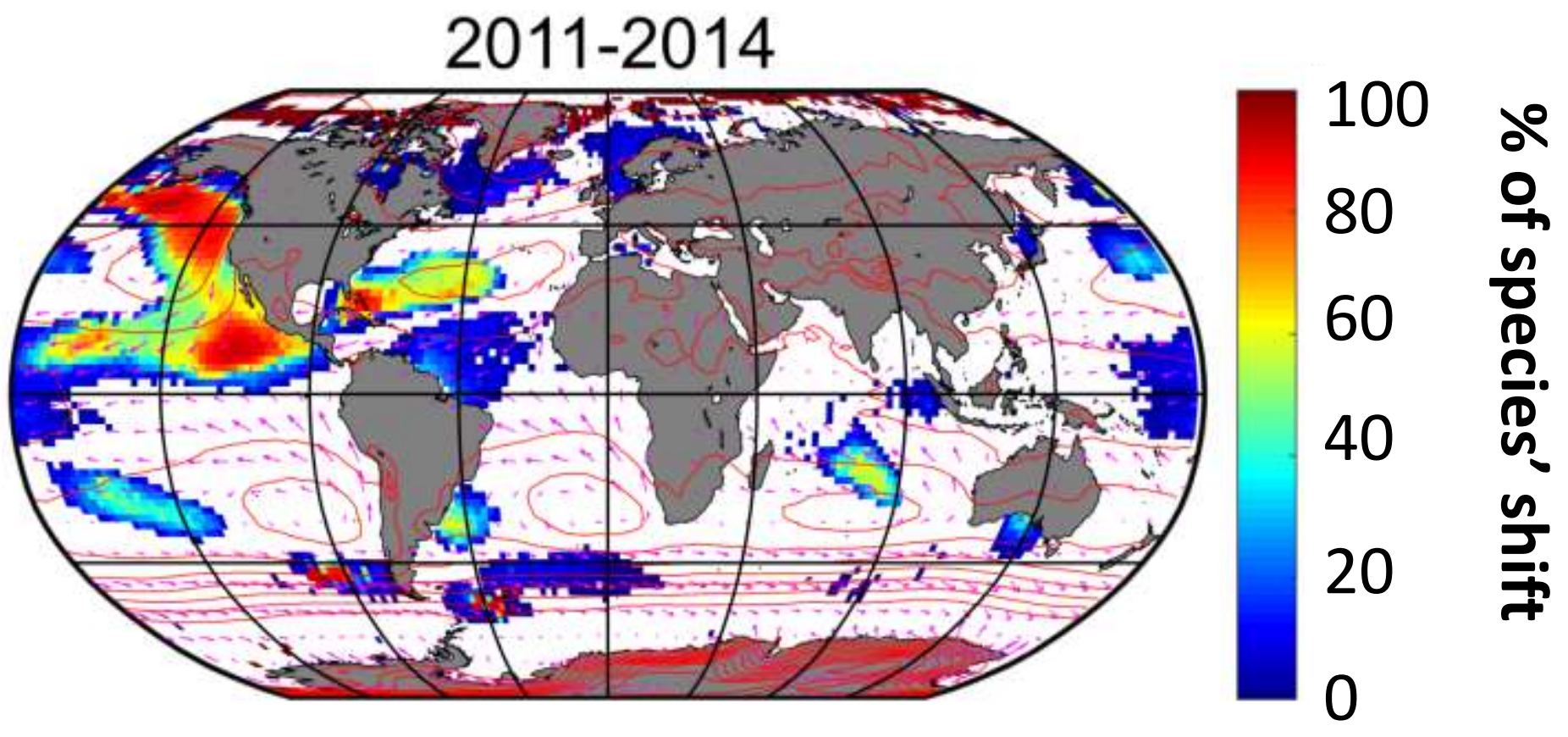

Pink arrow: direction and strengh of mean annual wind (1960-2015) Red line: isobar based on annual SLP for the period 1960-2015 White area: no significant species' shift (threshold $>3$ ) 


\section{Percentage of species' shift into a community}

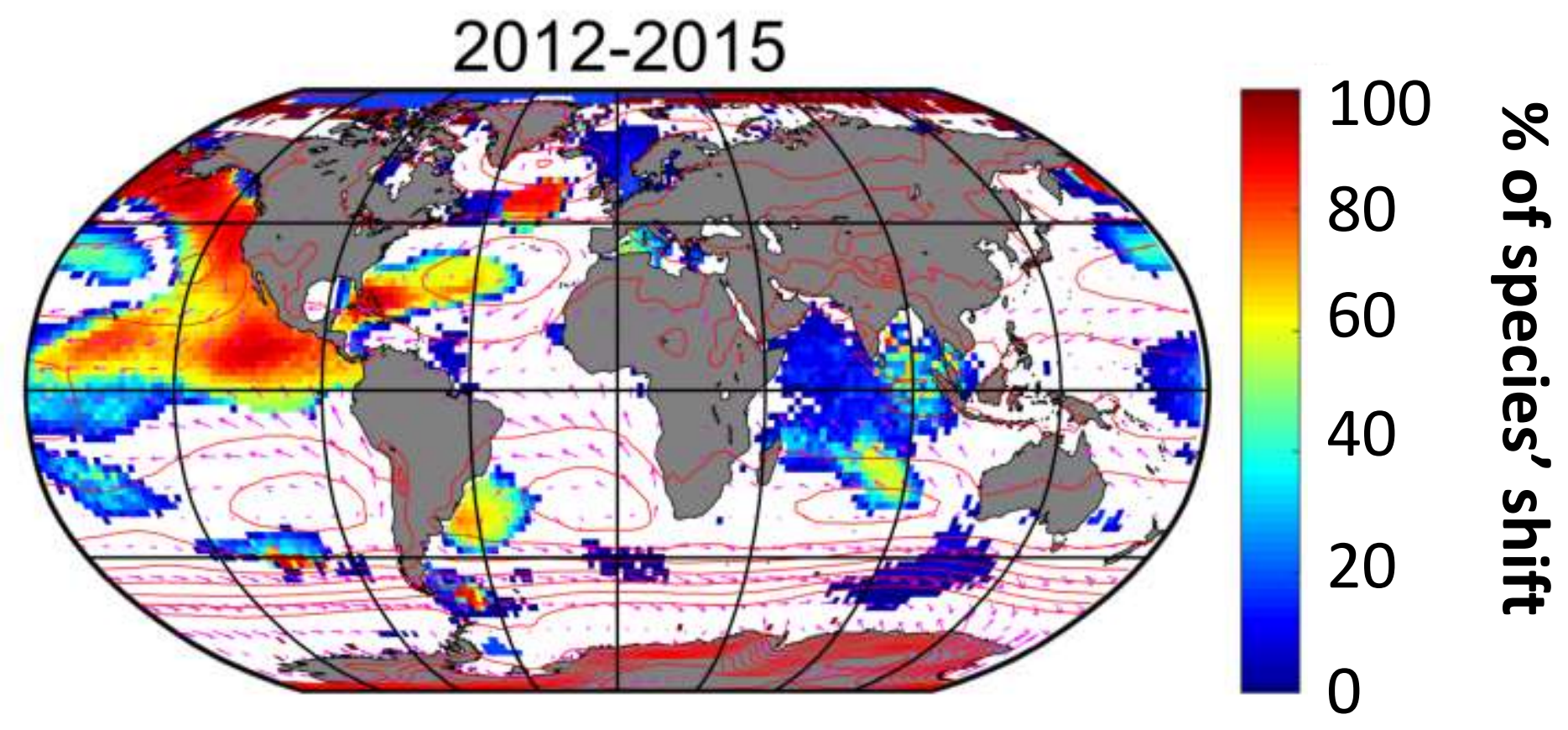

Pink arrow: direction and strengh of mean annual wind (1960-2015) Red line: isobar based on annual SLP for the period 1960-2015 White area: no significant species' shift (threshold $>3$ ) 


\section{Supplementary Figure 5}

\section{Number of climatic shifts in each geographical cell}

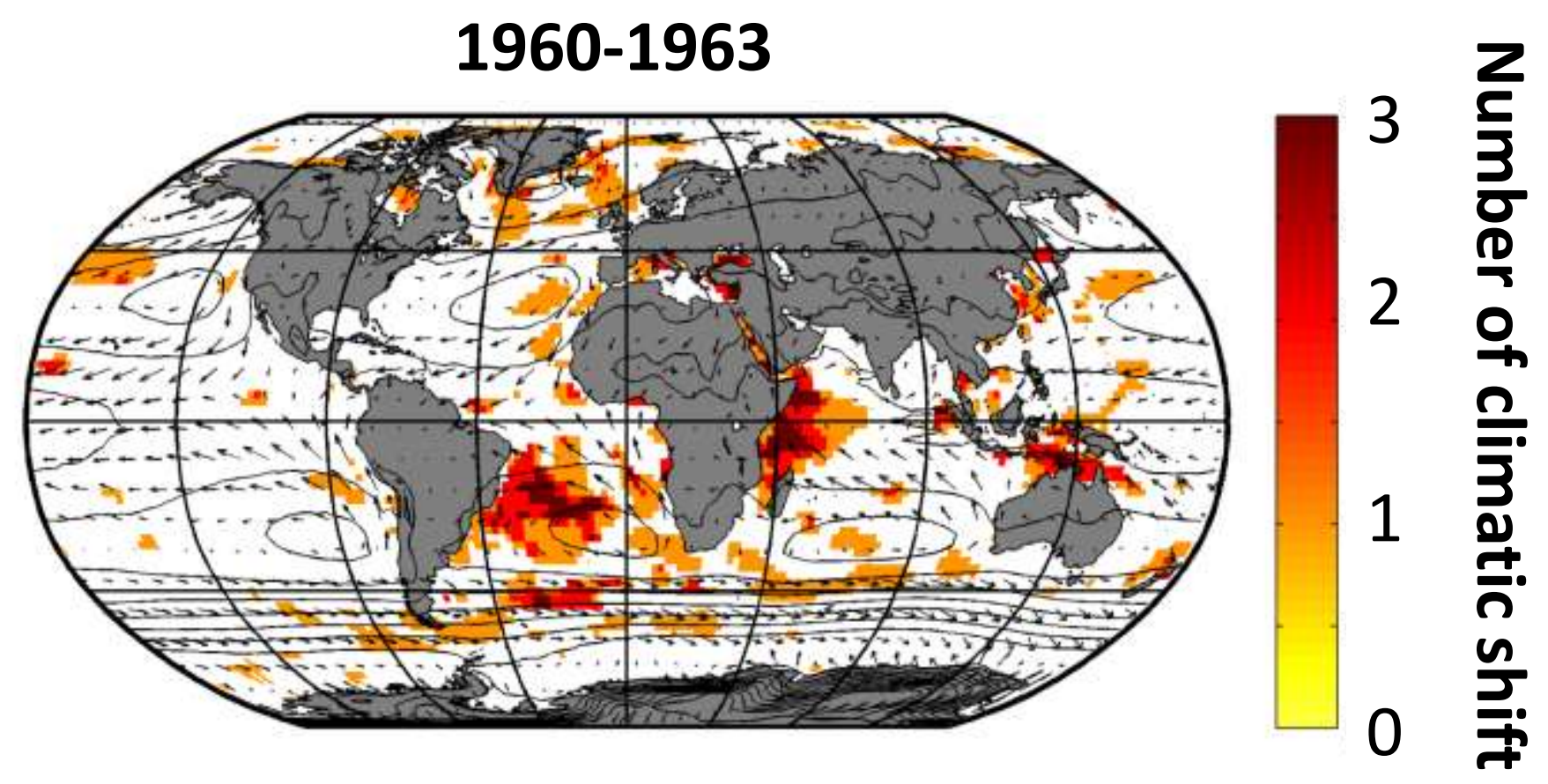

Black arrow: direction and strengh of mean annual wind (1960-2015) Red line: isobar based on annual SLP for the period 1960-2015 White area: no significant species' shift (threshold $>3$ )

Six climatic parameters were used: Annual sea level pressure, wind intensity, meridional and zonal wind, cloudiness, sea surface temperature. 


\section{Supplementary Figure 5}

\section{Number of climatic shifts in each geographical cell}

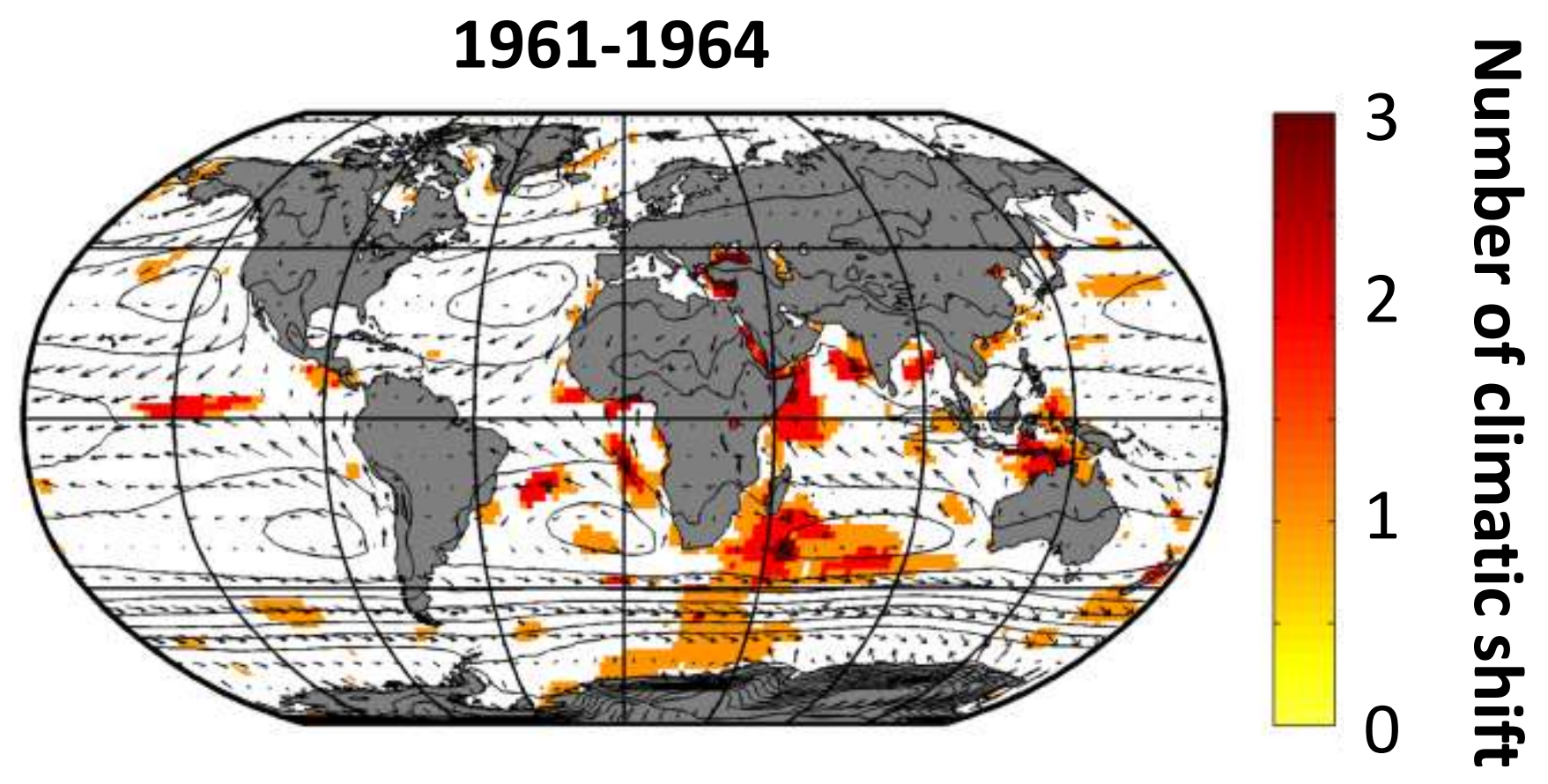

Black arrow: direction and strengh of mean annual wind (1960-2015) Red line: isobar based on annual SLP for the period 1960-2015 White area: no significant species' shift (threshold $>3$ )

Six climatic parameters were used: Annual sea level pressure, wind intensity, meridional and zonal wind, cloudiness, sea surface temperature. 


\section{Supplementary Figure 5}

\section{Number of climatic shifts in each geographical cell}

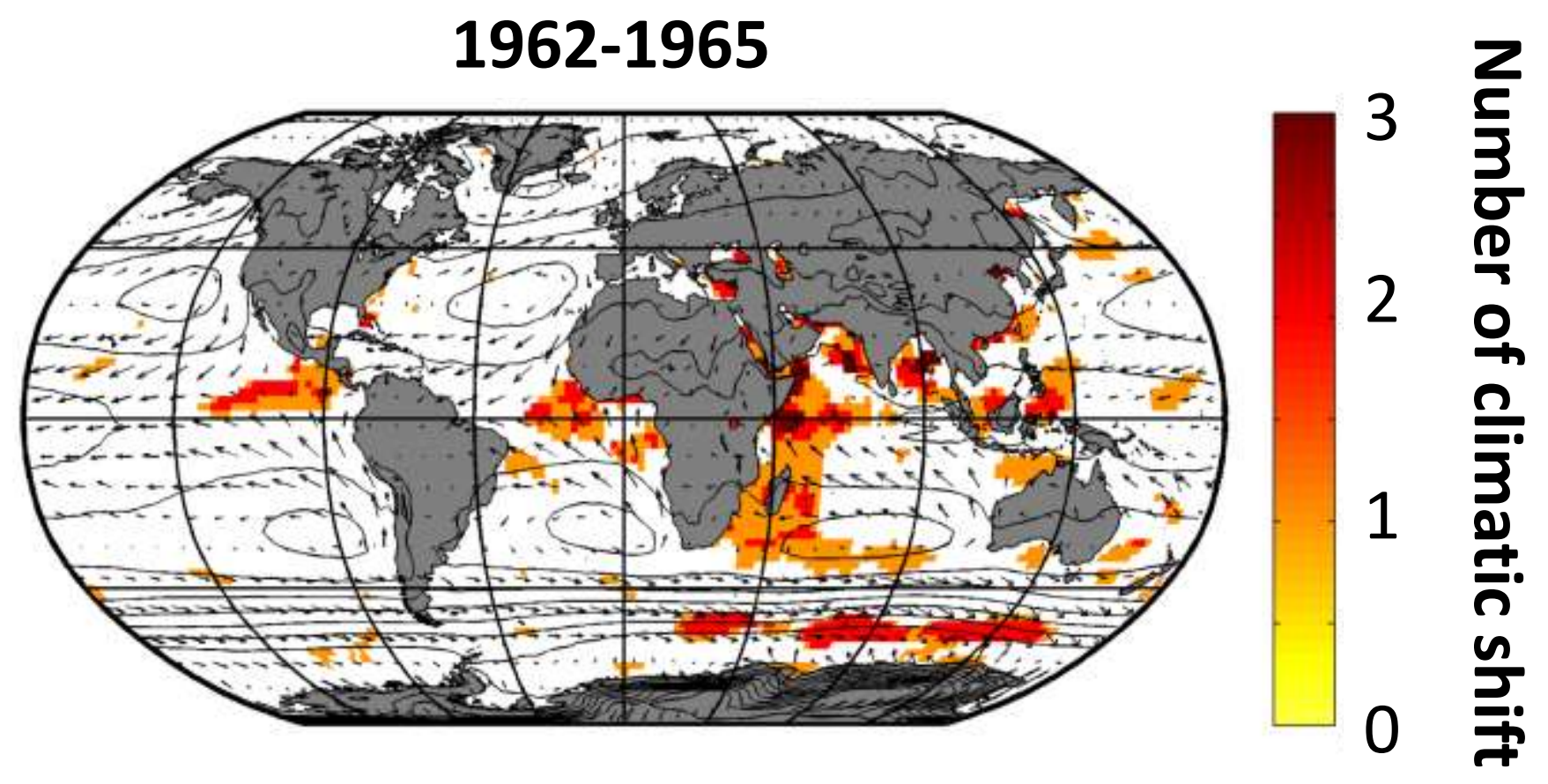

Black arrow: direction and strengh of mean annual wind (1960-2015) Red line: isobar based on annual SLP for the period 1960-2015 White area: no significant species' shift (threshold $>3$ )

Six climatic parameters were used: Annual sea level pressure, wind intensity, meridional and zonal wind, cloudiness, sea surface temperature. 


\section{Supplementary Figure 5}

\section{Number of climatic shifts in each geographical cell}

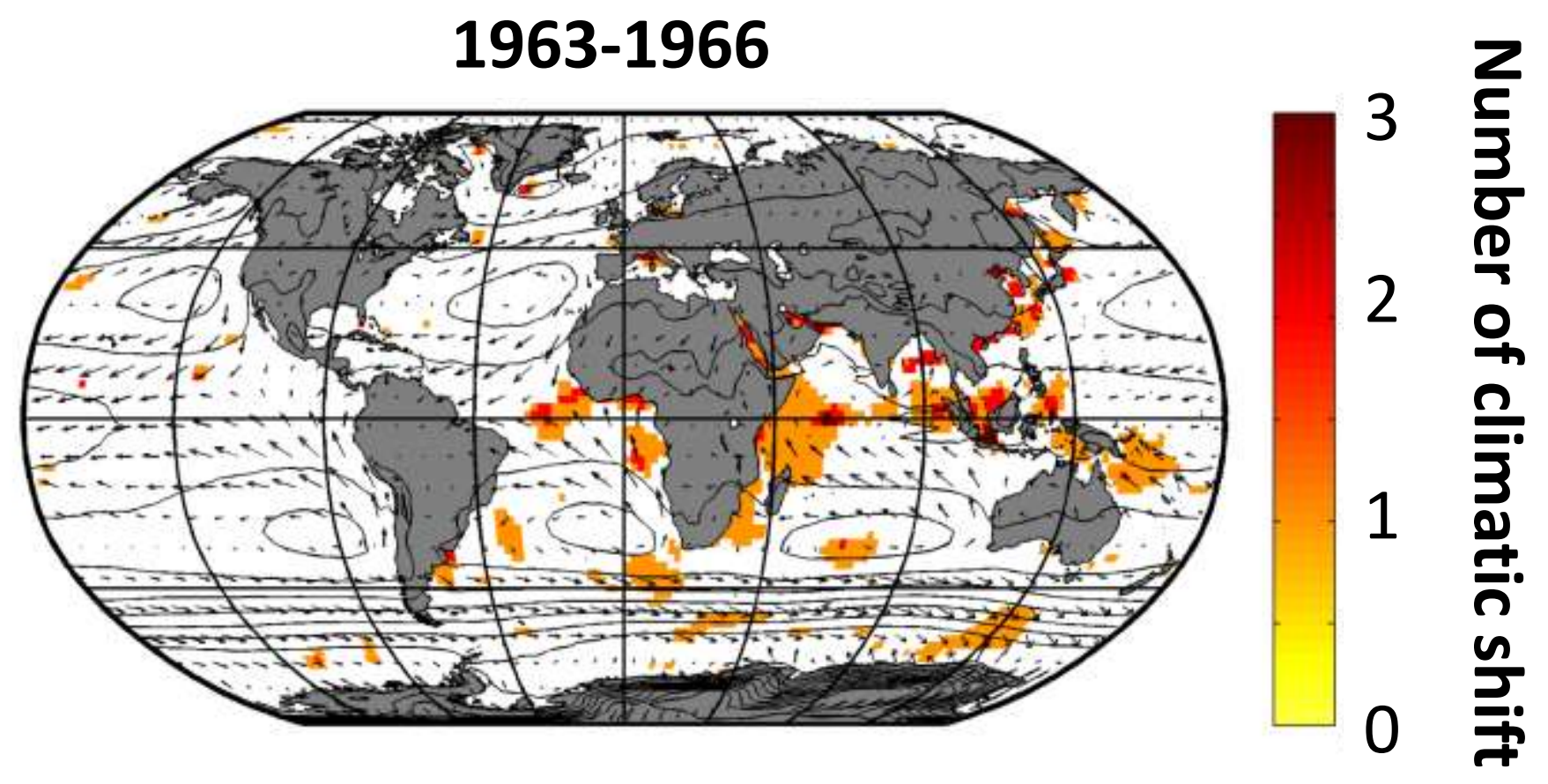

Black arrow: direction and strengh of mean annual wind (1960-2015) Red line: isobar based on annual SLP for the period 1960-2015 White area: no significant species' shift (threshold $>3$ )

Six climatic parameters were used: Annual sea level pressure, wind intensity, meridional and zonal wind, cloudiness, sea surface temperature. 


\section{Supplementary Figure 5}

\section{Number of climatic shifts in each geographical cell}

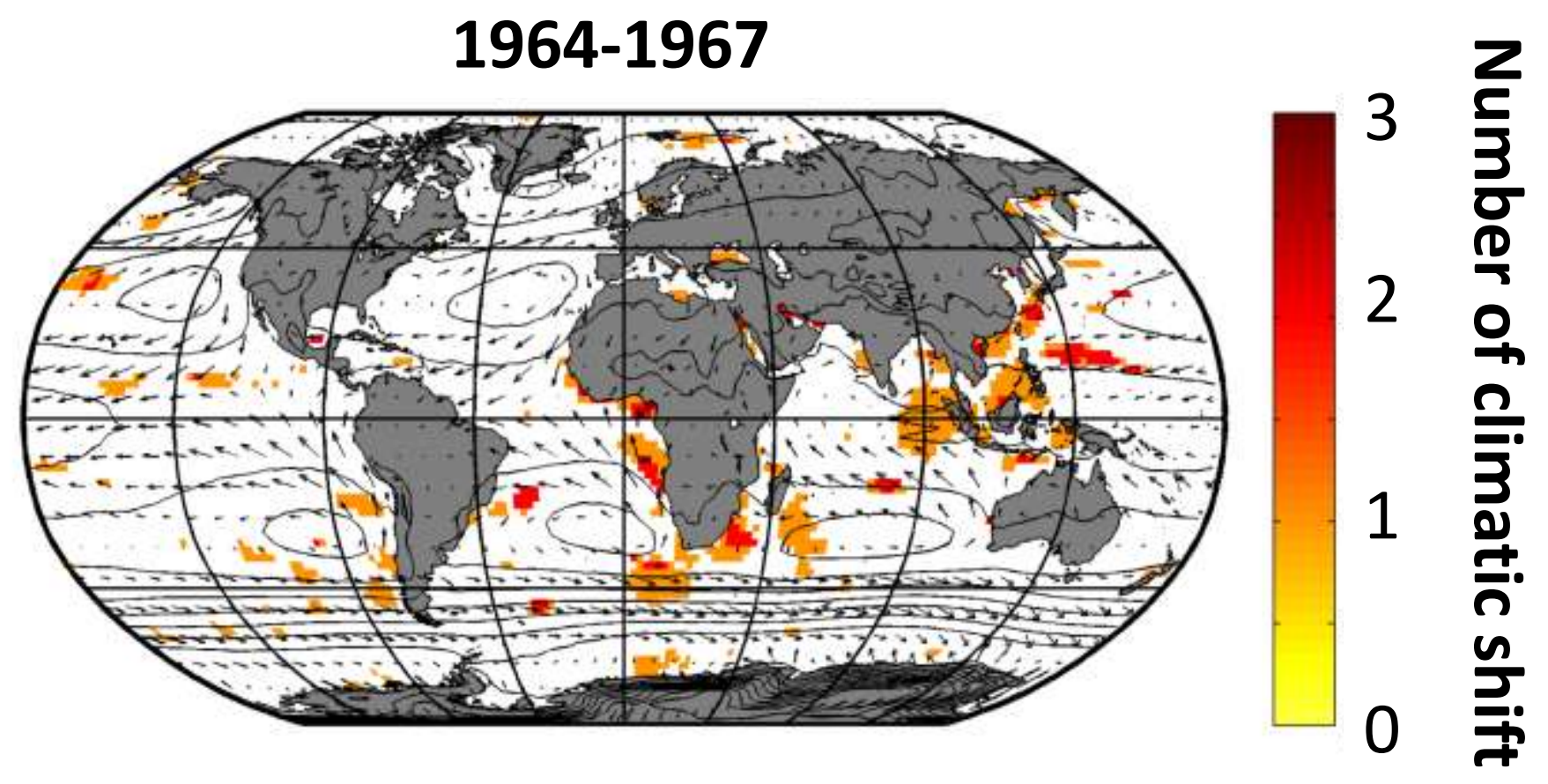

Black arrow: direction and strengh of mean annual wind (1960-2015) Red line: isobar based on annual SLP for the period 1960-2015 White area: no significant species' shift (threshold $>3$ )

Six climatic parameters were used: Annual sea level pressure, wind intensity, meridional and zonal wind, cloudiness, sea surface temperature. 


\section{Supplementary Figure 5}

\section{Number of climatic shifts in each geographical cell}

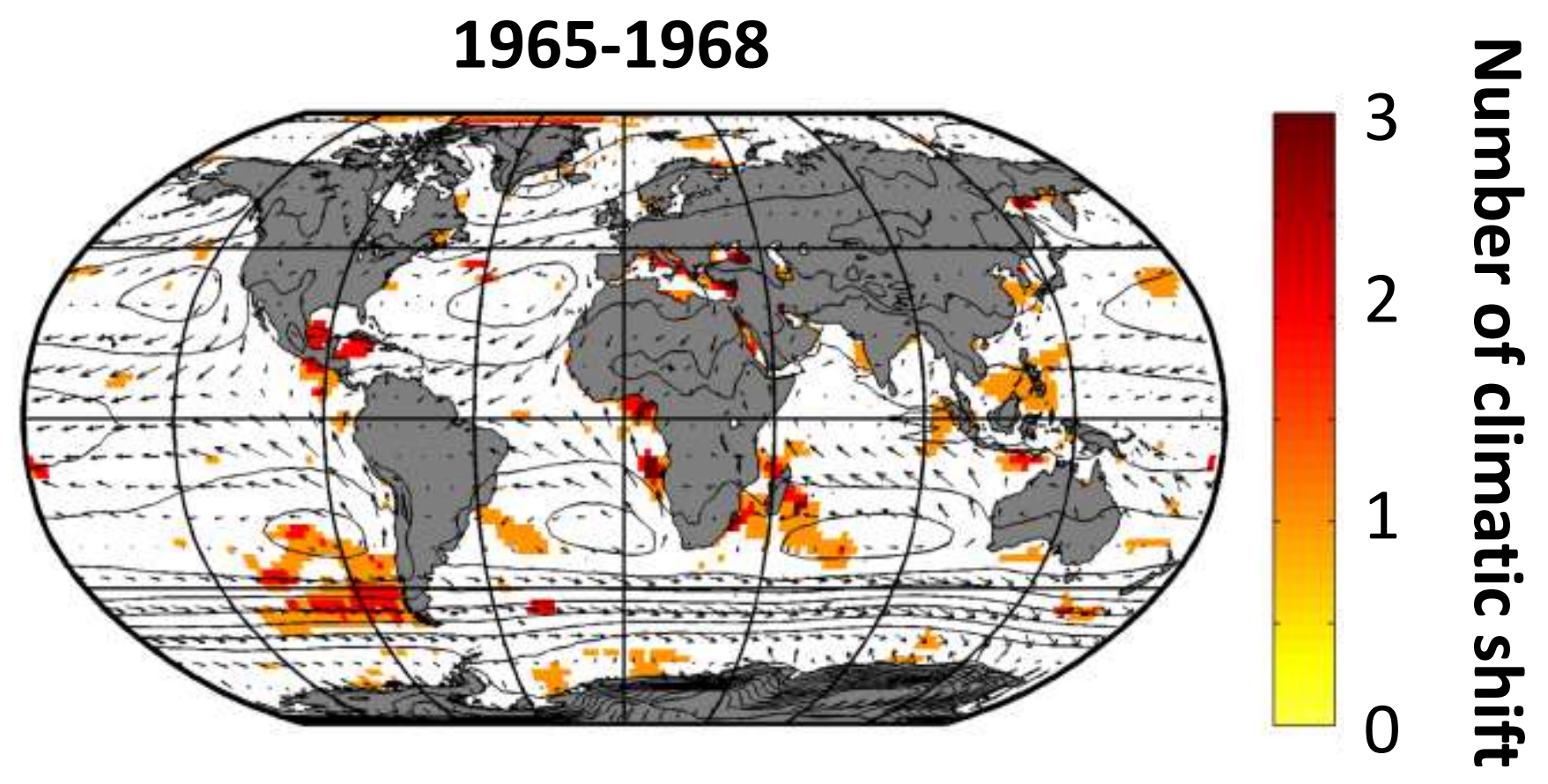

Black arrow: direction and strengh of mean annual wind (1960-2015) Red line: isobar based on annual SLP for the period 1960-2015 White area: no significant species' shift (threshold $>3$ )

Six climatic parameters were used: Annual sea level pressure, wind intensity, meridional and zonal wind, cloudiness, sea surface temperature. 


\section{Supplementary Figure 5}

\section{Number of climatic shifts in each geographical cell}

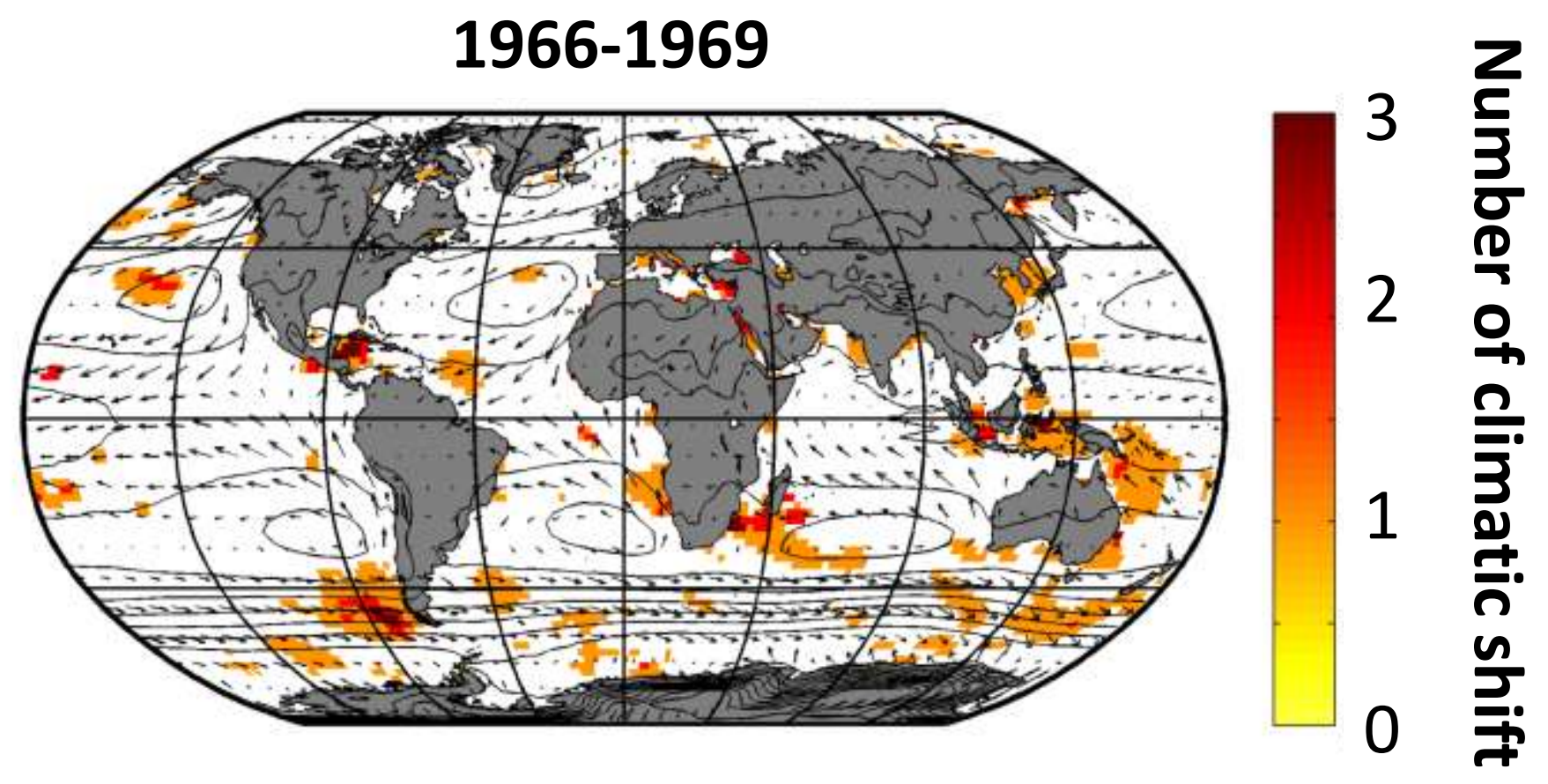

Black arrow: direction and strengh of mean annual wind (1960-2015) Red line: isobar based on annual SLP for the period 1960-2015 White area: no significant species' shift (threshold $>3$ )

Six climatic parameters were used: Annual sea level pressure, wind intensity, meridional and zonal wind, cloudiness, sea surface temperature. 


\section{Supplementary Figure 5}

\section{Number of climatic shifts in each geographical cell}

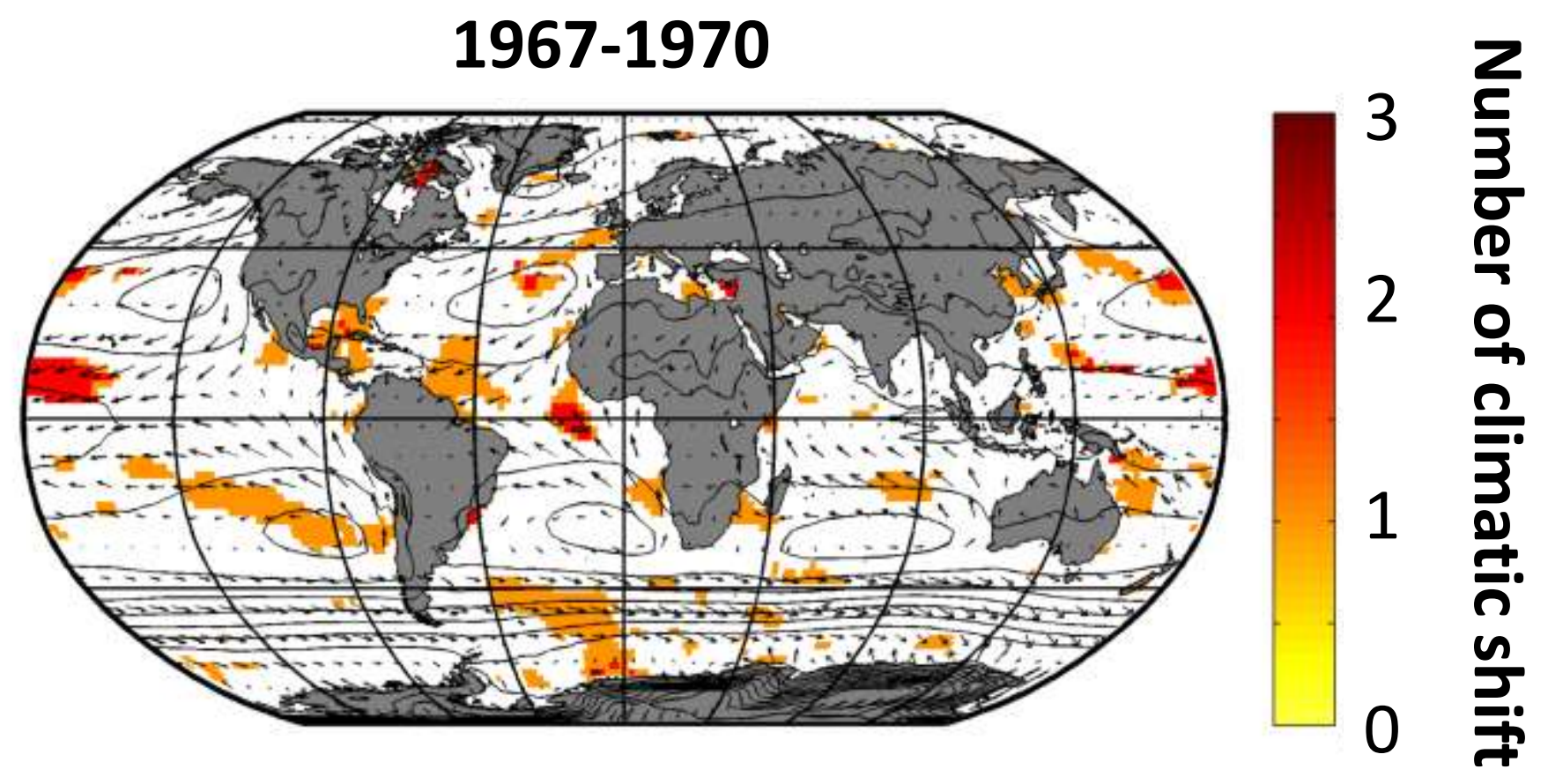

Black arrow: direction and strengh of mean annual wind (1960-2015) Red line: isobar based on annual SLP for the period 1960-2015 White area: no significant species' shift (threshold $>3$ )

Six climatic parameters were used: Annual sea level pressure, wind intensity, meridional and zonal wind, cloudiness, sea surface temperature. 


\section{Supplementary Figure 5}

\section{Number of climatic shifts in each geographical cell}

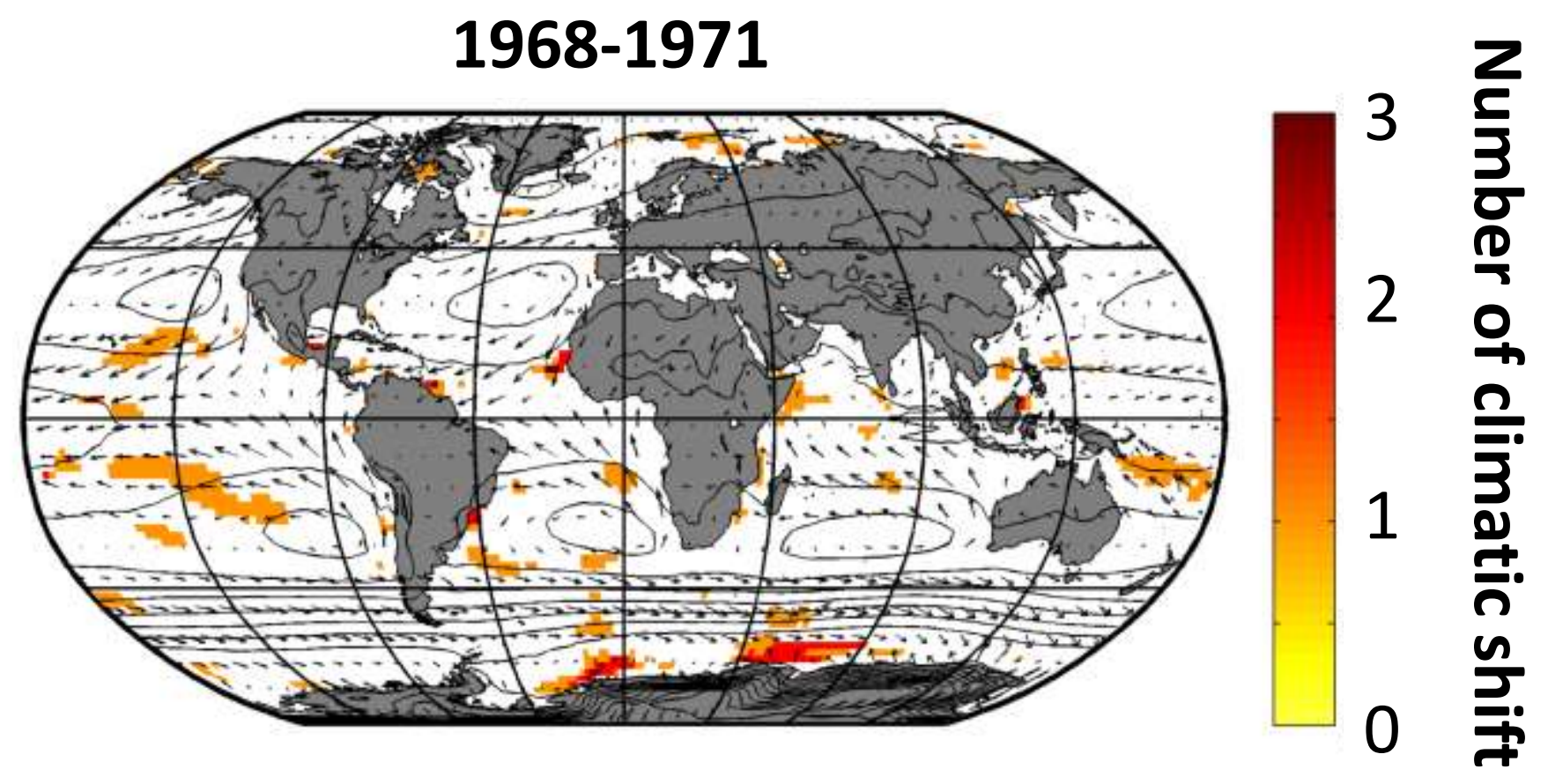

Black arrow: direction and strengh of mean annual wind (1960-2015) Red line: isobar based on annual SLP for the period 1960-2015 White area: no significant species' shift (threshold $>3$ )

Six climatic parameters were used: Annual sea level pressure, wind intensity, meridional and zonal wind, cloudiness, sea surface temperature. 


\section{Supplementary Figure 5}

\section{Number of climatic shifts in each geographical cell}

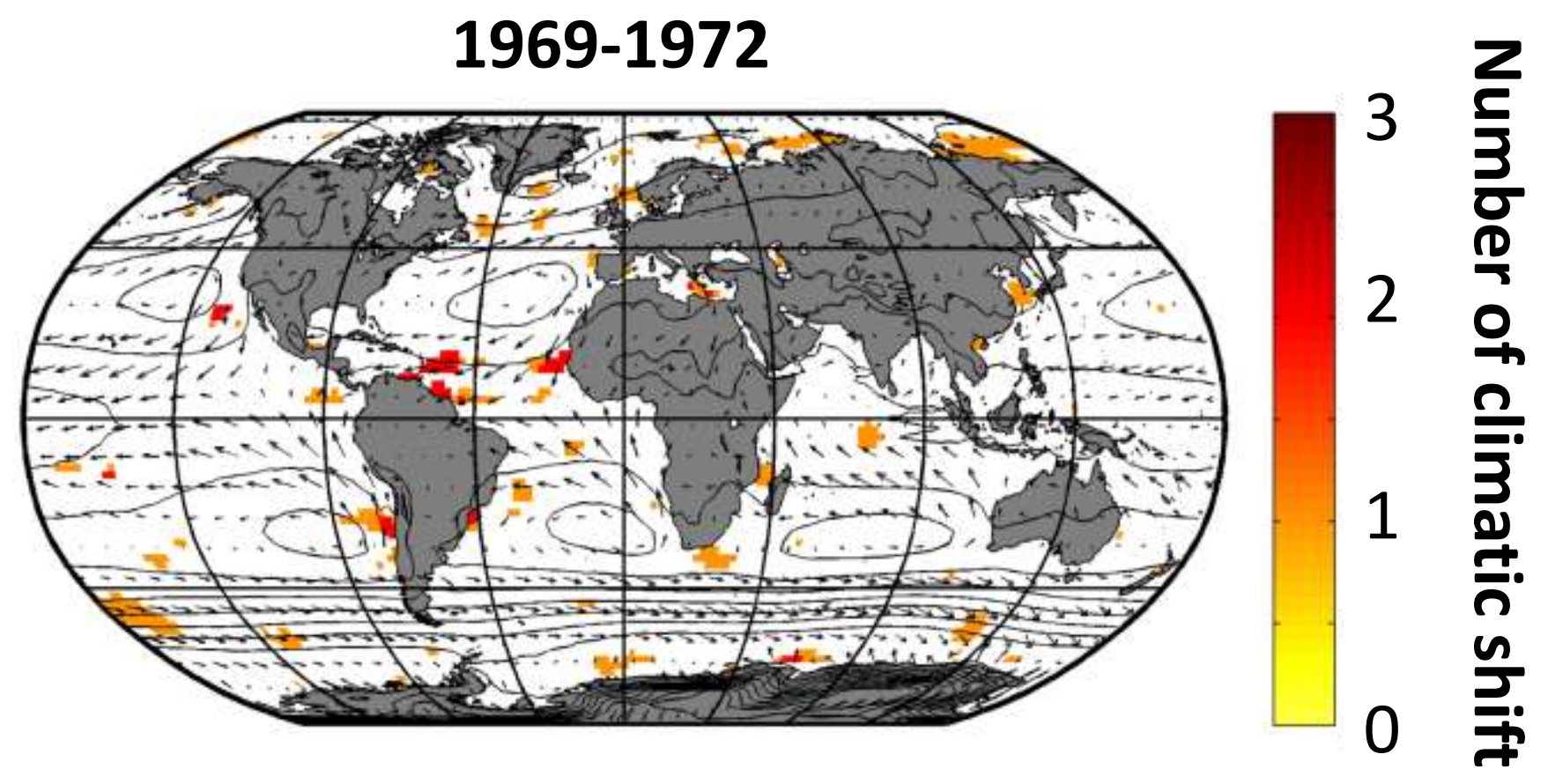

Black arrow: direction and strengh of mean annual wind (1960-2015) Red line: isobar based on annual SLP for the period 1960-2015 White area: no significant species' shift (threshold $>3$ )

Six climatic parameters were used: Annual sea level pressure, wind intensity, meridional and zonal wind, cloudiness, sea surface temperature. 


\section{Supplementary Figure 5}

\section{Number of climatic shifts in each geographical cell}

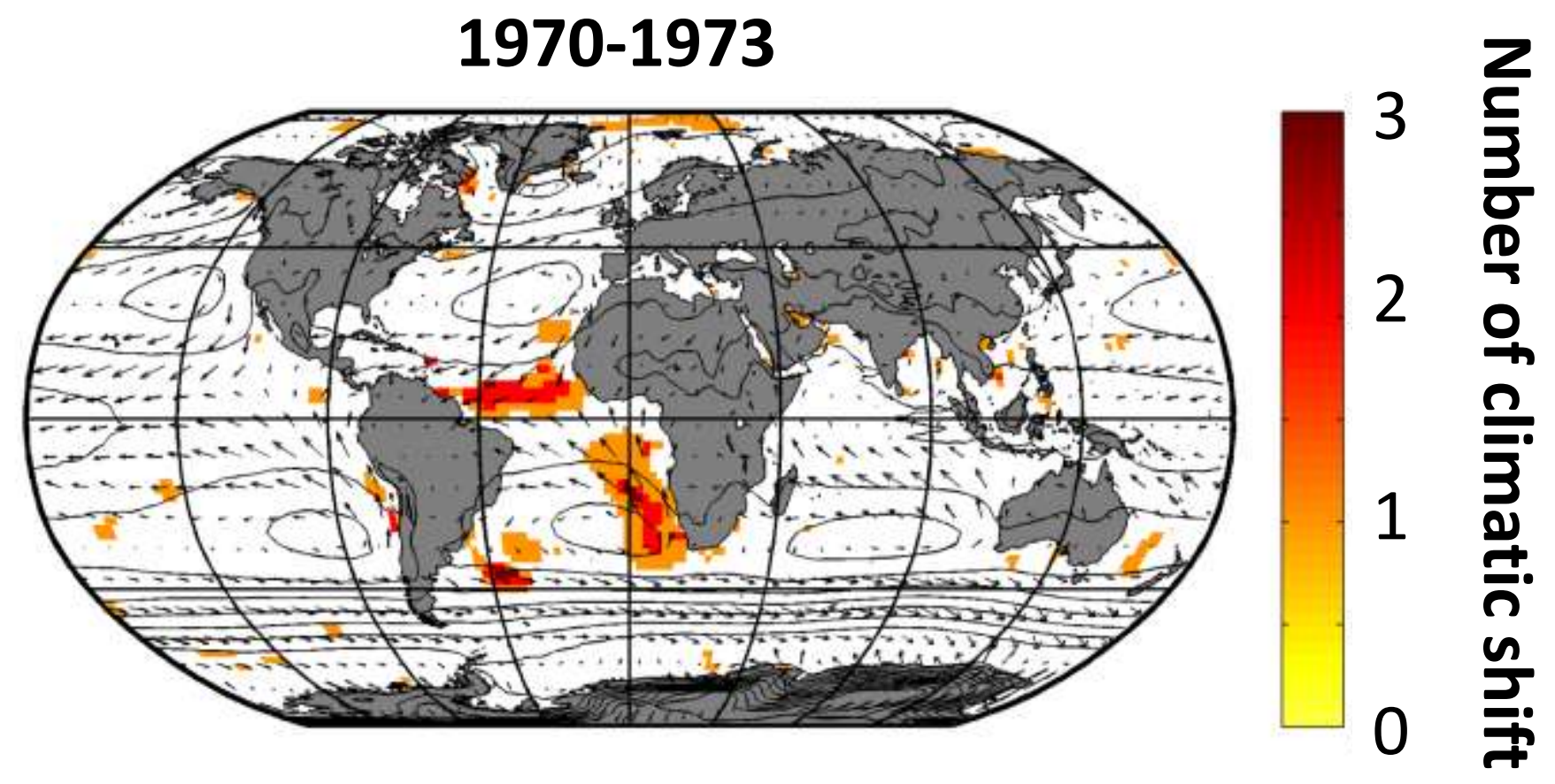

Black arrow: direction and strengh of mean annual wind (1960-2015) Red line: isobar based on annual SLP for the period 1960-2015 White area: no significant species' shift (threshold $>3$ )

Six climatic parameters were used: Annual sea level pressure, wind intensity, meridional and zonal wind, cloudiness, sea surface temperature. 


\section{Supplementary Figure 5}

\section{Number of climatic shifts in each geographical cell}

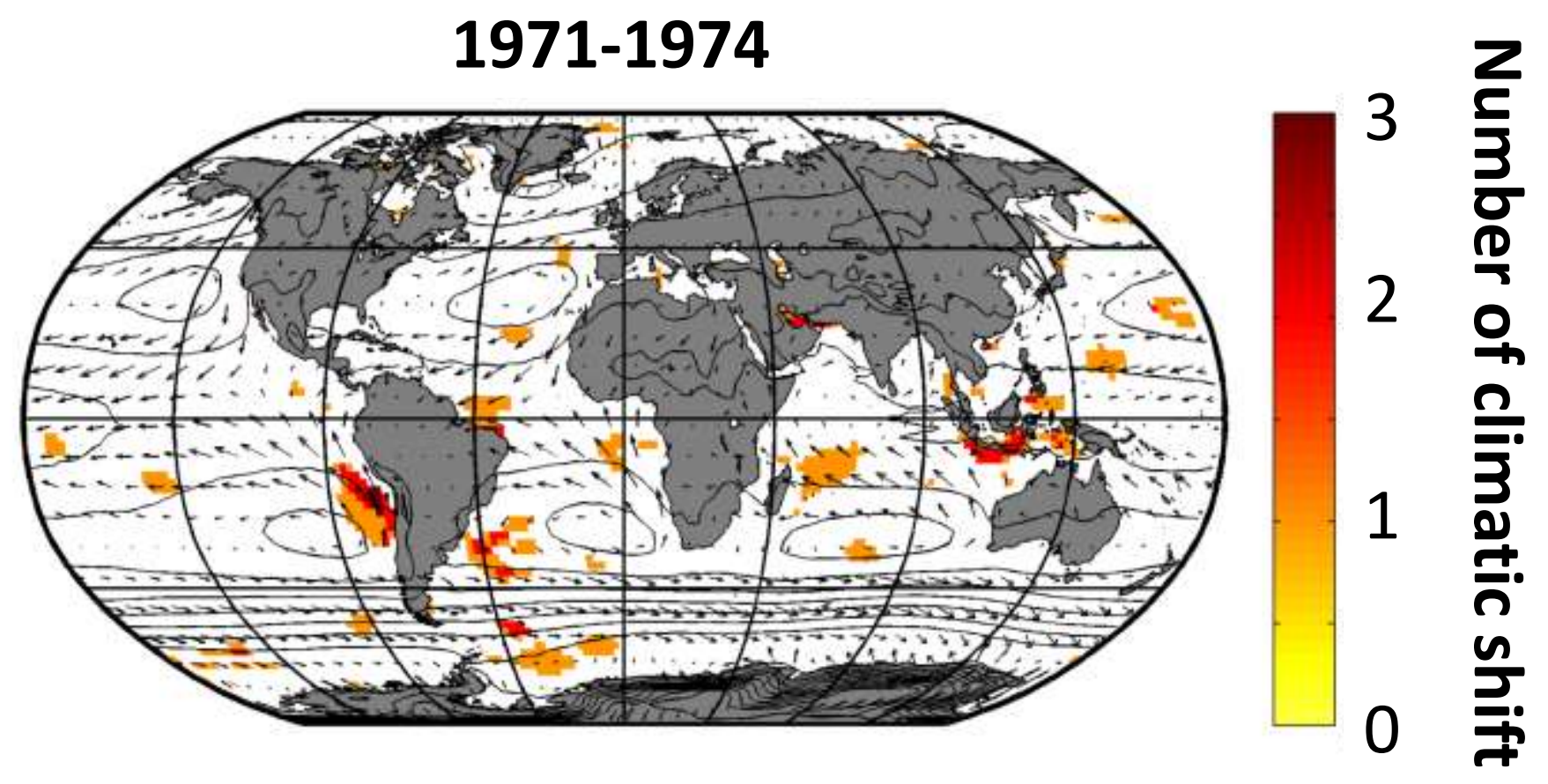

Black arrow: direction and strengh of mean annual wind (1960-2015) Red line: isobar based on annual SLP for the period 1960-2015 White area: no significant species' shift (threshold $>3$ )

Six climatic parameters were used: Annual sea level pressure, wind intensity, meridional and zonal wind, cloudiness, sea surface temperature. 


\section{Supplementary Figure 5}

\section{Number of climatic shifts in each geographical cell}

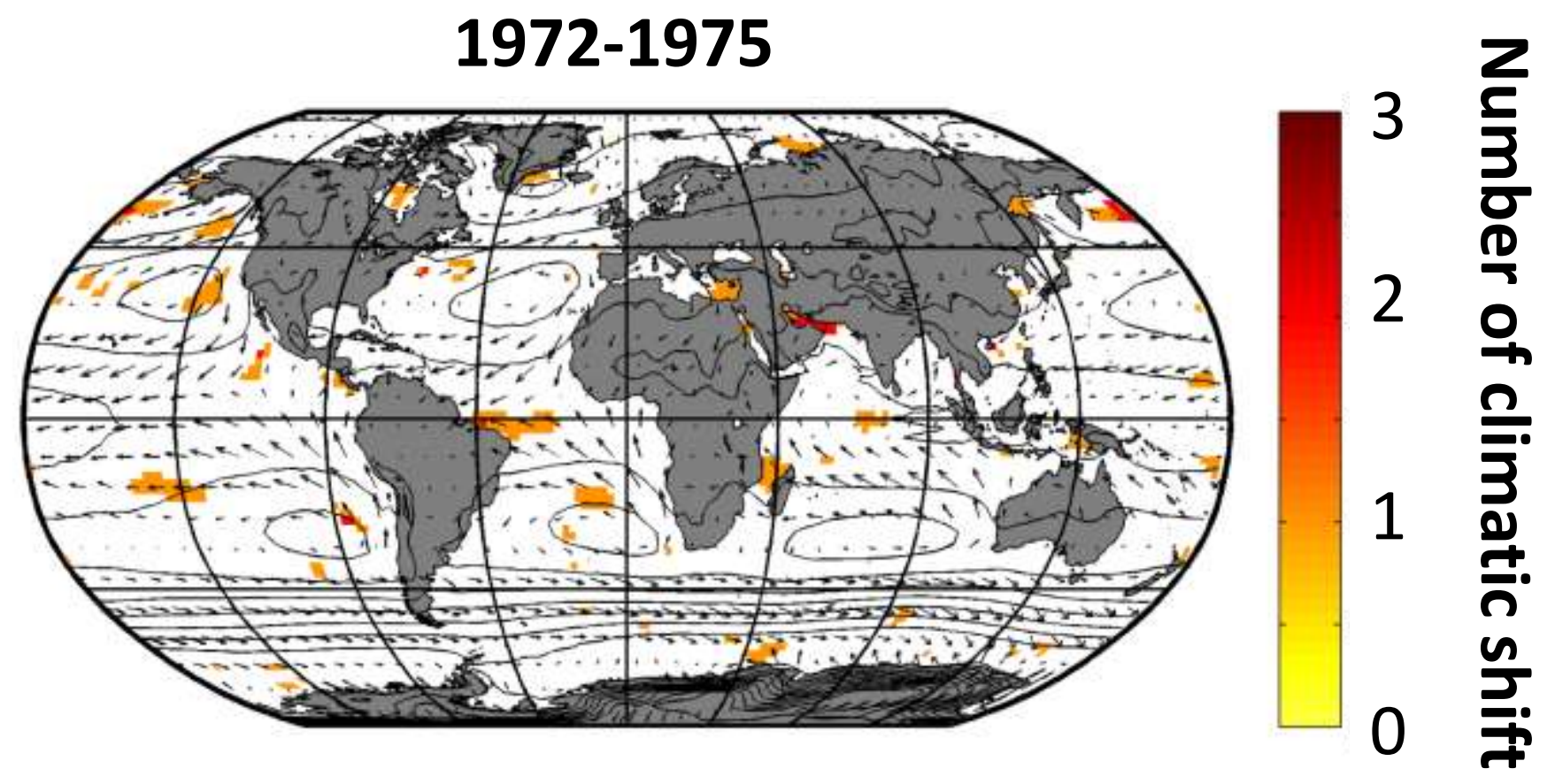

Black arrow: direction and strengh of mean annual wind (1960-2015) Red line: isobar based on annual SLP for the period 1960-2015 White area: no significant species' shift (threshold $>3$ )

Six climatic parameters were used: Annual sea level pressure, wind intensity, meridional and zonal wind, cloudiness, sea surface temperature. 


\section{Supplementary Figure 5}

\section{Number of climatic shifts in each geographical cell}

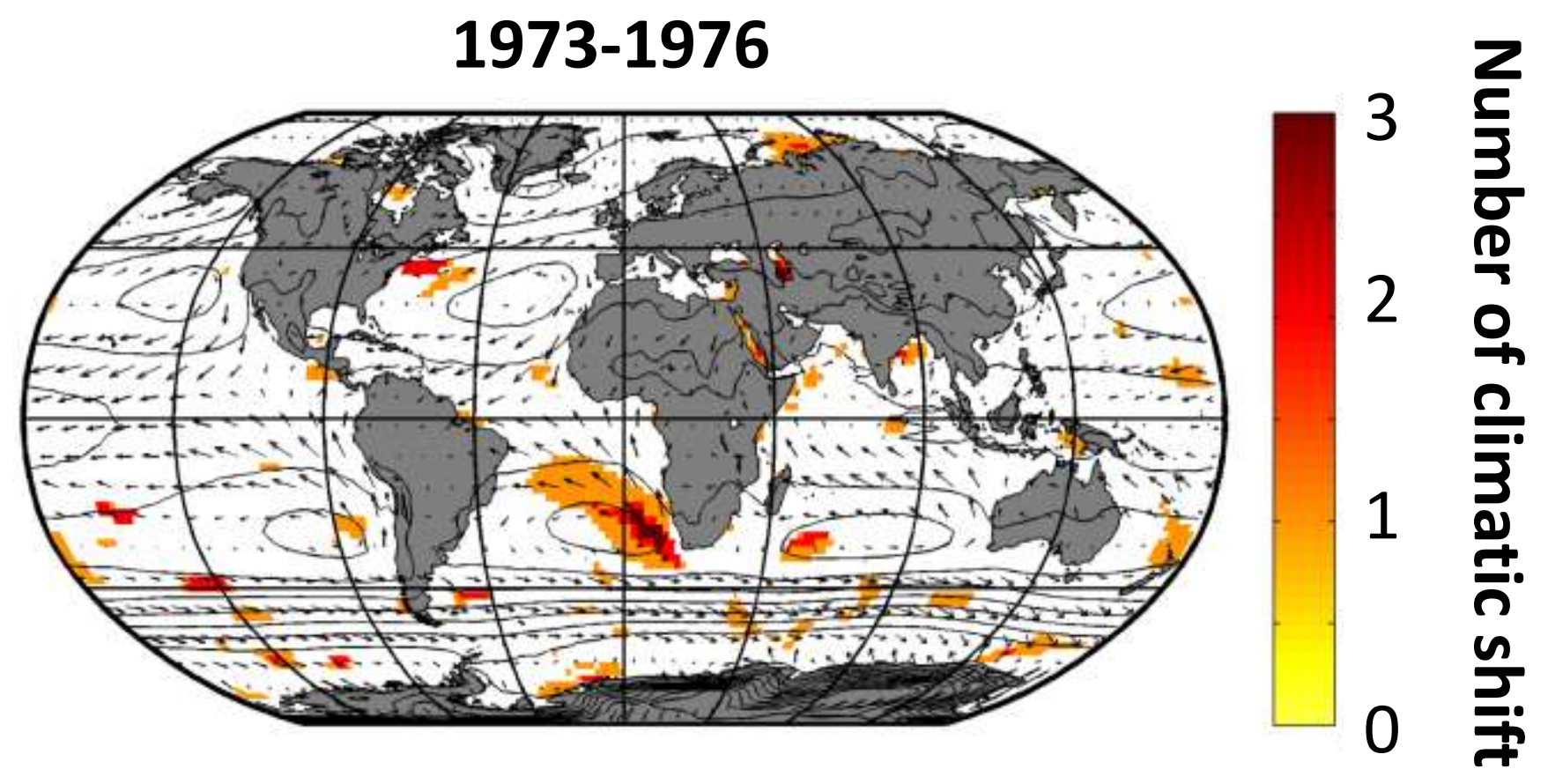

Black arrow: direction and strengh of mean annual wind (1960-2015) Red line: isobar based on annual SLP for the period 1960-2015 White area: no significant species' shift (threshold $>3$ )

Six climatic parameters were used: Annual sea level pressure, wind intensity, meridional and zonal wind, cloudiness, sea surface temperature. 


\section{Supplementary Figure 5}

\section{Number of climatic shifts in each geographical cell}

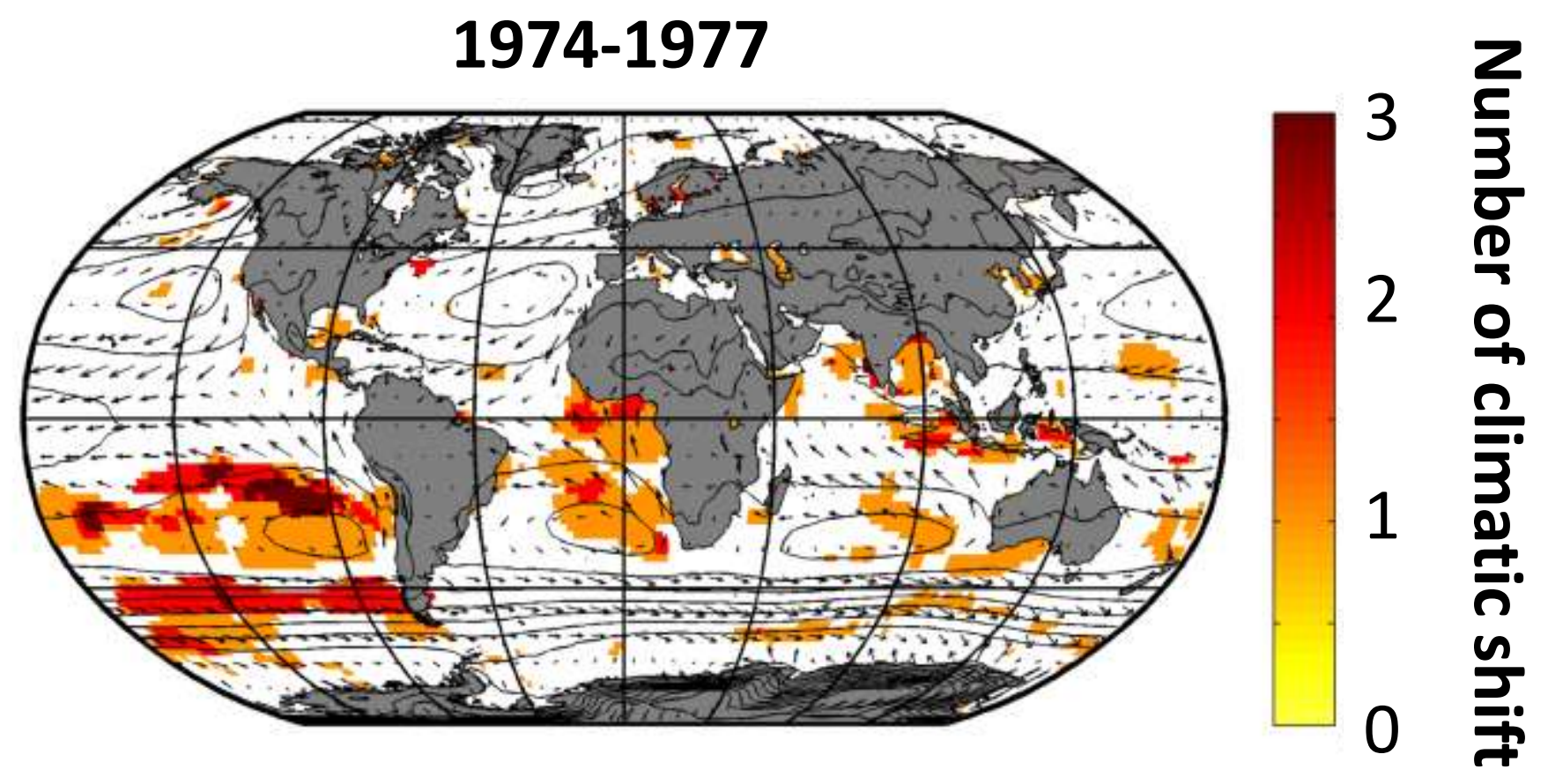

Black arrow: direction and strengh of mean annual wind (1960-2015) Red line: isobar based on annual SLP for the period 1960-2015 White area: no significant species' shift (threshold $>3$ )

Six climatic parameters were used: Annual sea level pressure, wind intensity, meridional and zonal wind, cloudiness, sea surface temperature. 


\section{Supplementary Figure 5}

\section{Number of climatic shifts in each geographical cell}

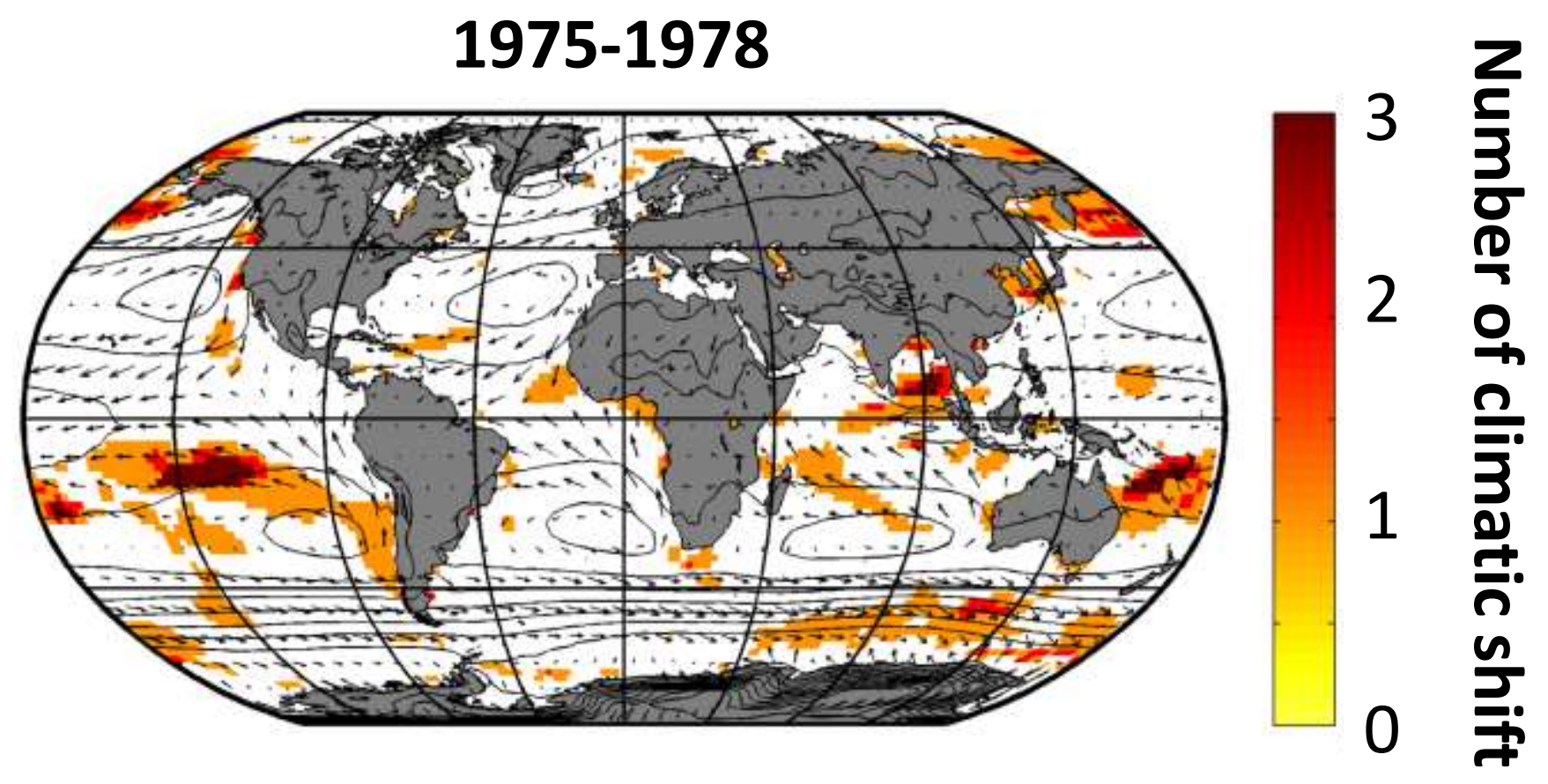

Black arrow: direction and strengh of mean annual wind (1960-2015) Red line: isobar based on annual SLP for the period 1960-2015 White area: no significant species' shift (threshold $>3$ )

Six climatic parameters were used: Annual sea level pressure, wind intensity, meridional and zonal wind, cloudiness, sea surface temperature. 


\section{Supplementary Figure 5}

\section{Number of climatic shifts in each geographical cell}

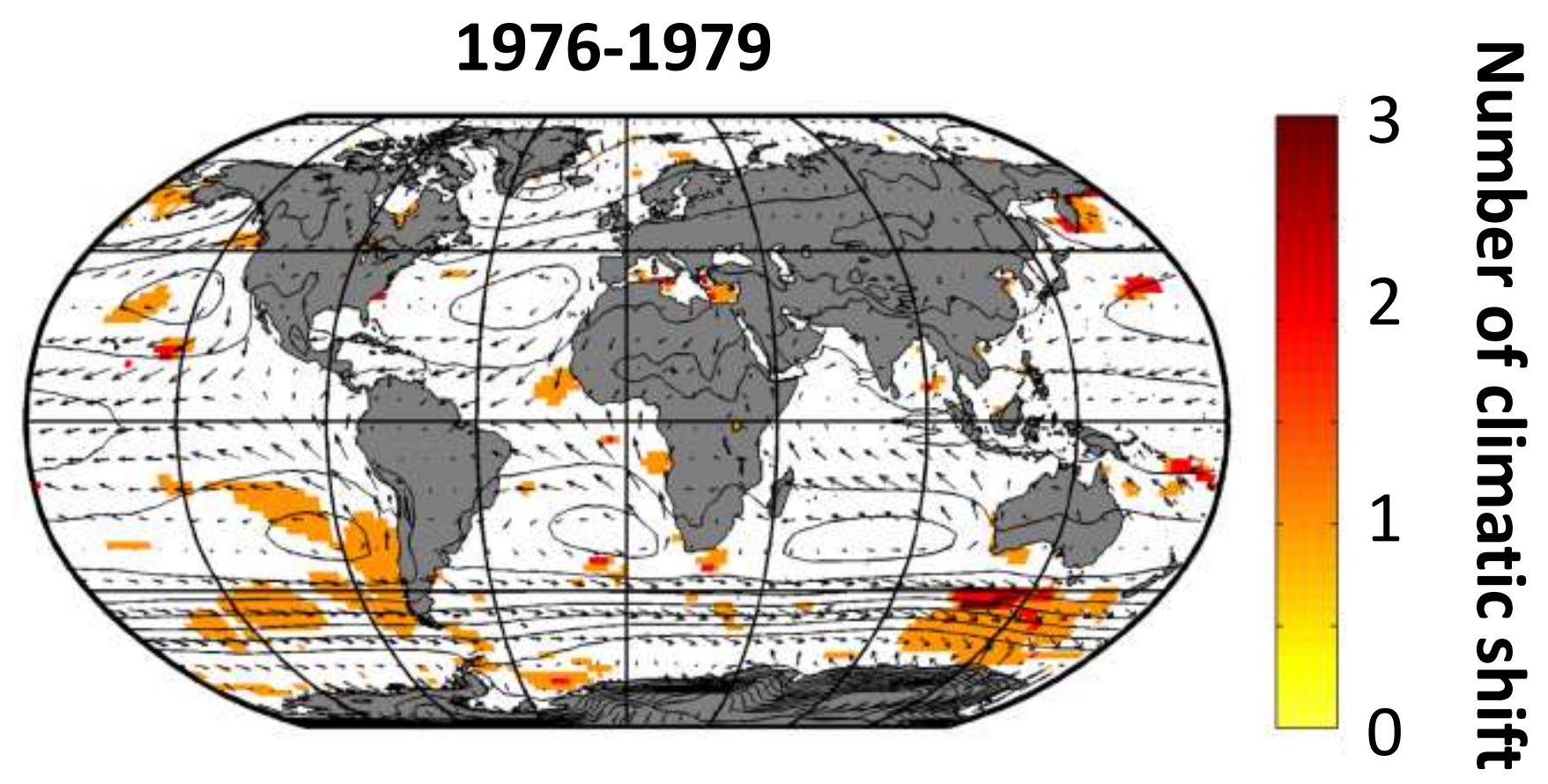

Black arrow: direction and strengh of mean annual wind (1960-2015) Red line: isobar based on annual SLP for the period 1960-2015 White area: no significant species' shift (threshold $>3$ )

Six climatic parameters were used: Annual sea level pressure, wind intensity, meridional and zonal wind, cloudiness, sea surface temperature. 


\section{Supplementary Figure 5}

\section{Number of climatic shifts in each geographical cell}

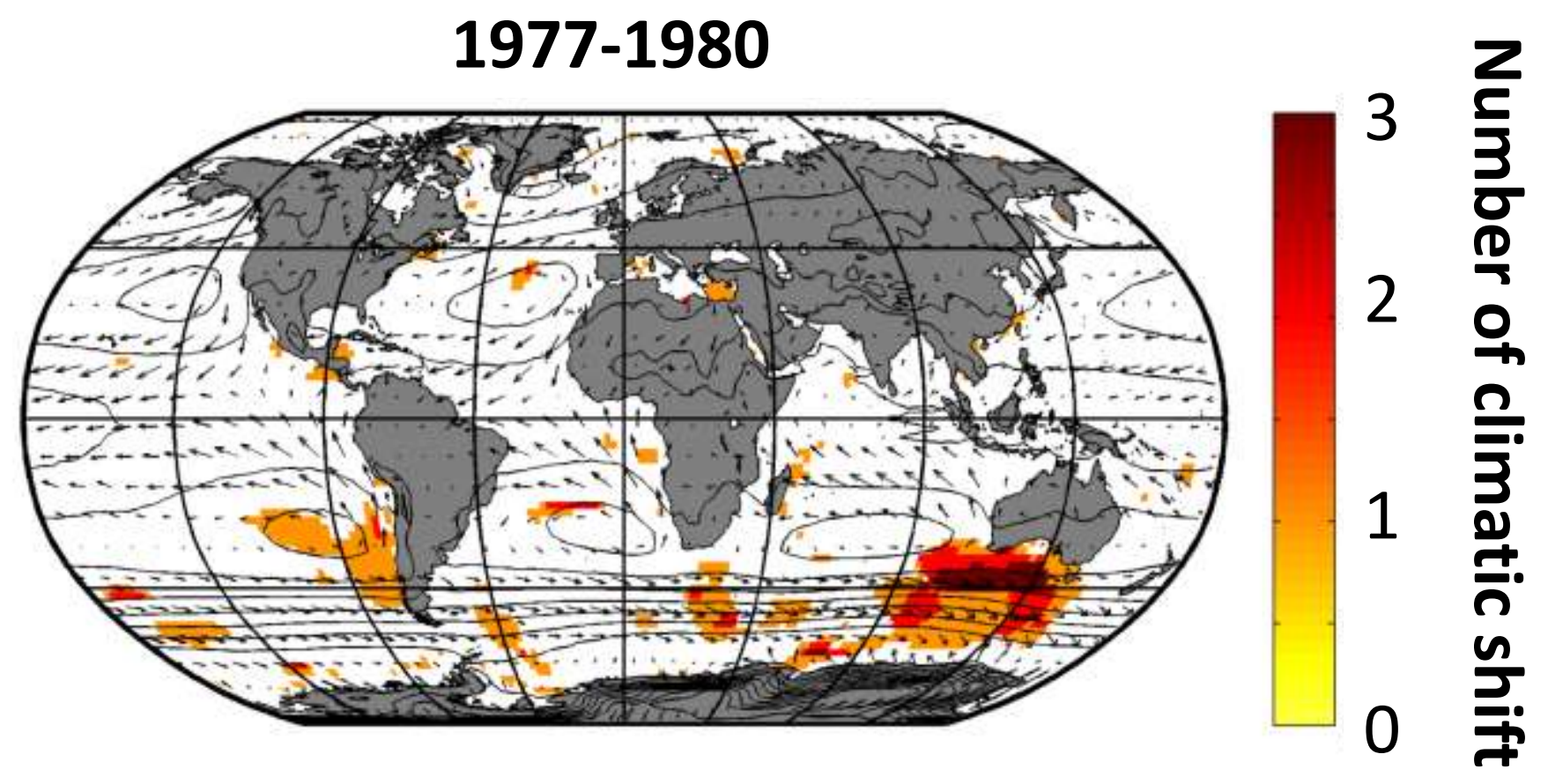

Black arrow: direction and strengh of mean annual wind (1960-2015) Red line: isobar based on annual SLP for the period 1960-2015 White area: no significant species' shift (threshold $>3$ )

Six climatic parameters were used: Annual sea level pressure, wind intensity, meridional and zonal wind, cloudiness, sea surface temperature. 


\section{Supplementary Figure 5}

\section{Number of climatic shifts in each geographical cell}

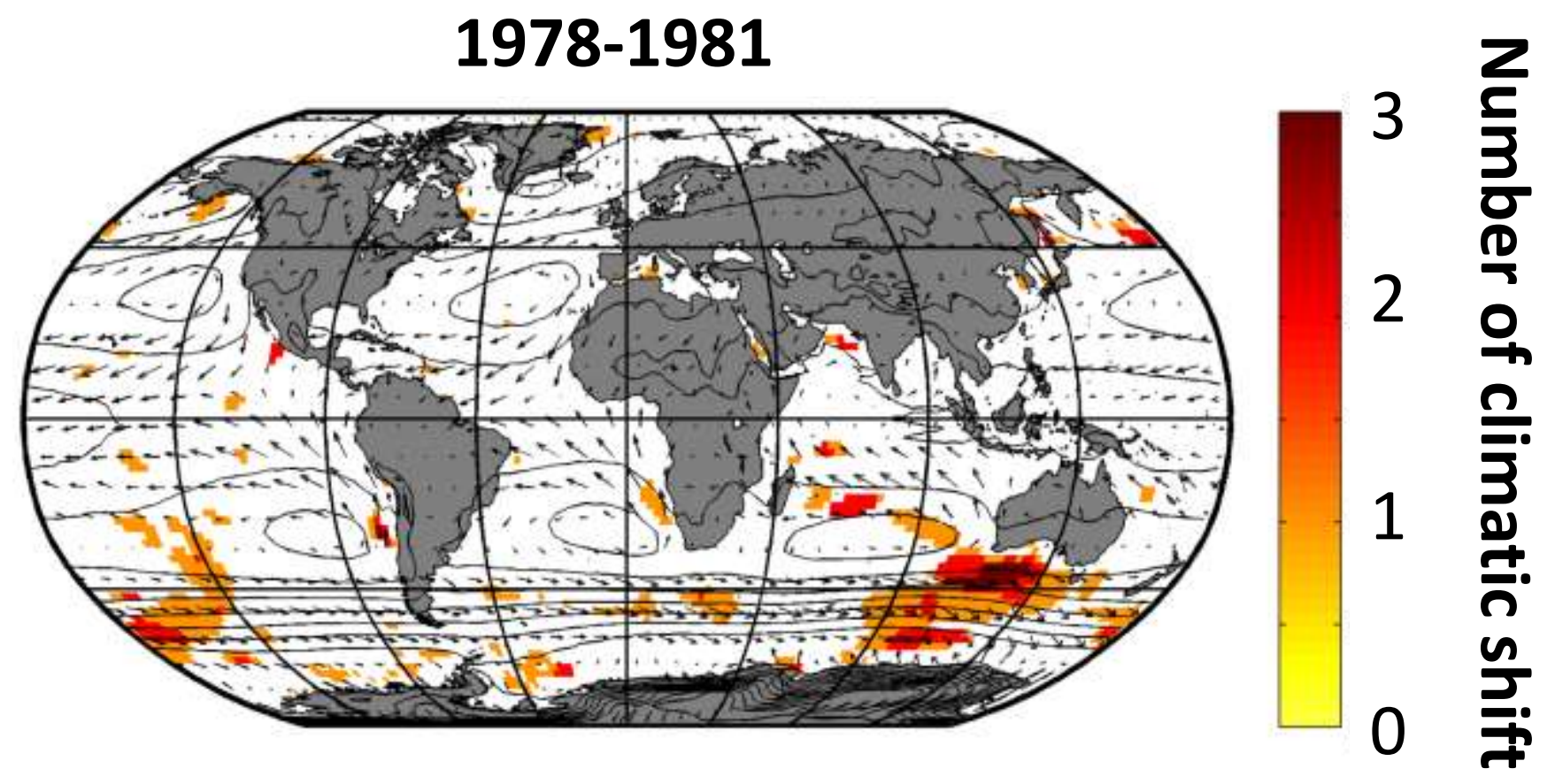

Black arrow: direction and strengh of mean annual wind (1960-2015) Red line: isobar based on annual SLP for the period 1960-2015 White area: no significant species' shift (threshold $>3$ )

Six climatic parameters were used: Annual sea level pressure, wind intensity, meridional and zonal wind, cloudiness, sea surface temperature. 


\section{Supplementary Figure 5}

\section{Number of climatic shifts in each geographical cell}

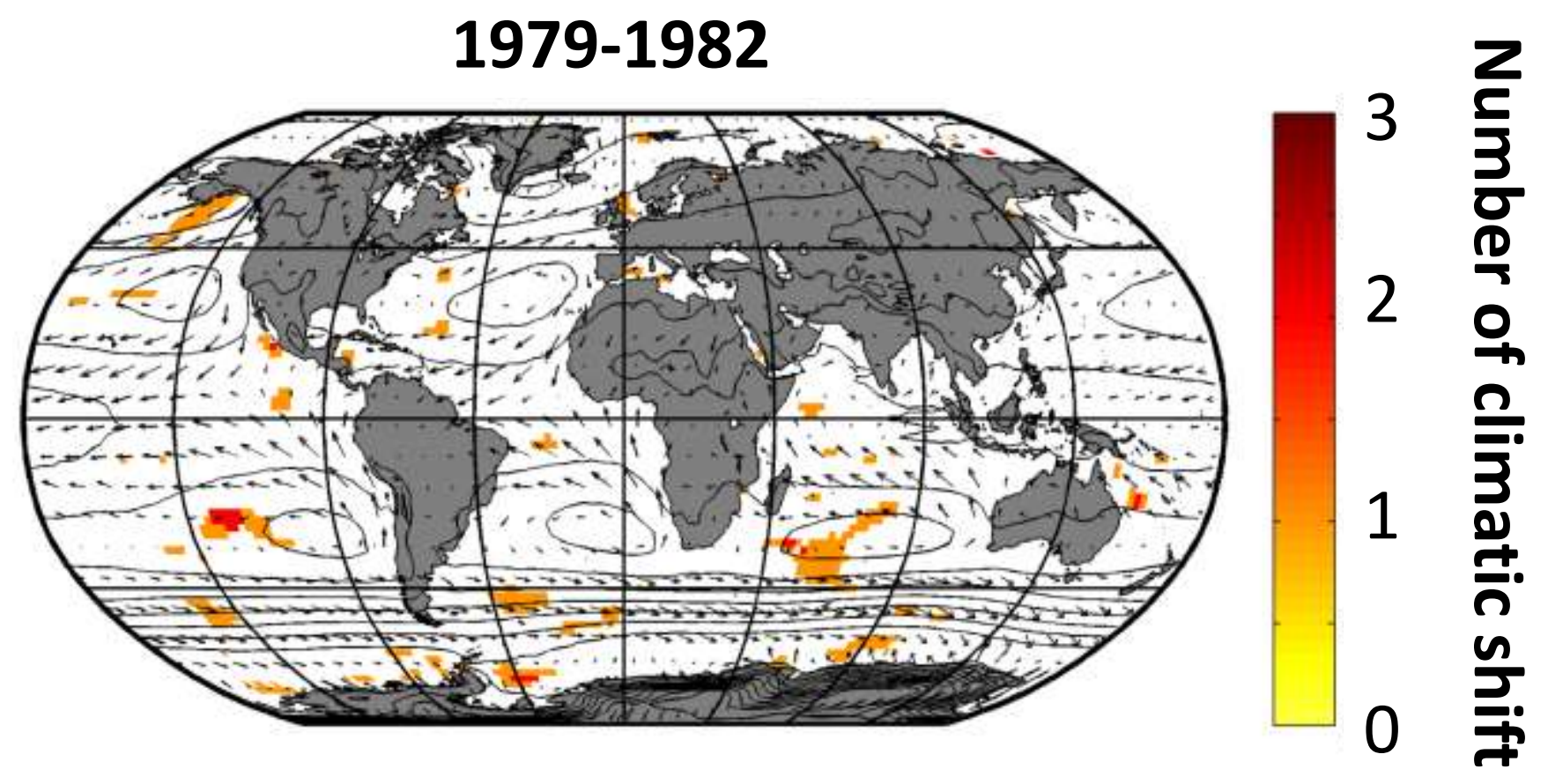

Black arrow: direction and strengh of mean annual wind (1960-2015) Red line: isobar based on annual SLP for the period 1960-2015 White area: no significant species' shift (threshold $>3$ )

Six climatic parameters were used: Annual sea level pressure, wind intensity, meridional and zonal wind, cloudiness, sea surface temperature. 


\section{Supplementary Figure 5}

\section{Number of climatic shifts in each geographical cell}

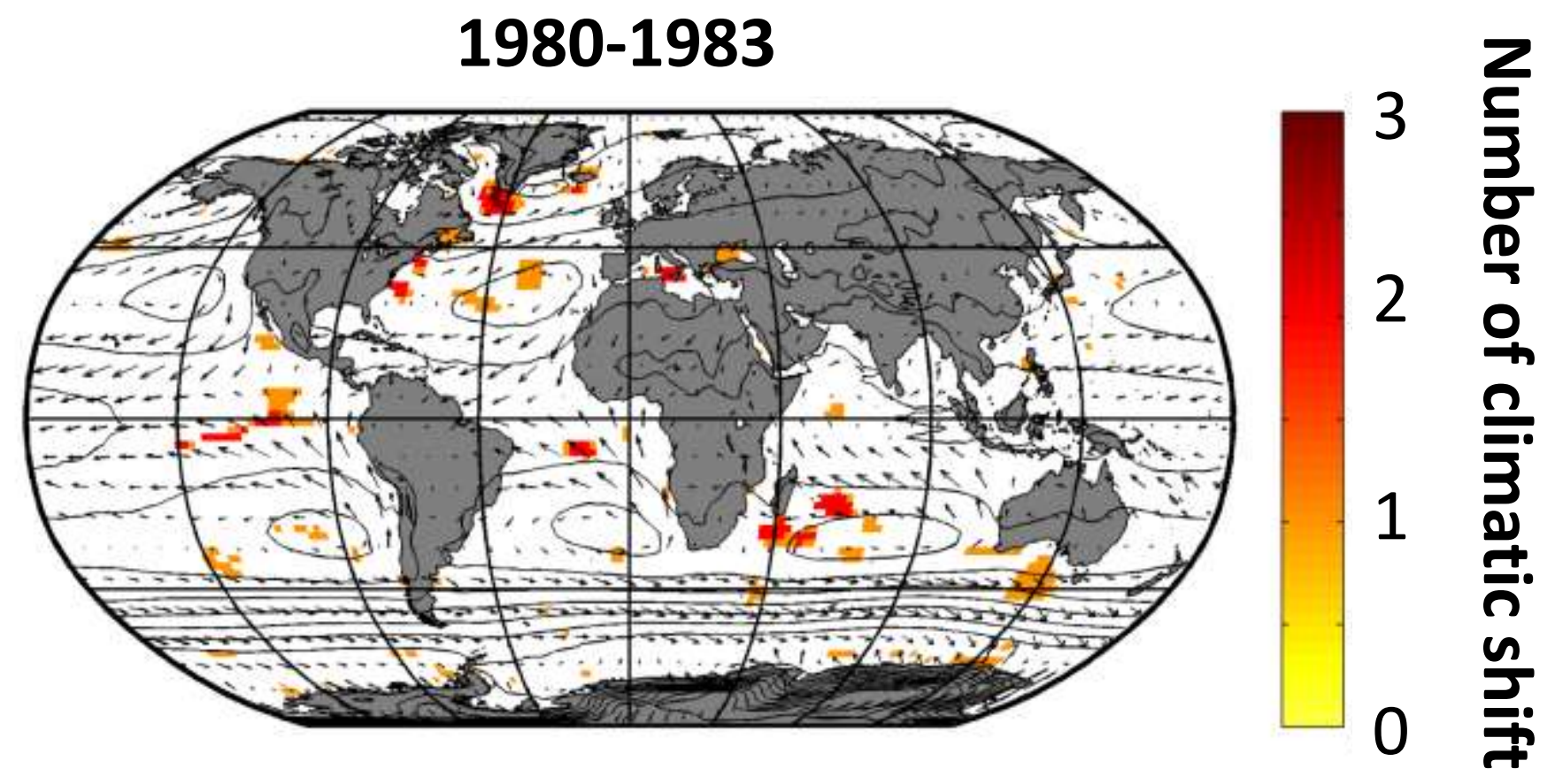

Black arrow: direction and strengh of mean annual wind (1960-2015) Red line: isobar based on annual SLP for the period 1960-2015 White area: no significant species' shift (threshold $>3$ )

Six climatic parameters were used: Annual sea level pressure, wind intensity, meridional and zonal wind, cloudiness, sea surface temperature. 


\section{Supplementary Figure 5}

\section{Number of climatic shifts in each geographical cell}

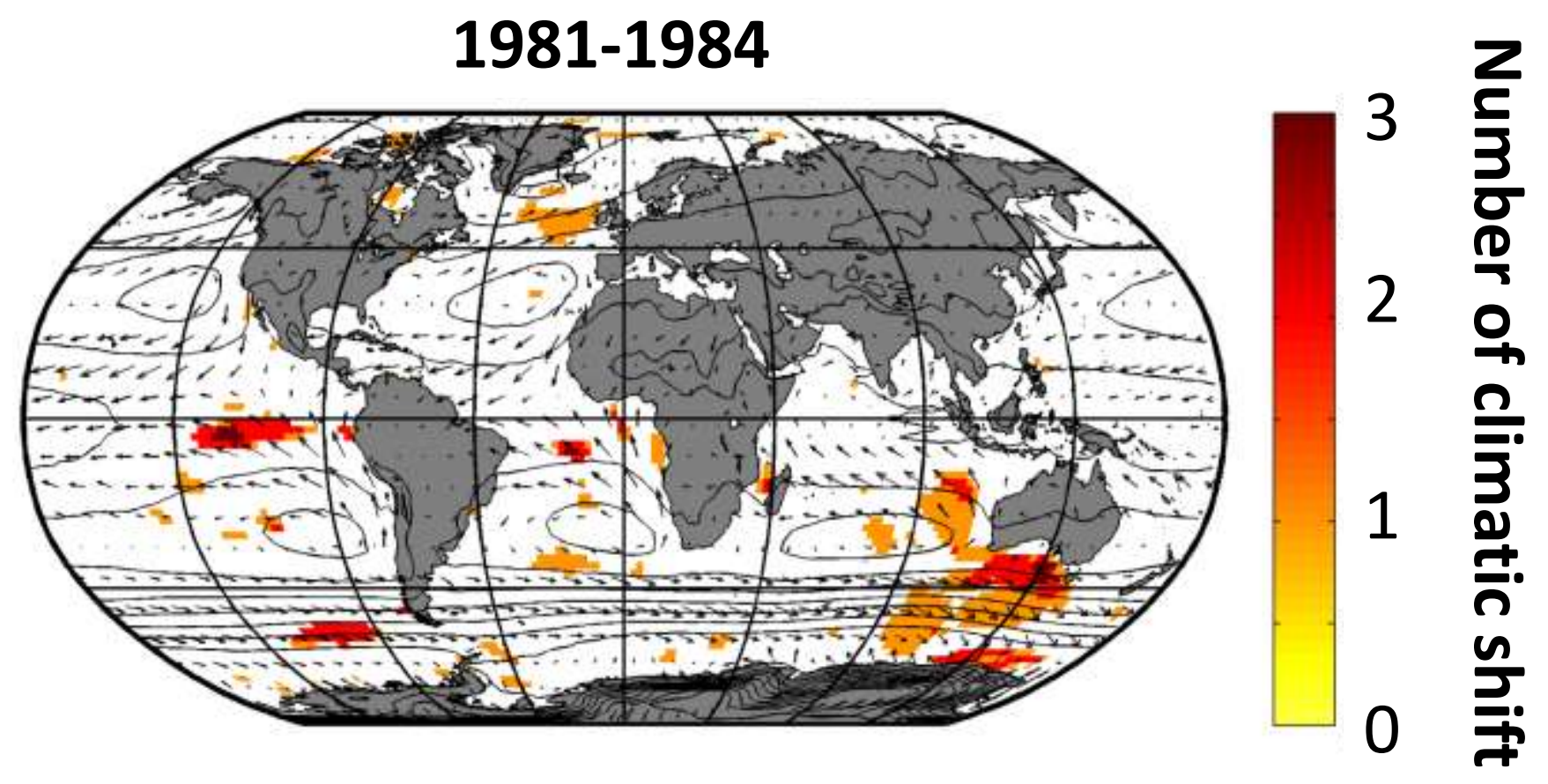

Black arrow: direction and strengh of mean annual wind (1960-2015) Red line: isobar based on annual SLP for the period 1960-2015 White area: no significant species' shift (threshold $>3$ )

Six climatic parameters were used: Annual sea level pressure, wind intensity, meridional and zonal wind, cloudiness, sea surface temperature. 


\section{Supplementary Figure 5}

\section{Number of climatic shifts in each geographical cell}

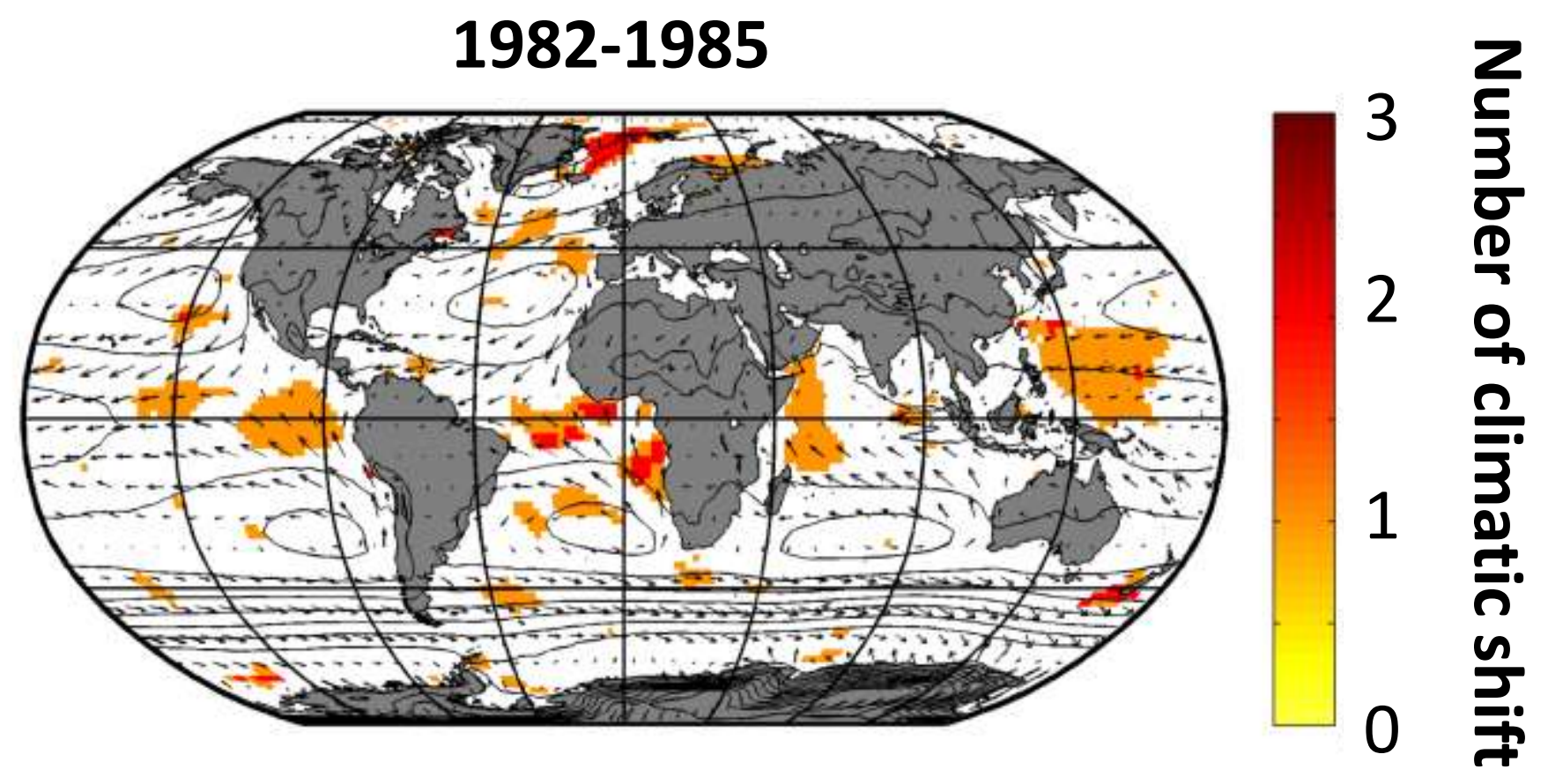

Black arrow: direction and strengh of mean annual wind (1960-2015) Red line: isobar based on annual SLP for the period 1960-2015 White area: no significant species' shift (threshold $>3$ )

Six climatic parameters were used: Annual sea level pressure, wind intensity, meridional and zonal wind, cloudiness, sea surface temperature. 


\section{Supplementary Figure 5}

\section{Number of climatic shifts in each geographical cell}

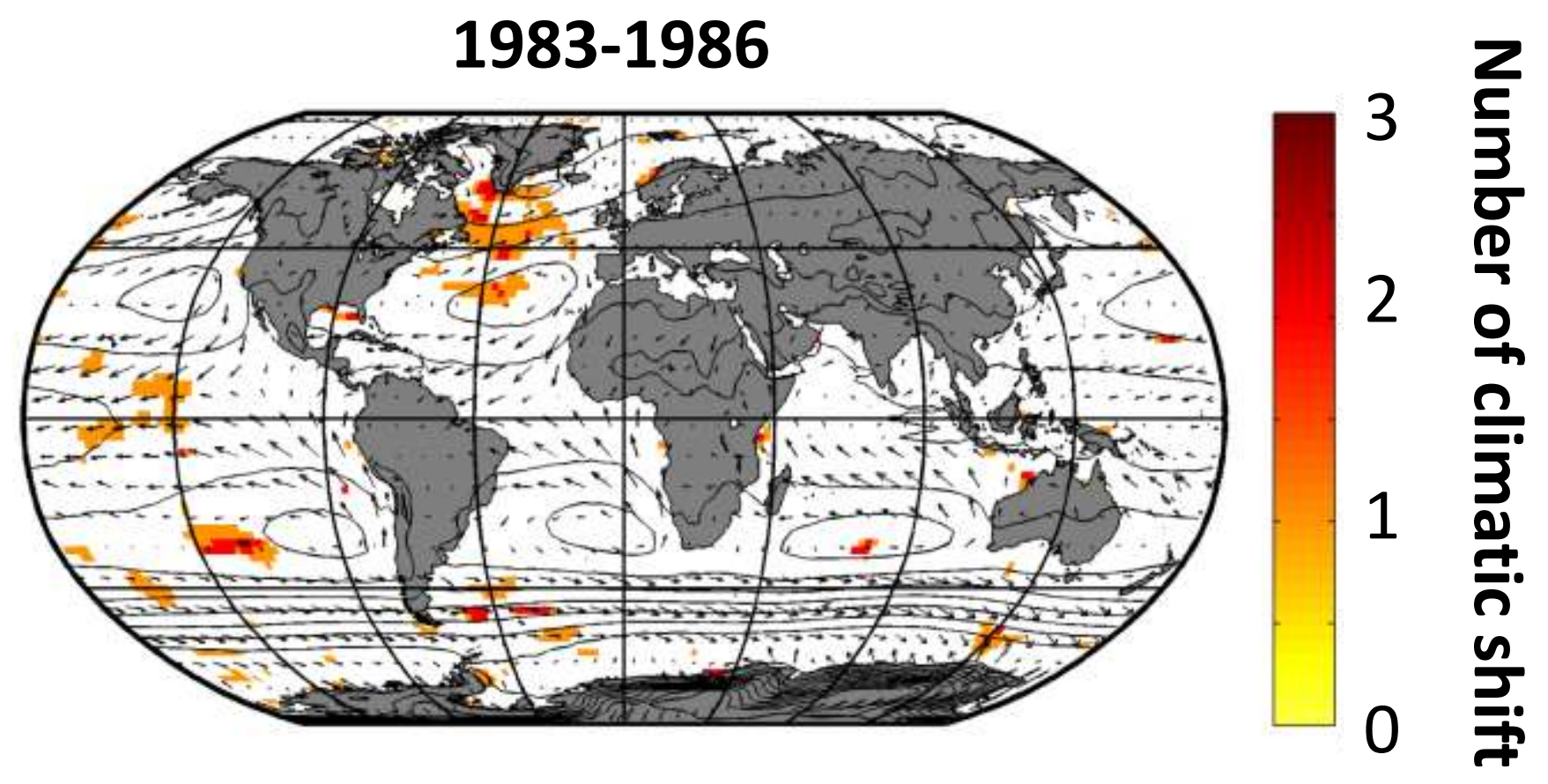

Black arrow: direction and strengh of mean annual wind (1960-2015) Red line: isobar based on annual SLP for the period 1960-2015 White area: no significant species' shift (threshold $>3$ )

Six climatic parameters were used: Annual sea level pressure, wind intensity, meridional and zonal wind, cloudiness, sea surface temperature. 


\section{Supplementary Figure 5}

\section{Number of climatic shifts in each geographical cell}

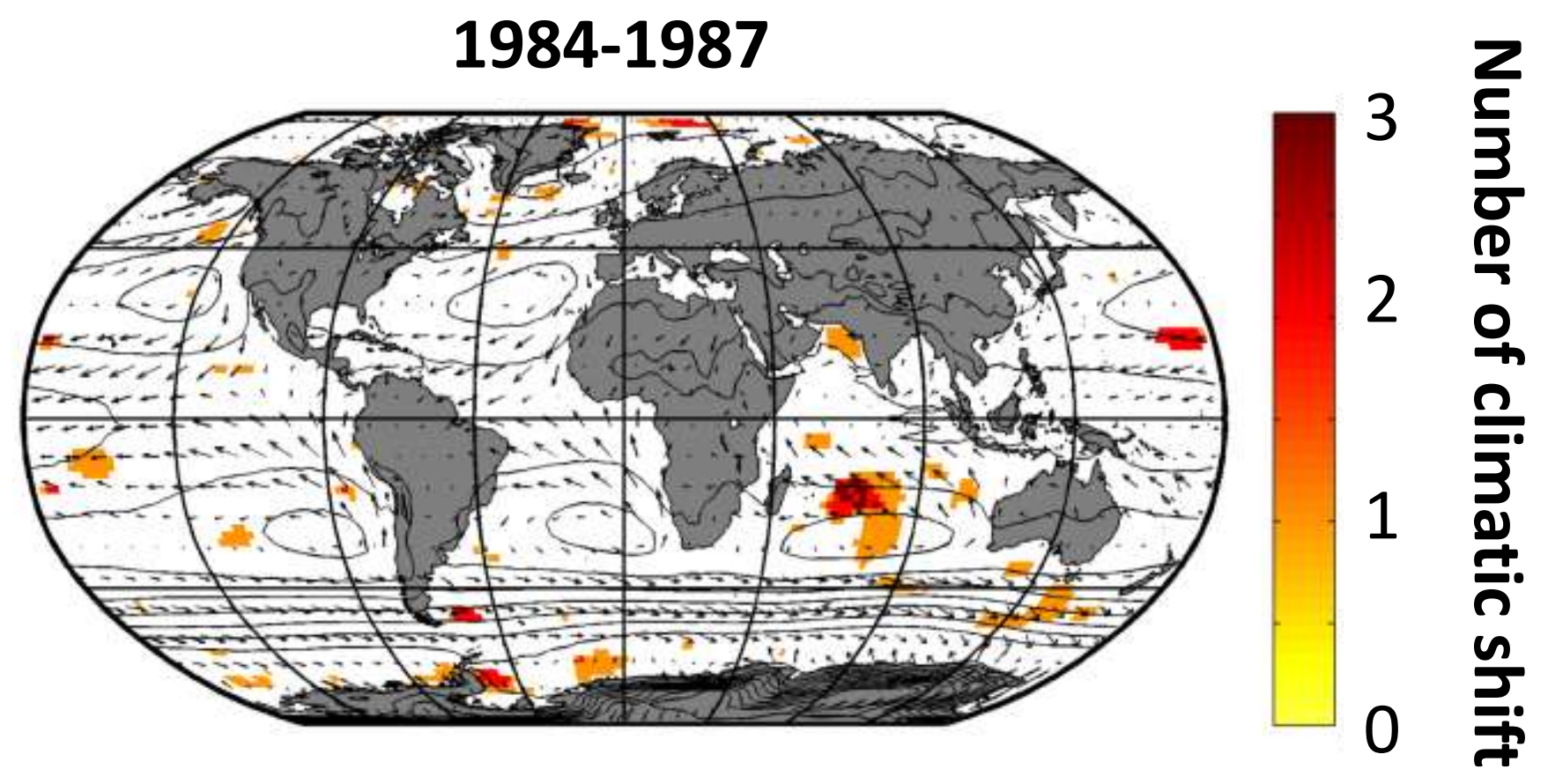

Black arrow: direction and strengh of mean annual wind (1960-2015) Red line: isobar based on annual SLP for the period 1960-2015 White area: no significant species' shift (threshold $>3$ )

Six climatic parameters were used: Annual sea level pressure, wind intensity, meridional and zonal wind, cloudiness, sea surface temperature. 


\section{Supplementary Figure 5}

\section{Number of climatic shifts in each geographical cell}

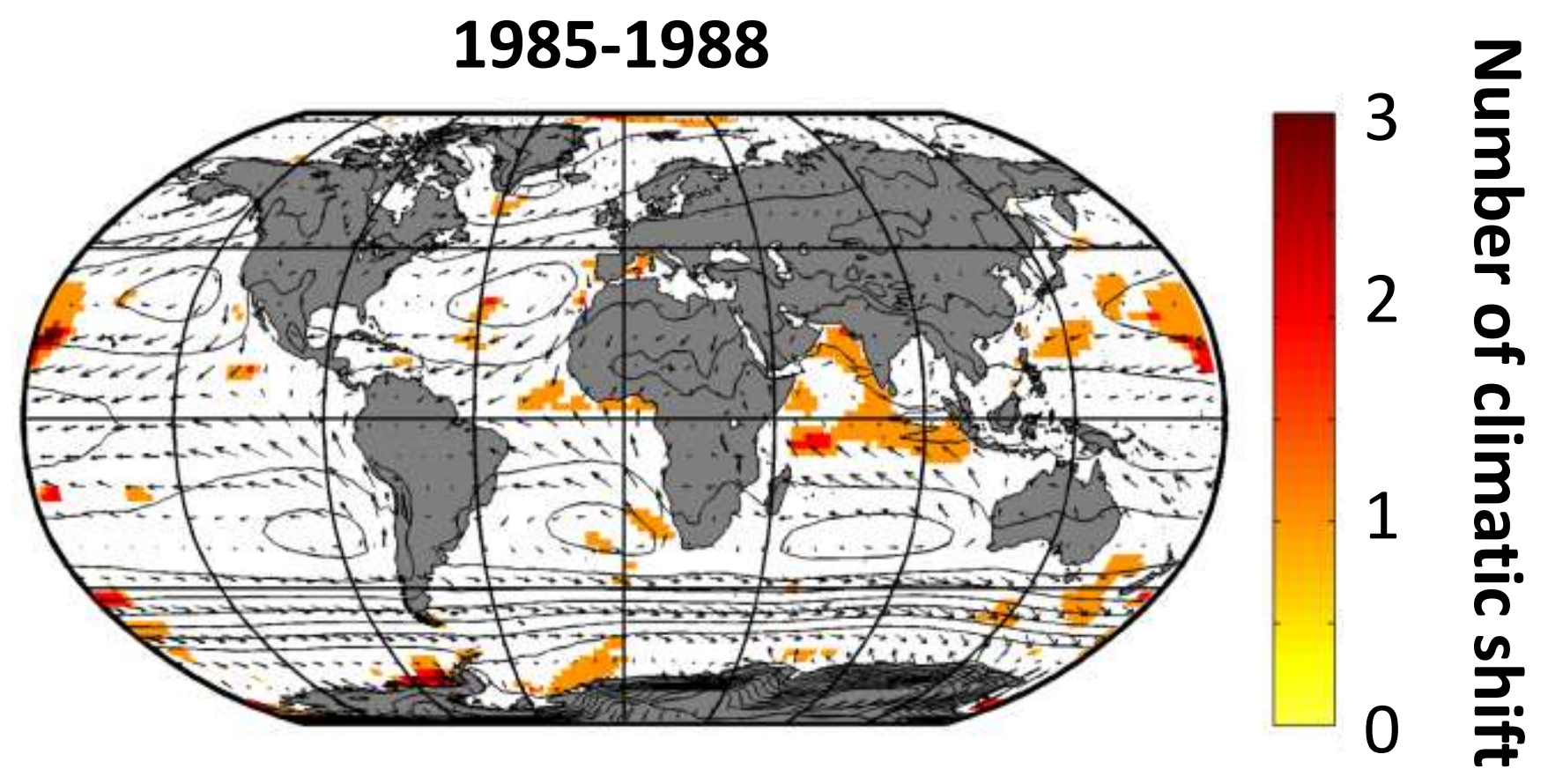

Black arrow: direction and strengh of mean annual wind (1960-2015) Red line: isobar based on annual SLP for the period 1960-2015 White area: no significant species' shift (threshold $>3$ )

Six climatic parameters were used: Annual sea level pressure, wind intensity, meridional and zonal wind, cloudiness, sea surface temperature. 


\section{Supplementary Figure 5}

\section{Number of climatic shifts in each geographical cell}

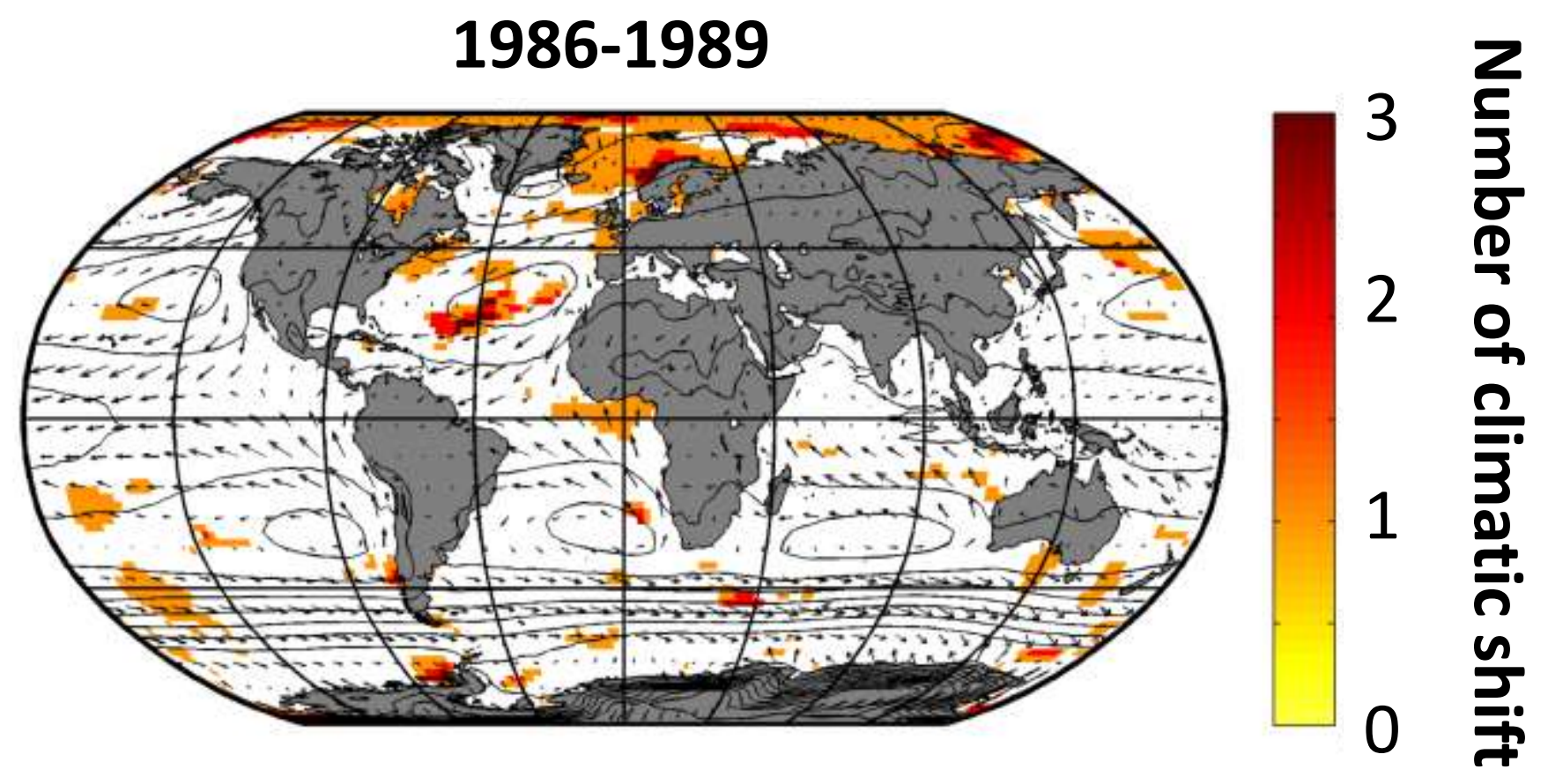

Black arrow: direction and strengh of mean annual wind (1960-2015) Red line: isobar based on annual SLP for the period 1960-2015 White area: no significant species' shift (threshold $>3$ )

Six climatic parameters were used: Annual sea level pressure, wind intensity, meridional and zonal wind, cloudiness, sea surface temperature. 


\section{Supplementary Figure 5}

\section{Number of climatic shifts in each geographical cell}

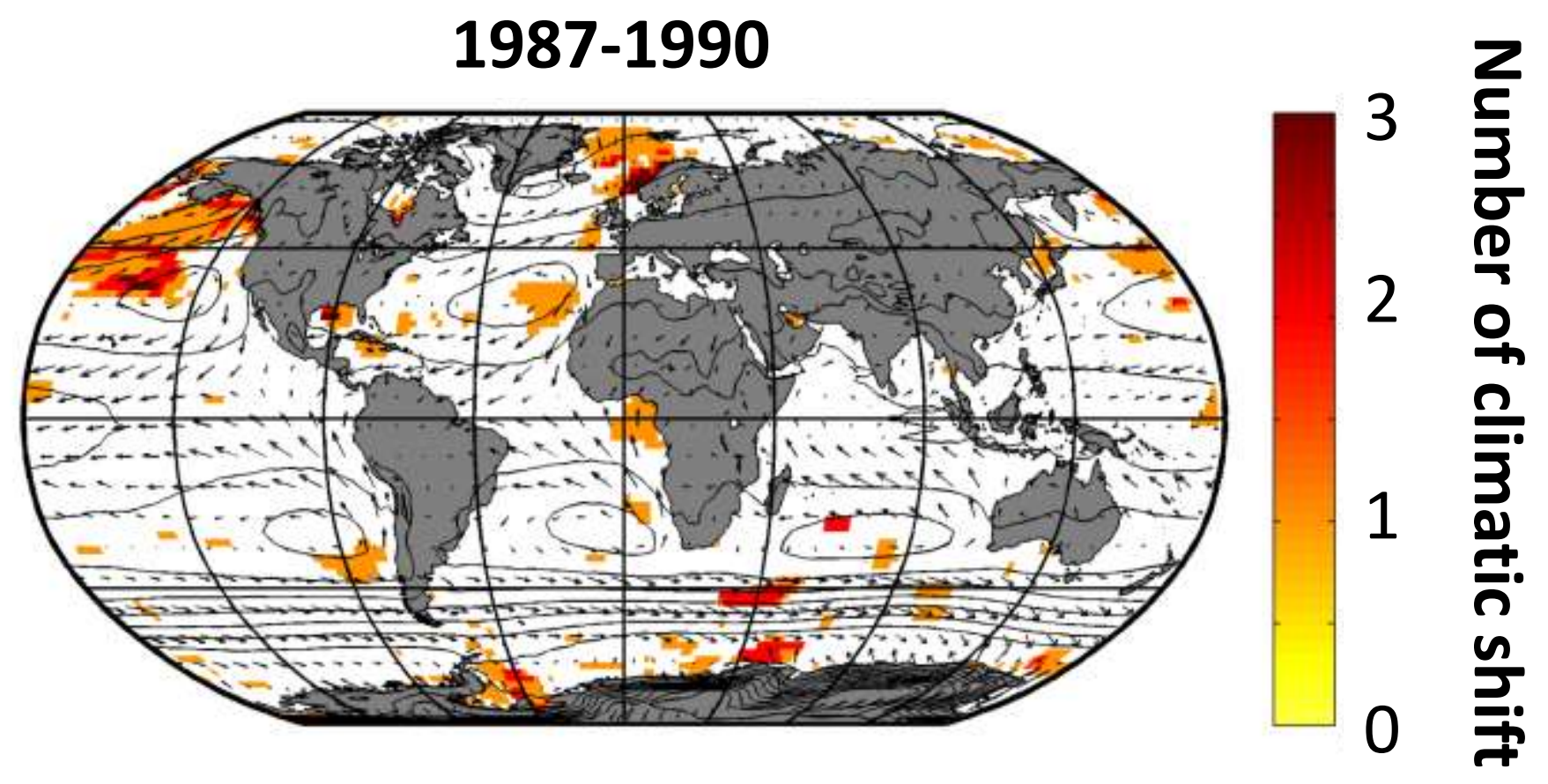

Black arrow: direction and strengh of mean annual wind (1960-2015) Red line: isobar based on annual SLP for the period 1960-2015 White area: no significant species' shift (threshold $>3$ )

Six climatic parameters were used: Annual sea level pressure, wind intensity, meridional and zonal wind, cloudiness, sea surface temperature. 


\section{Supplementary Figure 5}

\section{Number of climatic shifts in each geographical cell}

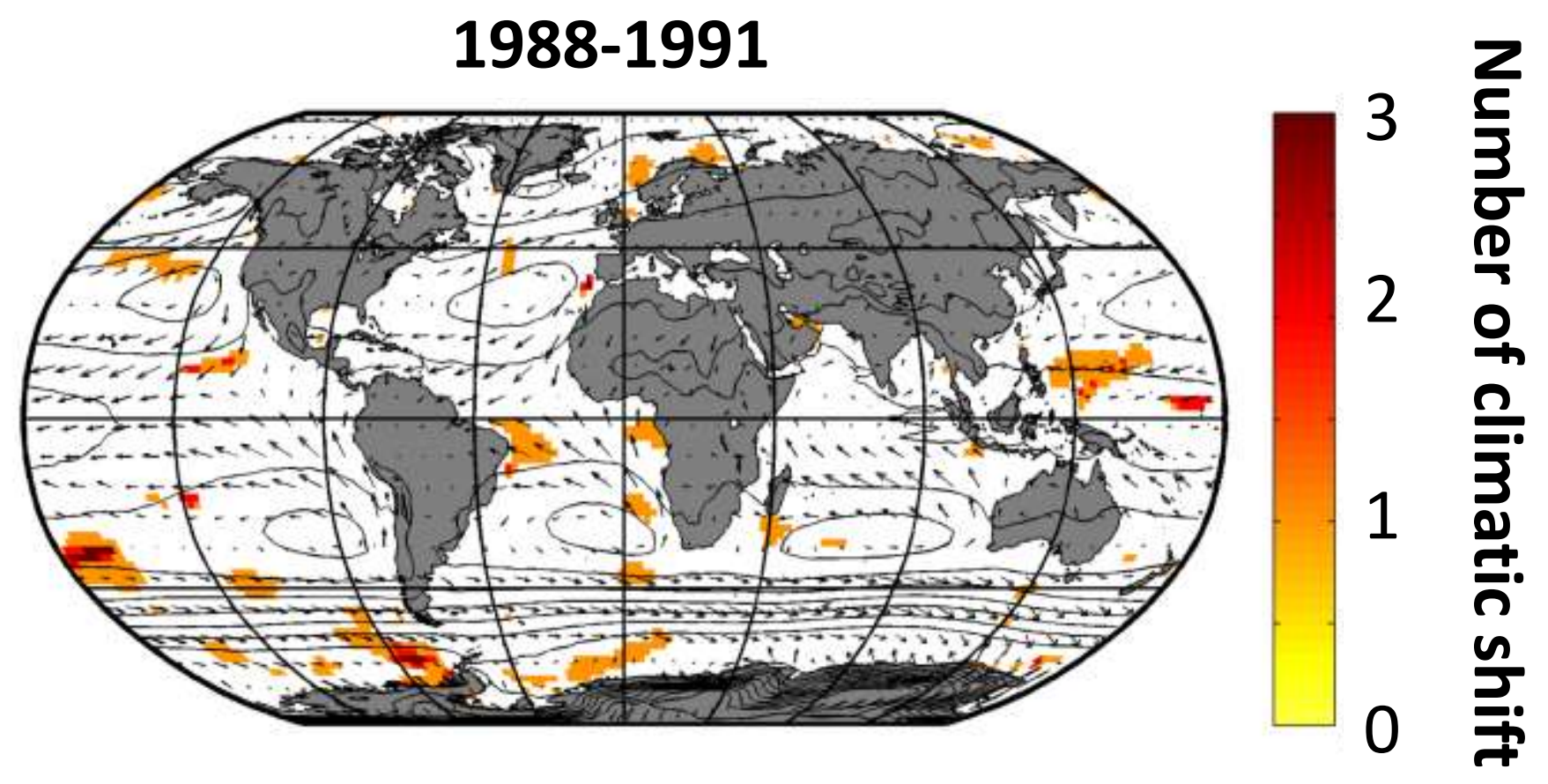

Black arrow: direction and strengh of mean annual wind (1960-2015) Red line: isobar based on annual SLP for the period 1960-2015 White area: no significant species' shift (threshold $>3$ )

Six climatic parameters were used: Annual sea level pressure, wind intensity, meridional and zonal wind, cloudiness, sea surface temperature. 


\section{Supplementary Figure 5}

\section{Number of climatic shifts in each geographical cell}

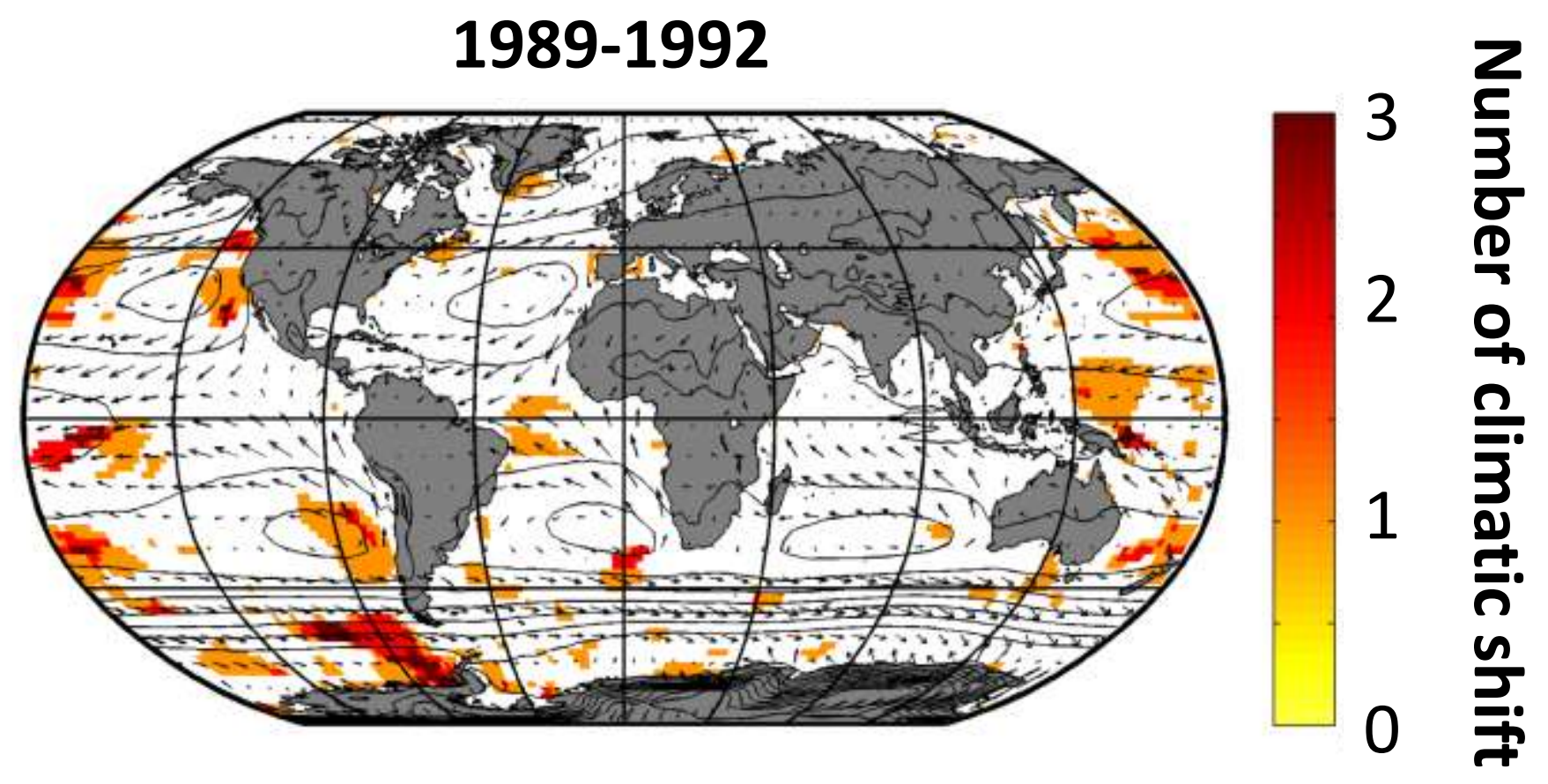

Black arrow: direction and strengh of mean annual wind (1960-2015) Red line: isobar based on annual SLP for the period 1960-2015 White area: no significant species' shift (threshold $>3$ )

Six climatic parameters were used: Annual sea level pressure, wind intensity, meridional and zonal wind, cloudiness, sea surface temperature. 


\section{Supplementary Figure 5}

\section{Number of climatic shifts in each geographical cell}

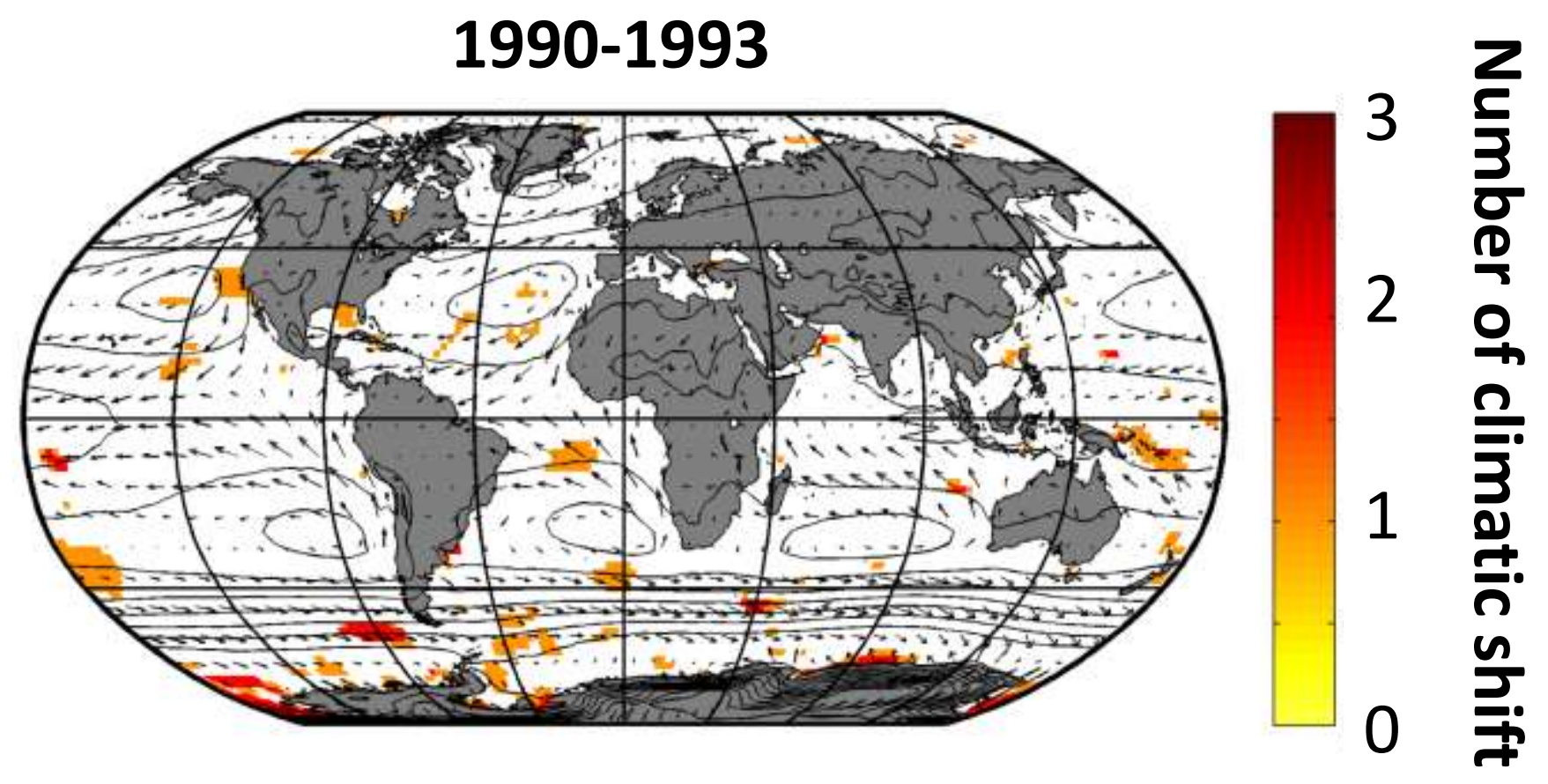

Black arrow: direction and strengh of mean annual wind (1960-2015) Red line: isobar based on annual SLP for the period 1960-2015 White area: no significant species' shift (threshold $>3$ )

Six climatic parameters were used: Annual sea level pressure, wind intensity, meridional and zonal wind, cloudiness, sea surface temperature. 


\section{Supplementary Figure 5}

\section{Number of climatic shifts in each geographical cell}

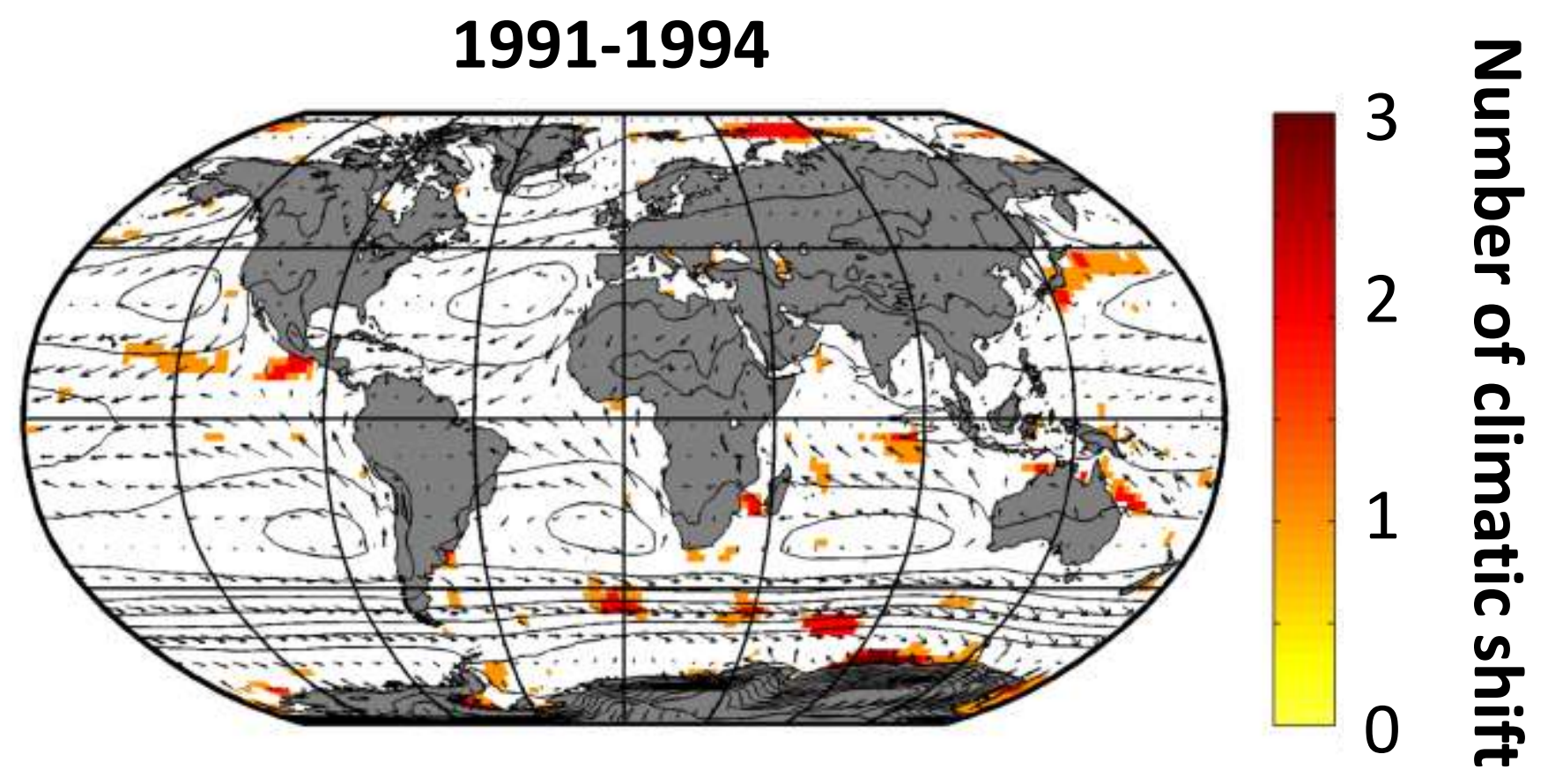

Black arrow: direction and strengh of mean annual wind (1960-2015) Red line: isobar based on annual SLP for the period 1960-2015 White area: no significant species' shift (threshold $>3$ )

Six climatic parameters were used: Annual sea level pressure, wind intensity, meridional and zonal wind, cloudiness, sea surface temperature. 


\section{Supplementary Figure 5}

\section{Number of climatic shifts in each geographical cell}

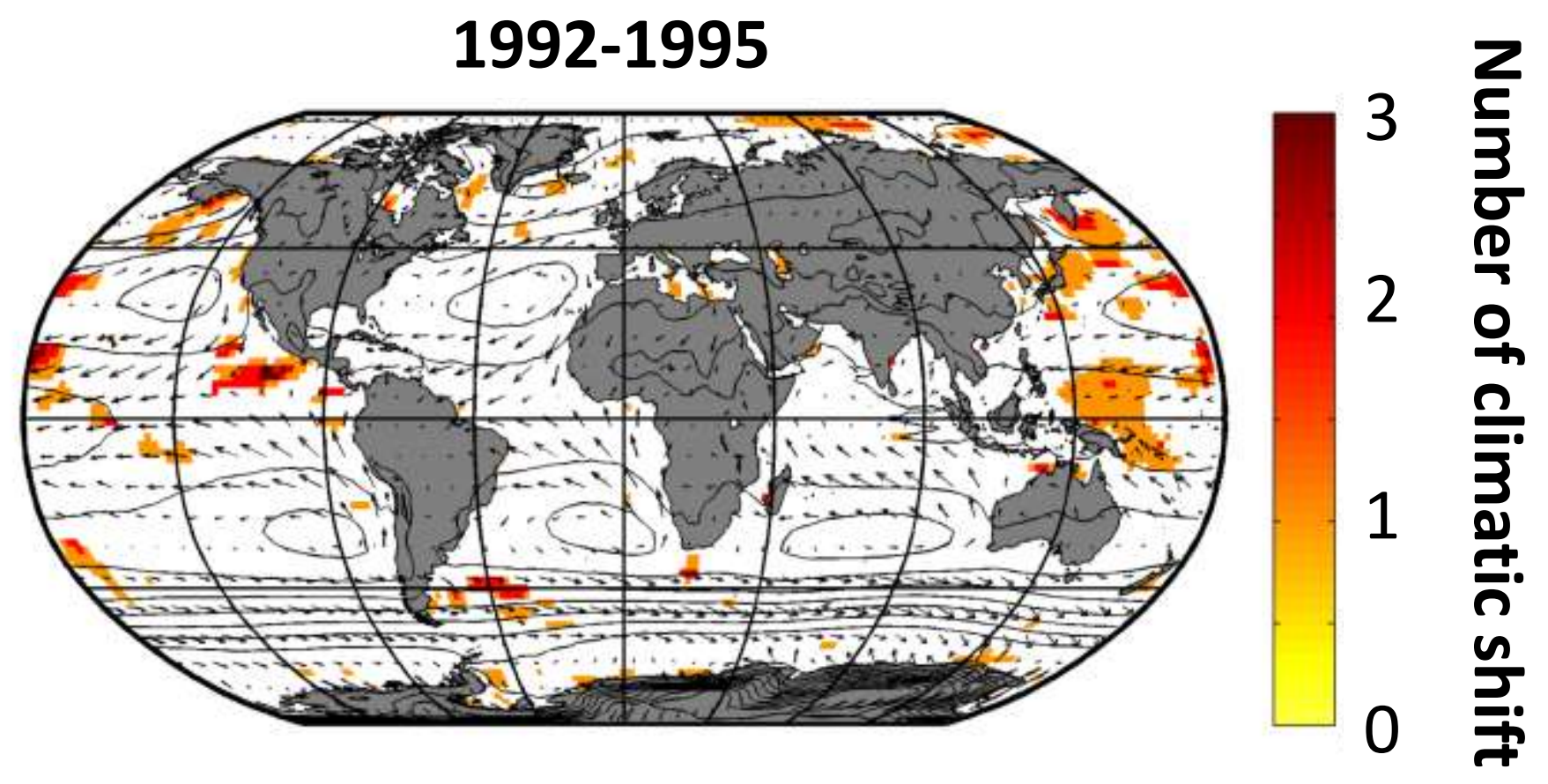

Black arrow: direction and strengh of mean annual wind (1960-2015) Red line: isobar based on annual SLP for the period 1960-2015 White area: no significant species' shift (threshold $>3$ )

Six climatic parameters were used: Annual sea level pressure, wind intensity, meridional and zonal wind, cloudiness, sea surface temperature. 


\section{Supplementary Figure 5}

\section{Number of climatic shifts in each geographical cell}

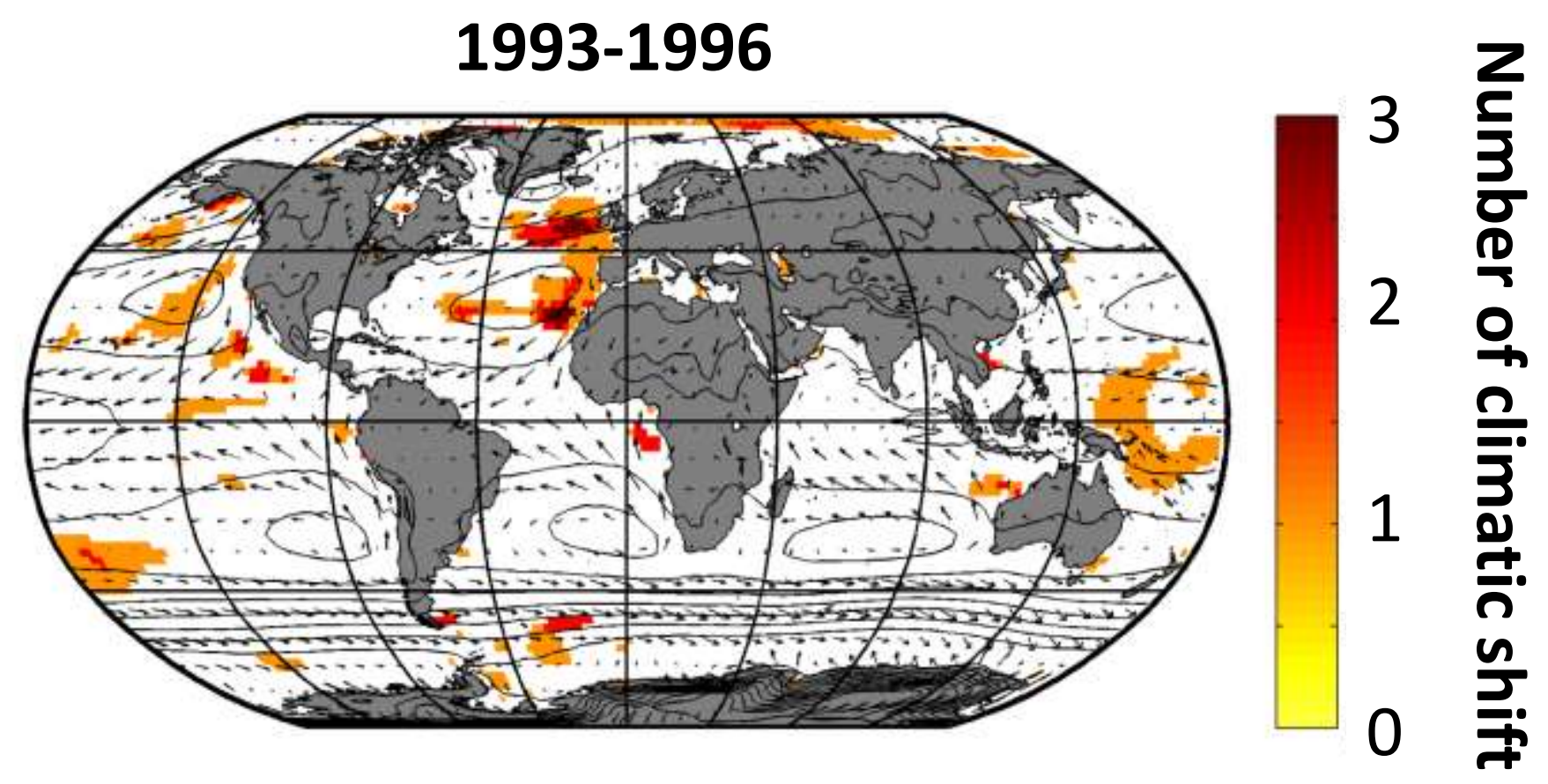

Black arrow: direction and strengh of mean annual wind (1960-2015) Red line: isobar based on annual SLP for the period 1960-2015 White area: no significant species' shift (threshold $>3$ )

Six climatic parameters were used: Annual sea level pressure, wind intensity, meridional and zonal wind, cloudiness, sea surface temperature. 


\section{Supplementary Figure 5}

\section{Number of climatic shifts in each geographical cell}

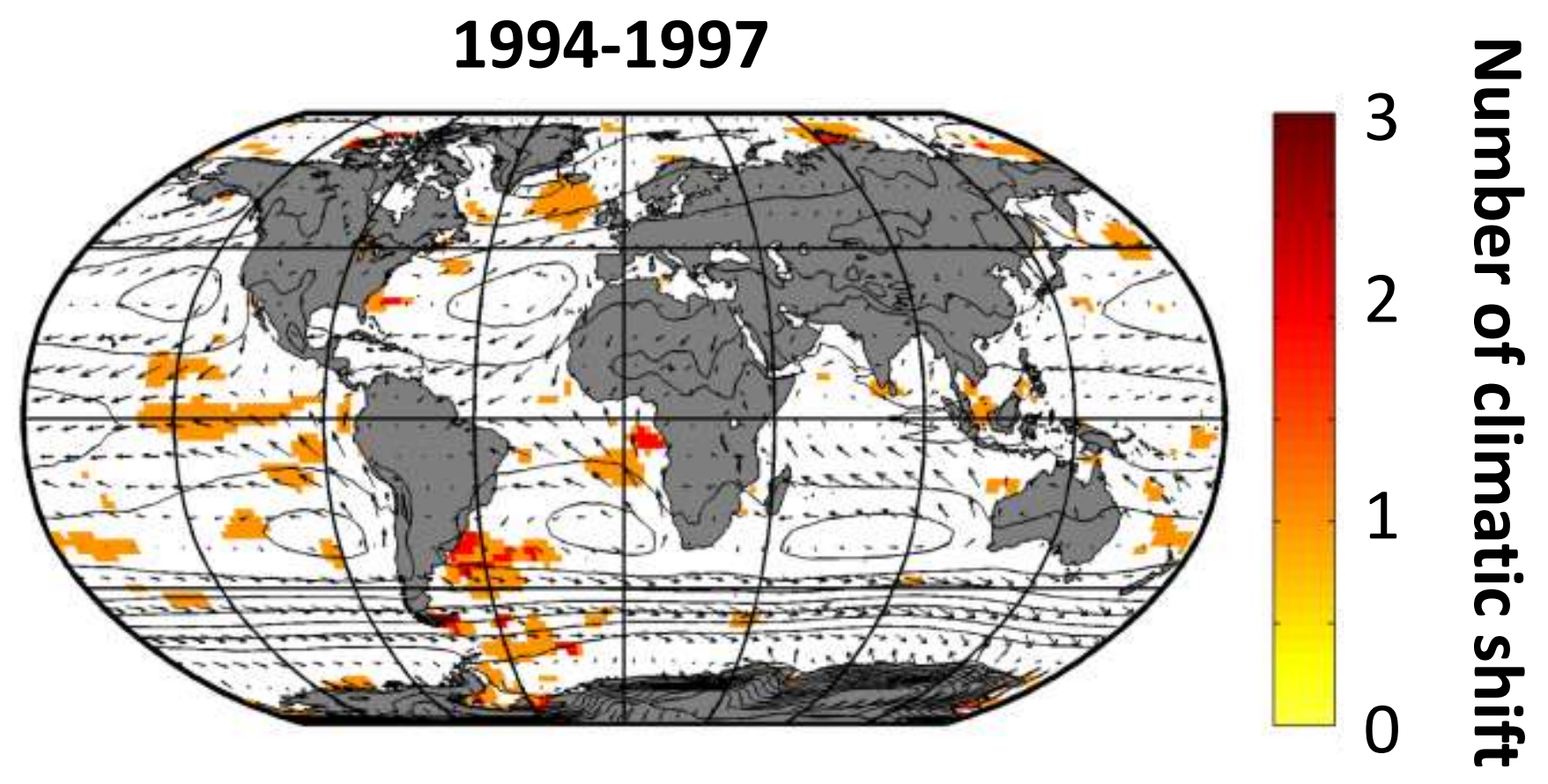

Black arrow: direction and strengh of mean annual wind (1960-2015) Red line: isobar based on annual SLP for the period 1960-2015 White area: no significant species' shift (threshold $>3$ )

Six climatic parameters were used: Annual sea level pressure, wind intensity, meridional and zonal wind, cloudiness, sea surface temperature. 


\section{Supplementary Figure 5}

\section{Number of climatic shifts in each geographical cell}

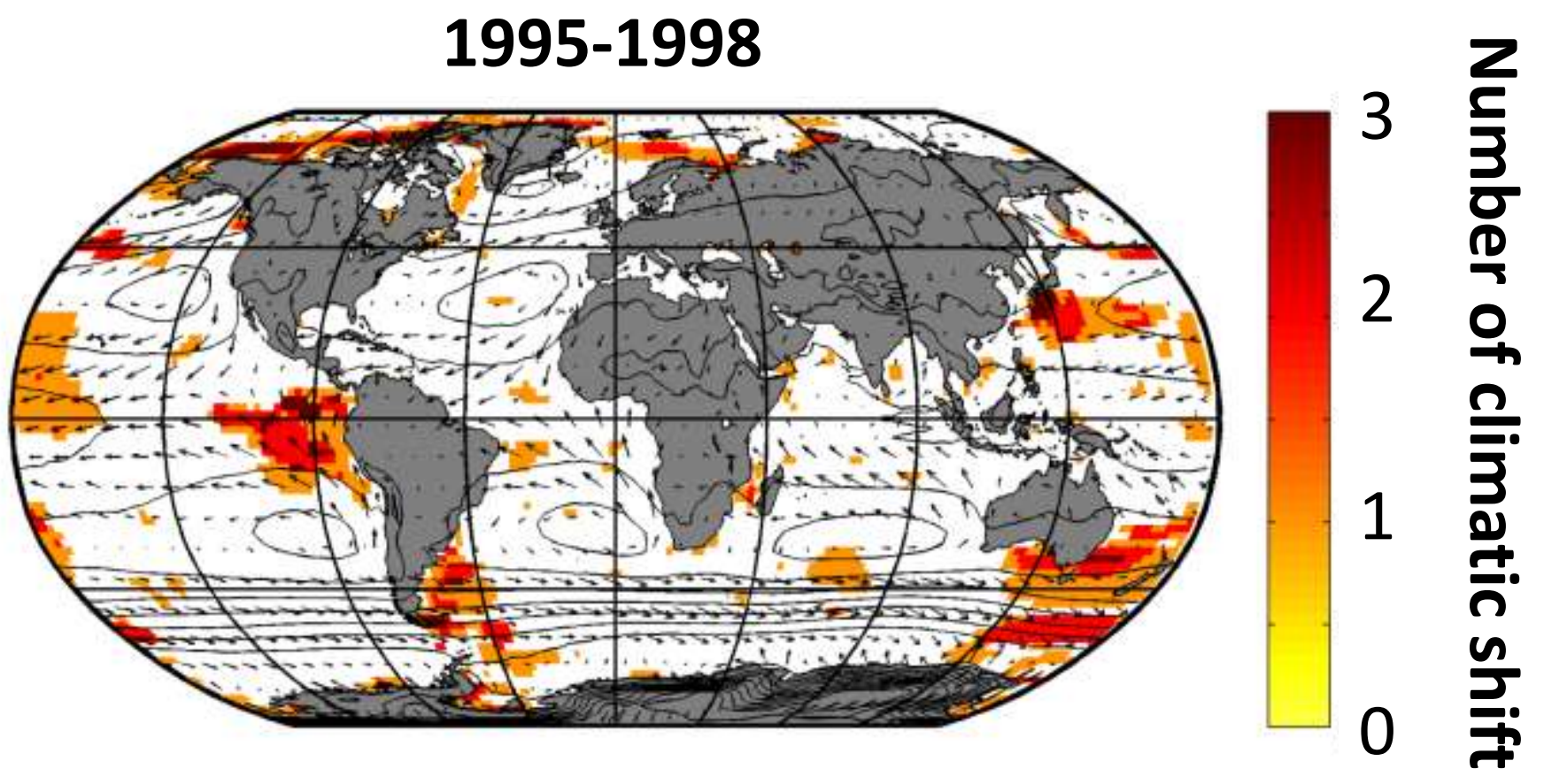

Black arrow: direction and strengh of mean annual wind (1960-2015) Red line: isobar based on annual SLP for the period 1960-2015 White area: no significant species' shift (threshold $>3$ )

Six climatic parameters were used: Annual sea level pressure, wind intensity, meridional and zonal wind, cloudiness, sea surface temperature. 


\section{Supplementary Figure 5}

\section{Number of climatic shifts in each geographical cell}

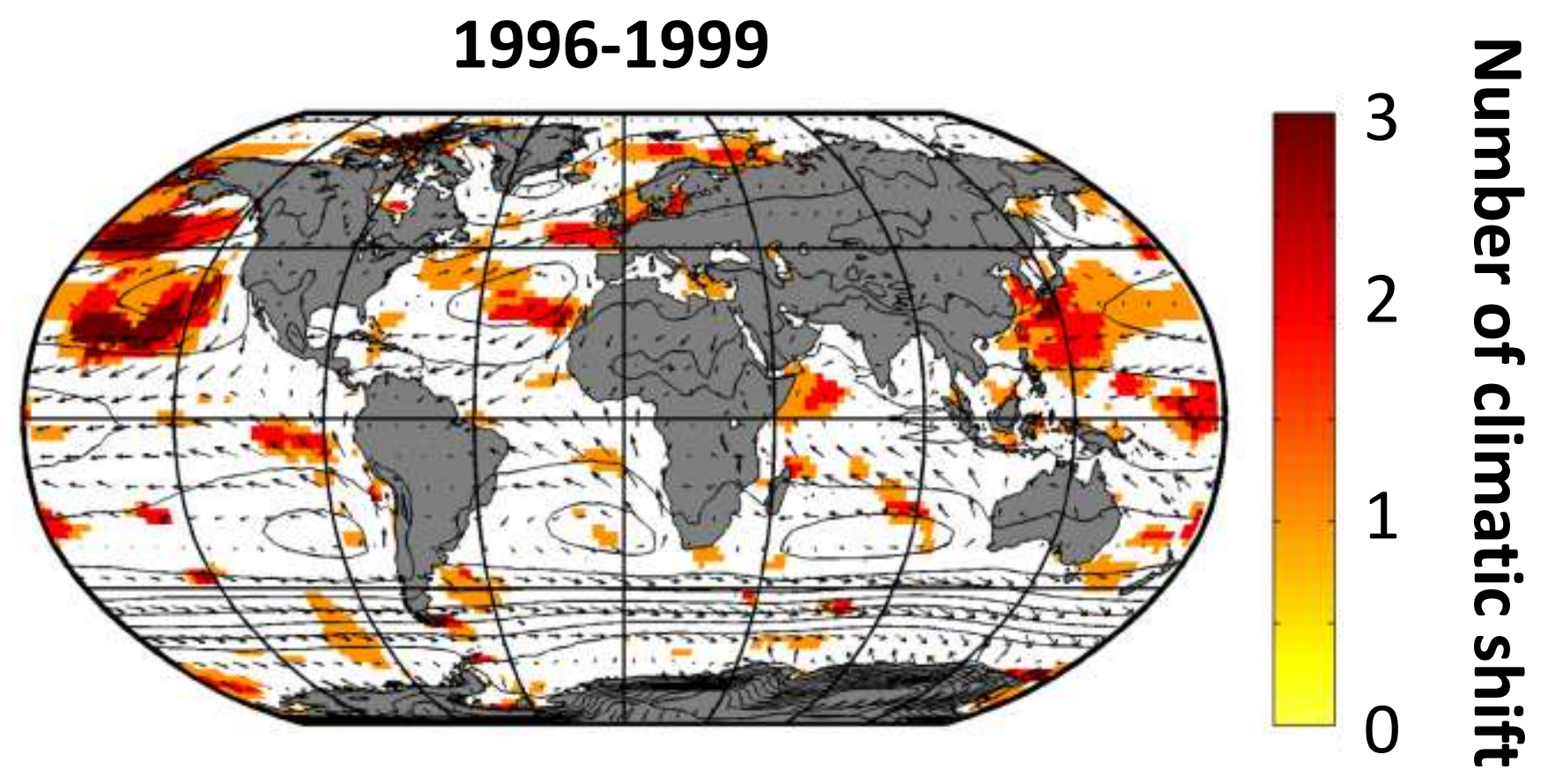

Black arrow: direction and strengh of mean annual wind (1960-2015) Red line: isobar based on annual SLP for the period 1960-2015 White area: no significant species' shift (threshold $>3$ )

Six climatic parameters were used: Annual sea level pressure, wind intensity, meridional and zonal wind, cloudiness, sea surface temperature. 


\section{Supplementary Figure 5}

\section{Number of climatic shifts in each geographical cell}

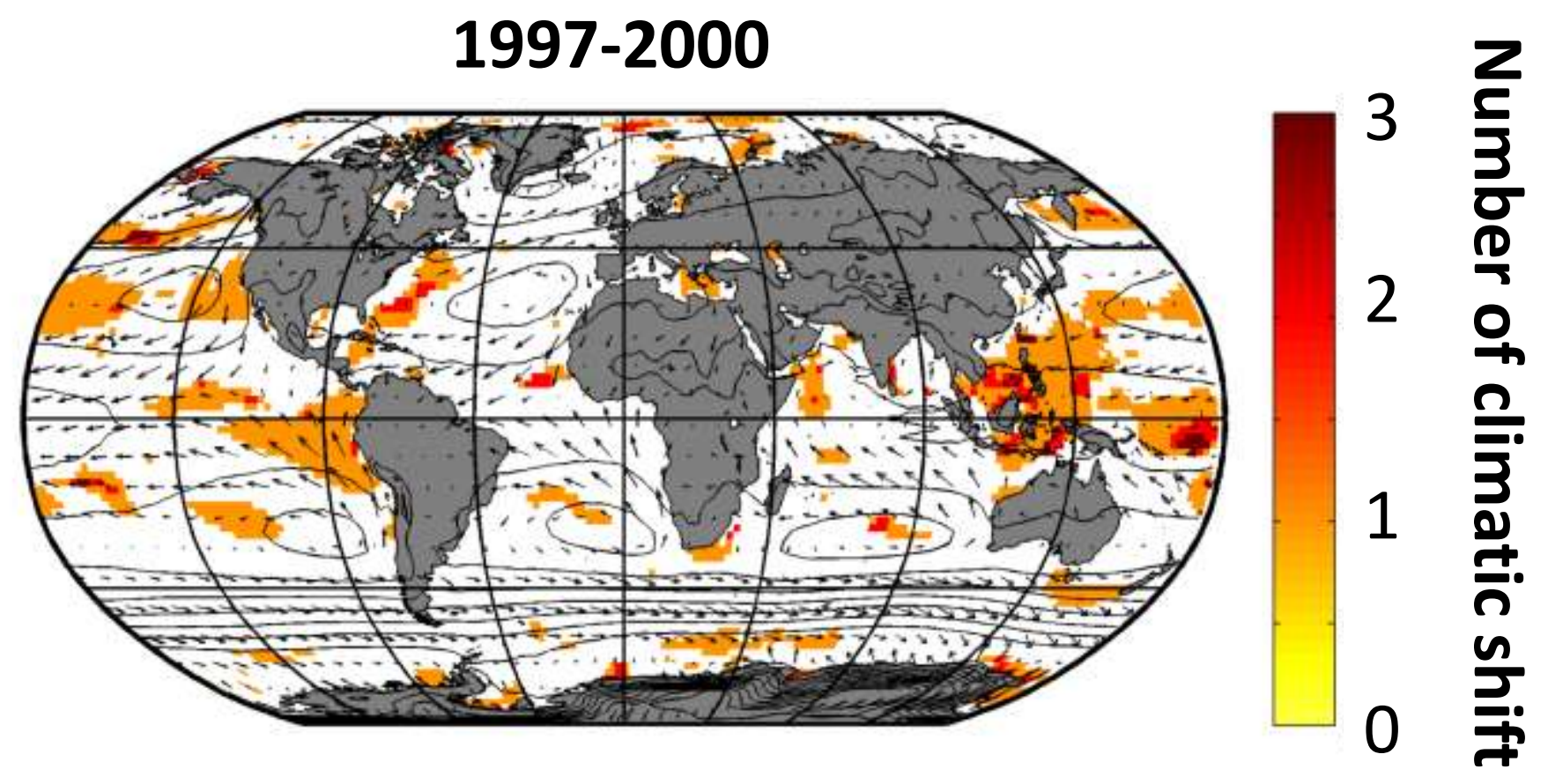

Black arrow: direction and strengh of mean annual wind (1960-2015) Red line: isobar based on annual SLP for the period 1960-2015 White area: no significant species' shift (threshold $>3$ )

Six climatic parameters were used: Annual sea level pressure, wind intensity, meridional and zonal wind, cloudiness, sea surface temperature. 


\section{Supplementary Figure 5}

\section{Number of climatic shifts in each geographical cell}

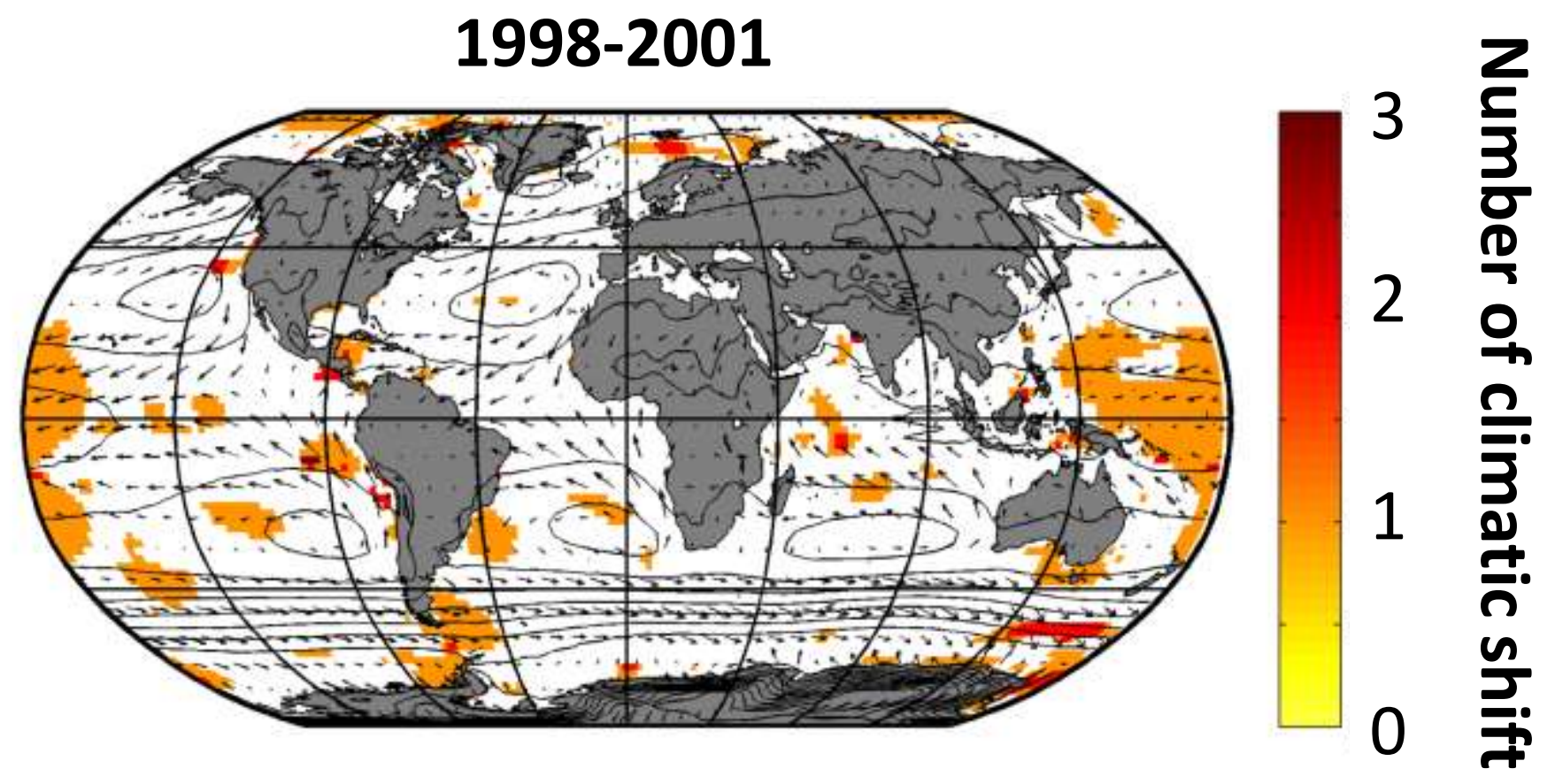

Black arrow: direction and strengh of mean annual wind (1960-2015) Red line: isobar based on annual SLP for the period 1960-2015 White area: no significant species' shift (threshold $>3$ )

Six climatic parameters were used: Annual sea level pressure, wind intensity, meridional and zonal wind, cloudiness, sea surface temperature. 


\section{Supplementary Figure 5}

\section{Number of climatic shifts in each geographical cell}

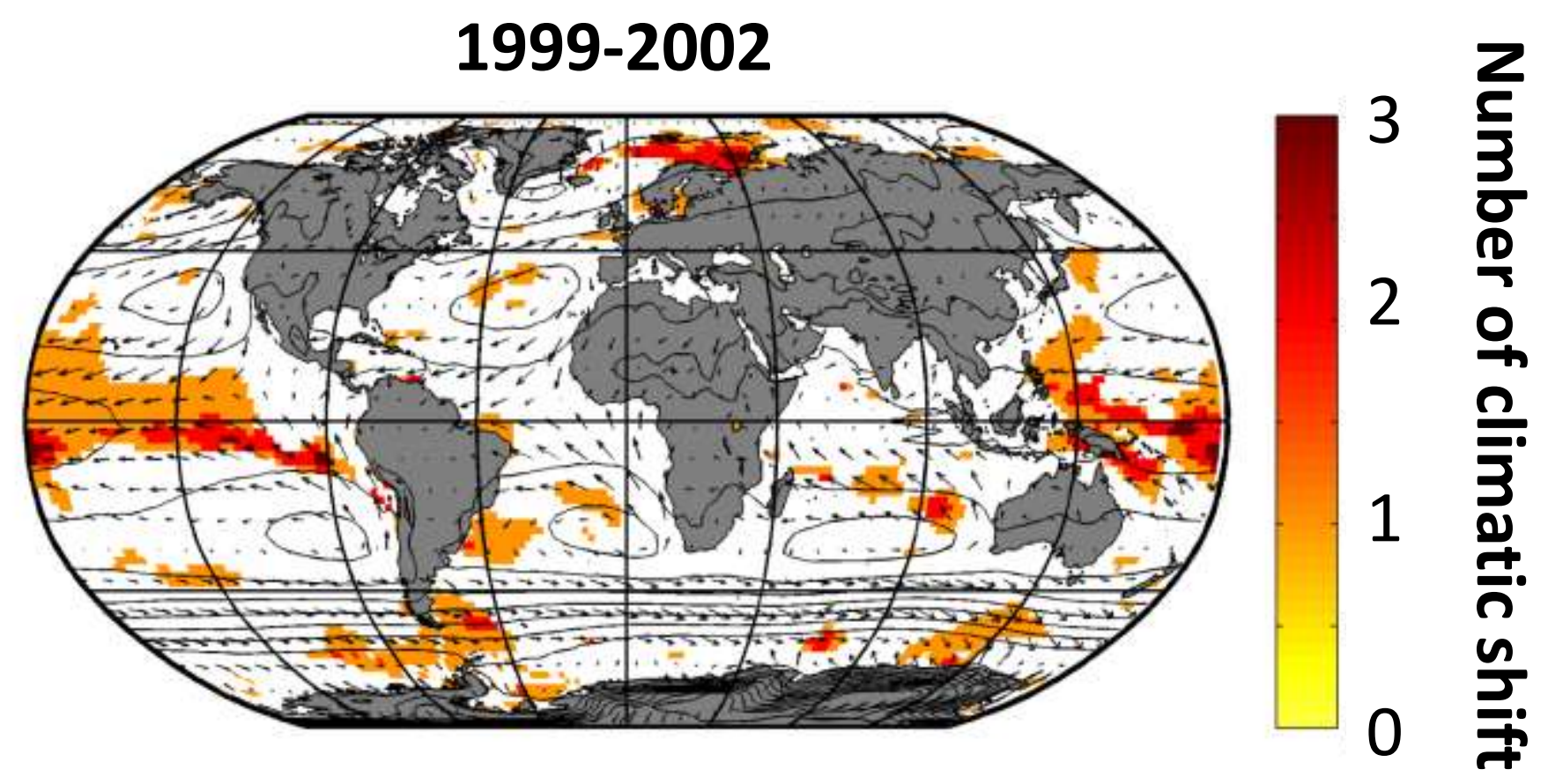

Black arrow: direction and strengh of mean annual wind (1960-2015) Red line: isobar based on annual SLP for the period 1960-2015 White area: no significant species' shift (threshold $>3$ )

Six climatic parameters were used: Annual sea level pressure, wind intensity, meridional and zonal wind, cloudiness, sea surface temperature. 


\section{Supplementary Figure 5}

\section{Number of climatic shifts in each geographical cell}

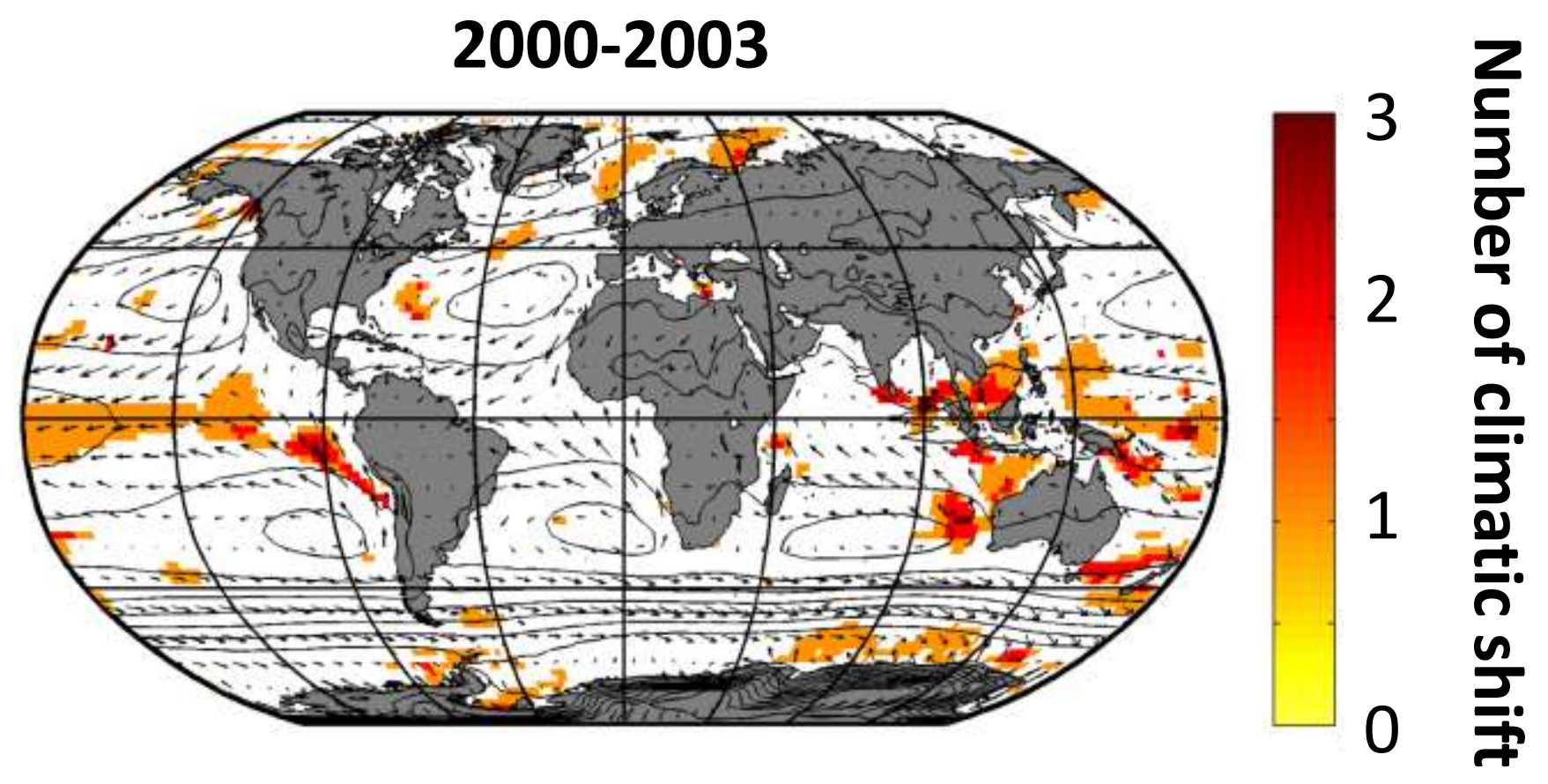

Black arrow: direction and strengh of mean annual wind (1960-2015) Red line: isobar based on annual SLP for the period 1960-2015 White area: no significant species' shift (threshold $>3$ )

Six climatic parameters were used: Annual sea level pressure, wind intensity, meridional and zonal wind, cloudiness, sea surface temperature. 


\section{Supplementary Figure 5}

\section{Number of climatic shifts in each geographical cell}

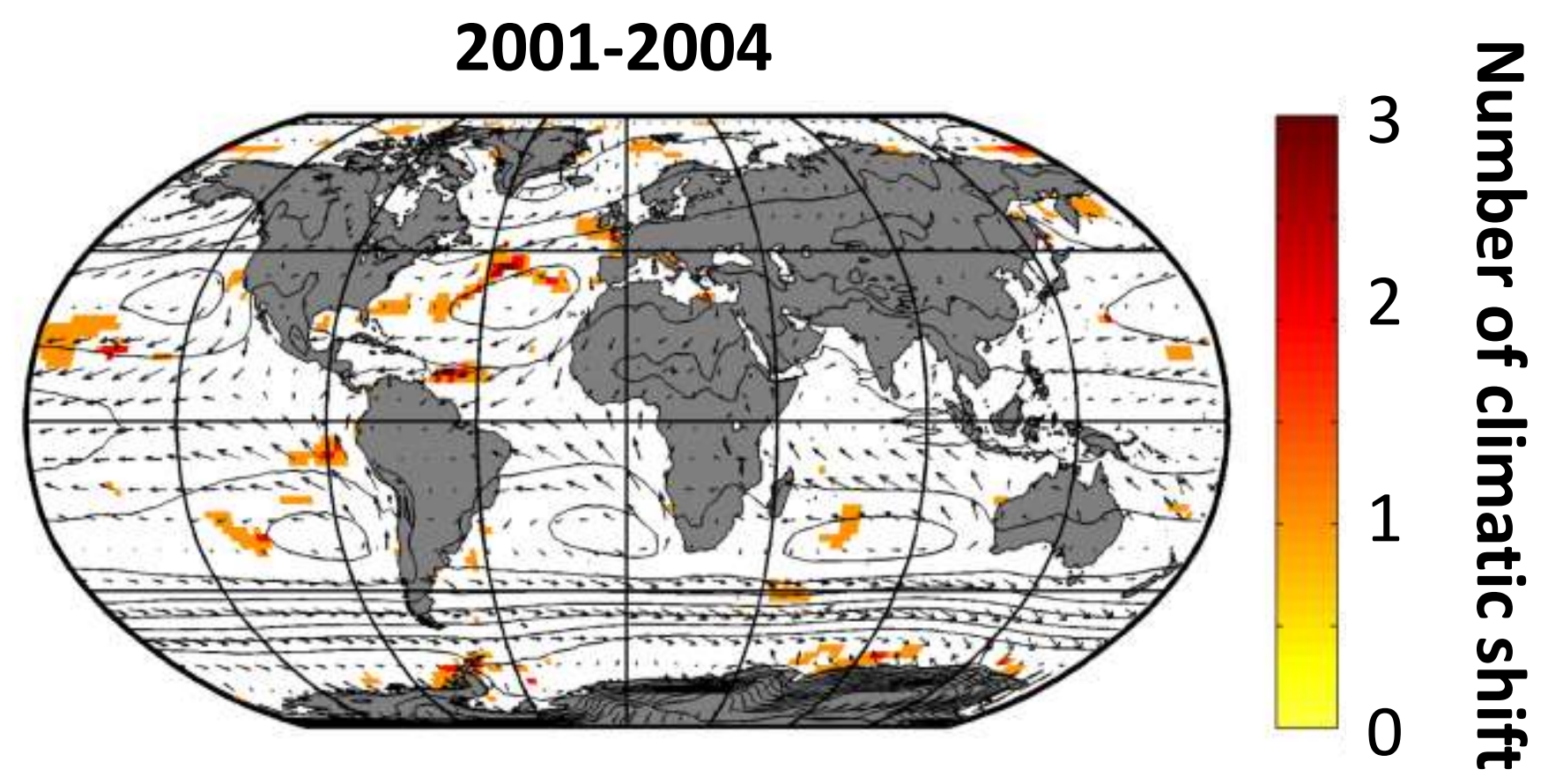

Black arrow: direction and strengh of mean annual wind (1960-2015) Red line: isobar based on annual SLP for the period 1960-2015 White area: no significant species' shift (threshold $>3$ )

Six climatic parameters were used: Annual sea level pressure, wind intensity, meridional and zonal wind, cloudiness, sea surface temperature. 


\section{Supplementary Figure 5}

\section{Number of climatic shifts in each geographical cell}

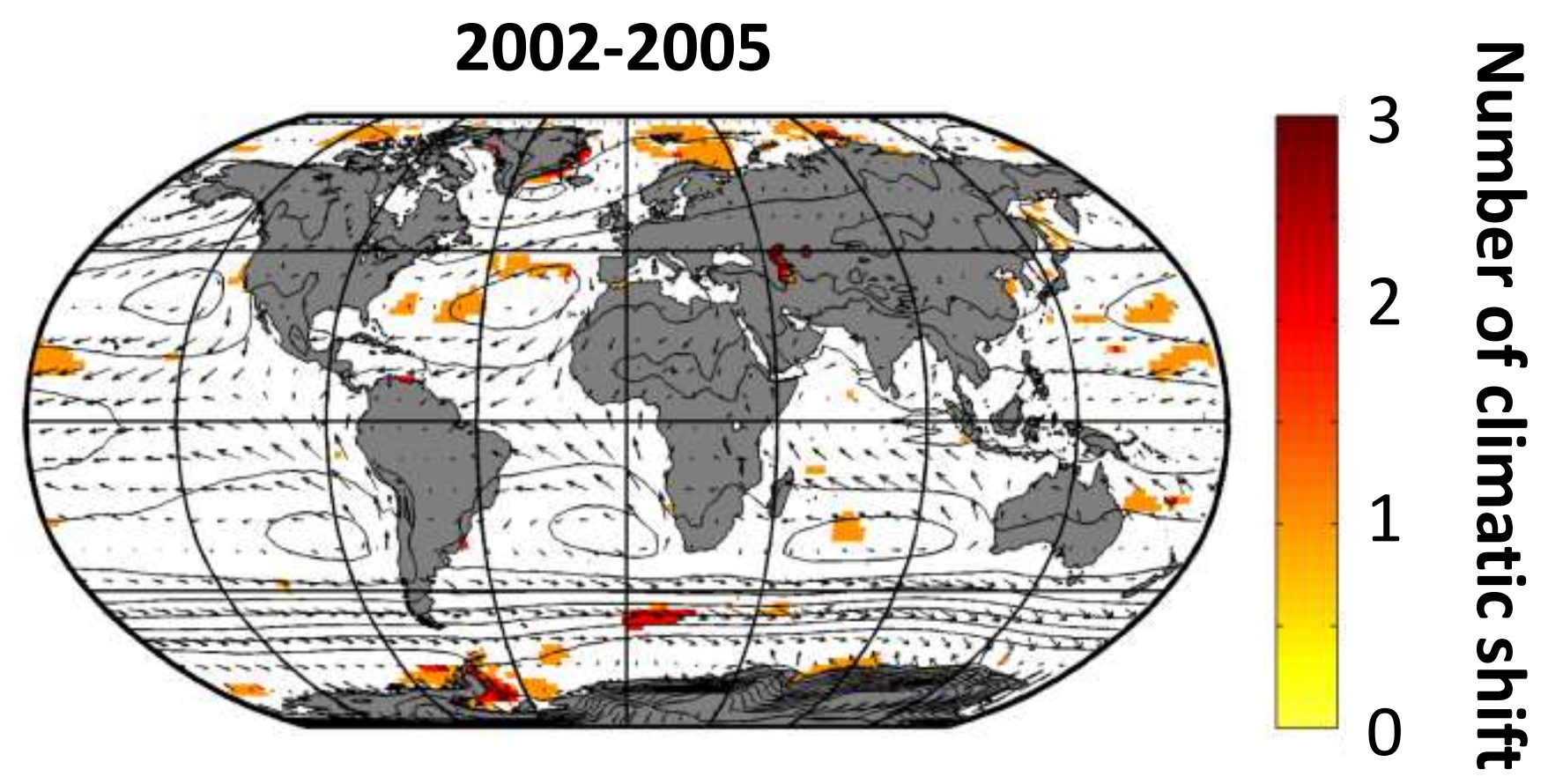

Black arrow: direction and strengh of mean annual wind (1960-2015) Red line: isobar based on annual SLP for the period 1960-2015 White area: no significant species' shift (threshold $>3$ )

Six climatic parameters were used: Annual sea level pressure, wind intensity, meridional and zonal wind, cloudiness, sea surface temperature. 


\section{Supplementary Figure 5}

\section{Number of climatic shifts in each geographical cell}

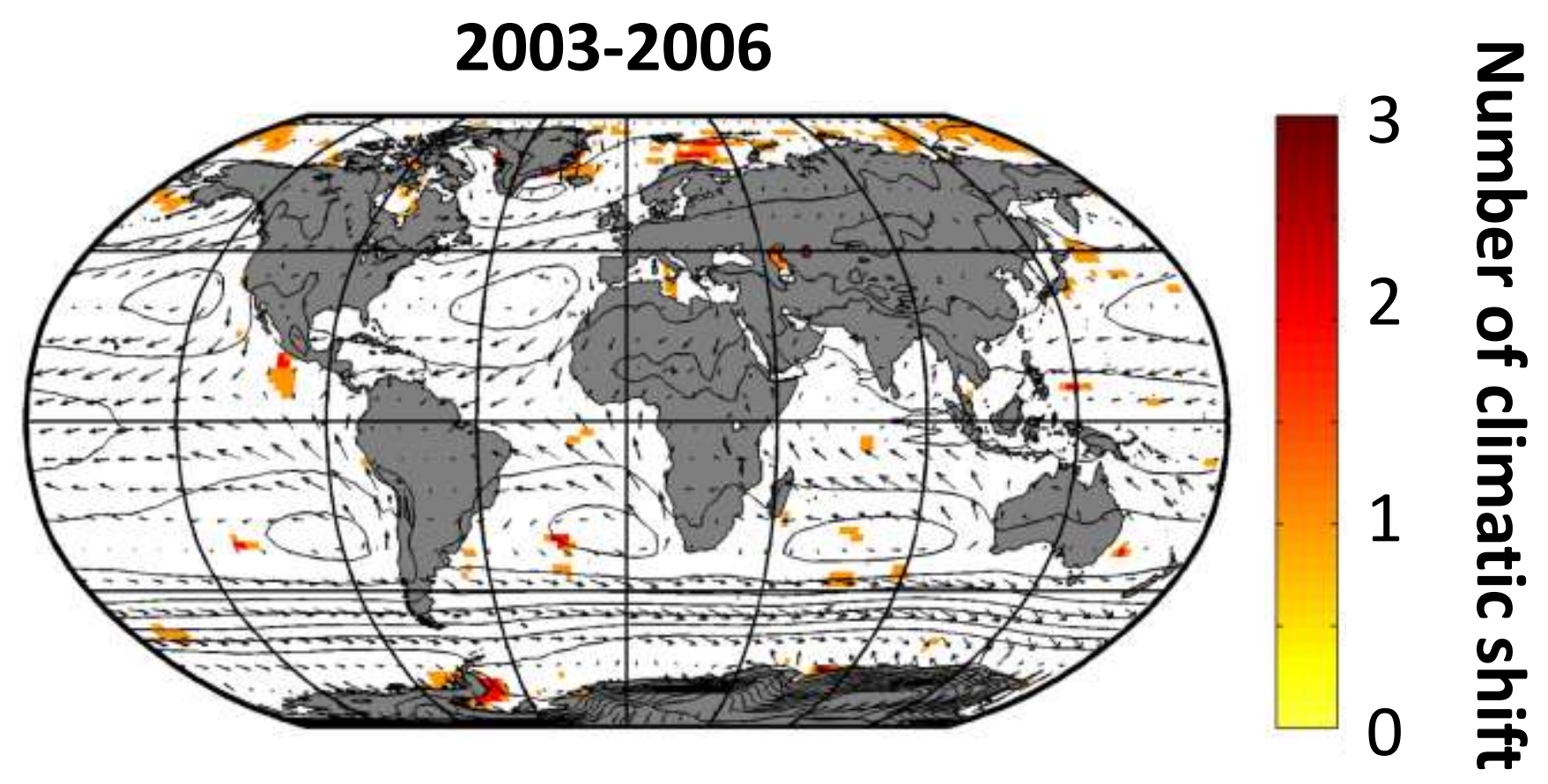

Black arrow: direction and strengh of mean annual wind (1960-2015) Red line: isobar based on annual SLP for the period 1960-2015 White area: no significant species' shift (threshold $>3$ )

Six climatic parameters were used: Annual sea level pressure, wind intensity, meridional and zonal wind, cloudiness, sea surface temperature. 


\section{Supplementary Figure 5}

\section{Number of climatic shifts in each geographical cell}

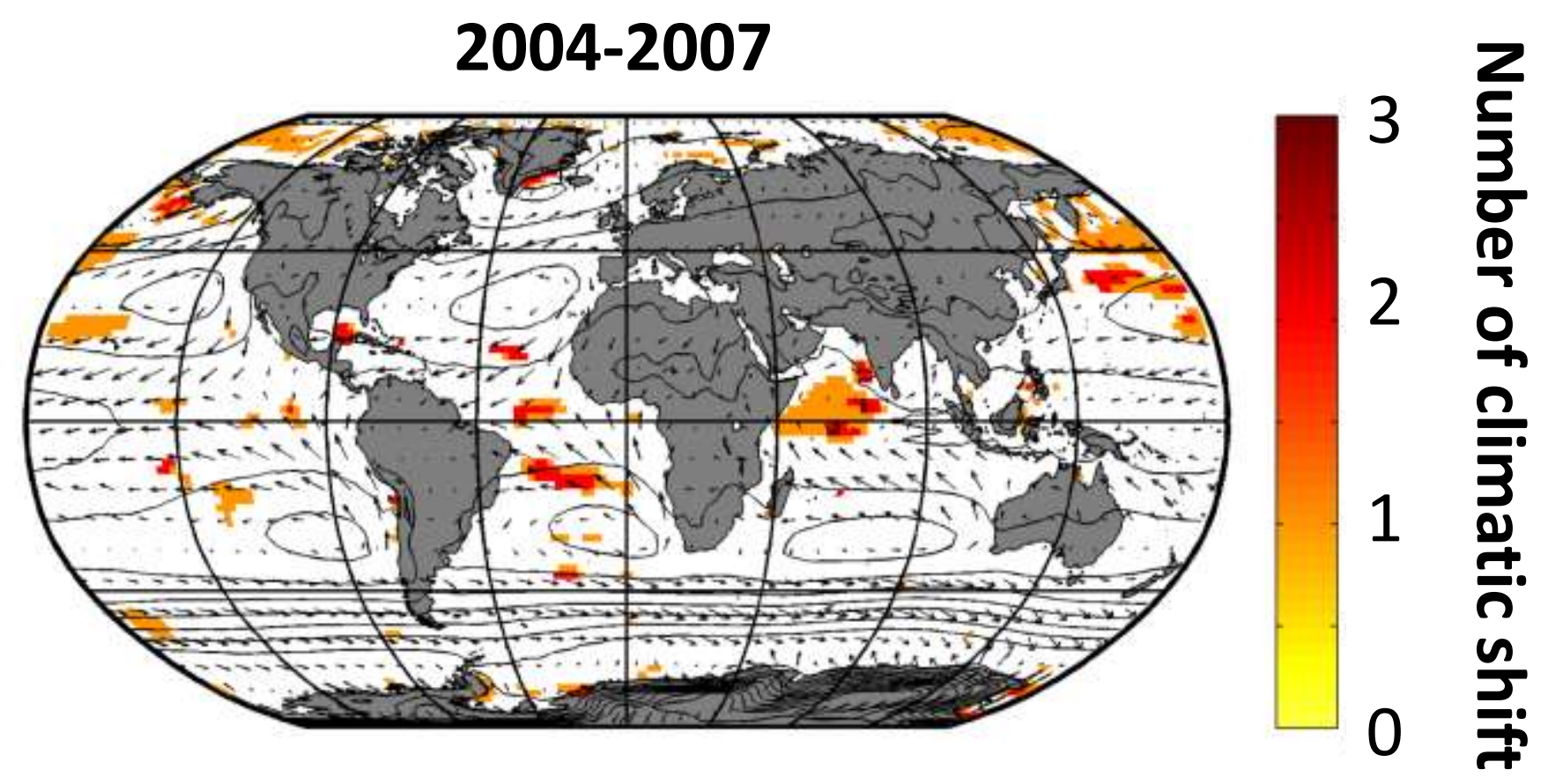

Black arrow: direction and strengh of mean annual wind (1960-2015) Red line: isobar based on annual SLP for the period 1960-2015 White area: no significant species' shift (threshold $>3$ )

Six climatic parameters were used: Annual sea level pressure, wind intensity, meridional and zonal wind, cloudiness, sea surface temperature. 


\section{Supplementary Figure 5}

\section{Number of climatic shifts in each geographical cell}

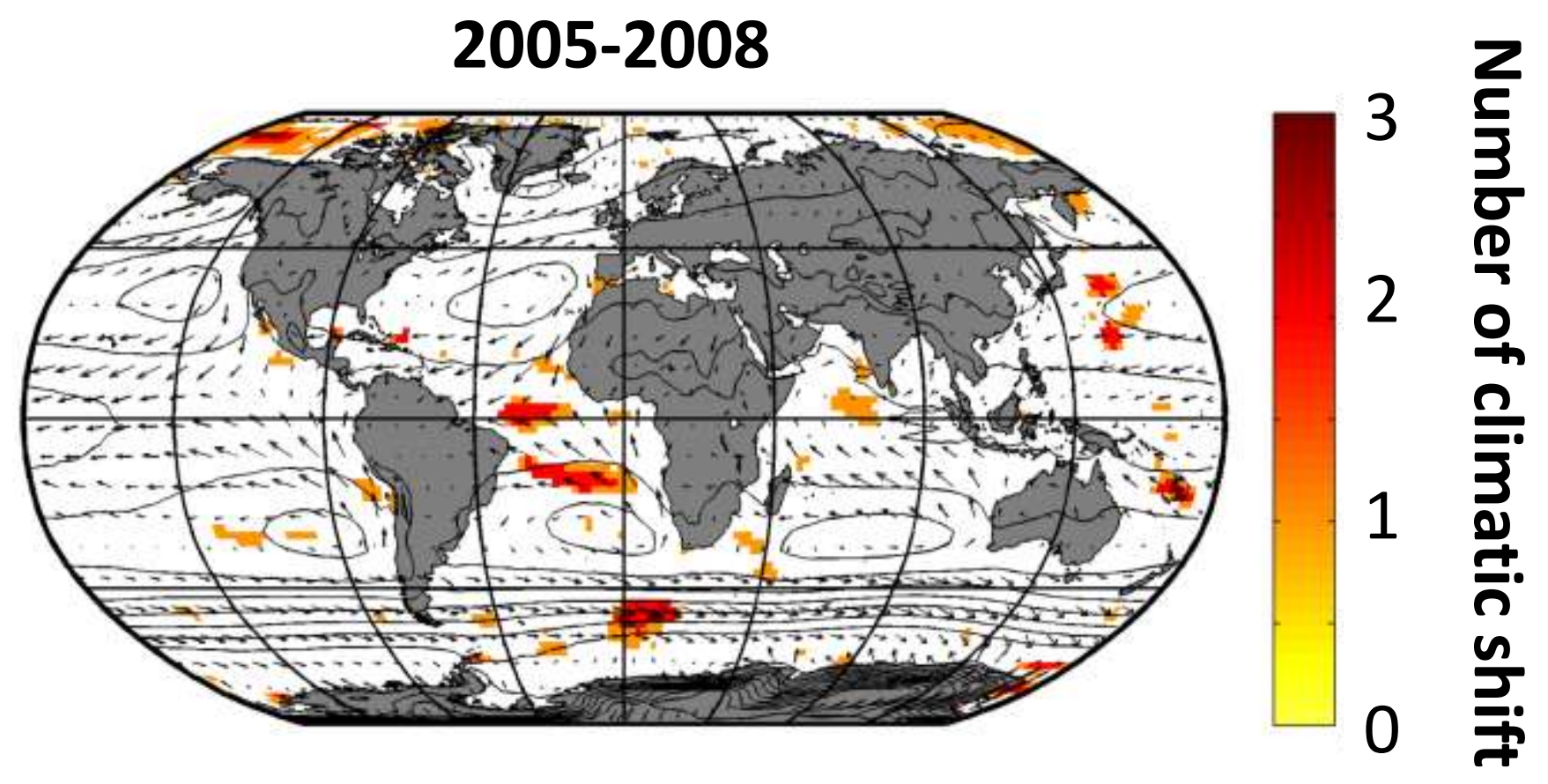

Black arrow: direction and strengh of mean annual wind (1960-2015) Red line: isobar based on annual SLP for the period 1960-2015 White area: no significant species' shift (threshold $>3$ )

Six climatic parameters were used: Annual sea level pressure, wind intensity, meridional and zonal wind, cloudiness, sea surface temperature. 


\section{Supplementary Figure 5}

\section{Number of climatic shifts in each geographical cell}

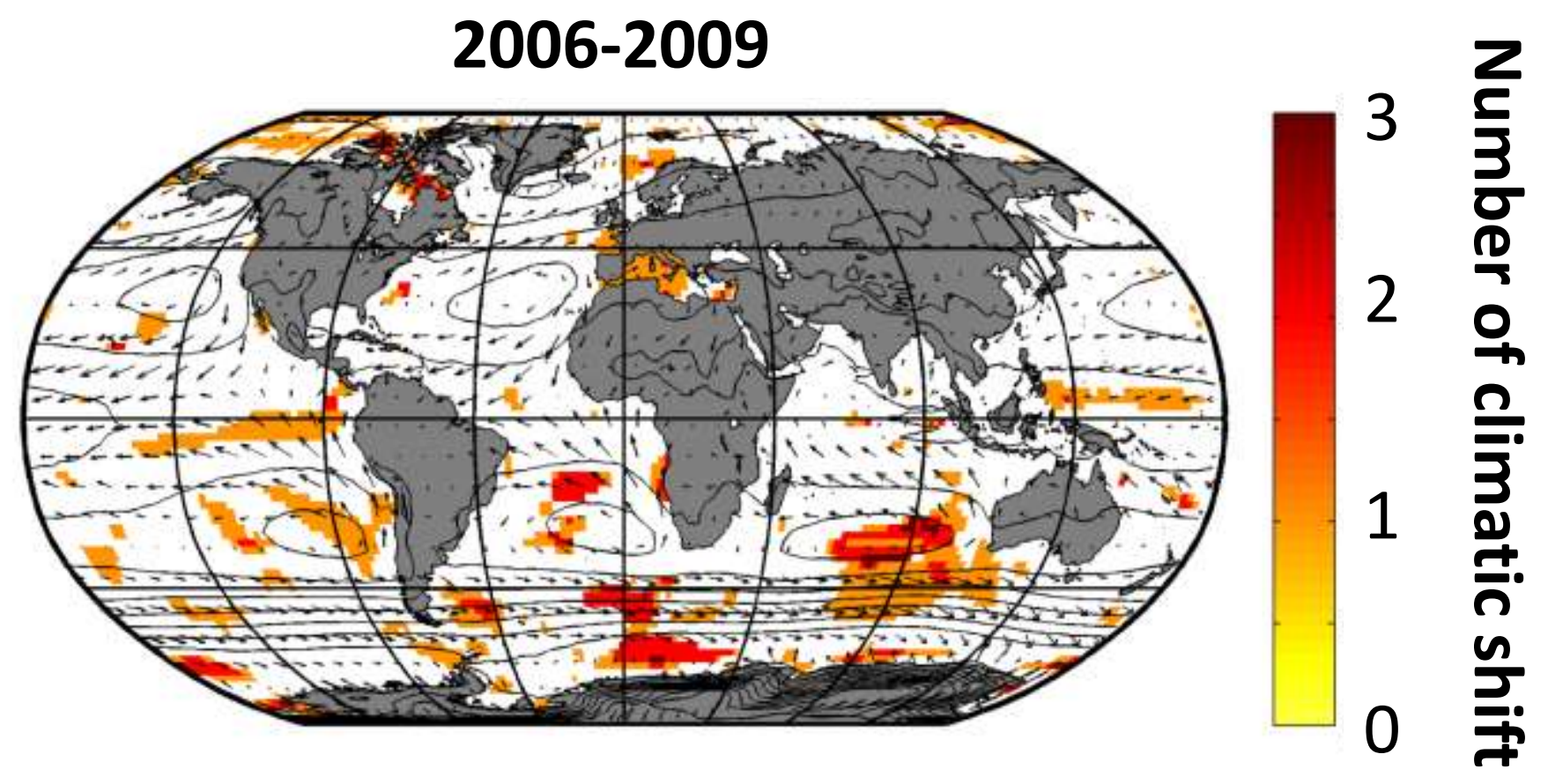

Black arrow: direction and strengh of mean annual wind (1960-2015) Red line: isobar based on annual SLP for the period 1960-2015 White area: no significant species' shift (threshold $>3$ )

Six climatic parameters were used: Annual sea level pressure, wind intensity, meridional and zonal wind, cloudiness, sea surface temperature. 


\section{Supplementary Figure 5}

\section{Number of climatic shifts in each geographical cell}

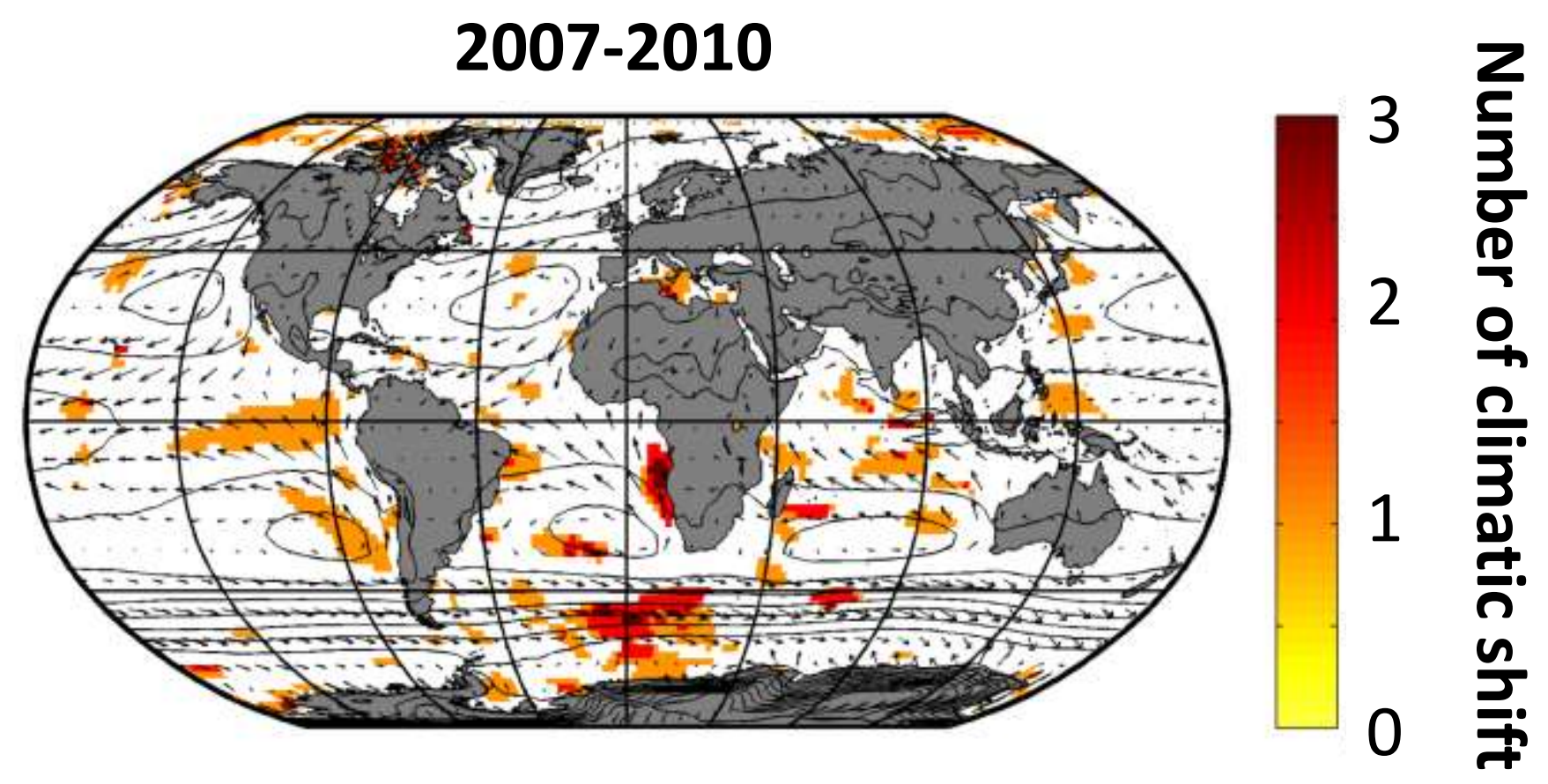

Black arrow: direction and strengh of mean annual wind (1960-2015) Red line: isobar based on annual SLP for the period 1960-2015 White area: no significant species' shift (threshold $>3$ )

Six climatic parameters were used: Annual sea level pressure, wind intensity, meridional and zonal wind, cloudiness, sea surface temperature. 


\section{Supplementary Figure 5}

\section{Number of climatic shifts in each geographical cell}

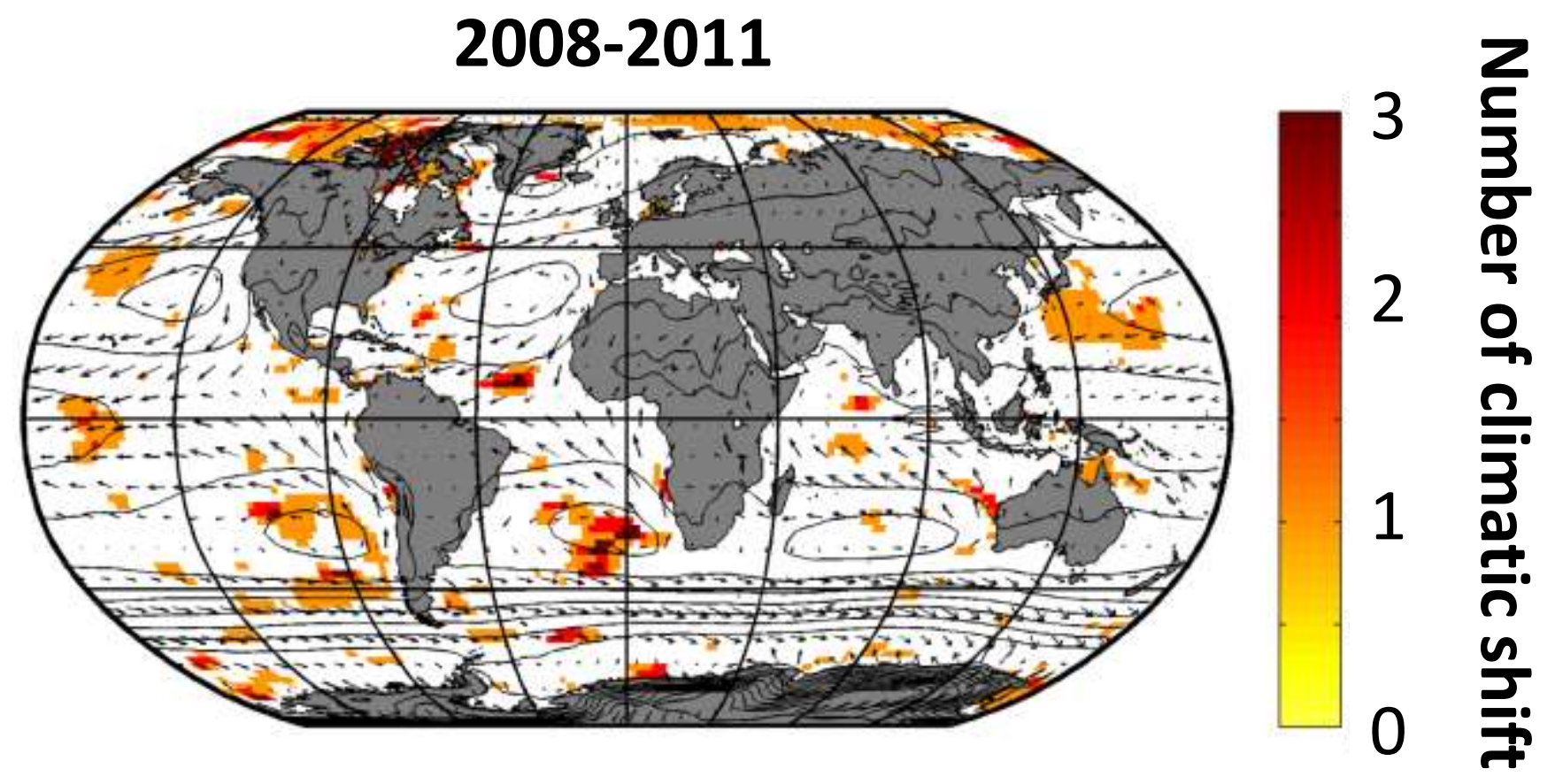

Black arrow: direction and strengh of mean annual wind (1960-2015) Red line: isobar based on annual SLP for the period 1960-2015 White area: no significant species' shift (threshold $>3$ )

Six climatic parameters were used: Annual sea level pressure, wind intensity, meridional and zonal wind, cloudiness, sea surface temperature. 


\section{Supplementary Figure 5}

\section{Number of climatic shifts in each geographical cell}

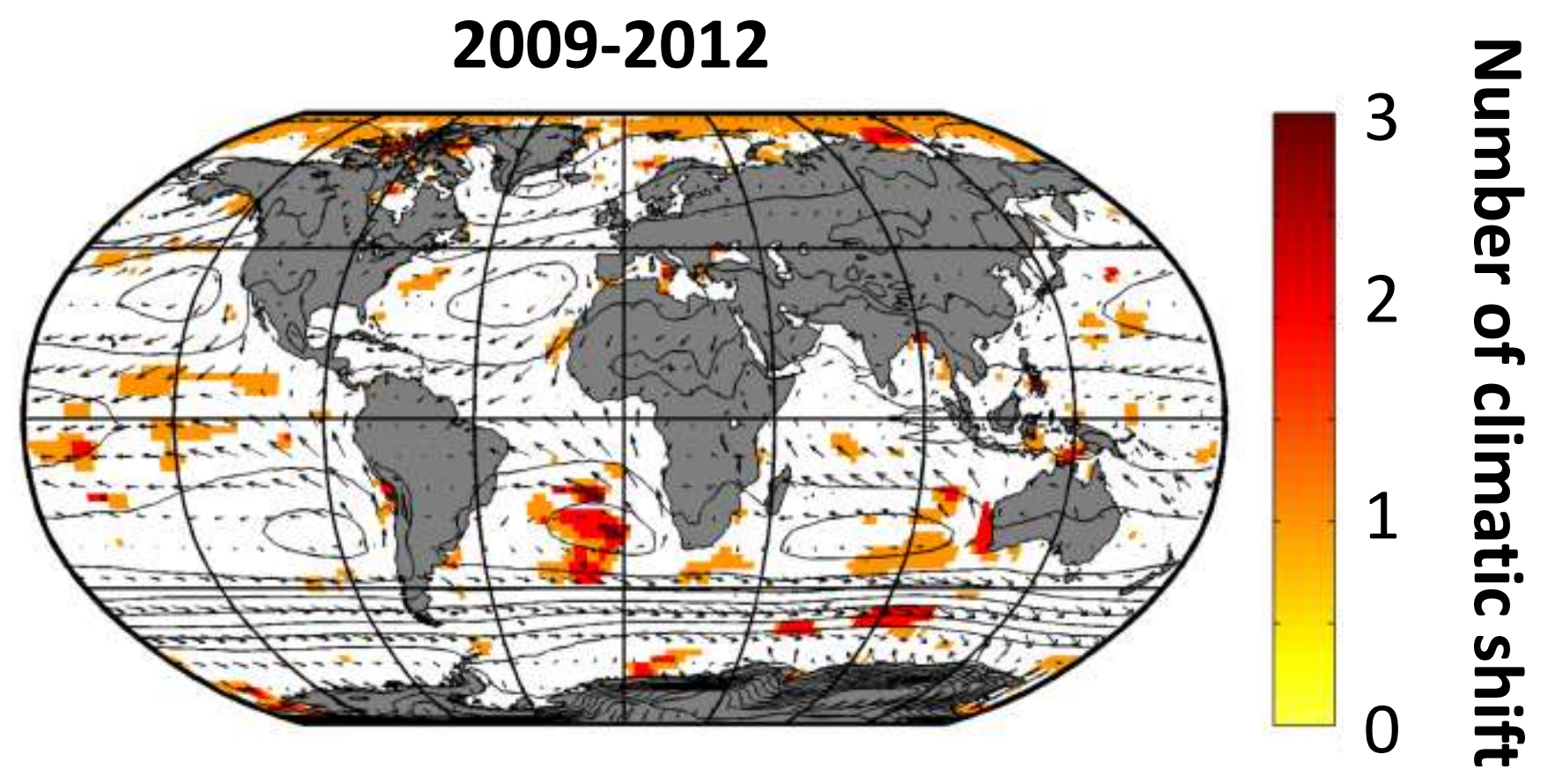

Black arrow: direction and strengh of mean annual wind (1960-2015) Red line: isobar based on annual SLP for the period 1960-2015 White area: no significant species' shift (threshold $>3$ )

Six climatic parameters were used: Annual sea level pressure, wind intensity, meridional and zonal wind, cloudiness, sea surface temperature. 


\section{Supplementary Figure 5}

\section{Number of climatic shifts in each geographical cell}

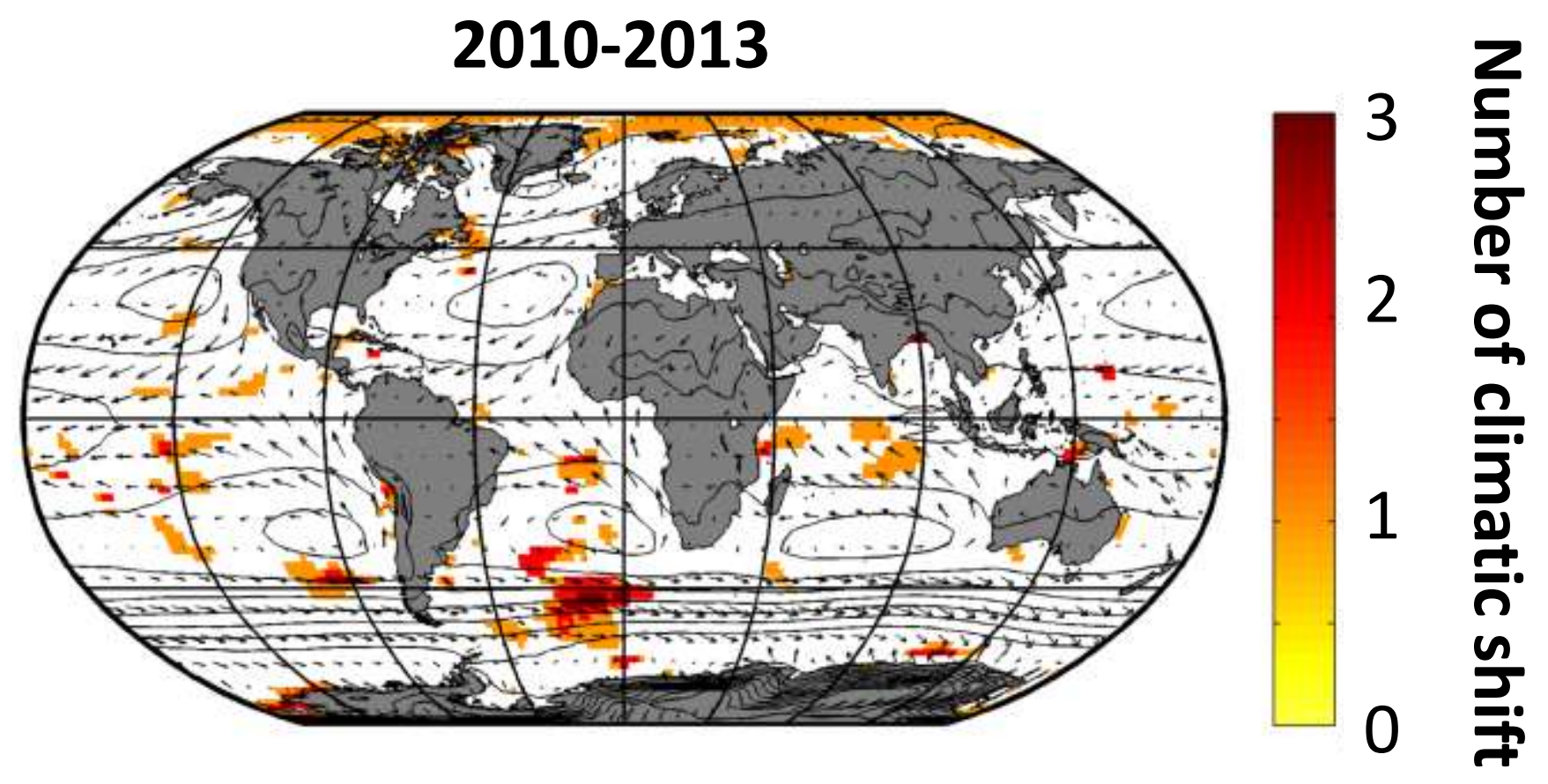

Black arrow: direction and strengh of mean annual wind (1960-2015) Red line: isobar based on annual SLP for the period 1960-2015 White area: no significant species' shift (threshold $>3$ )

Six climatic parameters were used: Annual sea level pressure, wind intensity, meridional and zonal wind, cloudiness, sea surface temperature. 


\section{Supplementary Figure 5}

\section{Number of climatic shifts in each geographical cell}

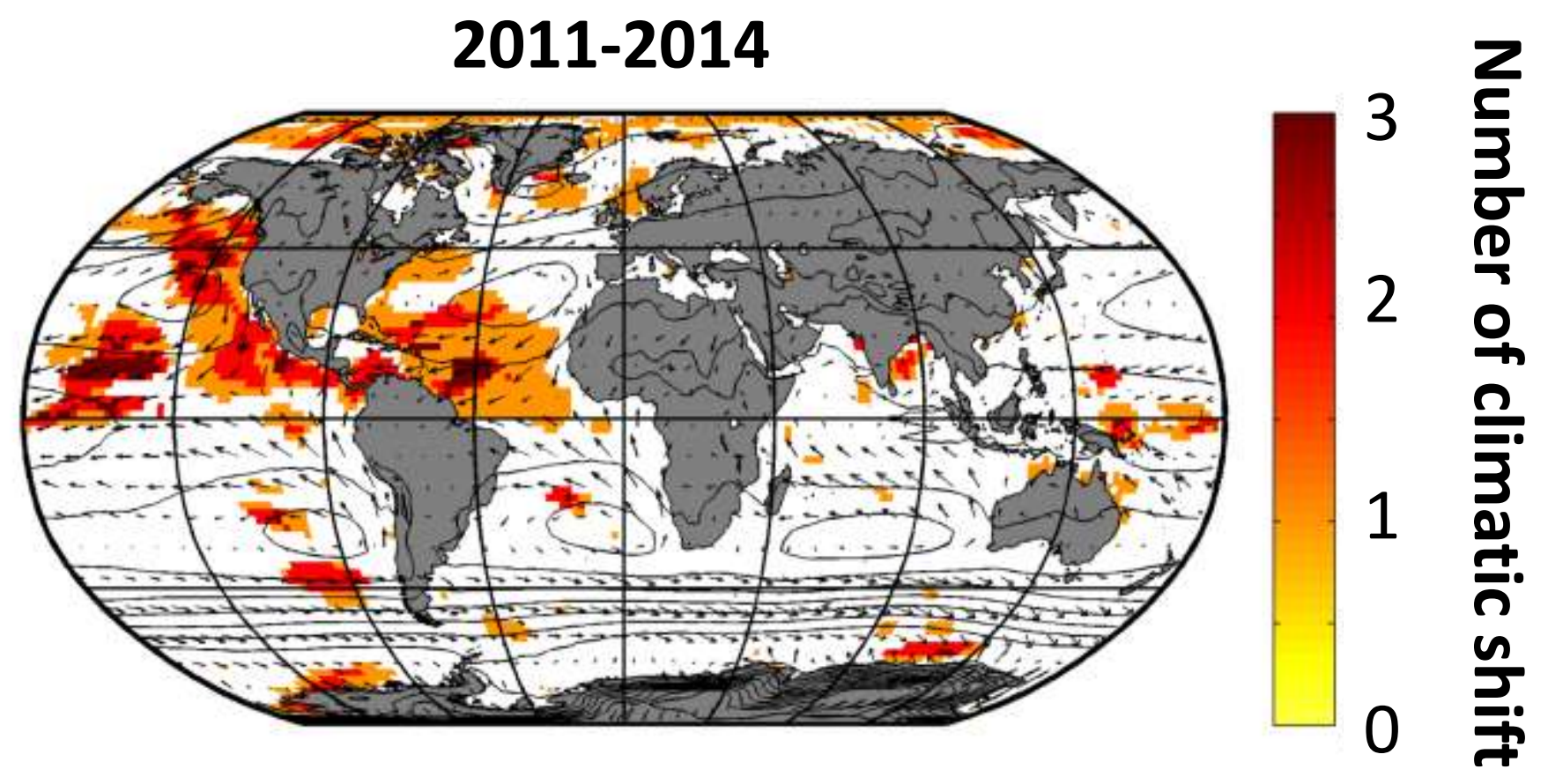

Black arrow: direction and strengh of mean annual wind (1960-2015) Red line: isobar based on annual SLP for the period 1960-2015 White area: no significant species' shift (threshold $>3$ )

Six climatic parameters were used: Annual sea level pressure, wind intensity, meridional and zonal wind, cloudiness, sea surface temperature. 


\section{Supplementary Figure 5}

\section{Number of climatic shifts in each geographical cell}

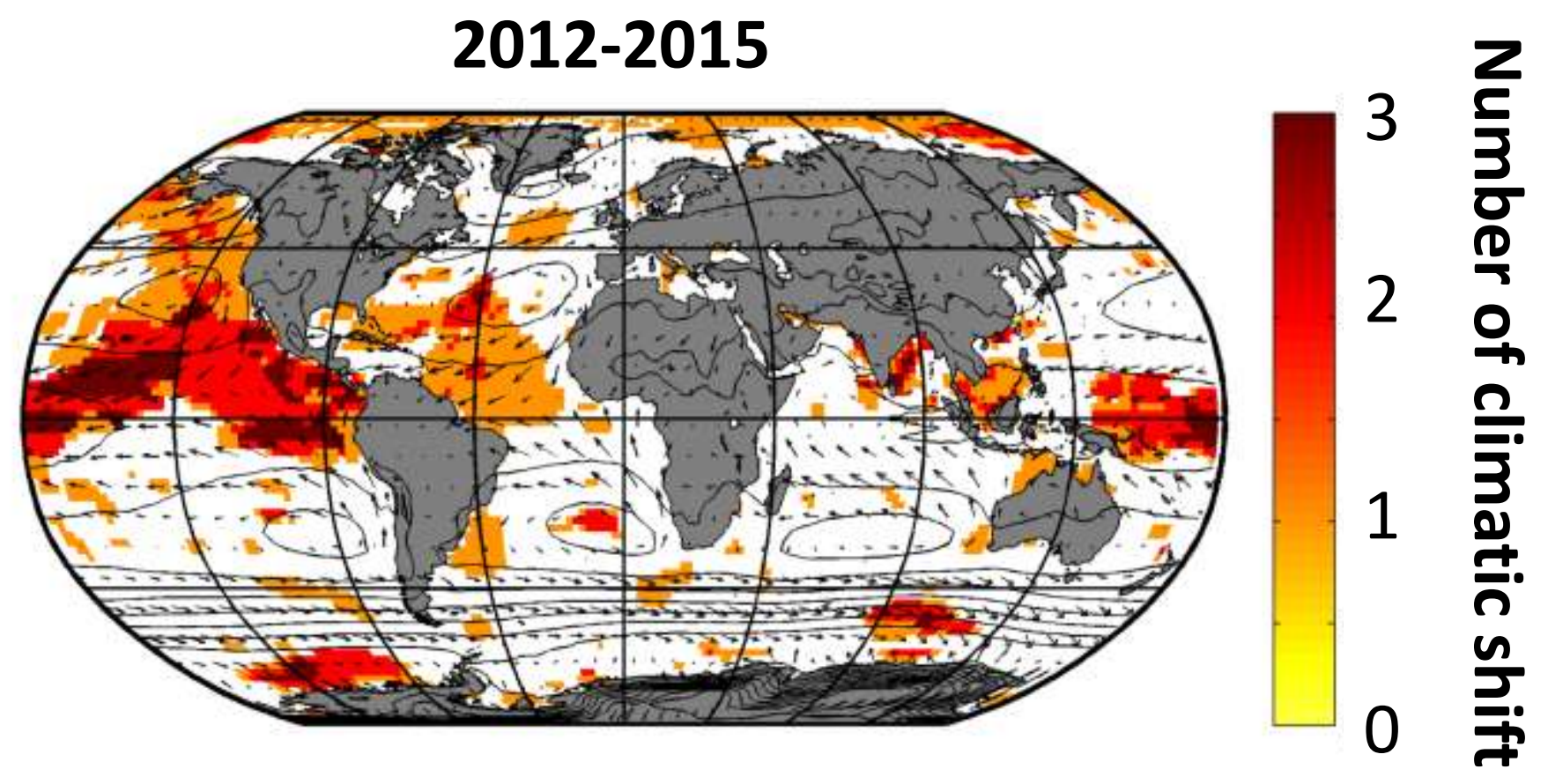

Black arrow: direction and strengh of mean annual wind (1960-2015) Red line: isobar based on annual SLP for the period 1960-2015 White area: no significant species' shift (threshold $>3$ )

Six climatic parameters were used: Annual sea level pressure, wind intensity, meridional and zonal wind, cloudiness, sea surface temperature. 